\title{
WestVirginiaUniversity
}

THE RESEARCH REPOSITORY @ WVU

Graduate Theses, Dissertations, and Problem Reports

2011

\section{Transport processes in low turbulence flows associated with enclosing hoods}

\author{
Ertan Karaismail \\ West Virginia University
}

Follow this and additional works at: https://researchrepository.wvu.edu/etd

\section{Recommended Citation}

Karaismail, Ertan, "Transport processes in low turbulence flows associated with enclosing hoods" (2011). Graduate Theses, Dissertations, and Problem Reports. 3035.

https://researchrepository.wvu.edu/etd/3035

This Dissertation is protected by copyright and/or related rights. It has been brought to you by the The Research Repository @ WVU with permission from the rights-holder(s). You are free to use this Dissertation in any way that is permitted by the copyright and related rights legislation that applies to your use. For other uses you must obtain permission from the rights-holder(s) directly, unless additional rights are indicated by a Creative Commons license in the record and/ or on the work itself. This Dissertation has been accepted for inclusion in WVU Graduate Theses, Dissertations, and Problem Reports collection by an authorized administrator of The Research Repository @ WVU.

For more information, please contact researchrepository@mail.wvu.edu. 
TRANSPORT PROCESSES IN LOW TURBULENCE FLOWS ASSOCIATED WITH ENCLOSING HOODS

\author{
ERTAN KARAISMAIL
}

\author{
DISSERTATION SUBMITTED TO THE \\ COLLEGE OF ENGINEERING AND MINERAL RESOURCES \\ AT WEST VIRGINIA UNIVERSITY \\ IN PARTIAL FULFILLMENT OF THE REQUIREMENTS \\ FOR THE DEGREE OF
}

\author{
DOCTOR OF PHILOSOPHY \\ IN \\ MECHANICAL ENGINEERING
}

Ismail Celik, Ph.D., Chair

Nigel N. Clark, Ph.D.

Steve E. Guffey, Ph.D.

Wade W. Huebsch, Ph.D.

John M. Kuhlman, Ph.D.

MECHANICAL AND AEROSPACE ENGINEERING DEPARTMENT

Morgantown, West Virginia

2011

Keywords: Computational Fluid Dynamics, Immersed Boundary Method, Domain Decomposition Method, Bench-top enclosing hoods, Worker exposure assessment 


\title{
ABSTRACT
}

\section{TRANSPORT PROCESSES IN LOW TURBULENCE FLOWS ASSOCIATED WITH ENCLOSING HOODS}

\author{
Ertan Karaismail
}

Effective exhaust hoods are critical for protection of workers from airborne contaminants. The present study investigates the flow dynamics and associated contaminant dispersion in the near-wake of a worker working at a benchtop enclosing hood. The focus is primarily placed on evaluating the effects of different factors such as cross-draft, body heat and body shape on the dynamics of the wake flow and eventually on the exposure level. For this purpose, extensive two- and three-dimensional Unsteady Reynolds-Averaged Navier-Stokes (URANS) simulations are carried out using the commercial Computational Fluid Dynamics (CFD) software, called FLUENT, with various turbulence models, such as SST $k-\omega, \mathrm{RNG} k-\epsilon$ and Standard $k-\epsilon$. The predictions from two-dimensional cases suggest that the SST $k-\omega$ model is more responsive to unsteady flow dynamics. The RNG $k-\epsilon$ and Standard $k-\epsilon$ models, on the other hand, are found overly diffusive, and hence, are not as successful as SST $k-\omega$ model in capturing the unsteady phenomena.

Three-dimensional simulations indicate that the flow separation around the worker's body is, to a large extent, hindered by the acceleration of the flow under the effect of suction. Hence, the anticipated lateral recirculation zones expanding into the hood do not always form. However, dynamic large-scale helical motions are found to characterize the flow from the hood face to the back of the hood. 
The simulated flow patterns are compared with the observations from concurrent smoke visualization experiments and they seem to capture the observed average flow field well. The negative effect of body heat on the exposure level is more pronounced at low flow rates. Comparison of predictions using simple, round, and complex, anthropometrically-scaled manikins reveals that the simple, round body is an acceptable representation of the realistic body from the viewpoint of exposure level. The predicted exposure trends agree well with the experimental measurements. However, the quantitative values of the predicted concentrations are highly sensitive to the grid resolution.

In a further attempt to apply Large Eddy Simulation (LES) to such a dynamic problem, an in-house CFD code, called DREAM, has been made parallel using Domain Decomposition Technique and the predictions are validated against benchmark solutions available in the literature. Moreover, a strongly monotone Quasi-Second Order Upwind (QSOU) convection scheme is implemented for accurate solution of scalar transport along with a feedback forcing based Immersed Boundary (IB) method to account for solid bodies immersed in the fluid. The new code (DREAM_P) is applied for LES of the original worker-hood problem. The results agree favorably with the URANS simulations. The DREAM_P forms a much simpler computational platform compared to FLUENT for further study of flows dominated by bluff bodies. 
to my beloved family and to all those who appreciate science 


\section{ACKNOWLEDGMENTS}

I would like to gratefully thank to my advisor, Dr. Ismail Celik for his invaluable guidance and support throughout this study. His great mentorship provided me with the foundation that I need to be successful in this field. Also having me involved in various challenging projects provided me with the great opportunity to gain a broader breadth of experience.

I wish to express my gratitude to my committee members, Dr. Nigel Clark, Dr. Steve Guffey, Dr. Wade Huebsch and Dr. John Kuhlman for generously allotting their time and encouragement. In particular, I would like to thank to Dr. Steve Guffey for providing me with critical feedback and valuable resources. I have benefited from them a lot.

I would like to thank to the members of my research group who made these tough years less daunting and even enjoyable for me. I particularly would like to show my appreciation to Dr. Raju Pakalapati who has worked with me many times without any hesitation. I consider myself very lucky to witness his 'beautiful mind'. I should also thank to my buddy, John Redrow for his good company. We had so many good times that I will never forget.

I should offer my regards to my former advisor, Dr. Nevin Selcuk at METU who instilled dedication and discipline in me. Those were the key elements in accomplishing this dissertation process.

I also would like to acknowledge the National Institute of Occupational Safety and Health (NIOSH) for the financial support on this research (1 R01 OH008165 Enclosing Hood Effectiveness).

Most importantly, I would like to thank to my parents Nejla and Sayit Karaismail, and to my sister Ebru Karaismail for their help and faith in me. This 
dissertation would have never been possible without their unwavering support. I will be forever indebted to them for everything they have done for me.

Behind everything was my wonderful wife, Nihan who has to put up with me everyday. I have been feeling extremely fortunate for having her in my life and literally walking this road together. I just can not thank her enough for her love that manifests itself through unyielding devotion, understanding and tolerance.

On January 14th, 2010, our son, Daghan arrived and brought in happiness and fun to our life. Since then he is the sunshine to us. Thanks God for giving us such a wonderful boy. What a privilege!

Finally, I am deeply thankful to my in-laws, Asuman and Omer F. Cayan and again to my parents for taking their time out to watch our little one. Without their support this effort would not have been a success. 


\section{TABLE OF CONTENTS}

ABSTRACT .............................. ii

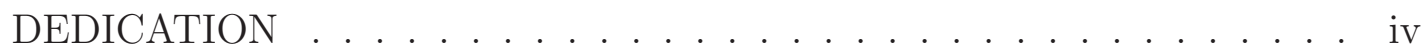

ACKNOWLEDGMENTS ..................... . . . .

TABLE OF CONTENTS ...................... vii

LIST OF TABLES . . . . . . . . . . . . . . . . . . . $\mathrm{x}$

LIST OF FIGURES . . . . . . . . . . . . . . . . . . . . xi

LIST OF SYMBOLS . . . . . . . . . . . . . . . . xvi CHAPTER

1 Introduction . . . . . . . . . . . . . . . . . 1

1.1 Flows Associated with Bench-top Enclosing Hoods . . . . . . 2

1.2 Turbulence Modeling . . . . . . . . . . . . . . . . . . 5

1.3 Numerical Issues . . . . . . . . . . . . . . . . . . . . . . . . 6

1.4 Literature Review . . . . . . . . . . . . . . . . . . . 8

1.5 Motivation and Objectives of the Study . . . . . . . . . . . 14

2 Governing Equations and Turbulence Models . . . . . . . . . . . . . 16

2.1 RANS Turbulence Models . . . . . . . . . . . . . . . 18

2.1.1 $k-\epsilon$ Group Models . . . . . . . . . . . . . . . . . . . 19

2.1.1.1 Standard $k-\epsilon$ Model . . . . . . . . . . . 19

2.1.1.2 The Renormalization Group (RNG) $k-\epsilon$ Model 21

2.1.2 Shear-Stress Transport (SST) $k-\omega$ Model . . . . . . . 22

2.2 Large Eddy Simulation . . . . . . . . . . . . . . . . . . 25

3 RANS Turbulence Model Performance . . . . . . . . . . . . . . . . 29

3.1 Two-dimensional Test Case . . . . . . . . . . . . . . . . . . . 29

4 Experiments and Numerical Simulations . . . . . . . . . . . . 41 
4.1 Experimental Setup . . . . . . . . . . . . . . . . . 41

4.2 Numerical Simulations . . . . . . . . . . . . . . . . . 43

4.2 .1 Isothermal Flow . . . . . . . . . . . . . . . . . . 47

4.2 .2 Flow unsteadiness . . . . . . . . . . . . . . . . . 61

4.2 .3 Effect of Direction of Draft . . . . . . . . . . . . 68

4.2 .4 Non-isothermal Flow . . . . . . . . . . . . . . . . . 69

4.2.5 Effect of Body Shape . . . . . . . . . . . . . . 77

5 Parallel Implementation of the DREAM _ . . . . . . . . . . 81

5.1 Parallel Algorithm and Code Structure . . . . . . . . . . . 81

5.2 Verification of Parallel Algorithm . . . . . . . . . . . . 86

5.2.1 Test Case I: Developing Flow between Parallel Plates 86

5.2.2 Test Case II: Lid-Driven Cavity Flow . . . . . . . . . . 89

5.2.3 Test Case III: Flow Over a Square Cylinder . . . . . . 95

6 Implementation of Quasi-Second Order Upwind Scheme into the DREAM . . . . . . . . . . . . . . . . . . . 101

6.1 Introduction to QSOU Scheme . . . . . . . . . . . 101

6.2 Formulation and Implementation . . . . . . . . . . 105

6.3 Verification of the Scalar Solver . . . . . . . . . . . . 107

6.3 .1 Test Case I: Diagonal Flow . . . . . . . . . . . . . 108

6.3 .2 Test Case II: Rotational Flow . . . . . . . . . . . . 109

7 Implementation of Immersed Boundary Method into the DREAM . 113

7.1 Feedback Momentum Forcing . . . . . . . . . . . . 117

7.2 Interpolation Scheme . . . . . . . . . . . . . . 120

7.3 Test Case: Uniform Flow over a Stationary Cylinder _. . . . 123

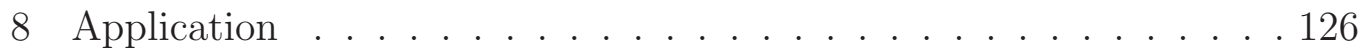

9 Conclusions and Future Recommendations . . . . . . . . . . 132

9.1 Conclusions . . . . . . . . . . . . . . . . . . . . . 132

9.2 Future Recommendations . . . . . . . . . . . . . . 134

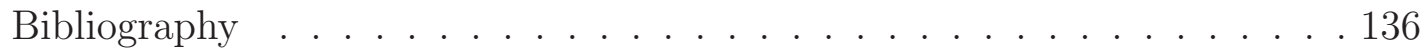




\section{APPENDICES}

A CAD Modeling of Anthropometrically-scaled Complex Manikin . 149 


\section{LIST OF TABLES}

1.1 Summary of the literature review on studies related to enclosing hoods 11

3.1 Number of Cells Used . . . . . . . . . . . . . . . . . . 32

3.2 Laminar and turbulent viscosities in the near-wall region $(x=$ $2.146 a, y=6.427 a) \ldots . \ldots . \ldots . . \ldots 34$

4.1 Air velocities used in the simulations . . . . . . . . . . . . . 45

4.2 Calculated dimensionless quantities for various $V_{\text {Cross-draft }}$ to $V_{\text {Face }}$

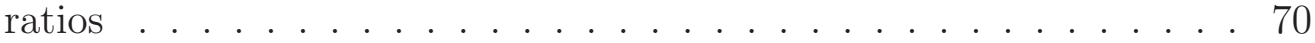

6.1 Maximum values of the scalar . . . . . . . . . . . . . 111 


\section{LIST OF FIGURES}

3.1 Schematic representation of the physical system . . . . . . . . . . . 30

3.2 Detail view of grid (G6) around the ellipse . . . . . . . . . . . . . 32

3.3 Variation of a) angle of separation, b) nondimensional recirculation zone length $(a=0.6 \mathrm{~m})$, with $\Delta y_{1} \ldots \ldots \ldots$. . . . . . . 34

3.4 Streamtraces predicted on coarse grid (G1) with the standard $k-\epsilon$

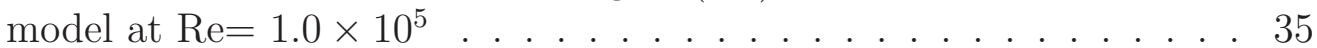

3.5 Comparison of the streamtraces predicted by fully resolved (G7) SST $k-\omega$ and RNG $k-\epsilon$ models at $\mathrm{Re}=1.0 \times 10^{5} \ldots . . . . . .36$

3.6 Predicted streamtraces at 4000 time step at $\mathrm{Re}=1.0 \times 10^{4}$, a) $\mathrm{SST}$ $k-\omega$ model, b) RNG $k-\epsilon$ model, c) standard $k-\epsilon$ model . . . . . 37

3.7 Predicted turbulent viscosities at 4000 time step at $\operatorname{Re}=1.0 \times 10^{4}$, a) SST $k-\omega$ model, b) RNG $k-\epsilon$ model, c) standard $k-\epsilon$ model . 37

3.8 Streamwise velocity from SST $k-\omega$ model at a point $(x=2.4 a, y=$ $6.57 a$ ) at $\mathrm{Re}=1.0 \times 10^{4}$, a) time history, b) power spectral density

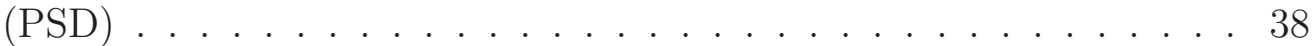

3.9 Time history of streamwise velocity and corresponding PSD spectrum at a point $(x=2.4 a, y=6.57 a)$ at $\operatorname{Re}=1.0 \times 10^{3}$, a-b) Laminar, c-d) SST $k-\omega$ model . . . . . . . . . . . . . . . . 39

4.1 Schematic representation of the wind tunnel and its contents. Blue arrows indicate direction of cross-draft. (Courtesy of Dr. S.Guffey) . 42

4.2 Manikins used in the experiments a) anthropometrically scaled, b) simple . . . . . . . . . . . . . . . . . . 43

4.3 Detail view of coarse mesh . . . . . . . . . . . . . . . . . . 45

4.4 Location of data monitoring/sampling points (Mouth; Point 1: $x=2.03 \mathrm{~m}, y=1.45 \mathrm{~m}, z=1.83 \mathrm{~m}$, Nose; Point $2: x=2.09 \mathrm{~m}, y=1.42 \mathrm{~m}$, $z=1.84 \mathrm{~m}$, Point 3: $x=2.09 \mathrm{~m}, y=1.42 \mathrm{~m}, z=1.84 \mathrm{~m}$ ) a) simple manikin, b) complex manikin . . . . . . . . . . . . . . . . . 47

4.5 Grid sensitivity of the long-time-averaged concentrations for the simple manikin a) Point 1, b) Point 2 . . . . . . . . . . . . . 49

4.6 Flow and concentration fields at the hood face for Case D a) Coarse grid b) Fine grid . . . . . . . . . . . . . . . . . 50 50 
4.7 Planes on which results are presented; $x y$-plane-1: $z=1.8 \mathrm{~m}$, $x y$-plane-2: $\quad z=1.7 \mathrm{~m}, \quad y z$-plane- $1: \quad x=2.066 \mathrm{~m}, \quad y z-$ plane- 2 : $x=2.046 \mathrm{~m}, x z$-plane: $y=1.3 \mathrm{~m} \ldots \ldots \ldots \ldots$

4.8 Flow and concentration fields at the hood face a) Case D, b) Case A, c) Case G, d) Case F . . . . . . . . . . . . . . . . . 51

4.9 Three-dimensional stream-traces . . . . . . . . . . . . . . . . . 52

4.10 Snapshot showing the vortices in front of the worker (Case D) . . . 52

4.11 Flow and concentration fields for Case $\mathrm{G}$ at $z=1.7 \mathrm{~m} \ldots \ldots$. . . 54

4.12 Snapshot showing the recirculation zone attached to the left wall of the hood (Case A, top view) . . . . . . . . . . . 54

4.13 Trace of smoke streamlined towards the back of the hood (Case D) . 55

4.14 Airflow flow patterns and concentration fields on the $x z$-plane a) Case D, b) Case A, c) Case G, d) Case F . . . . . . . . . . . 56

4.15 Comparison of experimentally observed and numerically predicted flow fields around the worker's head (Case D) a) Experimental b) Numerical . . . . . . . . . . . . . . . . . . . . . . . . 57

4.16 Airflow flow patterns and concentration fields at the hood face a) Case D, b) Case A, c) Case G, d) Case F . . . . . . . . . . 58

4.17 Vortical motion above the work surface (Case A) . . . . . . . 58

4.18 Concentration distributions on the surface of the worker (manikin) a) Case D, b) Case A, c) Case G, d) Case F . . . . . . . . . . 59

4.19 Flow and turbulent kinetic energy fields at the hood face a) Case D, b) Case A, c) Case G, d) Case F . . . . . . . . . . . . . 60

4.20 Mixing in the breathing zone of the manikin (Case D) $\ldots \ldots \ldots 6$

4.21 Vorticity contours and stream-traces at the hood face for Case $\mathrm{G}$ a)

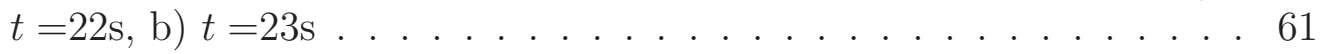

4.22 Eddies tumbling on a vertical plane on the right of manikin $(y z$-plane-2, Case G) a) $t=65 \mathrm{~s}$, b) $t=67 \mathrm{~s} \ldots \ldots \ldots 2$

4.23 Time history of $z$-velocity and concentration along with their PSD spectrum at Point 1 for Case D a) $z$-velocity b) concentration . . . 64

4.24 Time history of $z$-velocity and concentration along with their PSD spectrum at Point 2 for Case D a) $z$-velocity b) concentration . . . 64

4.25 Time history of $z$-velocity and concentration along with their PSD spectrum at Point 1 for Case A a) $z$-velocity b) concentration . . . 65

4.26 Time history of $z$-velocity and concentration along with their PSD spectrum at Point 2 for Case A a) $z$-velocity b) concentration . . . 65

4.27 Time history of $z$-velocity and concentration along with their PSD spectrum at Point 1 for Case G a) $z$-velocity b) concentration . . . 66 
4.28 Time history of $z$-velocity and concentration along with their PSD spectrum at Point 2 for Case G a) $z$-velocity b) concentration . . . 66

4.29 Time history of $z$-velocity and concentration along with their PSD spectrum at Point 1 for Case $\mathrm{F}$ a) $z$-velocity b) concentration . . . . 67

4.30 Time history of $z$-velocity and concentration along with their PSD spectrum at Point 2 for Case F a) $z$-velocity b) concentration . . . . 67

4.31 Time history of $z$-velocity and concentration at Point 3 for Case D a) $z$-velocity b) concentration . . . . . . . . . . . 68

4.32 Average flow field in the wake of the manikin for Case $\mathrm{G}$ a) waist level b) shoulder level . . . . . . . . . . . . . . . . . . . 69

4.33 Comparison of long-time-averaged concentrations from simulations with and without buoyancy a) Point 1, b) Point 2 . . . . . . . . 71

4.34 Comparison of temperature fields for a) Case D and b) Case G . . . 73

4.35 Comparison of turbulent kinetic energy fields at the hood face for Case D a) without buoyancy, b) with buoyancy . . . . . . . . . . . 73

4.36 Time history of $z$-velocity at Point 2 a) Case D without buoyancy, b) Case D with buoyancy, c) Case F without buoyancy, d) Case F with buoyancy . . . . . . . . . . . . . . . 74

4.37 Time history of concentrations at Point 2 a) Case D without buoyancy, b) Case D with buoyancy, c) Case F without buoyancy, d) Case F with buoyancy . . . . . . . . . . . . . . . . 74

4.38 Instantaneous concentrations at Point 1 under buoyancy effects (Case G), a) with RNG $k-\epsilon$ turbulence model, b) Without a turbulence model . . . . . . . . . . . . . . . . . . . . . . 75

4.39 Instantaneous concentrations at Point 1 under buoyancy effects for the increased temperature difference (Case G) . . . . . . . . 76

4.40 Mesh structure on the manikin and hood surface . . . . . . . . . 78

4.41 Predicted velocity and concentration fields at the hood face for Case D a) Complex manikin, b) Simple manikin . . . . . . . . . . . 78

4.42 Predicted velocity and concentration fields on the the manikin's surface for Case D a) Complex manikin, b) Simple manikin . . . . . 79

4.43 Predicted velocity and concentration fields on the vertical symmetry plane for Case D a) Complex manikin, b) Simple manikin . . . . . . 79

4.44 Comparison of concentration levels for Case D with complex and simple manikins . . . . . . . . . . . . . . 80

4.45 Time history of $z$-velocity for Case D a) Point 1, b) Point 2 . . . 80

4.46 Time history of concentration for Case D a) Point 1, b) Point $2 \ldots 80$

5.1 Domain decomposition. . . . . . . . . . . . . . . . . . 82 
5.2 Flowchart of the DREAM_P . . . . . . . . . . . . . . . 84

5.3 Two point data exchange with four points overlap paradigm . . . . 85

5.4 Schematic representation of flow between parallel plates . . . . . . 87

5.5 Time variation of streamwise velocity along the channel length at the centerline. . . . . . . . . . . . . . . . 88

5.6 Time variation of pressure along the channel length at the centerline. 88

5.7 Time variation of streamwise velocity along the channel height at the centerline. . . . . . . . . . . . . . . . . . . . . 89

5.8 Schematic view of the physical system along with anticipated vorticies. BL: Bottom Left, BR: Bottom right . . . . . . . . . . 90

5.9 Streamfunction contours obtained from the sequential and parallel runs with 4 processors . . . . . . . . . . . . . . . . 91

5.10 Comparison of velocity profiles obtained from sequential and parallel runs, a) $u$-velocity, b) $v$-velocity . . . . . . . . . . . . . . 91

5.11 Speed-up vs. number of processors for two grids . . . . . . . . . 93

5.12 Efficiency vs. number of processors for two grids . . . . . . . . 93

5.13 Speed-up vs. number of processors for different grids . . . . . . . . 94

5.14 Efficiency vs. number of processors for different grids . . . . . . . 94

5.15 Schematic of flow past a square cylinder [49] . . . . . . . . 96

5.16 Grid distribution on the $x y$-plane for flow past a square cylinder [49] 96

5.17 Contours of streamwise velocity in the center $x y$-plane a) instantaneous, b) mean . . . . . . . . . . . . . 98

5.18 Mean streamwise velocity along the centerline of square cylinder a) Present study, b) results from Rottach-Egern workshop (Reprinted from [63] with permission) . . . . . . . . . . . . . . . . 99

5.19 Time history of mean streamwise velocity at (1D, 0.5D) and corresponding PSD spectrum . . . . . . . . . . . 100

6.1 Variation of scalar quantity between adjacent cells . . . . . . . 103

6.2 Variation of scalar quantity within cells . . . . . . . . . . . . 104

6.3 Scalar fields for KIVA [68] a) Initial signal, b) Signal after 25 time steps . . . . . . . . . . . . . . . . . . . . . . . . . . . . .

6.4 Scalar fields for DREAM a) Initial signal, b) Signal after 25 time steps109

6.5 Velocity field (a), and initial scalar field (b) . . . . . . . . 110

6.6 Scalar field predicted using first order upwind scheme at the end of a) a quarter cycle, b) a half cycle . . . . . . . . . . . . . 111 
6.7 Scalar field predicted using QSOU scheme at the end of a) a quarter cycle, b) a half cycle . . . . . . . . . . . . . . . . . . 111

6.8 Passive scalar field on a fine mesh at the end of a) a quarter cycle $(\max =0.997), b)$ a half cycle $(\max =0.97) \ldots \ldots . \ldots 112$

7.1 Flow domain $(\Omega)$ with a circular immersed boundary $(\Gamma) \ldots \ldots .114$

7.2 Schematic of interpolation/extrapolation operation for a twodimensional case. (IB): immersed boundary point, (I,J) pairs: computational grid point . . . . . . . . . . . . 120

7.3 Computational domain along with the boundary conditions . . . . 123

7.4 Instantaneous streamwise velocity . . . . . . . . . . . . 125

7.5 Instantaneous passive scalar field, values less than 0.01 is cut off . . . 125

7.6 Time history of streamwise velocity at $(6 \mathrm{D}, 6.25 \mathrm{D}) \ldots \ldots \ldots$

8.1 Physical representation of the computational domain . . . . . . 126

8.2 Flow and concentration fields on the vertical symmetry plane of the

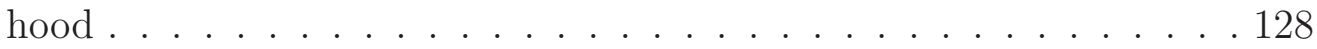

8.3 Instantaneous flow and concentration fields on the $x y$-planes a)

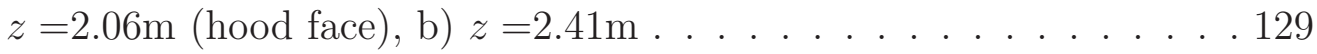

8.4 Instantaneous flow and concentration fields on the $x z$-planes a) $y=1.19 \mathrm{~m}, \mathrm{~b}) z=1.47 \mathrm{~m} \ldots \ldots . \ldots 129$

8.5 Time history of $z$-velocity at a point in the wake of the worker $(x=1.96 \mathrm{~m}, y=1.43 \mathrm{~m}, z=1.92 \mathrm{~m}) \ldots \ldots \ldots \ldots \ldots$

A.1 Unorganized point cloud obtained from 3D laser scanner . . . . . 150

A.2 Outlier points around the head and shoulder . . . . . . . . 150

A.3 A big hole on the surface . . . . . . . . . . . . . . . 151

A.4 Fixed head and shoulders . . . . . . . . . . . . . . . . . 151

A.5 Healed faceted geometry . . . . . . . . . . . . . . . . . 152

A.6 NURBS surfaces patched onto faceted geometry . . . . . . . . . 152 


\section{LIST OF SYMBOLS}

A Perpendicular area to the main flow direction, $\mathrm{m}^{2}$

$A^{+} \quad$ Van Driest constant

a Length of major axis of ellipse, $\mathrm{m}$

$c \quad$ Concentration, ppm; turbulence model constant

$C_{p} \quad$ Specific heat capacity at constant pressure, $\mathrm{J} / \mathrm{kg}-\mathrm{K}$

$C_{s} \quad$ Smagorinsky constant

$C_{\mu} \quad$ Model constant for $k-\epsilon$ turbulence model

$D \quad$ Diameter, m; weighting factor

$D_{\omega} \quad$ Cross-diffusion term in SST $k-\omega$ model

$E_{p} \quad$ Efficiency

$F \quad$ Convective flux

F $\quad$ Force field, $\mathrm{m} / \mathrm{s}^{2}$

$f \quad$ Any function, variable

$F_{1} \quad$ Blending function in SST $k-\omega$ model

$g_{i} \quad$ Gravitational acceleration, $\mathrm{m} / \mathrm{s}^{2}$

G Generation

$G_{\Delta} \quad$ Filter function

Gr Grashof number

$H \quad$ Height, $\mathrm{m}$

$h \quad$ Grid size, $m$

$J \quad$ Diffusion flux, $\mathrm{kg} / \mathrm{m}^{2}$-s

$k \quad$ Turbulent kinetic energy, $\mathrm{m}^{2} / \mathrm{s}^{2}$; thermal conductivity, $\mathrm{W} / \mathrm{m}-\mathrm{K}$

$k_{\Delta} \quad$ Residual kinetic energy, $\mathrm{m}^{2} / \mathrm{s}^{2}$

$l \quad$ Integral scale of turbulence, $\mathrm{m}$

$l_{s} \quad$ Smagorinsky length scale, $\mathrm{m}$

L Length; Characteristic length, $\mathrm{m}$

$L_{i j} \quad$ Germano identity (resolved turbulent stress tensor)

$M_{i j} \quad$ Scaled composite strain rate tensor

$\dot{m} \quad$ Mass flow rate, $\mathrm{kg} / \mathrm{s}$

$N_{b} \quad$ Total number of immersed boundary points affecting cell i,j,k 


\begin{tabular}{|c|c|}
\hline$n$ & Normal direction \\
\hline$p$ & Static pressure, $\mathrm{kg} / \mathrm{m}-\mathrm{s}^{2}$, number of processor \\
\hline$P e$ & Peclet number \\
\hline$r$ & Radius, m \\
\hline$R$ & Term for rapid strain modification in RNG $k-\epsilon$ turbulence model \\
\hline$R_{k}$ & $\mathrm{SST} k-\omega$ model parameter \\
\hline$R e$ & Reynolds number \\
\hline$R i$ & Richardson number \\
\hline$S$ & Source term \\
\hline$S_{c}$ & Constant part of source term \\
\hline$S_{p}$ & Speed-up \\
\hline$\overline{S_{i j}}$ & Characteristic filtered strain rate, $\mathrm{kg} / \mathrm{m}-\mathrm{s}^{2}$ \\
\hline $\bar{S}$ & Magnitude of filtered strain rate, $\mathrm{kg} / \mathrm{m}-\mathrm{s}^{2}$ \\
\hline$s$ & Distance, $\mathrm{m}$ \\
\hline$S r$ & Strouhal number \\
\hline$T$ & Temperature, K; time, s \\
\hline$T_{1}$ & Execution time of sequential code, $\mathrm{s}$ \\
\hline$T_{p}$ & Execution time of parallel code on $p$ processors, $\mathrm{s}$ \\
\hline$t$ & Time, s \\
\hline$U$ & Instantaneous streamwise velocity, $\mathrm{m} / \mathrm{s}$ \\
\hline$u_{i}$ & Mean velocity component, m/s \\
\hline$u_{i}^{\prime}$ & Fluctuating velocity component, m/s \\
\hline$U_{0}$ & Inlet velocity, $\mathrm{m} / \mathrm{s}$ \\
\hline$U_{1}$ & Outlet velocity, m/s \\
\hline$V$ & Tangential velocity, m/s \\
\hline$v$ & Instantaneous vertical velocity, m/s \\
\hline $\mathbf{v}$ & velocity vector for immersed boundary, $\mathrm{m} / \mathrm{s}$ \\
\hline$w$ & Instantaneous spanwise velocity, m/s \\
\hline$x$ & Spatial coordinate, axial distance, $\mathrm{m}$ \\
\hline$X_{r}$ & Recirculation zone length, m \\
\hline $\mathbf{x}_{\mathrm{s}}$ & Coordinates of point defining immersed boundary \\
\hline$Y$ & Dissipation \\
\hline$y$ & Vertical distance, $\mathrm{m}$ \\
\hline$y^{+}$ & Non-dimensional distance from the wall \\
\hline$z$ & Spanwise distance, $\mathrm{m}$ \\
\hline
\end{tabular}




\section{Greek Letters}

$\alpha \quad$ Constant gain in feedback forcing; turbulence model parameter

$\beta \quad$ Constant gain in feedback forcing; thermal expansion coefficient, $1 / \mathrm{K}$; turbulence model constant

$\delta_{i j} \quad$ Kronecker delta

$\Delta \quad$ Macroscopic differential operator; filter width, $\mathrm{m}$

$\Delta y_{1} \quad$ Distance between the wall and the first (adjacent) cell center, $\mathrm{m}$

$\epsilon \quad$ Turbulent dissipation rate, $\mathrm{m}^{2} / \mathrm{s}^{3}$

$\eta \quad$ The Kolmogorov length scale, $m$; turbulence model constant

$\Gamma \quad$ Diffusivity, $\mathrm{m}^{2} / \mathrm{s}$; immersed boundary

$\mu \quad$ Dynamic viscosity, $\mathrm{kg} / \mathrm{m}-\mathrm{s}$

$\nu \quad$ Kinematic viscosity, $\mathrm{m}^{2} / \mathrm{s}$

$\Omega \quad$ Flow domain

$\omega \quad$ Specific dissipation rate; angular velocity

$\Omega_{\Delta} \quad$ Support of the filter

$\phi \quad$ Passive scalar

$\rho \quad$ Density, $\mathrm{kg} / \mathrm{m}^{3}$

$\sigma \quad$ Prandtl number

$\tau_{i j}^{R} \quad$ Residual stress tensor, $\mathrm{kg} / \mathrm{m}-\mathrm{s}^{2}$

$\tau_{i j}^{r} \quad$ Deviatoric part of residual stress tensor, $\mathrm{kg} / \mathrm{m}-s^{2}$

$\theta_{s} \quad$ Angle of separation, degree

\section{Superscripts}

$0 \quad$ Old time level

$n \quad$ Index for time-level

$\max \quad$ Maximum

\section{Subscripts}

0 Initial, reference, inlet

A Apparent

a Cell face

b Buoyancy

d Number of dimensions

eff Effective 
FT Flow-through

$i, j, k \quad$ Index for spatial coordinate of interest

$m \quad$ Molecular

n Numerical

$P \quad$ Constant pressure

ref Reference

$s \quad$ Shoulder

$t \quad$ Turbulent

$x \quad$ X-direction

y $\quad \mathrm{y}$-direction

z $\quad$ z-direction

\section{Abbreviations}

CAD Computer-Aided Design

CFD Computational Fluid Dynamics

CFL Courant number

CMM Coordinate Measuring Machines

DDT Domain Decomposition Technique

DNS Direct Numerical Simulation

FFT Fast Fourier Transform

FVM Finite Volume Method

HPC High Performance Computing

IB Immersed Boundary

ITM Implicit Turbulence Modeling

LES Large Eddy Simulation

LEV Local Exhaust Ventilation

MPI Message Passing Interface

MUSCL Monotone Upstream-Centered Schemes for Conservation Laws

NURBS Non-Uniform Rational B-Spline

PDE Partial Differential Equation

PID Proportional-Integral-Derivative

PIV Particle Image Velocimetry

PSD Power Spectral Density

QUICK Quadratic Upwind Interpolation for Convective Kinematics 
QSOU Quasi-Second Order Upwind

RAM Random-Access Memory

RANS Reynolds-Averaged Navier-Stokes

RNG Renormalization Group

SGS Subgrid-Scale

SMPD Single Program Multiple Data

SST Shear-Stress Transport

TKE Turbulent Kinetic Energy

URANS Unsteady Reynolds-Averaged Navier-Stokes 


\section{Chapter 1}

\section{Introduction}

Effective Local Exhaust Ventilation (LEV) systems are critical in protecting workers from potentially hazardous materials that may become airborne while performing routine procedures. The key concept underlying LEV is the capture of airborne contaminants at the source [1] and their safe removal from the working environment. In many instances, extraneous factors, such as crossdrafts and work practices, make this task difficult. Thus the success or failure depends not only on the design of exhaust hood but also on the strength of extraneous factors.

An exhaust hood is a LEV tool that pulls the air from the ambient environment and carries away the contaminants released from the process with

it. If the contaminants are released from a process inside the hood, those hoods are classified as enclosing hoods. Depending on the purpose of usage, a number of different enclosing hood designs are available. Among them, benchtop enclosing hoods, without sash, are widely used in industry. Due to increasing concerns with fugitive emission in work places and worker exposure, transport of the airborne contaminants inside and around the enclosing hoods has recently received considerable research interest.

Although experimental investigations provide quite a bit of very reliable and useful information about the airflow, they may fall short in providing all of the required information about the phenomena. This is especially true 
with regard to measurements of instantaneous distribution of contaminant. On the other hand, numerical analyses when validated against experiments, are considered to be very useful for providing complete and detailed information that help researchers/engineers understand the phenomena better and design more effective tools. Furthermore, its higher degree of flexibility and lower cost makes numerical analyses very popular.

The main focus of the present work is mainly on understanding the flow dynamics in the near wake of the worker standing in front of an enclosing benchtop hood and investigating the influence of extraneous factors on the exposure level utilizing Computational Fluid Dynamics (CFD) technique. However, in the course of the work, efforts have also been spent on in-house CFD code development and experimental flow visualization.

This chapter provides an introduction to the flows associated with enclosing bench-top hoods, and to the numerical modeling along with the critical issues for reliable simulations.

\subsection{Flows Associated with Bench-top Enclosing Hoods}

It is generally known that bench-top enclosing hoods with reasonable levels of airflow can drastically reduce exposure of workers to potentially hazardous airborne contaminants. The effectiveness of these hoods is dictated by the airflow dynamics, the hood geometry, and the rate of generation of contaminants. Even though it is commonly assumed that higher face velocities $\left(V_{\text {Face }}\right)$ and smoother airflow lead to higher level of contaminant removal, the relationship between those parameters and hood effectiveness has not been well established [2]. Indeed, it is possible that high suction rates may not be desirable [1] due to their incidental negative effects on the process, contaminant generation, and the higher energy consumption, which, in turn, will result in higher operating costs [3]. Furthermore, increased suction rate results in higher level of turbulence, and hence, stronger diffusion of contaminants at the hood face. In general, all these factors keep workers from using high suction rates. Therefore, 
flows associated with enclosing hoods are still quite weak and vulnerable to unfavorable environmental conditions, such as cross-drafts and disturbances created by the presence of a worker, or opening of an outside door.

The presence of a worker standing in front of a hood, which acts like a contraction, also introduces additional complexity to the airflow. In the way it has been described above, the flow under consideration can be classified as an accelerating/converging flow past a bluff body, since the worker's torso acts as an obstacle to the flow drawn into the hood. Although a large body of research exists on the free-stream flows around bluff bodies, only a few studies $[4,5]$ have been reported in the literature regarding the effects of suction on the flow around bluff bodies. However, based on the common features of both cases, it is still possible to presume some characteristics of the flow under consideration.

A common approach used in the numerical studies is to represent a worker as an elliptical cylinder [4] with an aspect ratio of 0.5. Based on the typical airflow velocity $(0.05-5 \mathrm{~m} / \mathrm{s})$ and the shoulder diameter of the worker (larger diameter of the elliptical cylinder; $0.3-0.6 \mathrm{~m}$ ), the typical Reynolds number $(R e)$ in such flows is possibly in the range of 1,000 - 200,000 [4]. For this range of Reynolds numbers, it is known that the flow regime around the an elliptical cylinder immersed in a free-stream encompasses the subcritical regime [6], with characteristic features of separation and vortex shedding. Despite the fact that the Reynolds number marking the borders between the regimes can significantly vary due to the effects of contraction/acceleration and all other factors, such as the blockage effect of the hood, it would not be too unrealistic to expect similar flow structures in the near wake of the worker. Indeed, experimental studies [7-11] indicate formation of unsteady flow structures in front of the worker.

Although the reported typical airflow velocities range up to $5 \mathrm{~m} / \mathrm{s}$ in the literature, in the present study, maximum cross-draft velocity and face velocity used are $0.29 \mathrm{~m} / \mathrm{s}$ and $1.00 \mathrm{~m} / \mathrm{s}$, respectively. These low velocities result in a low turbulence flow. On the other hand, acceleration of the flow into the hood 
at higher suction rates is expected to give rise to re-laminarization of the flow and/or suppression of the formed vortices in the worker's wake. Hence, a complex combination of all of the flow regimes, spanning from laminar to turbulent, can take place under transition or concurrently at different locations of the flow. Thus, the entrainment of contaminants within the working environment or breathing zone of the worker are governed by flow dynamics like vortex shedding and turbulent diffusion, simultaneously.

Another important factor that must be taken into account is the buoyancy effects due to worker's body heat [12]. For the problem under consideration, depending on the relative strength of cross-draft to suction rate, there might be cases where buoyancy effects overshadows or at least perturbs the primary flow patterns in front of the worker. These cases can be determined theoretically from a dimensionless quantity called the Richardson number, Ri. The Richardson number, defined as the ratio of Grashof number, $G r$, to Reynolds number, $R e$, squared $\left(G r / R e^{2}\right)$ is used to determine the relative importance of the buoyancy force to the viscous force acting on the fluid, in other words the importance of diffusion by natural convection relative to the forced convection. When this number exceeds unity $(R i>>1)$, one should expect significant buoyancy contributions to the flow. For the present problem, based on the air flow rates used and the experimentally measured temperature difference (3-10K) between the human skin (or clothing) and his surrounding environment, the Richardson number can take values ranging from about 2.22 to 20.87 , indicating the possible existence of strong buoyancy effects.

This complicated and transitional/turbulent nature of the flow occurring between the worker and hood, necessitates a good understanding of the underlying flow dynamics, not only for accurate prediction of flow structures, but also for optimal adjustments of parameters that can possibly affect the effectiveness of the hood. Extensive experimental and theoretical research has been performed on airflow into LEV tools. With the rapid advance in computer technology, CFD has become a standard in analysis of LEV tools due to its 
ability to provide richer quantitative details about the unsteady flow features and turbulent transport as well as higher degree of flexibility and a lower cost relative to experimental studies. However, the reliability of results from a CFD analysis depends on many factors. Among them, the proper selection of turbulence models, grid structure, and numerical schemes are the key issues that will directly affect the simulation accuracy.

\subsection{Turbulence Modeling}

In the current problem of interest, the flow can be in different states at different locations. Also, strong self-induced unsteadiness involving separation, vortex shedding, and turbulent mixing are anticipated. Since the distinction between laminar, transitional, and turbulent flow is difficult, the simplest way around this problem is to calculate the flow using a turbulence model that is capable of capturing turbulent transitions. The turbulent kinetic energy should be approximately zero in the nominal laminar flow regimes [13].

Numerical solution techniques employed in CFD for turbulent flows can be broadly classified into three categories: Direct Numerical Simulation (DNS), Large Eddy Simulation (LES), and Reynolds-Averaged Navier-Stokes (RANS) simulation. DNS, conceptually the simplest approach, consists in solving the Navier-Stokes equations without any approximations, and resolving all of the spatial and temporal scales of the turbulence, from the smallest dissipative scales, $\eta$ (Kolmogorov scales), to the integral scale, $l$ (case characteristic length scale) $[14,15]$. As a result, DNS requires a very fine grid resolution and small time steps, which, in turn, leads to a prohibitively high computational cost for its application to large-scale engineering flows. In LES, the larger three-dimensional unsteady turbulent motions are directly represented, whereas the effects of the smaller scale motions are modeled [15]. This eliminates the need for the very fine spatial grid and small time steps of DNS, and hence, leads to a relatively affordable computational cost. LES has been becoming quite popular due to its high accuracy for flows in which large scale unsteadiness is significant - such 
as the flow over bluff bodies, which involves separation and vortex shedding. RANS simulation, which is the most practical and commonly used approach due to its low computational cost, is carried out by solving the time-averaged Navier-Stokes equations closed with turbulence models which are mainly based on eddy-viscosity or Reynolds-stress modeling concepts. The major drawbacks of the RANS approach are that they rely on the turbulence models which are not universal and employ sets of empirical parameters which will not work for all applications. Furthermore, it is not trivial to provide general rules and recommendations for the selection and use of turbulence models for complex applications. Therefore, selecting the optimal model and the model constants for a particular application is generally a major challenge, and nonetheless, it is crucial for reliable RANS calculations.

One common aspect of RANS simulation and LES, which are employed

in this study, is that they both use eddy-viscosity concept in one form or another. In this way, the effect of smaller scale motions present in the flow, but not resolved by the grid, are accounted for by calculating a local turbulent viscosity and adding it to the laminar (molecular) viscosity to give an effective viscosity at each cell. When appropriately used, this technique should still produce reasonably accurate results despite higher values of viscosity. Eulerian simulations of turbulent flows still suffer from the over-prediction of the viscosity. At this point one should look to address the numerical errors to overcome this problem.

\subsection{Numerical Issues}

The difficulty in Eulerian simulations of the unsteady, separated, and turbulent flows lies in their high sensitivity to the dissipative nature of the computational grid and the numerical scheme used for discretization of convection terms in the momentum equations, among other errors such as phase or dispersion error and aliasing errors. The numerical schemes are inherently open to error that manifests itself as numerical diffusion, which is 
a smoothing effect that smears out the gradient in the flow leading to 'diffusion' of momentum in the direction of the flow. Factually, the numerical diffusion does not behave like a true viscous diffusion because it is primarily associated with fluid convection and does not possess the correct stress-versus-strain-rate dependency associated with the real viscosity [16]. The numerical viscosity, $\nu_{N}$, can roughly be formulated as:

$$
\nu_{n}=c U h
$$

in order to quantify its extent. In Equation 1.1, $c$ is a constant determined by the numerical scheme. $U$ is the fluid velocity and $h$ is the length scale representing the grid size. According to both this relation and theory, smaller grid size and higher order schemes tend to yield smaller numerical viscosities. However, using high order schemes is not always the right procedure to minimize the numerical viscosity since stability problems may arise in solution. Nevertheless, use of very fine grids ( small $h$ ) always brings in extra computational burden. The only way around this problem might be to use a numerical scheme with reasonable accuracy and a fine enough grid to yield low numerical diffusion and to capture all important flow features. When these conditions are not met, numerical viscosity dominates the viscous forces and the simulation becomes untrustworthy due to the significant decrease in the apparent Reynolds number, $R e_{A}$, which can be defined as

$$
R e_{A}=\frac{U L}{\nu_{e f f}}
$$

where $U$ and $L$ are the velocity of the fluid, and the characteristic length of the body, respectively. Effective viscosity, denoted by $\nu_{\text {eff }}$ can be defined as

$$
\nu_{e f f}=\nu_{m}+\nu_{t}+\nu_{n}
$$


where $\nu_{l}, \nu_{t}$, and $\nu_{n}$ denote molecular (laminar), turbulent, and numerical viscosities, respectively. The low order schemes lead to higher values of $\nu_{n}$ resulting in over-prediction of $\nu_{e f f}$, and hence, lower $R e_{A}$. For flows, especially in critical regime, a substantial decrease in the apparent Reynolds number, $R e_{A}$,

due to an increase in the effective viscosity may lead to a change in flow regime and prevent capturing the correct physics in the simulations [17].

\subsection{Literature Review}

Recently, transport of airborne contaminants inside and around the benchtop enclosing hoods has received considerable research interest, due to increasing concerns with fugitive emissions and worker exposure. Much of the effort has been devoted to understanding flow dynamics specifically inside the enclosing hoods. However, some research attention has been given to the airflow in the wake of a worker standing in front of an enclosing hood.

One of the pioneering works on the worker exposure near an enclosing hood belongs to Ljungqvist [7]. In his experimental investigation with flow visualization methods, Ljungqvist demonstrated that the wake effect is capable of destroying the beneficial effect of the ventilation system. Also, his study visually revealed that the contaminants in the fume hood can be discharged outward through the opening and may be inhaled by the worker or by the others in the area. Such discharge can be caused by periodic or unstable vortices or by large scale turbulence in an unstable wake region in front of a worker. Later, Ljungqvist [8] supported his findings with a quantitative tracer gas measurement and showed that outward discharge takes place even at the smallest possible openings as soon as a worker uses the fume hood.

In a two-dimensional numerical study, Dunnett [4] made an investigation into the effects of the distance between worker and the exhaust opening on the wake flow for different Reynolds numbers ranging from 2,000 to 200,000. He predicted the flow field using standard $k-\epsilon$ turbulence model for higher Reynolds numbers and used small artificial perturbations to start the shedding 
process around the worker. The results showed that if the worker is situated some distance away from the opening (hood face), then the flow field is unsteady with vortex shedding occurring in the worker's wake. However, if the worker is close to the exhaust opening then the flow is steady and a very small area of recirculating flow exists in the wake of the worker.

Recently, Lan and Viswanathan [18] studied the airflow around a rounded body standing in front of a variable volume/constant face velocity enclosing fume hood. They performed both two- and three-dimensional computations with standard $k-\epsilon$ turbulence model and demonstrated the flow fields and profiles in various vertical planes and a main vortex behind the sash. Their results indicated that there is no occurrence or recirculation leading to concentration buildup in the region between the worker and the hood.

In a three-dimensional CFD study, $\mathrm{Hu}$ et al. [19] investigated the effect of exterior obstructions of different shapes and positions on flow patterns inside and around the fume hood. Both laminar and turbulent flows were considered. None of the obstructions investigated created a recirculation zone in their wake that can lead to fluid leakage from the opening of the hood.

Altemose et al. [9] performed tracer gas tests with both a human subject and a manikin (as a surrogate for the human subject) standing in front of a laboratory-type enclosing fume hood with horizontally sliding sashes. Aside from investigating the effect of the sash configurations, they also applied a leakage test based on the detection limit of a Leakmeter. The data collected indicated some outward leakage, mainly because of the unstable vortices formed in the wake of the human subject and the manikin. Moreover, they found that the face velocity and its variability as well as the magnitude of cross-drafts relative to face velocity are important variables in determining whether a hood will leak. Interestingly, the temporal variation of the face velocity was more strongly related to the human subject tracer gas results than spatial variation, a parameter traditionally believed to be more important. 
Nicholson et al. [20] conducted a CFD analysis with the commercial software 'Flovent v1.4' for assessing the performance of an aerodynamic fume hood. They used standard $k-\epsilon$ model and a sharp-edged manikin representing the worker. Their simulation indicated that the presence of the manikin disturbs the airflow at the hood face. Removal of the rear baffle and lipfoil resulted in a much greater effect on the airflow due to the worker. Recirculation zones became dominant both behind the sash and across the work surface, and a large volume of air flowed from the back of the working volume to the hood face. Without the rear baffle or the lipfoil, concentrations reached several orders of magnitude higher at the hood face. However, the conclusions stated by the authors are not clearly supported by the figures presented, since the velocity predictions, in almost all cases studied, seem to be positive in the wake, indicating a flow with no recirculation.

Tseng et al. [10] studied the complicated three-dimensional flow patterns and the real-time tracer gas $\left(S F_{6}\right)$ leakage via a laser-assisted flow visualization method and a standard/special gas sampling technique, respectively. Through flow visualization, large-scale vortex structures and boundary layer separation were observed around the side poles and doorsill of the hood. In the near-wake region of the manikin, large recirculation zones and wavy flow structures were also identified. When trace gas concentration measurements were conducted, they showed significant leaks near the sides of the manikin. These areas with high contaminant leaks exactly corresponded to where the flow recirculated or separated. The local structures were found to be more important than the global parameter of the face velocity when considering the overall leakage problem. They concluded that unsteady turbulent dispersion is one of the important causes for hood leakage.

Recently, Huang et al. [11] conducted a diagnostic study mainly on the aerodynamics of an air-curtain fume hood using the laser-light-sheet-assisted smoke flow visualization method. However, for comparison, they performed some tests for conventional hoods without sash, too. Their measurements indicated 
an increase in contaminant concentrations at the hood face in the presence of a worker. The leakage can be a result of both reverse flow and turbulent diffusion.

In another recent study, Chern and Cheng [21] performed a CFD analysis on turbulent diffusion of $S F_{6}$ in push-pull and exhaust fume hoods. Similar to aforementioned CFD studies, they used standard $k-\epsilon$ turbulence model. They also represented the worker's body as a sharp-edged block body, which is more prone to cause flow separation leading to formation of vortices in the near wake of the worker. Hence, different from previous CFD analyses, they stated that some recirculation zone was captured in their simulation. However, it is not very clear from their publication whether the reported vortices are induced by the flow separation around the worker or at the edge of bottom plate of the hood.

A summary of the literature reviewed above is given in Table 1.1

Table 1.1: Summary of the literature review on studies related to enclosing hoods

\begin{tabular}{|c|c|c|c|c|}
\hline Author & Basis & Model/Method & Sash & Findings \\
\hline Ljungqvist et al. [7] & Exp. & Smoke Test & Yes & $\begin{array}{l}\text { Periodic or unstable vortices } \\
\text { Large scale turbulence }\end{array}$ \\
\hline Ljungqvist et al. $[8]$ & Exp. & Tracer Gas & Yes & Contaminant detected at the hood face \\
\hline Dunnett et al. [4] & Num. & Std. $k-\epsilon$ & No & $\begin{array}{l}\text { Steady wake } \\
\text { Negligibly small recirculation zone }\end{array}$ \\
\hline $\mathrm{Hu}$ et al. [19] & Num. & Std. $k-\epsilon$ & Yes & $\begin{array}{l}\text { No recirculation zone in the wake } \\
\text { No leakage }\end{array}$ \\
\hline Altemose et al. [9] & Exp. & Tracer Gas & Yes & $\begin{array}{l}\text { Reverse flow at the hood face } \\
\text { Unstable wake } \\
V_{\text {Face }} \& \text { its temporal variability important }\end{array}$ \\
\hline Nicholson et al. [20] & Num. & Std. $k-\epsilon$ & Yes & Small Recirculation zone \\
\hline Lan and Viswanathan [18] & Num. & Std. $k-\epsilon$ & Yes & No recirculation zone in the wake \\
\hline Tseng et al. $[10]$ & Exp. & Smoke test & Yes & $\begin{array}{l}\text { Large recirculation zone } \\
\text { Leakage where flow recirculates or separates }\end{array}$ \\
\hline Huang et al. [11] & Exp. & Smoke test & Yes/No & Contaminant at the hood face \\
\hline Chern and Cheng [21] & Num. & Std. $k-\epsilon$ & Yes & Some recirculation in the worker's wake \\
\hline
\end{tabular}

Exp.: Experimental

Num.: Numerical

Std.: Standard

Although the insights gained from these studies are highly valuable in describing the flow with its general features, these studies would have been more complete if the thermal impact of the worker's body had been taken into account. It is apparent that consideration of the buoyancy effects is a necessary 
step in more closely representing the actual work environment. While there is insufficient information on the effect of buoyancy on the flow associated with the enclosing hoods, the literature regarding the buoyancy driven flow about a human body in a quiescent or low wind speed indoor environment is quite numerous.

It has been reported in the literature [22-24] that at moderate room temperatures and with ordinary levels of activity, the average person can generate up to 100 Watts of thermal energy. Usually, the transfer of this thermal energy (heat) from human body to the surrounding environment happens through a combination of radiation, convection, evaporation, and respiration. More precisely, in recent study, Murakami et al. [25] found that these mechanisms account for $38.1 \%, 29.0 \%, 24.2 \%$ and $8.7 \%$, of the total heat loss, respectively. In the experimental studies with human subjects, naturally, all possible modes of heat transfer take place. However, in most of the numerical studies published, the effect of radiation on the airflow is neglected. In principle, this is a valid assumption because air is not a strongly absorbing-emitting medium. More clearly, air does not participate in radiative heat transfer, and the radiative heat transfer occurs only from surface (body) to surface (surrounding walls). This leaves the convection as the dominant heat transfer mode between a human body and surrounding air. In a numerical study, Murakami [26] included effect of radiation in addition to sensible and latent heat loss by convection. His simulations revealed that the largest heat loss from the body is by radiation. Even so, the heat loss mentioned should be to the surrounding walls due the reasons mentioned above. However, this point is not clear from the publication.

The driving force of convection is the temperature gradient that arises from the temperature difference between the human body and the surrounding air. The convective heat transfer leads to formation of a thermal free-convection boundary layer about the human body. Initially this boundary layer (starting at the feet) is laminar. However, as the flow rises up the body, transition occurs and the upper body becomes enveloped in rising turbulent flow. As the flow 
rises further, it separates from the body and forms a recirculation zone over the human body. This phenomenon, called human thermal plume, has been observed in experiments using Schlieren photography [24, 27], using PIV measurements and CFD simulations [22]. As the warm plume moves upward, it accelerates due to buoyancy. Once it gets far from the body the mixing slows it down.

When there is a wind velocity, the problem becomes a forced-convection problem. One of the most comprehensive studies on the airflow patterns around a worker in low-speed flow has been conducted by Heist et al. [28], in which a laser Doppler anemometer was used to analyze the airflow patterns around a child-size manikin under several different conditions. Their measurements revealed that the presence of body heat leads to dramatic changes in the flow pattern. With the manikin at the room temperature, they observed two slowly recirculating eddies on the downstream side of the manikin. On the other hand, with the addition of body heat to the manikin, the flow pattern downstream of the manikin was found to change to a rising vertical plume with velocities on the order of $0.1 \mathrm{~m} / \mathrm{s}$. They concluded that this vertical plume can easily transport particulate matter into the breathing zone from near the floor, and therefore, suggested that it is important to consider body heat in low-speed wind environments. However, at higher wind speeds $(0.1-0.3 \mathrm{~m} / \mathrm{s})$, they observed replacement of vertical plume with two recirculating eddies, a pattern similar to that with unheated manikin. Their findings agree well with earlier studies carried out by Johnson et al. [29] and Murakami et al. [30].

Recently, Li et al. [12] investigated different factors affecting numerical prediction of worker exposure to gaseous contaminant in a ventilated space. Their predictions indicated that the heat flux may have a significant impact on exposure, especially when the convection induced by buoyancy dominates the flow. 


\subsection{Motivation and Objectives of the Study}

The literature review presented in the preceding section reveals that there are inconsistencies between the numerical and experimental findings from the viewpoint of critical flow phenomena taking place in the wake of a worker standing at an enclosing hood. These contradictory observations can be attributed to the physical differences (e.g., flow configuration, flow rate, etc.) in the cases investigated, as well as to the numerical factors (e.g., scheme, grid, turbulence modeling, etc.) utilized. Also it is apparent that there is a gap in the literature about the thermal impact of the worker's body on exposure levels. With this as background, the main objective of the present study is to investigate the effects of both physical and numerical factors on the flow dynamics and the associated contaminant transport taking place in the worker's wake. This was accomplished in three phases:

1. Investigation of the effect of RANS turbulence models on the accuracy of CFD solutions, and recommendation of a RANS turbulence model for this type of flows.

2. Investigation of the effect of physical factors, such as the draft intensity and its direction, and worker's body heat and shape, and comparison of the numerical results with the experimental observations.

3. Development of a computational tool to handle similar large-scale, dynamic, bluff-body dominated flows. This phase involves the following tasks:

- Parallel implementation of DREAM, an in-house CFD code with LES capability.

- Implementation of the Immersed Boundary (IB) method into the parallel DREAM to account for solid bodies in the flow. 
- Implementation of the strongly monotone, Quasi-Second Order Upwind (QSOU) convection scheme into scalar transport solver of the DREAM.

- Validation and verification of the same code. 


\section{Chapter 2}

\section{Governing Equations and Turbulence Models}

The laminar to turbulent flow characteristics in separated flows (e.g., flow around a human body) are very intricate and impose significant challenges on their simulations. As stated previously, the simplest way around this problem is to calculate the flow as a turbulent one. For the investigation of indoor flows and worker exposure, the mean values of the flow variables are more useful than instantaneous turbulence variables [14]. Therefore, the interest is stronger in solving computationally more economic unsteady RANS equations with appropriate turbulence models. However, RANS turbulence models are not always successful in modeling large-eddy motion which is the primary mechanism for turbulent transport. LES, on the other hand, is clearly a superior method that can capture the flow dynamics, especially in separated flows. In the present work, both unsteady RANS and LES methods are used. In what follows, the governing equations, along with a brief introduction to the RANS turbulence models and LES, are presented.

In tensor notation, incompressible unsteady RANS (URANS) equations for the conservation of mass and momentum are written as follows:

Continuity Equation:

$$
\frac{\partial \bar{u}_{i}}{\partial x_{i}}=0
$$


Momentum Transport Equation:

$$
\begin{aligned}
\rho\left(\frac{\partial \bar{u}_{i}}{\partial t}+\bar{u}_{j} \frac{\partial \bar{u}_{i}}{\partial x_{j}}\right) & =-\frac{\partial \bar{p}}{\partial x_{i}}+\frac{\partial}{\partial x_{j}}\left(\mu \frac{\partial \bar{u}_{i}}{\partial x_{j}}\right)-\frac{\partial}{\partial x_{j}}\left(\rho \overline{u_{i}^{\prime} u_{j}^{\prime}}\right) \\
& +\rho_{0} g\left(1-\beta\left(T-T_{0}\right)\right)
\end{aligned}
$$

where $\bar{u}_{i}$ denotes mean velocity components. The fluctuating velocities are represented by $u_{i}^{\prime}$ and the overbar indicates time average. The mean pressure and the density are denoted by $p$ and $\rho$, respectively. The gravitational force and the buoyancy force acting on the fluid are combined using the Boussinesq approximation [31]; $\rho=\rho_{0} g\left(1-\beta\left(T-T_{0}\right)\right)$ to eliminate $\rho$ from the buoyancy term. In this term, $\rho_{0}$ is the constant density of the flow, $T_{0}$ is the operating temperature, and $\beta$ is the thermal expansion coefficient of the flowing medium at the operating temperature. The Boussinesq approximation is capable of producing accurate results provided that the variations in actual density are small; specifically, it is valid when $\beta\left(T-T_{0}\right)<<1$. This condition holds for the present problem.

In the present study, the contaminant specie is treated as a passive scalar. Thus, the following generic passive scalar transport equation is solved for the contaminant transport. It might be more convenient to consider the variable $\bar{\phi}$ as the mean concentration of the contaminant specie.

Passive Scalar Transport Equation:

$$
\frac{\partial \bar{\phi}}{\partial t}+\bar{u}_{j} \frac{\partial \bar{\phi}}{\partial x_{j}}=\frac{\partial}{\partial x_{j}}\left(\Gamma_{m} \frac{\partial \bar{\phi}}{\partial x_{j}}\right)-\frac{\partial}{\partial x_{j}}\left(\overline{\phi^{\prime} u_{j}^{\prime}}\right)
$$

where $\bar{\phi}$ and $\phi^{\prime}$ denote mean and fluctuating concentrations, respectively. $\Gamma_{m}$ is the molecular (laminar) diffusivity of the specie. Analogous to the turbulent momentum transport, the turbulent mass transport is assumed to be approximated by the eddy-diffusivity, $\Gamma_{t}$ concept.

$$
\overline{\phi^{\prime} u_{j}^{\prime}}=\Gamma_{t} \frac{\partial \bar{\phi}}{\partial x_{j}}
$$


where $\Gamma_{t}$ denotes the eddy (turbulent) diffusivity, and similar to the eddy (turbulent) kinematic viscosity $\left(\nu_{t}\right)$ it is not a fluid property but depends on the local state of turbulence. The Reynolds analogy between mass transport and momentum transport suggest that $\Gamma_{t}$ and $\nu_{t}$ are closely related by the following relation:

$$
\Gamma_{t}=\nu_{t} / S c_{t}
$$

where $S c_{t}$ is called the Schmidt number in mass transfer. The value of $S c_{t}$ is an important parameter as it determines how much the contaminant will diffuse. Experiments have shown that $S c_{t}$ is nearly constant with typical values of 0.7 1.0. In the present study the value of $S c_{t}$ was taken as 0.7 .

To account for the heat transfer from the worker's body to the surrounding air, the following equation for the conservation of energy is solved.

Energy Equation:

$$
\rho C_{p}\left(\frac{\partial T}{\partial t}+\bar{u}_{j} \frac{\partial T}{\partial x_{j}}\right)=\frac{\partial}{\partial x_{j}}\left(k_{e f f} \frac{\partial T}{\partial x_{j}}\right)
$$

In Equation 2.6, $T$ is the temperature, $k_{e f f}$ represents the effective thermal conductivity of the air, which is defined as the sum of molecular thermal conductivity, $k_{m}$, and turbulent thermal conductivity $k_{t}\left(:=C_{p} \mu_{t} / \sigma_{t}\right) . \quad C_{p}$ is the specific heat at constant pressure and $\sigma_{t}$ is the turbulent Prandtl number.

\subsection{RANS Turbulence Models}

Equations 2.1 and 2.2 are not closed due to the existence of more unknowns than the available equations. In order to close the set of equations given

above, the Reynolds stresses, $\left(\rho \overline{u_{i}^{\prime} u_{j}^{\prime}}\right)$, appearing on the right hand side of the momentum equation (Eq. 2.2) are modeled. The present study uses standard $k-\epsilon$, the Renormalization Group (RNG) $k-\epsilon$, and Shear-Stress Transport (SST) $k-\omega$ models which are based on the Boussinesq's eddy diffusivity hypothesis 
[32] to relate the Reynolds stresses to the mean velocity gradients as follows:

$$
\overline{u_{i}^{\prime} u_{j}^{\prime}}=\nu_{t}\left(\frac{\partial \bar{u}_{i}}{\partial x_{j}}+\frac{\partial \bar{u}_{j}}{\partial x_{i}}\right)-\frac{2}{3} k \delta_{i j}
$$

Here, $k$ is the turbulent kinetic energy and $\delta_{i j}$ is the kronecker delta. The advantage of this approach is the relatively low computational cost associated with the computation of the turbulent viscosity, $\nu_{t}$. In the case of the $k-\epsilon$ and $k-\omega$ group models, two additional transport equations (for the turbulent kinetic energy, $k$, and either the turbulent dissipation rate, $\epsilon$, or the specific dissipation rate, $\omega$ ) are solved and $\nu_{t}$ is computed as a function of $k$ and $\epsilon$ or $\omega$. The disadvantage of the Boussinesq hypothesis as presented is that it assumes $\nu_{t}$ is an isotropic scalar quantity, which is not factually true.

\subsection{1 $k-\epsilon$ Group Models}

The $k-\epsilon$ group models are semi-empirical models based on the following model transport equations for the turbulence kinetic energy, $k$, and its dissipation rate, $\epsilon$

$$
\begin{gathered}
\frac{\partial k}{\partial t}+\bar{u}_{i} \frac{\partial k}{\partial x_{i}}=\frac{\partial}{\partial x_{j}}\left(\Gamma_{k} \frac{\partial k}{\partial x_{j}}\right)+S_{k} \\
\frac{\partial \epsilon}{\partial t}+\bar{u}_{i} \frac{\partial \epsilon}{\partial x_{i}}=\frac{\partial}{\partial x_{j}}\left(\Gamma_{\epsilon} \frac{\partial \epsilon}{\partial x_{j}}\right)+S_{\epsilon}
\end{gathered}
$$

\subsubsection{Standard $k-\epsilon$ Model}

In the standard $k-\epsilon$ model [33], the effective diffusivities $\left(\Gamma_{k}\right.$ and $\left.\Gamma_{\epsilon}\right)$ appearing in Equations 2.8 and 2.9 are defined as

$$
\Gamma_{k}=\nu+\frac{\nu_{t}}{\sigma_{k}}
$$




$$
\Gamma_{\epsilon}=\nu+\frac{\nu_{t}}{\sigma_{\epsilon}}
$$

where $\sigma_{k}$ and $\sigma_{\epsilon}$ are the turbulent Prandtl numbers for $k$ and $\epsilon$, respectively. The $\nu$ and $\nu_{t}$ denote laminar (molecular) and turbulent (eddy) kinematic viscosities, respectively.

The turbulent (eddy) viscosity, $\nu_{t}$, is computed from the following expression which combines $k$ and $\epsilon$.

$$
\nu_{t}=C_{\mu} \frac{k^{2}}{\epsilon}
$$

In Equation $2.12 C_{\mu}$ is an empirical coefficient equal to 0.09 .

The modified source terms, $S_{k}$ and $S_{\epsilon}$, in Equations 2.8 and 2.9 are given by the following

$$
\begin{gathered}
S_{k}=G_{k}+G_{b}-\rho \epsilon \\
S_{\epsilon}=C_{1 \epsilon} \frac{\epsilon}{k}\left(G_{k}+C_{3 \epsilon} G_{b}\right)-C_{2 \epsilon} \rho \frac{\epsilon^{2}}{k}
\end{gathered}
$$

In the above equations, $G_{k}$ and $G_{b}$ represent the generation of turbulent kinetic energy due to the mean velocity gradients and buoyancy, respectively.

The contribution of buoyancy to the turbulence, $G_{b}$, is modeled as

$$
G_{b}=\beta g_{i} \frac{\mu_{t}}{\sigma_{t}} \frac{\partial T}{\partial x_{i}}
$$

where $g_{i}$ is gravitational acceleration in the $i$-direction. The present study uses a turbulent Prandtl number, $\sigma_{t}$ of 0.85 . The coefficient of thermal expansion, $\beta$, 
is defined as

$$
\beta=-\frac{1}{\rho}\left(\frac{\partial \rho}{\partial T}\right)_{p}
$$

In FLUENT, the buoyancy effects on the dissipation rate, $\epsilon$, are not accounted for, hence, $G_{b}$ is set to zero in Equation 2.14 .

Calculation of $G_{k}$ in Equation 2.13 can be found in FLUENT manual [34]. $C_{1 \epsilon}, C_{2 \epsilon}$ and $C_{3 \epsilon}$ are constants to be defined. In the present study, the default values of model constants were used:

$$
C_{1 \epsilon}=1.44, C_{2 \epsilon}=1.92, C_{3 \epsilon}=0.09, \sigma_{k}=1.0 \text { and } \sigma_{\epsilon}=1.3
$$

\subsubsection{The Renormalization Group (RNG) $k-\epsilon$ Model}

The RNG $k-\epsilon$ model has the same form as the standard $k-\epsilon$ turbulence model but includes some refinements on turbulent Prandtl numbers and differences in model parameters in the high-Reynolds-number limit. Besides these, another major difference between RNG $k-\epsilon$ and standard $k-\epsilon$ model is that there is an additional term in the $\epsilon$ equation for the RNG $k-\epsilon$ turbulence model, which accounts for the effect of the rapid strain. The RNG $k-\epsilon$ model is known to be more responsive to the effects of rapid strain and streamline curvature than the standard $k-\epsilon$ model. In this model, the modified source term, $S_{\epsilon}$, appearing in Equation 2.11 takes the following form:

$$
S_{\epsilon}=C_{1 \epsilon} \frac{\epsilon}{k}\left(G_{k}+C_{3 \epsilon} G_{b}\right)-C_{2 \epsilon} \rho \frac{\epsilon^{2}}{k}-R_{\epsilon}
$$

where

$$
R_{\epsilon}=\frac{C_{\mu} \rho \eta^{3}\left(1-\eta / \eta_{0}\right)}{1+\beta \eta^{3}} \frac{\epsilon^{2}}{k}
$$

Here, $\eta=S k / \epsilon, \eta_{0}=4.38$, and $\beta=0.012$. 
In the high-Reynolds-number limit, $\nu_{t}$ tends to Equation 2.12 with $C_{\mu}=$ 0.0845 derived using RNG theory [35]. The model constants $C_{1 \epsilon}$ and $C_{2 \epsilon}$ in Equation 2.14 have values derived analytically by the RNG theory. These values, used by default in FLUENT, are

$$
C_{1 \epsilon}=1.44, C_{2 \epsilon}=1.92
$$

The reader is referred to [34] for further details of the model including the calculation of inverse effective Prandtl numbers.

\subsubsection{Shear-Stress Transport (SST) $k-\omega$ Model}

Although this section is intended to provide an introduction to the ShearStress Transport (SST) $k-\omega$ model proposed by Menter [36], for the sake of clarity, the standard $k-\omega$ model [37] is presented first. In the standard $k-\omega$ model, the equation for $k$ is the same as the one used in $k-\epsilon$ model (Equation 2.8), whereas the $\epsilon$ equation is replaced by an equation for specific dissipation rate, $\omega$, which is defined as $\epsilon / k$.

$$
\begin{aligned}
& \frac{\partial k}{\partial t}+\bar{u}_{i} \frac{\partial k}{\partial x_{i}}=\frac{\partial}{\partial x_{j}}\left(\Gamma_{k} \frac{\partial k}{\partial x_{j}}\right)+S_{k} \\
& \frac{\partial \omega}{\partial t}+\bar{u}_{i} \frac{\partial \omega}{\partial x_{i}}=\frac{\partial}{\partial x_{j}}\left(\Gamma_{\omega} \frac{\partial \omega}{\partial x_{j}}\right)+S_{\omega}
\end{aligned}
$$

The key advantage held by the $\omega$ equation is that of accurately predicting the turbulent length scale in difficult-to-solve pressure gradient flows, leading to improved predictions of wall shear stress. Furthermore, provided that the near-wall mesh is sufficient, this model can be used to directly predict the lowReynolds number effects on the turbulence field in the near wall regions and has been proven to be more accurate in describing the transition from laminar to turbulent flow [38]. As the model employs a low-Reynolds number formulation, the requirement for using additional non-linear wall damping functions is made 
redundant. The calculation of the correct sublayer behavior is accomplished through the implementation of a simple Dirichlet-type boundary condition for $\omega$.

The modified source terms, $S_{k}$ and $S_{\omega}$, in Equations 2.19 and 2.20 are as follows

$$
\begin{aligned}
& S_{k}=G_{k}-Y_{k} \\
& S_{\omega}=G_{\omega}-Y_{\omega}
\end{aligned}
$$

where $G_{k}$ represents the generation of turbulent kinetic energy due to mean velocity gradient and $G_{\omega}$ represents the generation of $\omega . Y_{k}$ and $Y_{\omega}$ denote the dissipation of $k$ and $\omega$ due to turbulence, respectively. Calculation of these constants are available in FLUENT manual [34].

In the standard $k-\omega$ model, effective diffusivities appearing in Equations 2.19 and 2.20 are modeled as follows

$$
\begin{gathered}
\Gamma_{k}=\nu+\frac{\nu_{t}}{\sigma_{k}} \\
\Gamma_{\omega}=\nu+\frac{\nu_{t}}{\sigma_{\omega}}
\end{gathered}
$$

where $\sigma_{k}$ and $\sigma_{\omega}$ are the turbulent Prandtl numbers for $k$ and $\omega$, respectively. The turbulent viscosity $\nu_{t}$, is computed from

$$
\nu_{t}=\alpha^{*} \frac{k}{\omega}
$$

The damping of turbulent viscosity at low-Reynolds number is made through 
the coefficient $\alpha^{*}$ which is given by

$$
\alpha^{*}=\alpha_{\infty}^{*}\left(\frac{\alpha_{0}^{*}+R e_{t} / R_{k}}{1++R e_{t} / R_{k}}\right)
$$

where $R e_{t}=\frac{k}{\nu \omega}, R_{k}=6, \alpha_{0}^{*}=\beta_{i} / 3$ and $\beta_{i}=0.072$. Note that, in the highReynolds number of the $k-\omega$ model, $\alpha^{*}=\alpha_{\infty}^{*}=1$. Further details of the model can be found elsewhere [34].

The SST $k-\omega$ turbulence model, on the other hand, is a blend of the Standard $k-\omega$ model formulation in the near-wall region with the Standard $k-\epsilon$ formulation in the far field. In that sense, it can be considered as a zonal model. In order to ensure that the model equations behave appropriately in both the near-wall and far-field zones, a blending function that operates as a switch between the two models is used. Unlike the Standard $k-\omega$ model, the SST $k-\omega$ model modifies the turbulent viscosity by limiting the turbulent shear stress within the boundary layers.

The first step in blending of $k-\omega$ and $k-\epsilon$ models is to transform the latter into equations based on $k$ and $\omega$. The variations between this formulation and the Standard $k-\omega$ model are that an additional cross-diffusion term, $D_{\omega}$, is incorporated in the $\omega$ equation and the model constants are different. Some of the parameters appearing in the Standard $k-\omega$ model are multiplied by a so-called blending function $F_{1}$ and some parameters in the transformed $k-\epsilon$ model by $\left(1-F_{1}\right)$. The corresponding equations of each model are then added together. The blending function $F_{1}$ is designed to take a value of one in the sublayer and logarithmic region of the boundary layer (activating $k-\omega$ model) and to gradually switch to zero in the wake region. More clearly, the blending takes place in the wake region of the boundary layer [36, 39].

In the SST $k-\omega$ model the turbulent Prandtl numbers for $k$ and $\omega\left(\sigma_{k}, \sigma_{\omega}\right)$ are non-constants, in contrary to the Standard $k-\omega$ model in which they are constants. 
The SST $k-\omega$ model calculates the production of $k$ in the same way as per the Standard $k-\omega$ model, hence the $k-\epsilon$ type models, by Equation 2.19. However, it calculates the production of $\omega$ differently from the Standard $k-\omega$ model.

The modified source terms take the following forms

$$
\begin{gathered}
S_{k}=G_{k}-Y_{k} \\
S_{\omega}=G_{\omega}-Y_{\omega}+D_{\omega}
\end{gathered}
$$

The model constants applied in the high Reynolds number are as follows:

$$
\begin{gathered}
\sigma_{k, 1}=1.176, \sigma_{\omega, 1}=2.0, \sigma_{k, 2}=1.0, \sigma_{\omega, 2}=1.168 \\
\alpha_{1}=0.31, \beta_{i, 1}=0.075, \beta_{i, 2}=0.0828, \kappa=0.41
\end{gathered}
$$

For further details of the model the reader is directed to $[34,36,40]$.

\subsection{Large Eddy Simulation}

LES has been increasingly applied to model airflows in enclosed environments due to its ability to provide rich dynamic details as compared to RANS models, which are based on modeling all scales of motion present in a turbulent flow. The basic idea behind LES is to solve large-scale motions which can be represented by the computational grid and to model smaller scale motions, which are also known as subgrid-scale (SGS) motions. This approach enables resolving larger scales of motion which predominately contains the turbulent kinetic energy and anisotropy in a turbulent flow. The 'segregation' of large scales from small scales can be achieved through a filtering (space averaging) operation. Thus, LES solves filtered (transformed) Navier-Stokes equations for large-scales while modeling subgrid-scales.

Filtering of dependent variables (velocity components and pressure) in the Navier-Stokes equations is similar to the process of Reynolds averaging. Clearly, 
each variable, $f_{i}$, in the Navier-Stokes equation is decomposed into a local average, $\bar{f}_{i}$, and a subgrid-scale component, $f_{i}^{\prime}$, such that $\bar{f}_{i}+f_{i}^{\prime}=f_{i}$. The local averages, generated through spatial filtering, are the filtered (resolved) components. Mathematically, the filtering operator is a convolution integral of the form:

$$
\bar{f}(x, t)=\int_{\Omega} G_{\Delta}(x, y) f(y, t) d V
$$

where $G_{\Delta}$ is a filter function. There are several filters, such as box (top-hat) filter, Gaussian filter and spectral (or Fourier cut-off) filter, etc. An overview of filter and their properties can be found in [15]. The present study uses box filter of the form:

$$
G_{\Delta}(x, y)= \begin{cases}\frac{1}{\left|\Omega_{\Delta}(x)\right|} & \text { if } y \in \Omega_{\Delta}(x) \\ 0 & \text { elsewhere }\end{cases}
$$

where $\Omega_{\Delta}$ denotes the support of the filter function with a filter width of $\Delta:=\sqrt[d]{\left|\Omega_{\Delta}(x)\right|}$.

Once the Navier-Stokes equations are filtered, the resultant equations depend only on local averages. However, the convection term in the filtered equation is not closed. In order to eliminate this problem a residual tensor $\tau_{i j}^{R}:=\overline{u_{i} u_{j}}-\bar{u}_{i} \bar{u}_{j}$, representing the SGS stresses is introduced. A useful way to do this is to decompose the $\tau_{i j}^{R}$ into a deviatoric part and an isotropic part which will be absorbed into the pressure term. Briefly one can write

$$
\tau_{i j}^{R}=\tau_{i j}^{r}+\frac{2}{3} k_{\Delta} \delta i j
$$

where $k_{\Delta}$ is the residual kinetic energy defined as $k_{\Delta}=(1 / 2) \tau_{i i}^{R}$ 
With these modification, the filtered momentum equation takes the form:

$$
\frac{\partial \bar{u}_{i}}{\partial t}+\frac{\partial}{\partial x_{j}}\left(\bar{u}_{i} \bar{u}_{j}\right)=-\frac{\partial \bar{p}}{\partial x_{i}}+\frac{\partial}{\partial x_{j}}\left(2 \nu \bar{S}_{i j}\right)-\frac{\partial}{\partial x_{j}} \tau_{i j}^{r}
$$

where $\bar{S}_{i j}$ is the strain rate tensor for the resolved scale and is expressed by

$$
\bar{S}_{i j}=\frac{1}{2}\left(\frac{\partial \bar{u}_{i}}{\partial x_{j}}+\frac{\partial \bar{u}_{j}}{\partial x_{i}}\right)
$$

The magnitude of $\bar{S}_{i j}$, known as characteristic filtered strain rate, is quantified by:

$$
|\bar{S}|=\sqrt{2 \bar{S}_{i j} \bar{S}_{i j}}
$$

Note that both $\bar{S}_{i j}$ and $|\bar{S}|$ are two prominent quantities in the SGS modeling. Once the filtering operation is done, it remains to model the deviatoric part of the SGS stress tensor; $\tau_{i j}^{r}$. Smagorinsky [41] suggested the first SGS model. The model correlates eddy viscosity to the strain rate as follows:

$$
\tau_{i j}^{r}=-2 \nu_{t} \bar{S}_{i j}
$$

One can notice the similarity between Smagorinsky's hypothesis and the Boussinesq eddy viscosity concept. Analogous to the mixing length model, the eddy viscosity,$\nu_{t}$ is constructed from

$$
\nu_{t}=l_{s}^{2}|\bar{S}|=\left(C_{s} \Delta\right)^{2}|\bar{S}|
$$

where $l_{s}$ is the Smagorinsky length scale proportional to the characteristic filter width, $\Delta$. In the present study, close to the walls, the van Driest damping is used, defined by $l_{s}=C_{s} \Delta\left(1-e^{y^{+} / A^{+}}\right)$with $A^{+}=25 . C_{s}$ is the Smagorinsky constant whose value is usally between 0.05 and 0.25 , depending on characteristic of the flow. Lilly [42] suggested a value of 0.17 for $C_{s}$ in homogeneous isotropic 
turbulence. However, since $C_{s}$ is not a universal constant and needs to be tuned for different flows, many modifications and improvements to the original model were proposed thereafter.

Analyses by Voke [43], Meneveau and Lund [44] and Pope [15] show that, even in the isotropic turbulence, as $\Delta \rightarrow \eta, C_{s}$ must dynamically vary with $\Delta$ in order to reproduce the correct SGS dissipation rate in the viscous (dissipation) range. Thus, the dynamic Smagorinsky-Lilly model based on the Germano identity $[45,46]$ was proposed to compute the $C_{s}$ with the information provided from the resolved scales of motion as follows:

$$
\left(C_{s}\right)^{2}=\frac{\left\langle L_{i j} M_{i j}\right\rangle}{\left\langle M_{i j} M_{i j}\right\rangle}
$$

where $L_{i j}$ and $M_{i j}$ are the resolved stress tensors. In this form, Eq. 2.37 has some stability issues. A commonly adopted approach to stabilize the numerical approach is to take the average of both the numerator and the denominator of Eq. 2.37 in the homogeneous direction. The averaging operation helps preventing highly variable eddy-viscosity field with negative values produced by the dynamic model. However, the averaging operation is not easy to perform when the flow field does not have any statistical homogeneous direction [14]. Several remedies for this situation has been suggested by various researchers [47, 48]. A comprehensive overview on the dynamics models can be found in [49]. 


\section{Chapter 3}

\section{RANS Turbulence Model Performance}

Among various CFD influential factors, proper selection of a turbulence model is a key issue that will affect the simulation accuracy and efficiency. The purpose of this section is to test behaviors of several RANS turbulence model for the flow under consideration. For this purpose, a two-dimensional test case was adopted from the literature and simulations were performed using standard $k-\epsilon$, RNG $k-\epsilon$, and SST $k-\omega$ turbulence models to develop confidence in their use for further investigation.

\subsection{Two-dimensional Test Case}

The two-dimensional test case adopted in this part of the study is a simple conceptual model (Figure 3.1) originally proposed by Dunnett [4]. Simulations were run for Reynolds numbers in the range of $1.0 \times 10^{3}-1.0 \times 10^{5}$ to investigate the two-dimensional wake flow of an ellipse representing the worker. In this flow configuration, the center of the ellipse is $0.75 a$ away from the face of the exhaust opening, representing a hood, as shown in Figure 3.1.

Although this two-dimensional analysis is a crude approximation of the real situation (as already pointed out by Dunnett), George et al. [50] found in an experimental work that a two-dimensional approach may provide a reasonable approximation to the physics of the problem. To conform with Dunnett's study, identical flow configuration and boundary conditions were used. Accordingly, 


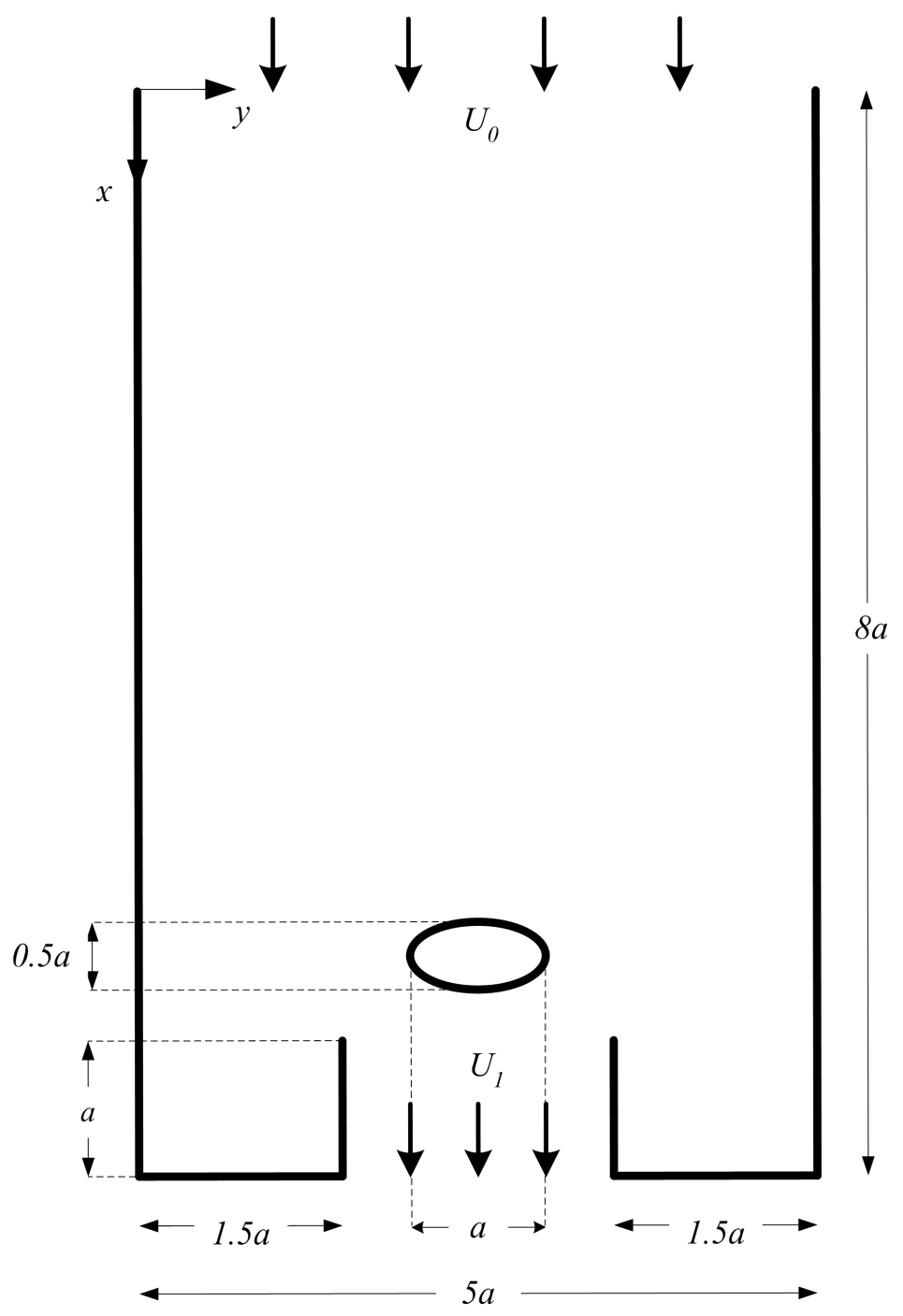

Figure 3.1: Schematic representation of the physical system

Reynolds number is defined as $R e=a U_{0} / 2 \nu_{\text {air }}$ based on the inlet velocity, $U_{0}$, kinematic viscosity of air, $\nu_{a i r}$, and the half of the major axis dimension, $a / 2$, of the ellipse. The major axis dimension of the ellipse, $a$, and the air velocity, $U_{0}$, were set to $0.6 \mathrm{~m}$ and $5 \mathrm{~m} / \mathrm{s}$, respectively, so that the Reynolds number is c.a. $1.0 \times 10^{5}$. A constant time step, $\Delta t$ of $6.0 \times 10^{-3} \mathrm{~s}$ was used in the simulations. To match the boundary conditions, at the inlet and outlet of the solution domain, velocity boundary conditions satisfying the mass conservation were imposed. This led to an inlet velocity, $U_{0}$, of $5 \mathrm{~m} / \mathrm{s}$. The free-stream turbulence intensity was taken as $10 \%$ of the inlet velocity, $U_{0}$. At the inlet and outlet, turbulent kinetic energy, $k$, and dissipation rate, $\epsilon$ were calculated 
from $k=0.005 U_{0}^{2}$ and $\epsilon=k \frac{3}{2} / 0.15 a$, respectively. For the SST $k-\omega$ model computations, specific dissipation rate, $\omega$, was calculated from $\epsilon / k C_{\mu}$, where $C_{\mu}$ is an empirical constant equal to 0.09. No slip boundary conditions were used for all of the walls. For other cases $\left(R e=1.0 \times 10^{4}\right.$ and $\left.R e=1.0 \times 10^{3}\right)$, the inlet velocity was adjusted to attain the desired Reynolds number. In an attempt to repeat Dunnett's calculations [4] first, simulations with the standard $k-\epsilon$ turbulence model were run on grids with different resolutions, and grid convergence has been investigated at this Reynolds number $\left(1.0 \times 10^{5}\right)$. Then, the same calculations were performed on the same grids with an improved type of $k-\epsilon$ family of turbulence model, namely, RNG $k-\epsilon$ model, and separately with SST $k-\omega$ turbulence model. Although successively finer grids were used for grid convergence analysis, focus was placed principally on the level of grid resolution in the near-wall region because the location of separation and, therefore, the size of the recirculation zone, are affected mainly by the grid resolution in this region.

On the whole, seven different grids becoming denser around the ellipse were generated using a commercial grid generation software, called GAMBIT. Twodimensional structured boundary layer grids extending c.a. 10-15\% of the major axis of the ellipse were generated to control the spacing of the first interior grid point away from the ellipse $\left(\Delta y_{1}\right)$. The grid for the rest of the domain was created using Quad/Pave scheme, which leads to non-orthogonal rectangular cells. The consequent grid structure around the ellipse is shown in Figure 3.2. On the whole, the grids generated differ in number of total cells as well as in spacing of the first interior grid $\left(\Delta y_{1}\right)$. The details of the grids used are listed in Table 3.1

The commercial CFD software FLUENT 6.3 was used to solve two/threedimensional, Navier-Stokes and URANS equations with the Finite Volume Method (FVM) on a collocated grid. The Quadratic Upwind Interpolation (QUICK) scheme was used for spatial discretization of convective terms in the momentum transport equation, whereas the second-order upwind scheme was 


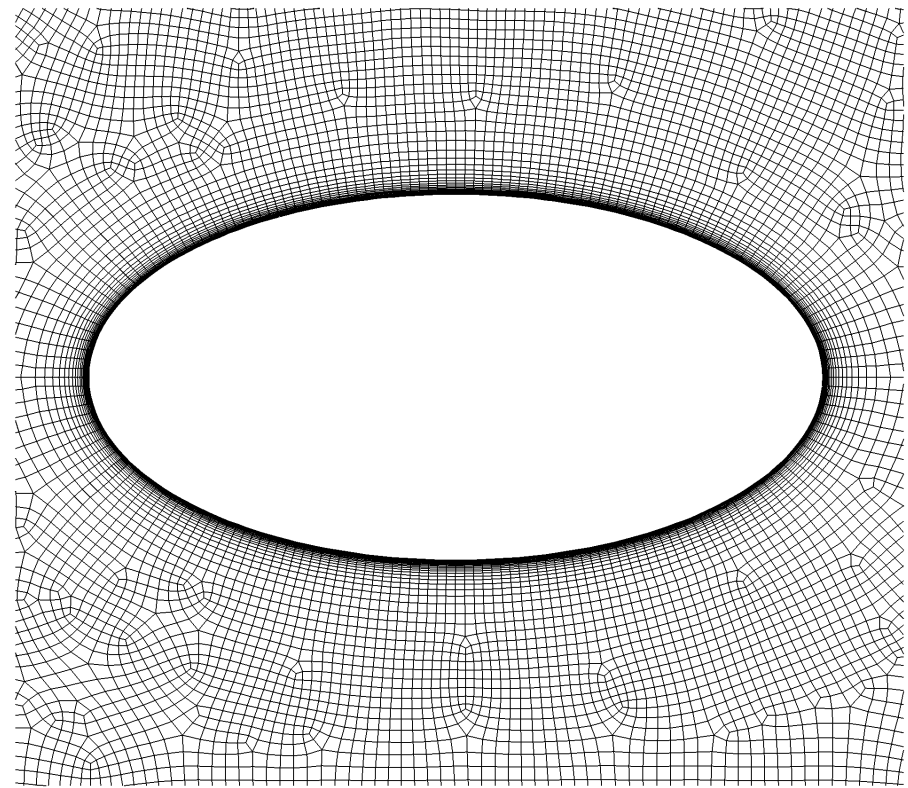

Figure 3.2: Detail view of grid (G6) around the ellipse

used for discretization of all other convective terms in other transport equations. All diffusive terms were discretized by second order central differencing scheme. A first-order-accurate unconditionally stable implicit scheme was used for time discretization. SIMPLEC algorithm was used for velocity-pressure coupling. Sufficient convergence at each time step was assumed to be reached when the sum of the scaled residuals was less than $1.0 \times 10^{-4}$. In cases where the flow exhibited unsteady behavior, simulations were run until a nearly periodic flow was observed to analyze the frequency response of the flow.

Table 3.1: Number of Cells Used

\begin{tabular}{llll}
\hline Grid & $R e=1.0 \times 10^{5}$ & $R e=1.0 \times 10^{4}$ & $R e=1.0 \times 10^{3}$ \\
\hline G1 & $9,594\left(\Delta y_{1}=7.2 \times 10^{-3} \mathrm{~m}\right)$ & $51,144^{B}\left(\Delta y_{1}=4.6 \times 10^{-4} \mathrm{~m}\right)$ & $51,144^{B}\left(\Delta y_{1}=4.6 \times 10^{-4} \mathrm{~m}\right)$ \\
G2 & $24,993\left(\Delta y_{1}=3.6 \times 10^{-3} \mathrm{~m}\right)$ & & \\
G3 & $49,037\left(\Delta y_{1}=2.7 \times 10^{-3} \mathrm{~m}\right)$ & & \\
G4 & $59,340^{A}\left(\Delta y_{1}=1.8 \times 10^{-3} \mathrm{~m}\right)$ & & \\
G5 & $76,663^{A}\left(\Delta y_{1}=9.0 \times 10^{-4} \mathrm{~m}\right)$ & & \\
G6 & $82,081^{A}\left(\Delta y_{1}=1.8 \times 10^{-4} \mathrm{~m}\right)$ & & \\
G7 & $93,883^{A}\left(\Delta y_{1}=6.0 \times 10^{-5} \mathrm{~m}\right)$ & & \\
\hline
\end{tabular}

$A$ Enhanced wall treatment was used in $k-\epsilon$ model calculation

$B$ Transitional flow modifications were enabled in SST $k-\omega$ model calculations 
In the calculations, standard wall functions were used if the average $y^{+}$ (first interior grid in wall distance units) is greater than 30; otherwise, the enhanced wall treatment approach was used. The enhanced wall treatment method combines a two-layer model with enhanced wall functions and becomes identical to the two-layer zonal method when $y^{+} \approx 1$. In the present study, this condition is satisfied on the finest grid (G7). On the other hand, provided that the grid resolution is high enough, $\left(y^{+} \approx 1\right)$ SST $k-\omega$ model does not need any wall functions in the near-wall regions and, therefore, is integrated down to the wall without using any damping functions. The effect of grid refinement on prediction of the angle of separation, $\theta_{s}$ (location where axial wall shear stress is zero on the surface of the ellipse), as measured clockwise from the upstream stagnation point, and the length of recirculation zone, $X_{r}$ (distance between the location of confluence point and the downstream stagnation point of the ellipse) is illustrated in Figure 3.3. From Figure 3.3a., it can be seen that, in general, the angle of separation decreases (location of separation moves upstream) with increasing grid resolution in the near-field. Interestingly, for large values of $\Delta y_{1}$ (from $1.8 \times 10^{-3}$ to $7.2 \times 10^{-3} \mathrm{~m}$ ) RNG $k-\epsilon$ and SST $k-\omega$ model predictions collapse on the same curve. However, the same agreement is not seen in the convergence of the recirculation zone length (Figure 3.3b). It is important keep in mind that the results are influenced by the near-wall modeling too. Table 3.2 lists the laminar and turbulent viscosities along with their ratios at a point in the near-wall region of the ellipse. The listed values are from the RNG $k-\epsilon$ model simulations on two different grids with different near-wall treatments. From the table one can see that on grid G3 where the standard wall function was employed, the turbulent viscosity dominates the laminar viscosity. However, it is well known that very close to the wall there is a viscous sublayer where the laminar viscosity takes over. The very low value of ratio of $\mu_{T} / \mu_{L}$ indicates that this phenomena was captured in the solution on grid G7. It should also be noted at this point that the numerical viscosity may become important. On the whole, the solution on grid G3 seems relatively less accurate due to combined effect of wall function and its restrictions on the grid resolution. 


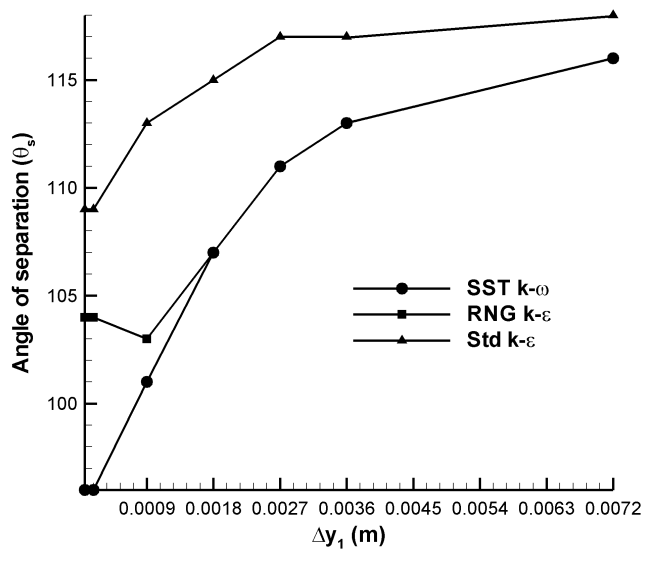

a)

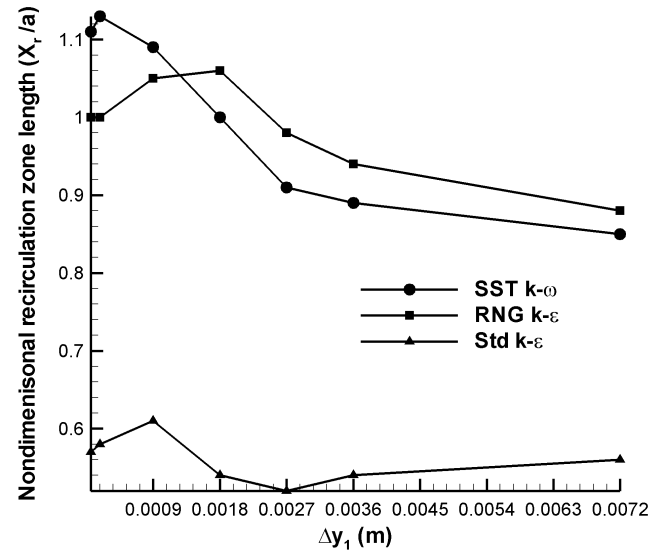

b)

Figure 3.3: Variation of a) angle of separation, b) nondimensional recirculation zone length $(a=0.6 \mathrm{~m})$, with $\Delta y_{1}$

Table 3.2: Laminar and turbulent viscosities in the near-wall region $(x=2.146 a$, $y=6.427 a)$

\begin{tabular}{lccc}
\hline Grid & $\mu_{L}(\mathrm{~kg} / \mathrm{m} \mathrm{s})$ & $\mu_{T}(\mathrm{~kg} / \mathrm{m} \mathrm{s})$ & $\mu_{T} / \mu_{L}$ \\
\hline $\mathrm{G} 3\left(y^{+}=45\right)$ & $1.7894 \times 10^{-5}$ & $1.1102 \times 10^{-4}$ & 6.2 \\
$\mathrm{G} 7\left(y^{+} \approx 1\right)$ & $1.7894 \times 10^{-5}$ & $6.6088 \times 10^{-7}$ & 0.03 \\
\hline
\end{tabular}

Assuming the most reliable solutions are obtained on a fully resolved grid $\left(y^{+} \approx 1\right)$, the behaviors of the solutions at moderate and low Reynolds number $\left(1.0 \times 10^{4}\right.$ and $1.0 \times 10^{3}$, respectively $)$ were tested on a grid fine enough to resolve the near-wall region.

For the high Reynolds number case $\left(\mathrm{Re}=1.0 \times 10^{5}\right)$, the streamtraces in the near-field of the ellipse predicted by the standard $k-\epsilon$ turbulence model calculations on the coarsest grid (G1) are shown in Figure 3.4, where it is clear that even with the standard wall functions, the standard $k-\epsilon$ turbulence model captures boundary layer separation leading to a steady and symmetric recirculation zone in the wake of the ellipse. For the same conditions, these vortex pairs developed in the wake were not captured in Dunnett's [4] simulations. The possible reasons for this disagreement might be the differences in quality and resolution of the grid around the ellipse or implementation of the 


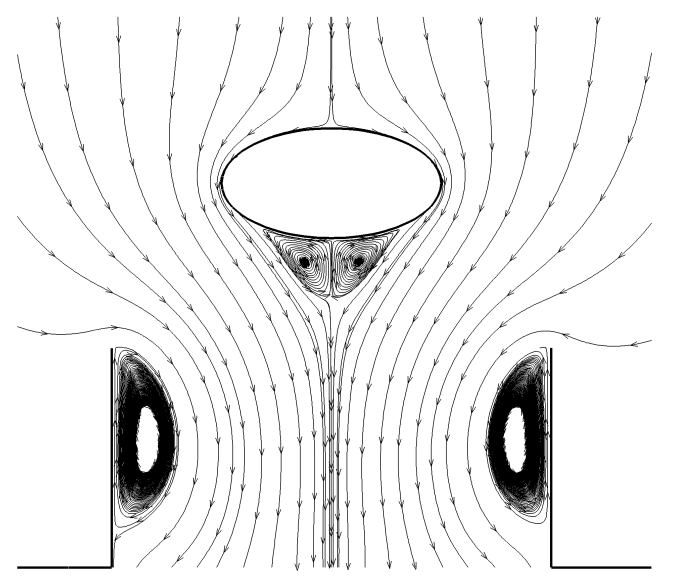

Figure 3.4: Streamtraces predicted on coarse grid (G1) with the standard $k-\epsilon$ model at $\mathrm{Re}=1.0 \times 10^{5}$

turbulence model. The recirculation zones attached to the hood walls are due to separation of the incoming flow, especially from the sides, at the hood walls. These recirculation zones can also be seen in Dunnett's predictions.

From Figure 3.3, shown in the description of two-dimensional case, it is seen that the length of the recirculation zone is correlated with the location of separation point, as $\theta_{s}$ decreases toward $90^{\circ}, X_{r}$ increases, and vice versa. Furthermore, the predicted $X_{r}$ values using the standard $k-\epsilon$ model computations are remarkably smaller compared to those predicted by RNG $k-\epsilon$ and $\operatorname{SST} k-\omega$ models. This situation may be attributed, in general, to a shortcoming of the standard $k-\epsilon$ models, whereby they produce high levels of turbulent viscosity, and hence, tends to suppress the vortices. This issue is examined further for moderate Reynolds number $\left(1.0 \times 10^{4}\right)$ in more detail.

Figure 3.5 depicts to what extent the predicted recirculation zones extend toward the hood. It is observed that for the condition under consideration, the recirculation zone does not extend into the hood. If it extended into the hood, in practice, the contaminants might be carried to the breathing zone of the worker by the reverse flow. The recirculation zones predicted by both turbulence models are smaller than in cases without a hood (not shown here). The convergence 


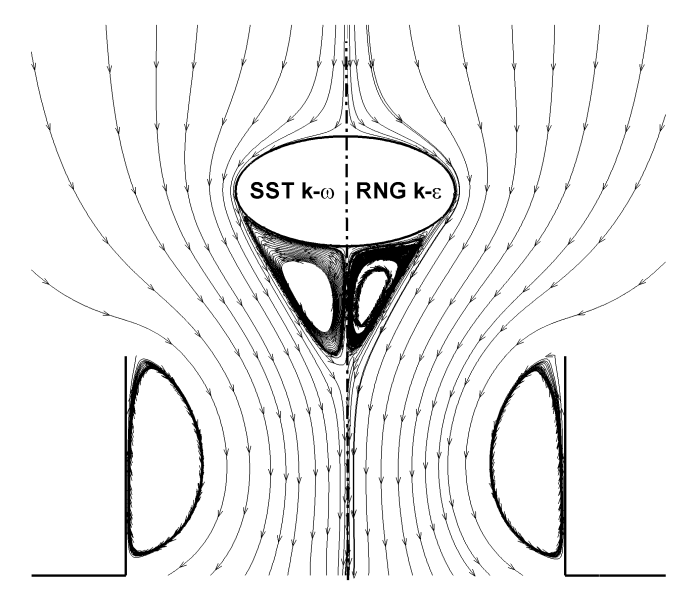

Figure 3.5: Comparison of the streamtraces predicted by fully resolved (G7) $\mathrm{SST} k-\omega$ and RNG $k-\epsilon$ models at $\mathrm{Re}=1.0 \times 10^{5}$

of the airflow into the hood suppresses the recirculation region and makes it narrower toward the hood entrance.

Figure 3.6 illustrates how the SST $k-\omega$ model predicts drastically different flow patterns compared with the $k-\epsilon$ models at a moderate Reynolds number $\left(1.0 \times 10^{4}\right)$. Theoretically, at this Reynolds number, vortex shedding should be observed for a flow around an ellipse immersed in a free-stream [6]. However, the presence of hood (contraction) and, hence, the acceleration of the flow into the hood are expected to change the flow pattern downstream of the ellipse. From the SST $k-\omega$ model predictions shown in Figure 3.6a, the anticipated unsteadiness and asymmetry of the flow due to onset of vortex shedding can be clearly observed. However, the vortex shedding is suppressed due to convergence of the flow into the hood, and a pair of vortices attached to the ellipse undulates instead.

On the other hand, the standard and RNG $k-\epsilon$ models, still predicted steady recirculation zones, as shown in Figures $3.6 \mathrm{~b}$ and 3.6c. This result not only confirms the point made by Franke and Rodi [51] on the failure of standard $k-\epsilon$ turbulence model in predicting unsteady separated flows behind bluff bodies, but also extends it to RNG $k-\epsilon$ model. In an attempt to understand why 


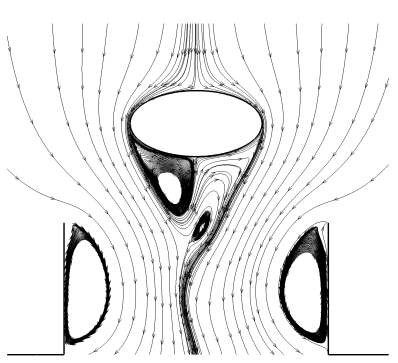

a)

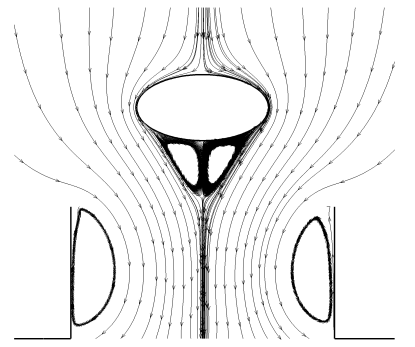

b)

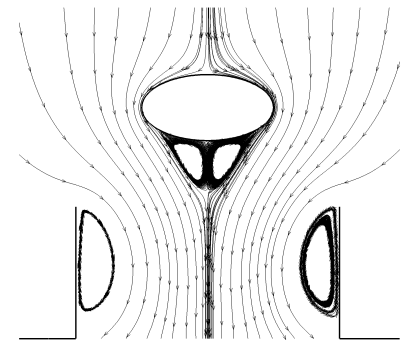

c)

Figure 3.6: Predicted streamtraces at 4000 time step at $\mathrm{Re}=1.0 \times 10^{4}$, a) SST $k-\omega$ model, b) RNG $k-\epsilon$ model, c) standard $k-\epsilon$ model

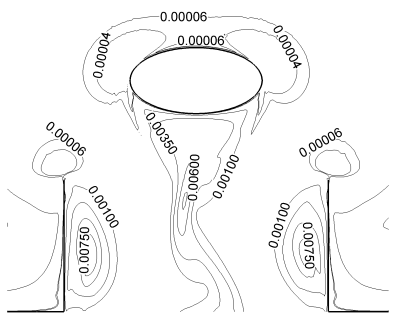

a)

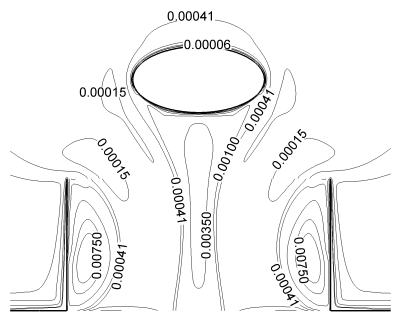

b)

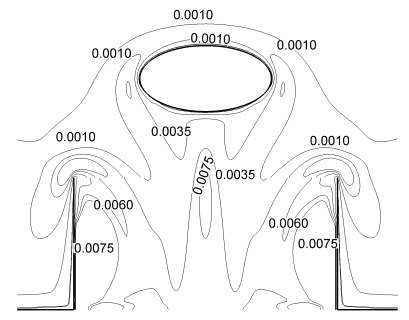

c)

Figure 3.7: Predicted turbulent viscosities at 4000 time step at $\operatorname{Re}=1.0 \times 10^{4}$, a) SST $k-\omega$ model, b) RNG $k-\epsilon$ model, c) standard $k-\epsilon$ model

the $k-\epsilon$ models fails to capture the unsteadiness, contours of the turbulent viscosities $\left(\mu_{T}\right)$ computed by all models are plotted in Figure 3.7. A closer look in proximity of the ellipse reveals that the standard and RNG $k-\epsilon$ turbulence models lead to higher turbulent viscosities in this region, which, in turn, change the effective Reynolds number that can be defined as $R e_{\text {eff }}=D U \rho / \mu_{\text {eff }}$, where $\mu_{e f f}$ is the summation of laminar and turbulent viscosities $\left(\mu_{e f f}=\mu_{L}+\mu_{T}\right)$. An increase in the effective viscosity $\left(\mu_{e f f}\right)$ gives rise to a decrease in $R e_{e f f}$ and, hence, to a change in flow regime. Reducing the grid size substantially decreases the turbulent viscosity only slightly, which does not improve the predictions much. Assuming that the SST $k-\omega$ model predictions are more accurate, it can be concluded that the standard $k-\epsilon$ turbulence model damps out the velocity variations around the ellipse, leading to a high degree of smoothing of the gradients in the flow. 
The time history of the streamwise velocity, from the SST $k-\omega$ model computation, monitored at a point $(x=2.4 a, y=6.57 a)$ in the wake, is presented in Figure 3.8a. The calculated power spectrum of the presented velocity oscillations is illustrated in Figure 3.8b. As can be seen from the figure, the frequency of the oscillations is $1.91 \mathrm{~Hz}$, which corresponds to a Strouhal number $(S r)$ of 2.29 based on the dimension of the major axis of the ellipse $(a=0.6 \mathrm{~m})$ and the free-stream velocity $\left(U_{0}=0.5 \mathrm{~m} / \mathrm{s}\right)$. These high frequency $(S r=2.29)$ oscillations are due mainly to the effect of contraction that causes the flow converge into the hood, leading to an alternating confinement of the vortices from either side, immediately downstream of the ellipse. Therefore, the vortices do not have enough time to shed freely, as it happens in the absence of hood. To ensure that this high Strouhal number is not due to the mesh resolutions, simulations in the absence of contraction were run on the same mesh, and a Strouhal number of 0.25 was obtained, which deviates slightly from the theoretical value of c.a. 0.2 , reported for flows past an ellipse immersed in a freestream [6]. The blockage by the side walls present in the computational domain might have contributed to this slight deviation from the theoretical value. At low Reynolds number $\left(1.0 \times 10^{3}\right)$, both laminar and SST $k-\omega$ model computations were performed. Theoretically, the asymmetry in the wake flow is expected to be more pronounced in the low Reynolds number case. The much stronger oscillations obtained from the laminar computations (Figures 3.9a and 3.9b) confirm this hypothesis. The dominant frequency $(0.086 \mathrm{~Hz})$ of the velocity oscillations corresponds to a Strouhal number of 1.03.

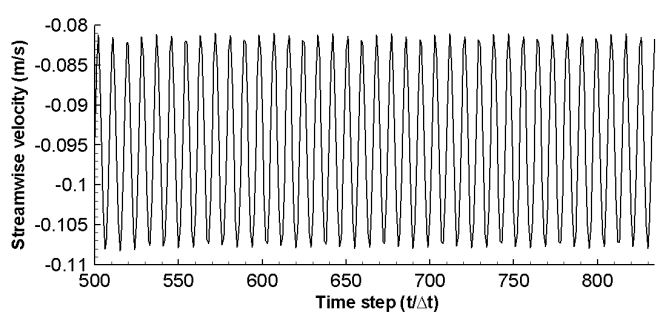

a)

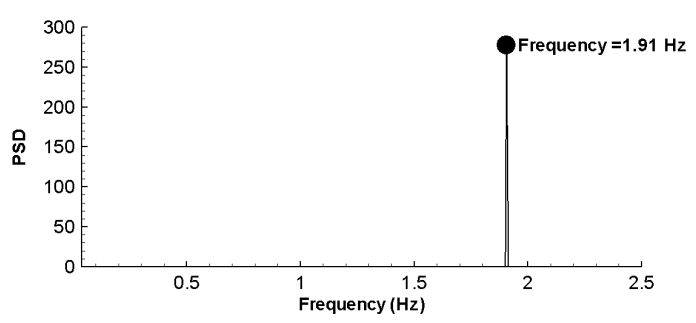

b)

Figure 3.8: Streamwise velocity from SST $k-\omega$ model at a point $(x=2.4 a, y=$ $6.57 a$ ) at $\operatorname{Re}=1.0 \times 10^{4}$, a) time history, b) power spectral density (PSD) 


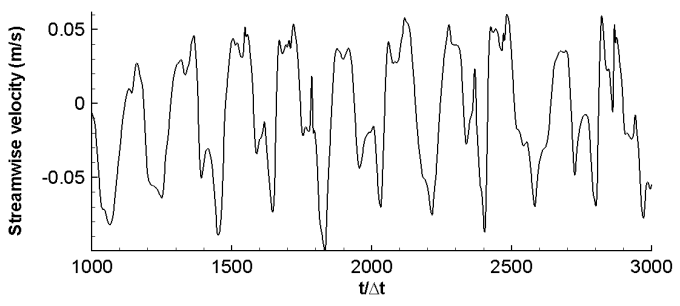

a)

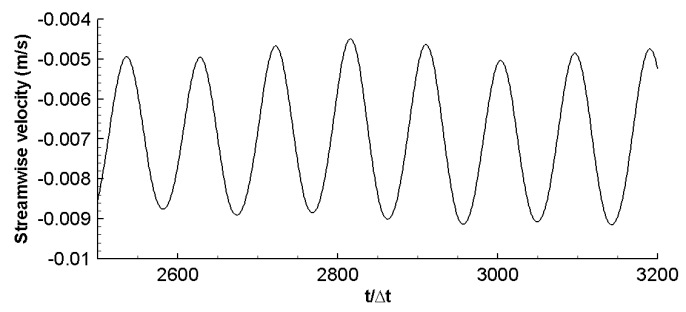

c)

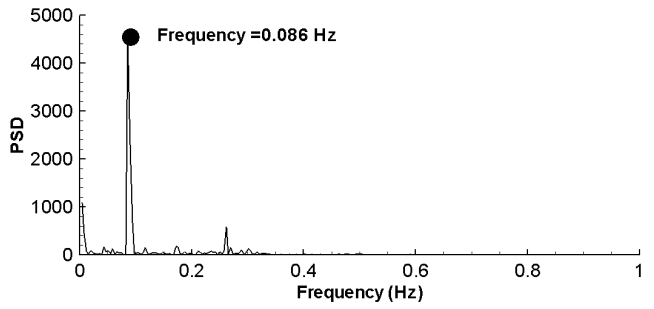

b)

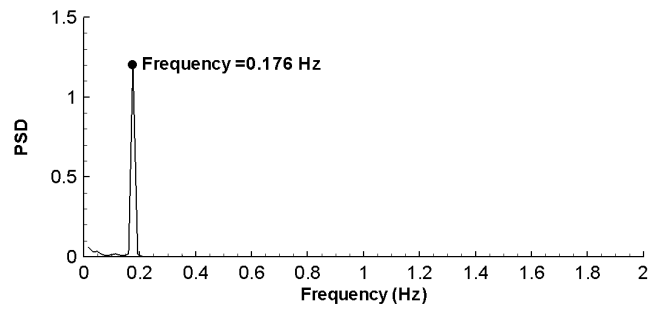

d)

Figure 3.9: Time history of streamwise velocity and corresponding PSD spectrum at a point $(x=2.4 a, y=6.57 a)$ at $\operatorname{Re}=1.0 \times 10^{3}$, a-b) Laminar, c-d) SST $k-\omega$ model

On the other hand, the SST $k-\omega$ model, which is considered to be suitable for low Reynolds number flows as well, captured the oscillatory behavior with a frequency of $0.176 \mathrm{~Hz}(S r=2.11)$ (Figures 3.9c and 3.9d). Although these oscillations are much weaker compared with the ones from laminar computations, they point to the fact that SST $k-\omega$ turbulence model can be used for transitional flows. These unsteady flow structures captured in the present laminar calculations are much more pronounced than those reported by Dunnett [4] for the case of $\operatorname{Re}=1.0 \times 10^{3}$.

On the whole, the predictions indicated that depending on the suction rate by the hood, steady or unsteady recirculation zones can form in the wake of the worker. Because of the effect of converging flow, the sizes of these zones are limited to the spacing between the worker and the hood; so, for the given flow configuration, the reverse flow is not expected to carry the contaminants from the hood back to the breathing zone of the worker. However, it should be kept in mind that contaminants can leak from the hood or move toward the worker by the mechanism of turbulent diffusion. At low suction rates (low Re), the 
unsteadiness in the flow can have an enhancing effect on the dispersion of the contaminants inside the hood that in turn may increase the exposure level. The flow patterns can change significantly if the worker stands closer to the hood face. 


\section{Chapter 4}

\section{Experiments and Numerical Simulations}

\subsection{Experimental Setup}

Smoke tests are usually performed to visualize airflow patterns inside enclosing hoods and to qualitatively evaluate containment of enclosing hoods. In the present study, flow visualization experiments were performed in conjunction with the CFD simulations to obtain qualitative information about the airflow. The experiments were performed in the Ventilation Wind Tunnel of West Virginia University, which is schematically depicted in Figure 4.1. The wind tunnel offers a working area of $4.92 \mathrm{~m} \times 2.74 \mathrm{~m} \times 3.66 \mathrm{~m}$ (Length $\times$ Height $\times$ Width). Although it is relatively short compared to its height and width, the uniformity of the velocity distribution is considered to be acceptable for the purposes of this study.

As shown in Figure 4.1, in the wind tunnel, a manikin to simulate the worker and a bench-top enclosing hood are positioned perpendicular to the cross-draft. The manikin is located near the center of the width of the wind tunnel. The dimensions of the enclosing hood are $0.91 \mathrm{~m} \times 0.76 \mathrm{~m} \times 1.14 \mathrm{~m}$ (Width $\times$ Height $\times$ Depth). The top and right wall of the enclosing hood is made of Plexiglass ${ }^{\mathrm{TM}}$ to facilitate visualization experiments. The hood has a plenum section with a 95\% opaque perforated plate to adjust the pressure distribution for a more uniform flow field at the hood face (entry plane). Also it has a $45^{\circ}$ tapered 


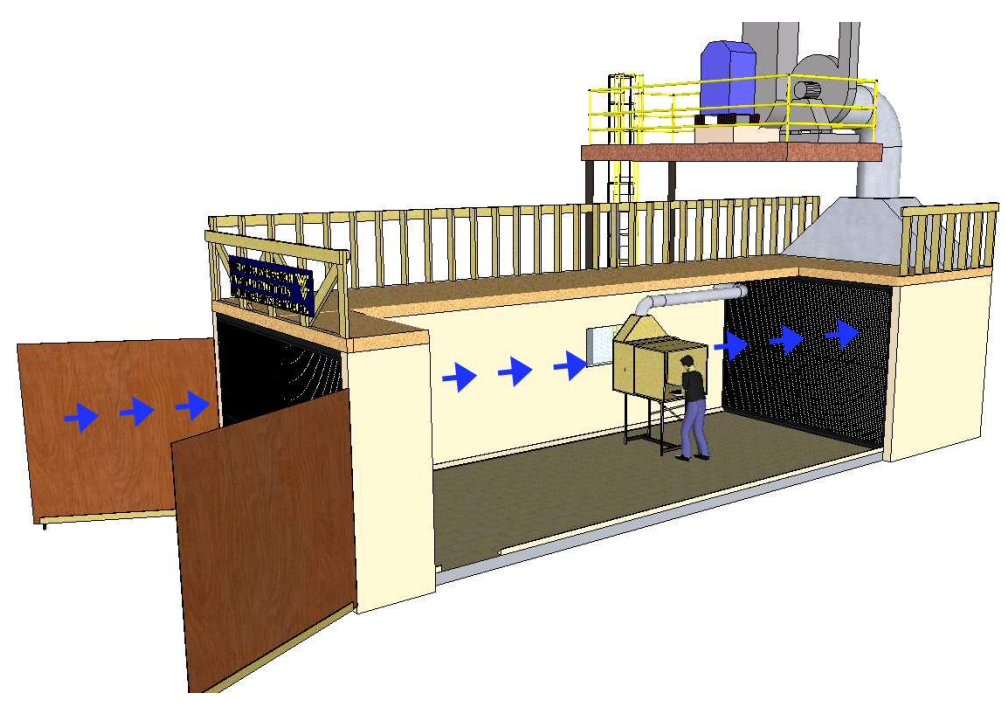

Figure 4.1: Schematic representation of the wind tunnel and its contents. Blue arrows indicate direction of cross-draft. (Courtesy of Dr. S.Guffey)

takeoff with typical rectangular to round transition. The hood fan draws air through a circular duct connected to the takeoff. The manikin, placed in front of the hood with the center line on the symmetry plane, is 66 inches $(1.68 \mathrm{~m})$ high, anthropometrically-scaled (including realistic facial features and short hair) male with hollow cavities in the head, torso, legs and arms. The manikin is clothed with loose-fitting pants and tucked-in t-shirt and is standing about 1 inch $(0.025 \mathrm{~m})$ away from the hood face. However, the head of the manikin remains partially inside the hood, to simulate a realistic condition. Since the size and shape of the human body can vary greatly from worker to worker, for the sake of brevity a simple body approximating the anthropometrically scaled one is also tested. The simple manikin consists of a sphere, an elliptical cylinder (aspect ratio $=0.5$ ) and two circular cylinders, representing the head, torso and legs, respectively. Figure 4.2 shows the manikins employed in this study.

In the flow visualization experiments, a tracer smoke was released from the upstream of the hood and manikin, so that it flowed with the cross-draft and was drawn into the hood due to suction force created by the hood fan. In some cases, the smoke was introduced from various locations around the manikin and the hood in order to visualize localized phenomena. The transport of smoke pulled by the hood was recorded using a high definition video camera for reviewing. 


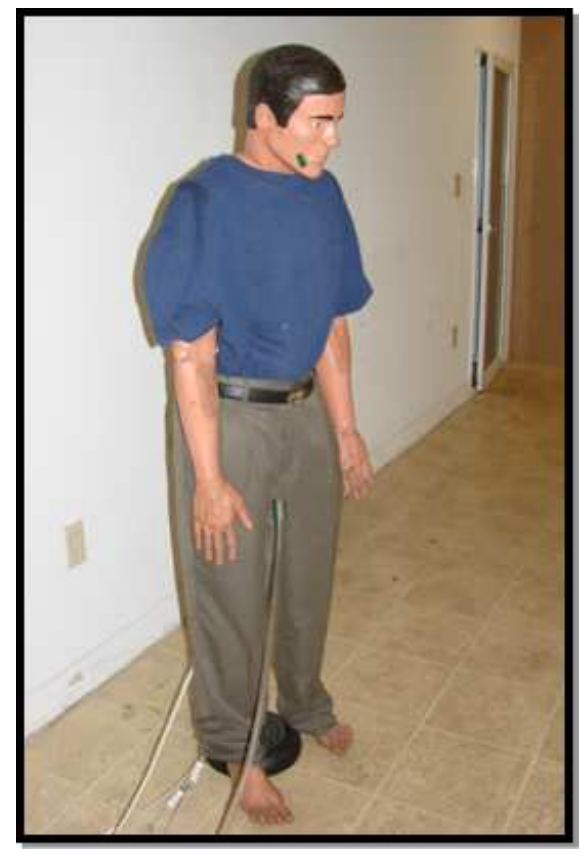

a)

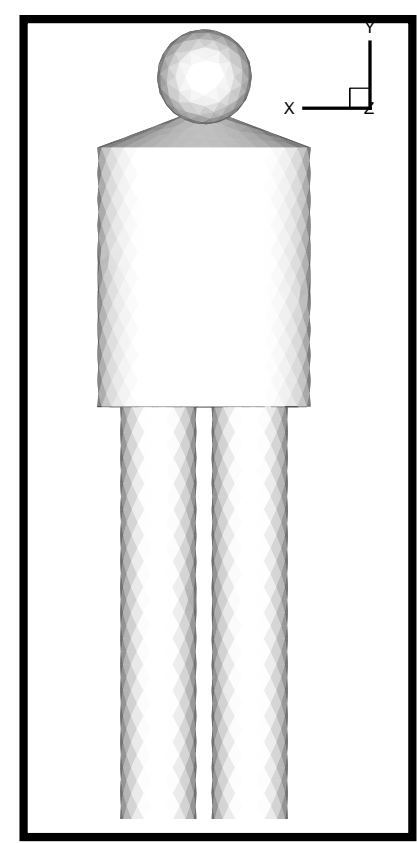

b)

Figure 4.2: Manikins used in the experiments a) anthropometrically scaled, b) simple

The flow patterns captured were qualitatively compared with those predicted by the simulations.

It should be mentioned that, the present flow visualization experiments were explicitly performed by the author of this study using the experimental set-up of Dr. S. Guffey of the WVU Industrial and Management Systems Engineering Department. On the other hand, the exposure experiments, in which a contaminant, namely Freon 134a, is released from a round source placed on the working surface of the hood within the arm length of the worker, were conducted by Guffey and his co-workers for various conditions [52]. Further details about the experimental set-up can be found in [53]. Here, the experimental data provided by Guffey et al. [52] is used to compare predicted concentration levels in the breathing zone of the worker. The experimental results are presented along with the numerical ones in the following sections. 


\subsection{Numerical Simulations}

CFD simulations were performed to investigate the flow patterns and contaminant dispersion inside the enclosing hood. For this purpose, a computational domain representing the experimental configuration was generated using a commercial mesh generation software, called GAMBIT. Except for the length of the wind tunnel, the geometry matches the experimental configuration as closely as possible. The length of the computational domain was kept intentionally shorter than the actual length of the wind tunnel to prevent the artificial decay of turbulence, generated at the inlet. The computational domain begins approximately 5 shoulder diameters $\left(D_{s} \approx 0.45 \mathrm{~m}\right)$ upstream of the hood.

Due to excessive computational time associated with the anthropometrically scaled manikin, the majority of the simulations were performed using the simple manikin. Simulations with the simple manikin were performed on three different, non-uniform, unstructured meshes consisting of 518,101 (coarse mesh), 1,059,013 (medium mesh), 1,181,922 (fine mesh) tetrahedral cells in order to assess the sensitivity of the numerical solutions to the mesh resolution. In all cases, finer cells have been employed around the manikin and inside the hood to resolve the flow in this area better. The main difference between the three meshes is the cell density around the manikin. Figure 4.3 details the mesh structure in the region of interest.

The simulations were performed for the set of cross-draft and face velocity combinations given in Table 4.1. The table also lists a set of letters assigned to each Case to be used henceforth. Based on the shoulder diameter of the manikin and the approaching velocity of the air at $297 \mathrm{~K}$, the Reynolds number is in the range of about 4,500-13,700. Both isothermal and non-isothermal simulations were conducted to investigate the flow dynamics, exposure levels and thermal impact of worker's body on the exposure level. Gravitational force is always included in the simulations. 


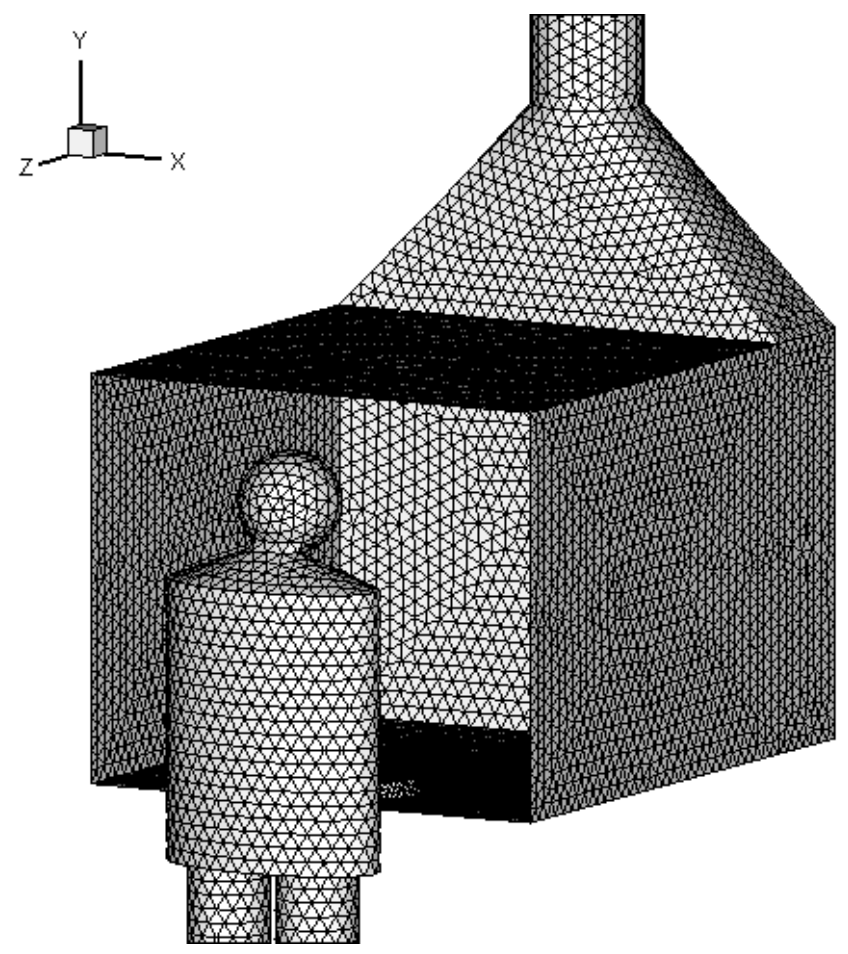

Figure 4.3: Detail view of coarse mesh

Three-dimensional URANS calculations were carried out using RNG $k-\epsilon$ turbulence model [54]. The contaminant was treated as a passive scalar. Therefore, along with the URANS equations, the generic passive scalar transport equation (Equation 2.3) was also solved. The commercial CFD software, FLUENT 6.3, was used to solve the governing equations with the Finite Volume Method (FVM) on co-located grids. The MUSCL scheme has been used for spatial discretization of convective terms appearing in passive scalar transport equation, whereas second order upwind scheme has been used for discretization of all other convective terms in other transport equations. All diffusive terms

Table 4.1: Air velocities used in the simulations

\begin{tabular}{cccc}
\hline Case & $V_{\text {Cross-draft }}(\mathrm{m} / \mathrm{s})$ & $V_{\text {Face }}(\mathrm{m} / \mathrm{s})$ & $V_{\text {Cross-draft }} / V_{\text {Face }}$ \\
\hline $\mathrm{A}$ & 0.073 & 0.999 & 0.073 \\
$\mathrm{~B}$ & 0.073 & 0.852 & 0.086 \\
$\mathrm{C}$ & 0.073 & 0.691 & 0.106 \\
$\mathrm{D}$ & 0.073 & 0.550 & 0.133 \\
$\mathrm{E}$ & 0.182 & 0.981 & 0.186 \\
$\mathrm{~F}$ & 0.291 & 0.969 & 0.300 \\
$\mathrm{G}$ & 0.291 & 0.526 & 0.553 \\
\hline
\end{tabular}


were discretized by the second order central differencing scheme. A first-orderaccurate implicit scheme was used for time discretization with a time step of $1 \times 10^{-3}$ s. SIMPLEC algorithm was used for velocity-pressure coupling. Coarse grid simulations were first run at steady state with a larger convergence criterion $\left(10^{-3}-10^{-4}\right)$ in residuals. The converged results were then used as initial condition for the unsteady simulations which were run for several flowthrough-times until periodicity in the flow was observed. Medium and fine grid simulations were started from interpolated coarse and medium grid solutions, respectively. Again, simulations were run for several flow-through-times until a periodicity in the flow was observed. Sufficient convergence at each time step was assumed to be reached when the sum of the normalized absolute residuals is less than $10^{-6}$ for energy and $10^{-4}$ for other variables.

The system under consideration has one inlet (wind tunnel inlet) and two outlets (wind tunnel and hood outlets) for the flow. As for the boundary conditions, fixed velocity boundary conditions, specifying outward flow, were imposed at the outlets. On the other hand, a constant pressure (1atm) boundary condition was specified at the wind tunnel inlet. For all of the inlet and outlet boundary conditions, $10 \%$ turbulent intensity and turbulent viscosity ratio of 10 were assumed. No-slip boundary conditions were imposed at all solid walls, such as the wind tunnel walls, hood walls and manikin. The effects of the walls were computed by the standard wall-function. The typical $y^{+}$values change in the range of 60-250. The passive scalar (contaminant; Freon 134b), 10\% by volume fraction of scalar in air, was released from the top surface of the round source at a total mass flow rate of $1.34 \times 10^{-5} \mathrm{~kg} / \mathrm{s}$. At the top of the source a turbulent intensity of $10 \%$ and turbulent viscosity ratio of 10 were specified. The plenum section was modeled by a porous jump boundary condition. 


\subsubsection{Isothermal Flow}

In this part of the study, the flow was limited to isothermal condition, i.e. the effect of worker's body heat was not included in the calculations. The air, the manikin, and the walls were at the same temperature (297K). The simulations were run using RNG $k-\epsilon$ turbulence model on three grids, the details of which have been introduced previously. The simulated concentrations were monitored at three points as shown in Figure 4.4a. Among these points, Point 1 and Point 2 correspond to the location of sampling ports adjacent to the nose and mouth of the worker, respectively (see Figure 4.4b). The reason for the extra point; Point 3 , is solely to check how the flow and concentrations on both sides of the worker's line of symmetry compares. It is of interest to note that while in the experiments the sampling was accomplished by sucking the contaminated air at a low rate over $15 \mathrm{~min}$, in the simulations the vertex averaged values were monitored at exactly Point 1, 2 and 3 which were $1 \mathrm{~cm}$ off the surface of manikin (worker). To this end, experimental measurements appear to have better temporal and spatial averaging compared to the simulations. The reason for selecting points $1 \mathrm{~cm}$ off the manikin's surface was to prevent any possible errors that might arise from selecting monitoring points on the manikin's the surface, where a boundary condition for the passive scalar was prescribed, while maintaining a reasonable proximity to the locations of the sampling ports used in the experiments.

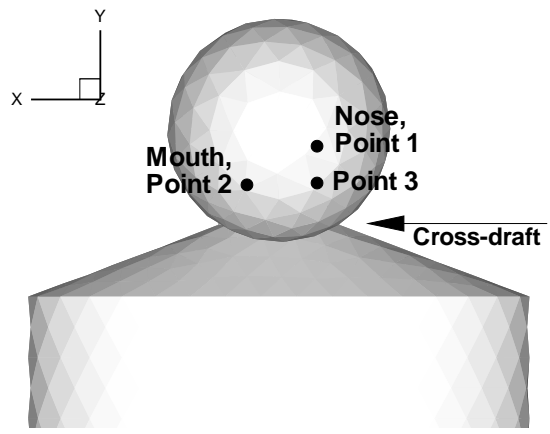

a)

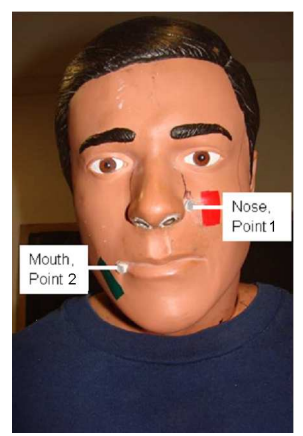

b)

Figure 4.4: Location of data monitoring/sampling points (Mouth; Point 1: $x=2.03 \mathrm{~m}, y=1.45 \mathrm{~m}, z=1.83 \mathrm{~m}$, Nose; Point $2: x=2.09 \mathrm{~m}, y=1.42 \mathrm{~m}, z=1.84 \mathrm{~m}$, Point 3: $x=2.09 \mathrm{~m}, y=1.42 \mathrm{~m}, z=1.84 \mathrm{~m}$ ) a) simple manikin, b) complex manikin 
In an attempt to test the grid sensitivity of predicted exposure trends, the nondimensionalized long-time-averaged concentrations computed on all three grids are compared with each other at Point 1 and Point 2 in Figure 4.5. Note that for the convenience of comparison, experimental measurements obtained using the simple, round manikin are also presented in the figure. Since no attempt was made to match the experimental and the simulated concentrations, the focus is placed more on trend analysis rather than quantitative analysis. At a first glance, it is seen that both the simulated and measured concentrations first increase with increasing $V_{\text {Cross-draft }}$ to $V_{\text {Face }}$ ratio. Then they drop to small values as the ratio increases further. However, a closer look at the figure reveals that the coarse grid computations could not capture the same trend as the experiments. Clearly, the location of the peak does not match with the one found in the experiments, especially in Figure 4.5a. With increasing grid resolution, however, the curve shifts to right and the location of the peak appraoches the same location found from the exposure experiments. This behavior indicates that a different flow regime was predicted on the coarse grid. Indeed, this is clearly seen from Figure 4.6 where the long-time averaged flow and concentration fields calculated on the coarse and fine grids are presented. The nonlinear interaction of grid size and quality, turbulence model and numerical scheme seems to lead to flow regime changes with significantly different average flow field in the worker's breathing zone. On the other hand, turbulent Schmidt number $\left(S c_{t}\right)$ is the primary parameter that affects how much the released contaminant diffuses. As mentioned earlier in Chapter 2, where the equations are introduced, the Schmidt number used in this study is 0.7. However, in order to test the sensitivity of the results to this parameter, Case D was simulated with a Schmidt number of 1.0 also. With the increased Schmidt number, the long-time-averaged concentration was found to decrease about $8 \%$. This indicates that by increasing the turbulent Schmidt number to its maximum value of 1.2, better agreements with the experiments could be obtained, but predictions will still be much higher. To this end, the role of relatively large numerical viscosity should be investigated further. 


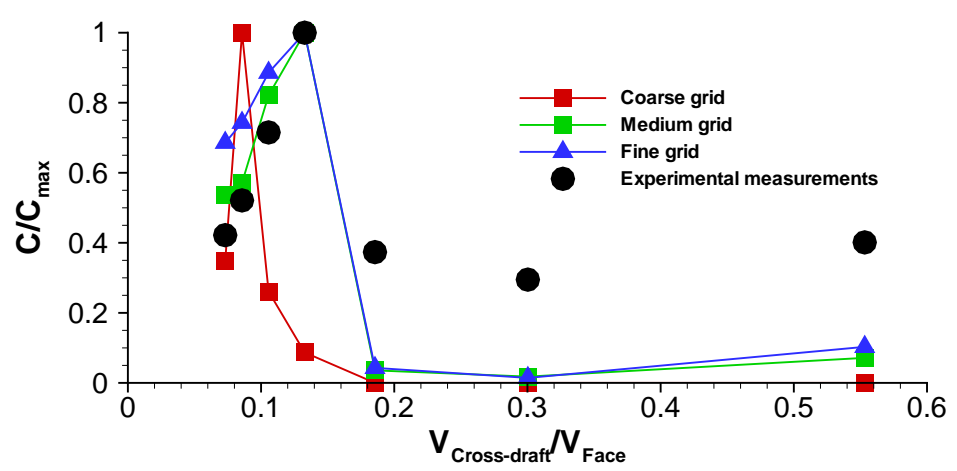

a) $C_{\text {max }}^{\text {coarse }}=60 \mathrm{ppm}, C_{\max }^{\text {medium }}=153 \mathrm{ppm}, C_{\max }^{\text {fine }}=188 \mathrm{ppm}, C_{\max }^{\text {exp }}=19 \mathrm{ppm}$

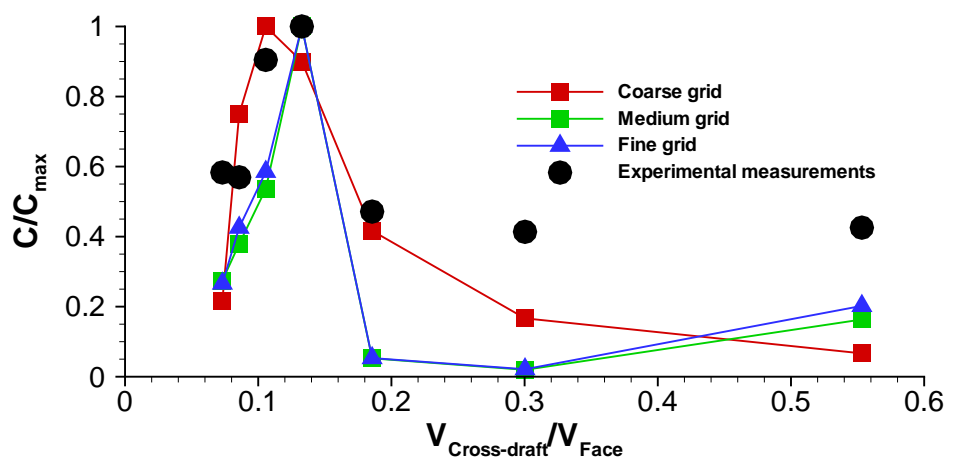

b) $C_{\text {max }}^{\text {coarse }}=23 \mathrm{ppm}, C_{\text {max }}^{\text {medium }}=280 \mathrm{ppm}, C_{\text {max }}^{\text {fine }}=350 \mathrm{ppm}, C_{\max }^{\text {exp }}=24 \mathrm{ppm}$

Figure 4.5: Grid sensitivity of the long-time-averaged concentrations for the simple manikin a) Point 1, b) Point 2

Encouraged by the good agreement observed in Figure 4.5, the fine grid results on planes shown in Figure 4.7 were used in further qualitative analyses. For the sake of brevity, the presented results were limited to Cases A, D, F and G representing low $V_{\text {cross-draft }} /$ high $V_{\text {Face }}$, low $V_{\text {cross-draft }} /$ low $V_{\text {Face}}$, high $V_{\text {cross-draft }} /$ high $V_{\text {Face }}$, high $V_{\text {cross-draft }} /$ low $V_{\text {Face }}$ cases, respectively.

Figure 4.8 illustrates the long-time averaged velocity vector and concentration (passive scalar) fields at the hood face $(x y$-plane-1) for Cases A, D, F and G. It is evident from the velocity vectors that there are vortical motions right in front of the worker. More specifically, there exist two/three vortices at the shoulder level each with the size of about one tenth of the hood height and another large vortex at the waist level. These vortical motions can 


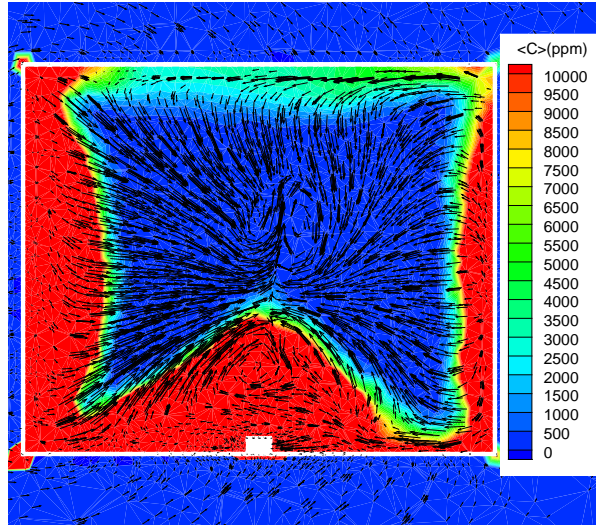

a)

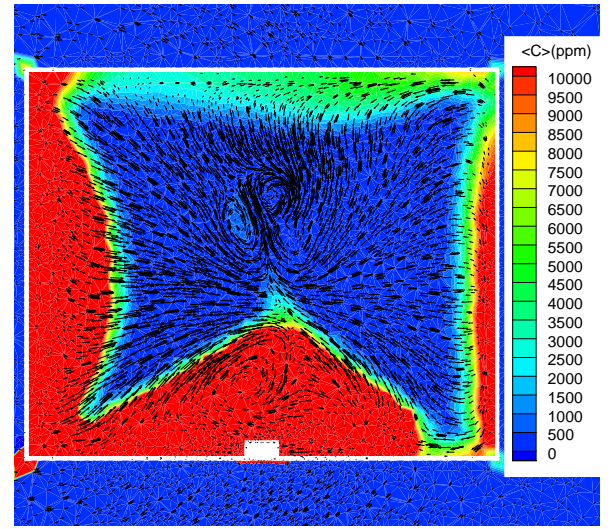

b)

Figure 4.6: Flow and concentration fields at the hood face for Case D a) Coarse grid b) Fine grid

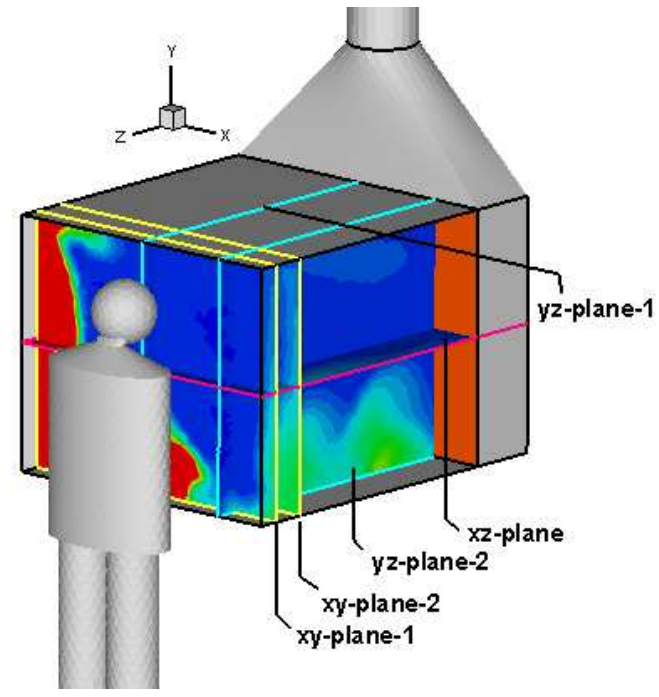

Figure 4.7: Planes on which results are presented; $x y$-plane-1: $z=1.8 \mathrm{~m}$, $x y$-plane-2: $z=1.7 \mathrm{~m}, y z$-plane- $1: \quad x=2.066 \mathrm{~m}, y z-$ plane- $2: \quad x=2.046 \mathrm{~m}, x z-$ plane: $y=1.3 \mathrm{~m}$

also be seen from the three-dimensional stream-traces shown in Figure 4.9, as well as from the snapshot taken during the smoke tests (See Figure 4.10). It is worth noting that the vortical motions at the shoulder level are seen even more clearly in the recorded movies. These small vortices at the shoulder level seemingly arise from the bluff body effect of the worker, whereas separation of the upward flow between the worker and the hood at the edge of the bottom plate 
significantly contributes to the formation of the one at the waist level. Overall, the vortices shown in Figure 4.8 appear to characterize the average flow pattern at the hood face. While this is true, a closer look at Figure 4.8 may reveal that the size and location of the vortices vary from case to case. For example, at high $V_{\text {cross-draft }}$ and low $V_{\text {Face }}$ (Figure 4.8, Case $\mathrm{G}$ and $\mathrm{F}$ ) the smaller vortices are seen to be slightly displaced to the right under the effect of the cross-draft. On the other hand, at low $V_{\text {cross-draft }}$ and high $V_{\text {Face }}$ (Figure 4.8, Case D and A), the suction force seems to dominate the cross-draft leading to a more symmetric distribution of both flow and concentration fields. From this point of view, it can be concluded that the size and location of vortices are dependent on relative strength of the competing air currents (i.e., the cross-draft and the suction).

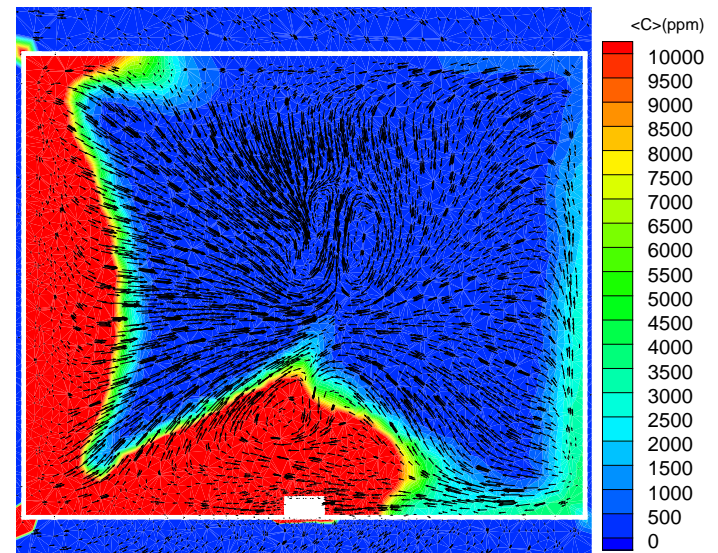

a) Low $V_{\text {Cross-draft }}$, Low $V_{\text {Face }}$

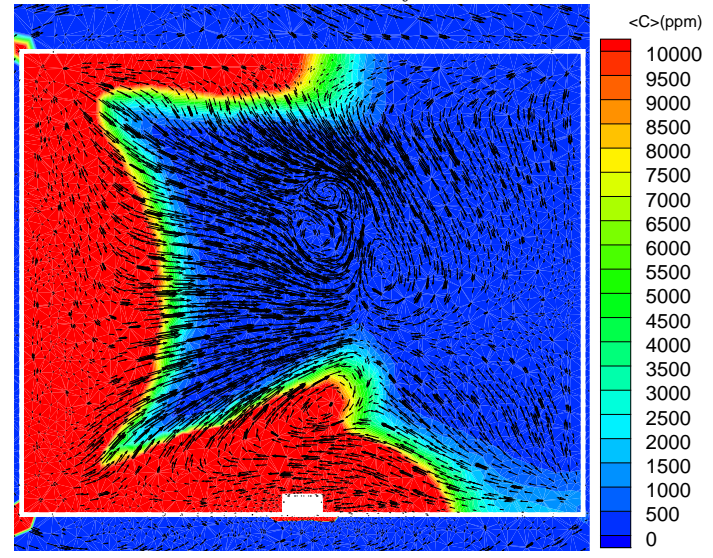

c) High $V_{\text {Cross-draft }}$, Low $V_{\text {Face }}$

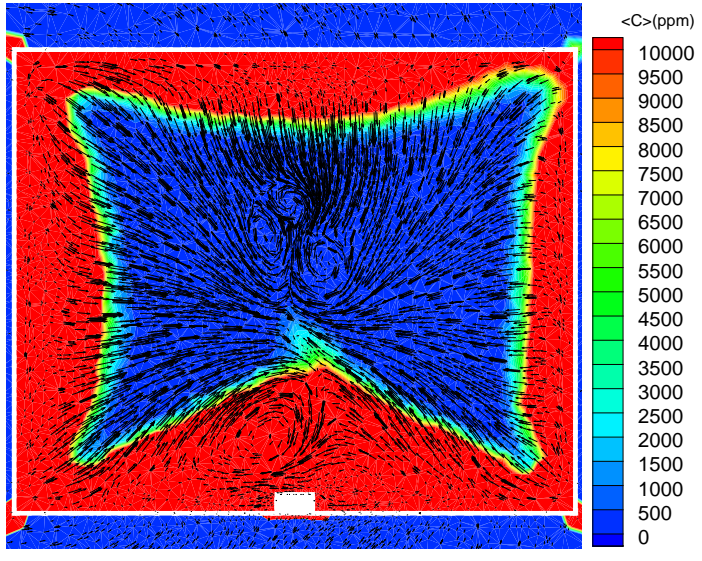

b) Low $V_{\text {Cross-draft }}$, High $V_{\text {Face }}$

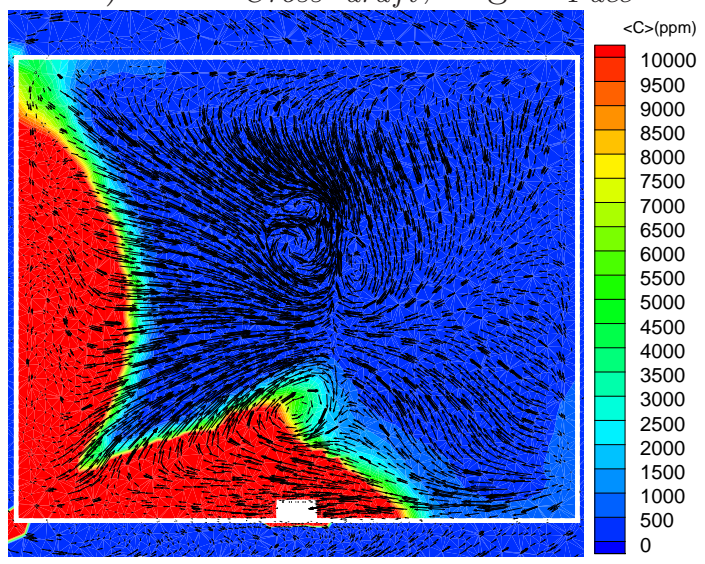

d) High $V_{\text {Cross-draft }}$, High $V_{\text {Face }}$

Figure 4.8: Flow and concentration fields at the hood face a) Case D, b) Case A, c) Case G, d) Case F 


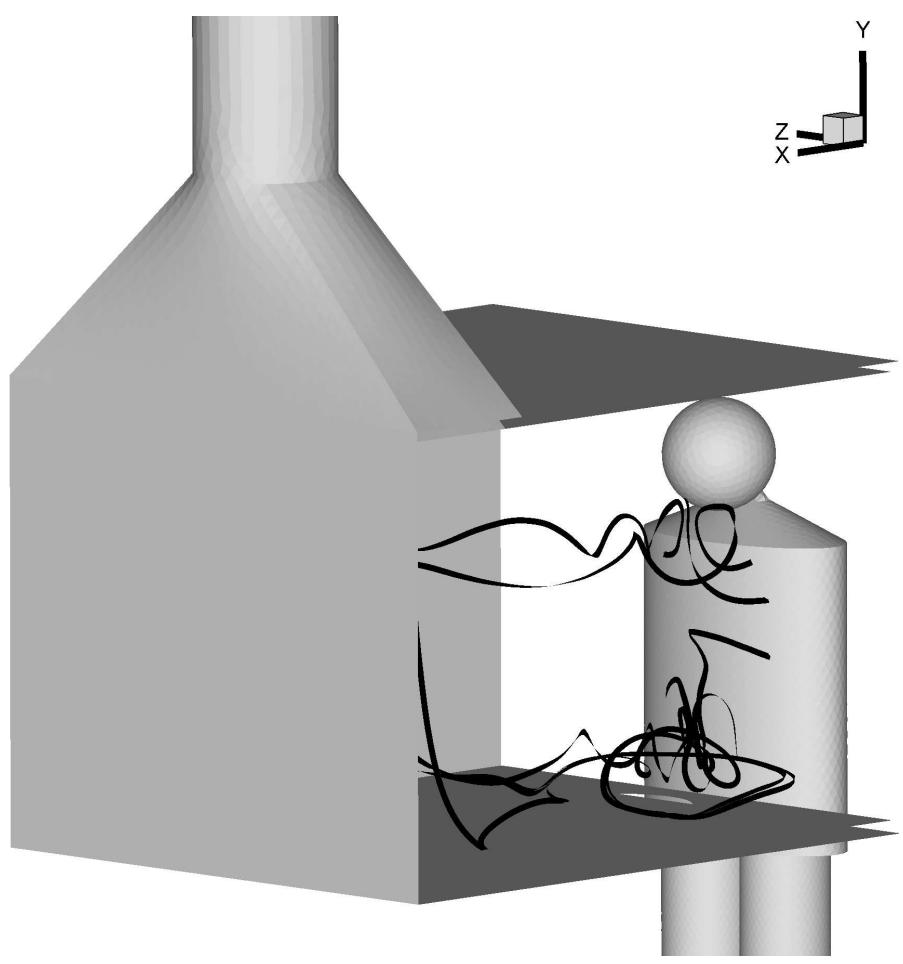

Figure 4.9: Three-dimensional stream-traces

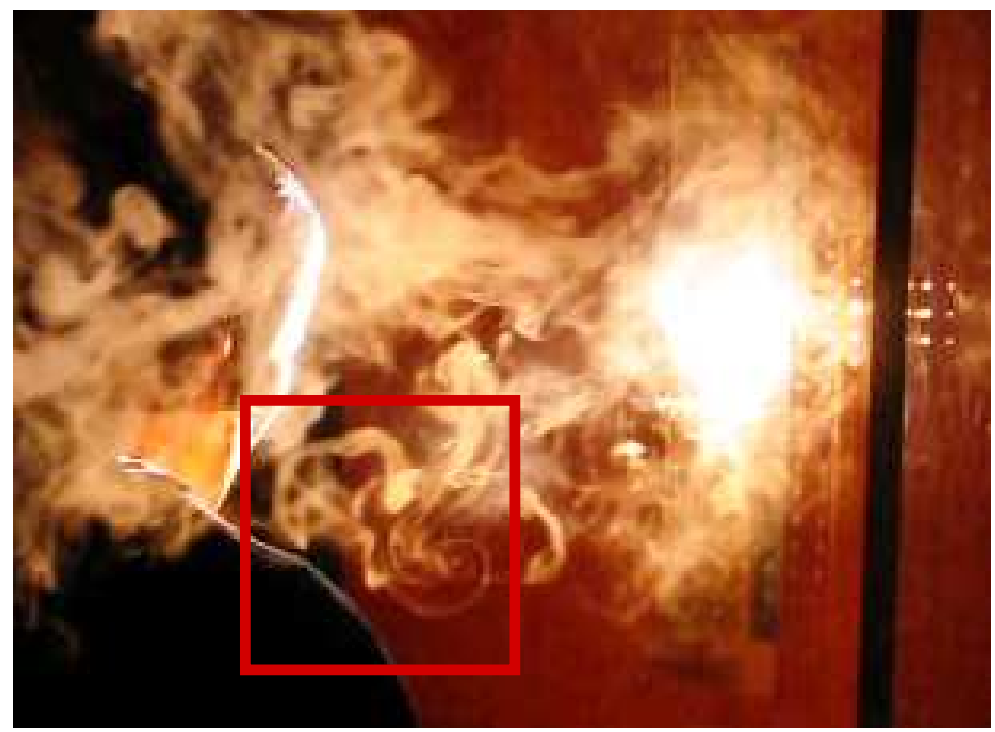

Figure 4.10: Snapshot showing the vortices in front of the worker (Case D) 
To further elucidate how the contaminant is transported within the hood, the long-time-averaged flow and concentration fields are depicted on another $x y$-plane further downstream of the hood ( $x y$-plane-2)(See Figure 4.11). From the figure, it is apparent that the vortex at the waist level becomes even larger further downstream and the contaminants released from the source are transported by this vortex towards the left wall, where they are trapped within the recirculation zones attached to the hood's walls. These recirculation zones arise from separation of the converging airflow at the front edges of the hood's side walls. Figure 4.12 shows the experimentally observed recirculation zone attached to the hood's left wall. As can be seen from Figure 4.8, the accumulated contaminant in these recirculation zone eventually moves up to the top wall of the hood. In this regard, one can conclude that the flow pattern inside the hood leads the contaminant to accumulate near the walls. One striking feature seen in Figure 4.8 is that compared to the generally high concentrations near the walls, the central region of the hood face remains relatively clean. The reason for this favorable situation is that the clean ambient air converging to the hood tends to enter the hood through the central region as can be easily seen from the vector fields shown in Figure 4.8. Once the air enters the hood through the central region, it is streamlined towards the back of the hood, minimizing the mixing of the near-wall contaminants across the hood face. This behavior of the flow was confirmed during flow visualization. The smoke trace in Figure 4.13 shows how the drawn clean air is streamlined towards the back of the hood without mixing, at least near the hood face.

Figure 4.14 displays the numerically predicted average flow and concentration fields on a horizontal plane (xz-plane) at the chest level. The predicted average flow field represented by the stream-traces appears to agree well with the experimental observations shown in Figures 4.12 and 4.13. Inspection of Figure 4.14 also indicates that when the suction force dominates the cross-draft (Figure $4.14 \mathrm{~b}$ ) or when they are comparable (Figure 4.14a), the flow inside the hood tends to be more symmetric with two recirculation zones attached to the 


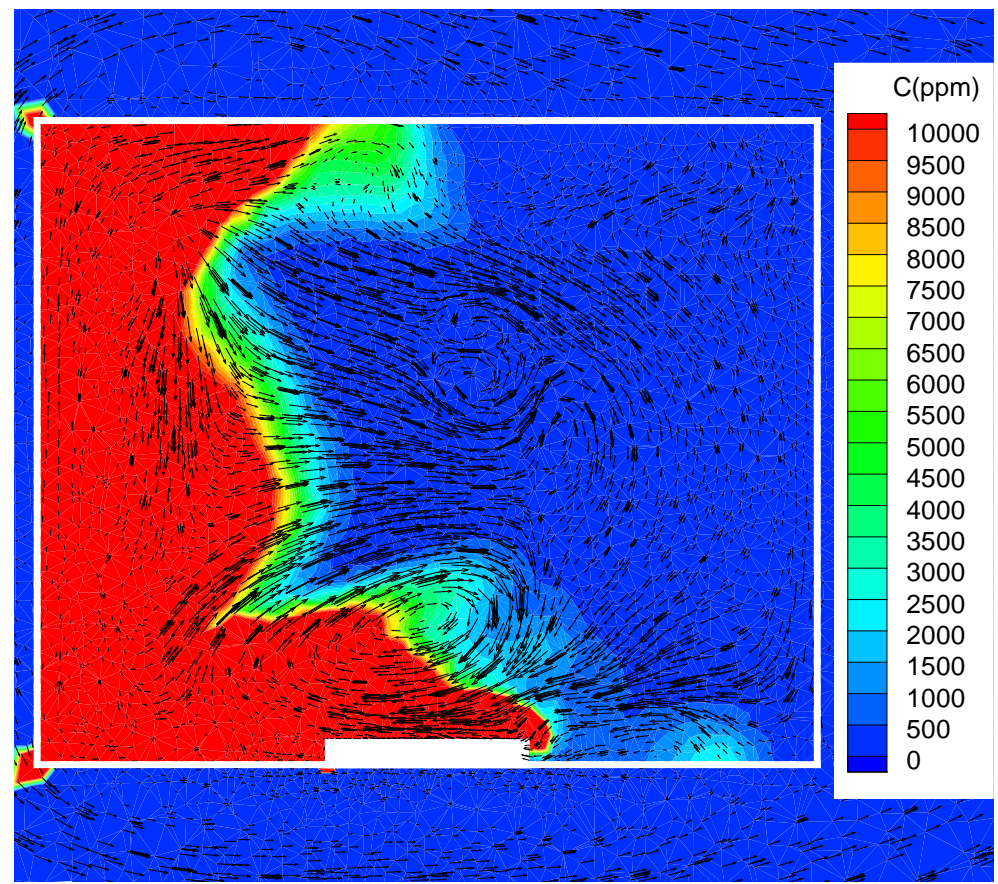

Figure 4.11: Flow and concentration fields for Case $\mathrm{G}$ at $z=1.7 \mathrm{~m}$

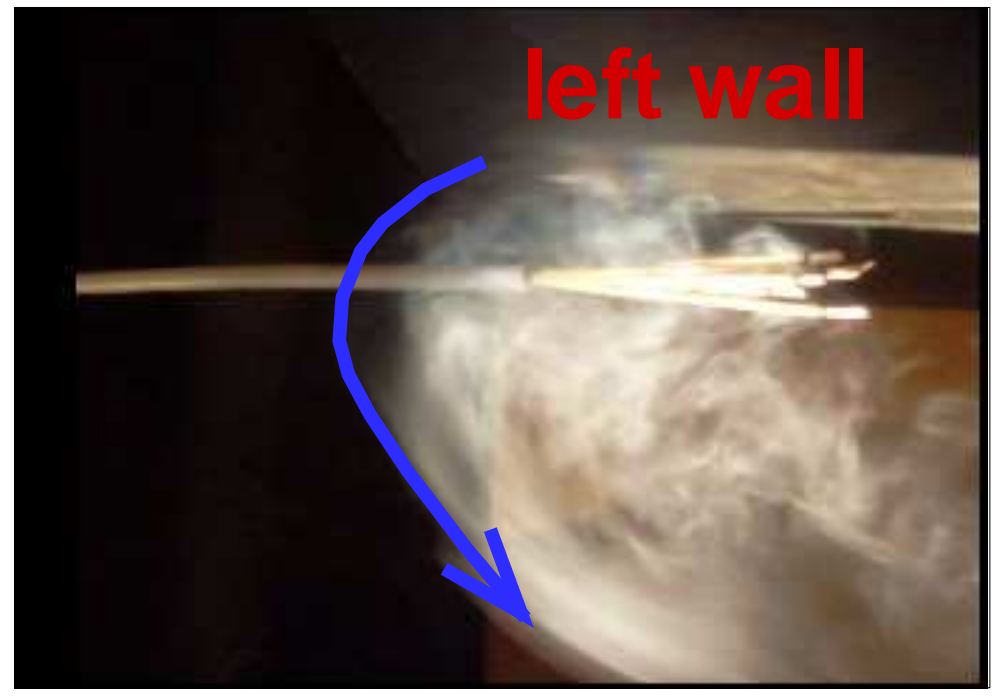

Figure 4.12: Snapshot showing the recirculation zone attached to the left wall of the hood (Case A, top view)

side walls. However, when the effect of cross-draft prevails over the suction force (Figure $4.14 \mathrm{c}$ and $4.14 \mathrm{~d}$ ), the size of the recirculation zone attached to the left wall increases while the recirculation zone attached to the right wall diminishes. The long-time-averaged concentration contours shown in the figure again shows how the concentrations are trapped within the recirculation zones. Interestingly, 


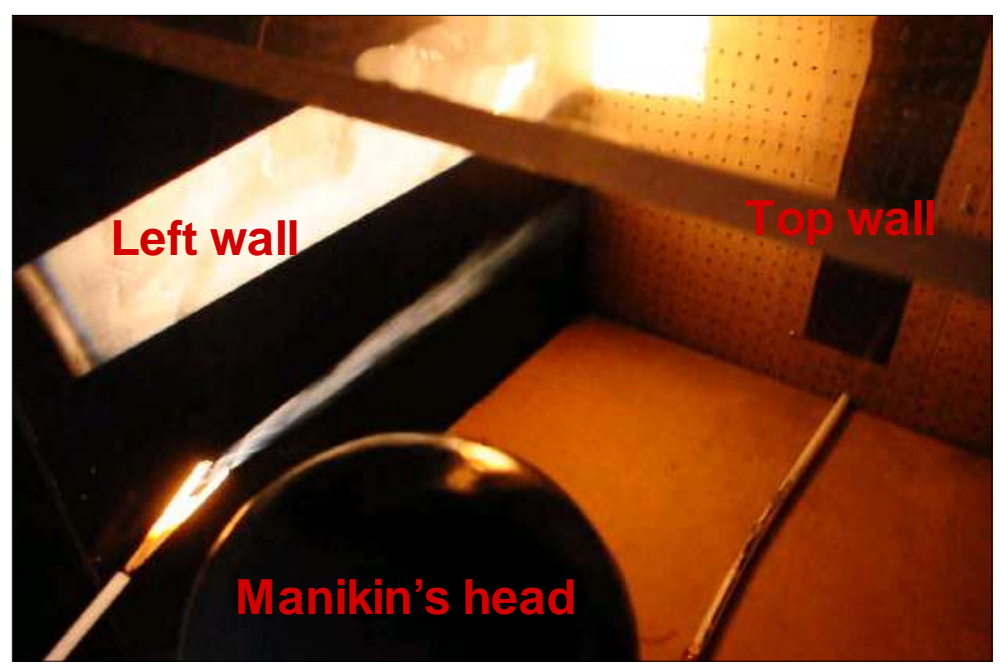

Figure 4.13: Trace of smoke streamlined towards the back of the hood (Case D)

Figure 4.14 reveals that there is no significant flow separation around the worker, and hence, no recirculation zone extending into the hood. It seems that the acceleration of the flow into the hood, to a large extent, hinders flow separation around the worker, and hence, also the formation of lateral vortices. From the figure it is seen that some very small lateral vortices in the immediate wake of the worker are formed. Indeed, the flow field observed through smoke visualization confirms the absence of the significant separation that would lead to the recirculation bubble around the manikin's torso. However, as shown in Figure 4.15a, an unsteady flow separation leading to a small recirculation zone confined in a small region was detected in the immediate downstream of the manikin's head. This phenomena could not be captured in the simulations as shown in Figure 4.15b.

Figure 4.16 illustrates the average flow patterns and concentration field on the hood's vertical symmetry plane (yz-plane-1). Again, there is no indication of the presence of lateral recirculation zone induced by the worker. The streamtraces point out that the vortices seen at the shoulder level (See Figure 4.8) are drawn directly toward the back of the hood under the effect of suction. Furthermore, an upward stream right in front of the worker is seen as well as the large vortical motion on the work surface. The flow visualization experiments confirm this predicted airflow patterns (see Figure 4.17). The upward flow of air 


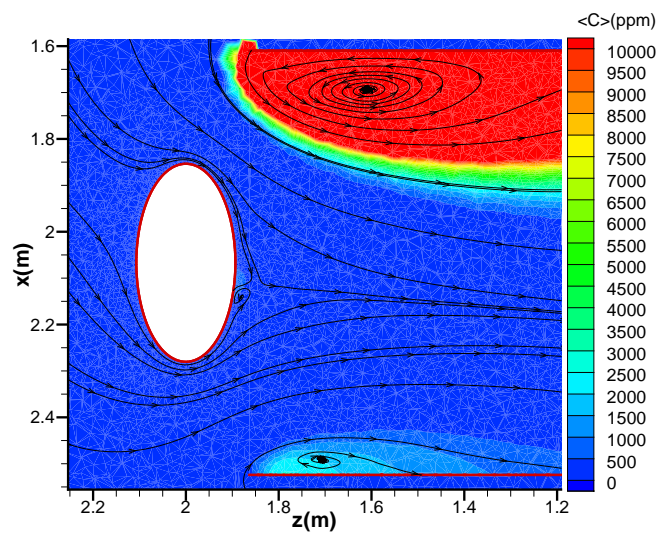

a) Low $V_{\text {Cross-draft }}$, Low $V_{\text {Face }}$

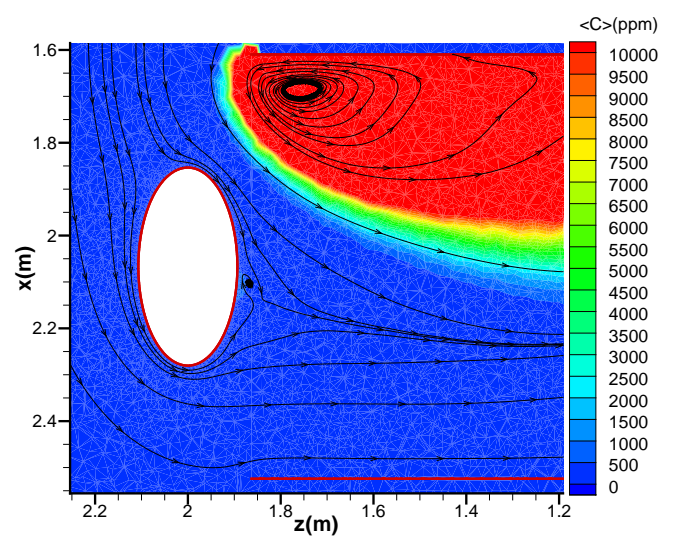

c) High $V_{\text {Cross-draft }}$, Low $V_{\text {Face }}$

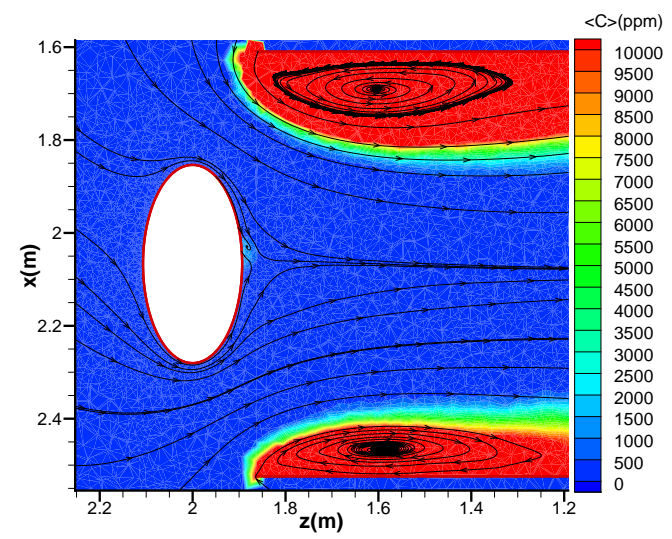

b) Low $V_{\text {Cross-draft }}$, High $V_{\text {Face }}$

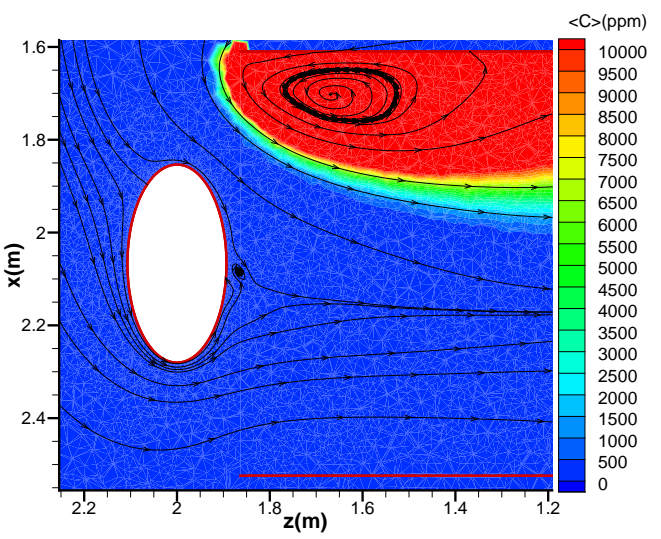

d) High $V_{\text {Cross-draft }}$, High $V_{\text {Face }}$

Figure 4.14: Airflow flow patterns and concentration fields on the $x z$-plane a) Case D, b) Case A, c) Case G, d) Case F

is expected to carry the contaminants to the worker's breathing zone. Although, it was mentioned that the worker's breathing zone is cleaner compared to nearwall regions, one should be aware that there could be still considerable level of contamination in the worker's breathing zone.

In a further attempt to elucidate this issue, the contours of long-timeaveraged concentration on the surface of the worker's body are plotted in Figure 4.18 with an adjusted color scale. From the figure it is seen that the worker's face is exposed to contaminants. Apparently, at higher cross-draft (Cases $\mathrm{G}$ and $\mathrm{F}$ ) the exposure level is less. It is also worth noting that the concentration level changes considerably over the area of manikin's face. This 


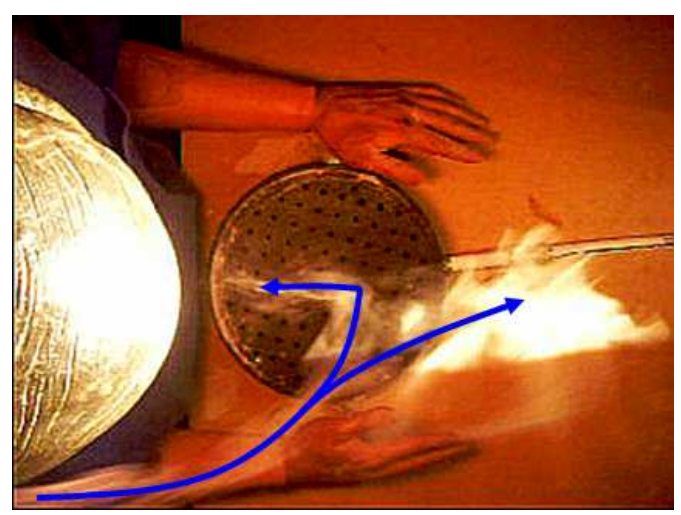

a)

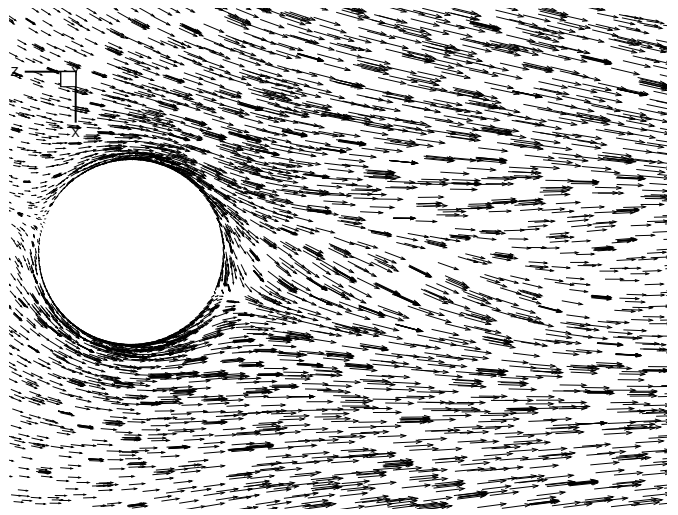

b)

Figure 4.15: Comparison of experimentally observed and numerically predicted flow fields around the worker's head (Case D) a) Experimental b) Numerical

indicates to several critical issues; i) the concentration level at a given monitoring location can vary depending on the body shape, ii) the monitoring location is important, iii) in practice, depending on the movement/position of worker's head, the exposure level can change significantly.

It is well known that presence of vortices at the hood face are indicative of the presence of turbulence. Figure 4.19 shows the distribution of turbulent kinetic energy to quantify the extent of turbulence present at the hood face. As expected, the turbulent kinetic energy is high in regions where vortical motions are present; in front of the worker and in the vicinity of the walls. Furthermore, the turbulent kinetic energy is higher in high $V_{\text {Face }}$ cases compared to low $V_{\text {Face }}$ cases. To this end, it can be concluded that high $V_{\text {Face }}$ may not be desirable due to more mixing. It is very probable that the turbulent diffusion plays an important role in the transport of contaminants to the worker's breathing zone. Figure 4.20 illustrates the experimentally observed turbulence in the worker's breathing zone. 


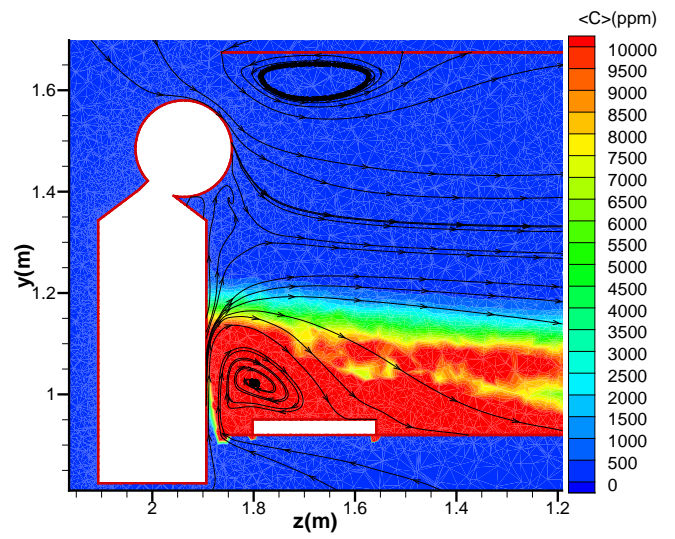

a) Low $V_{\text {Cross-draft }}$, Low $V_{\text {Face }}$

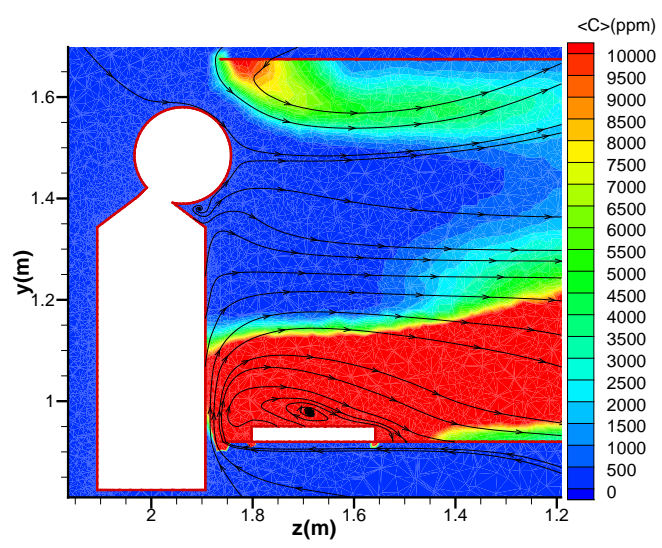

c) High $V_{\text {Cross-draft }}$, Low $V_{\text {Face }}$

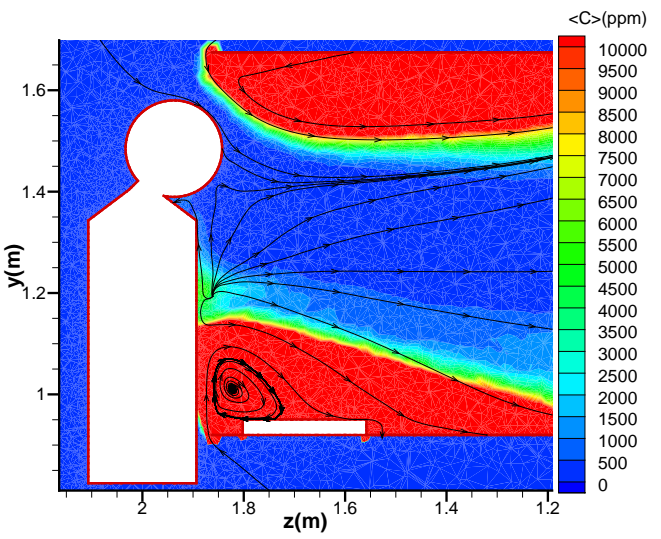

b) Low $V_{\text {Cross-draft }}$, High $V_{\text {Face }}$

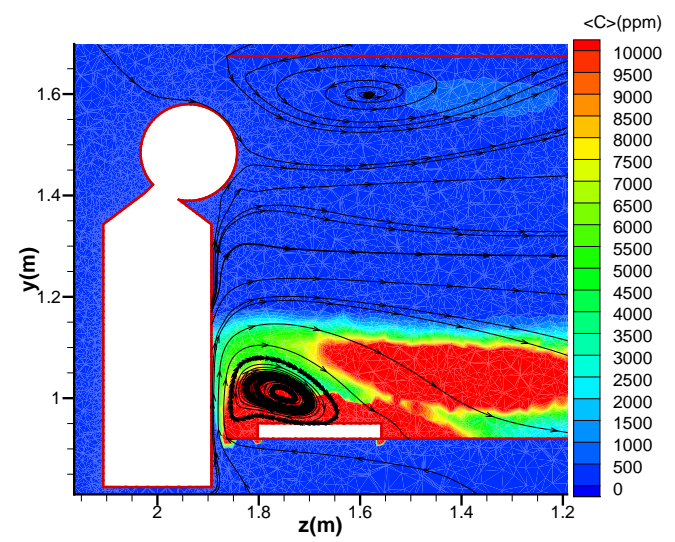

d) High $V_{\text {Cross-draft }}$, High $V_{\text {Face }}$

Figure 4.16: Airflow flow patterns and concentration fields at the hood face a) Case D, b) Case A, c) Case G, d) Case F

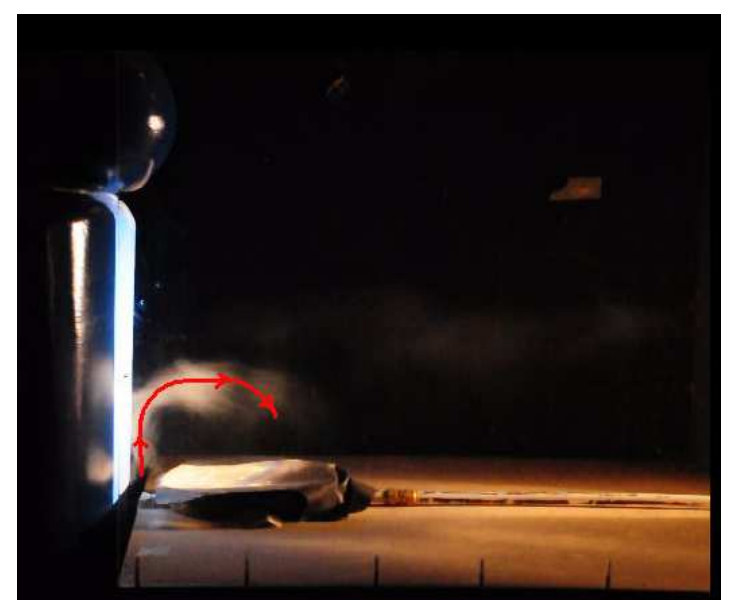

Figure 4.17: Vortical motion above the work surface (Case A) 


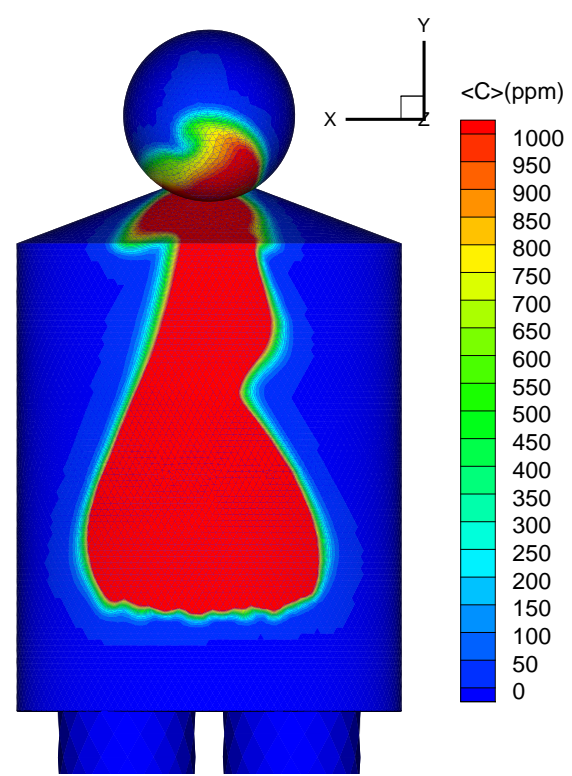

a) Low $V_{\text {Cross-draft }}$, Low $V_{\text {Face }}$

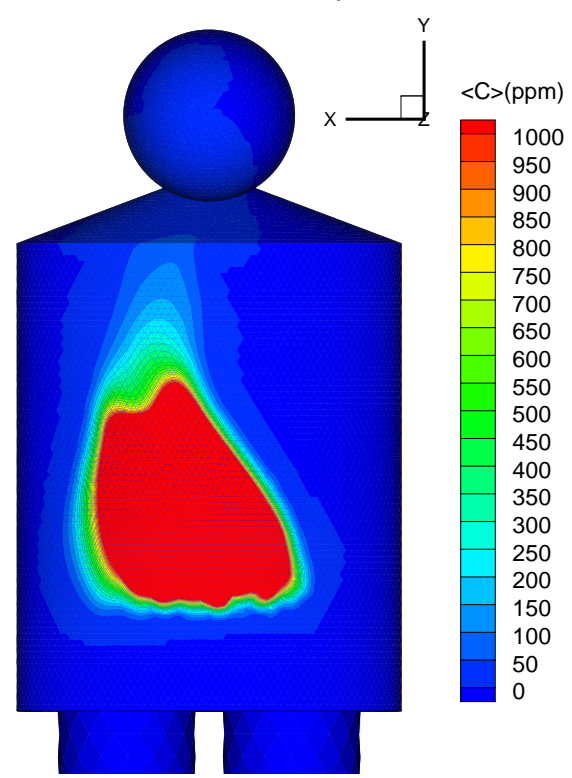

c) High $V_{\text {Cross-draft }}$, Low $V_{\text {Face }}$

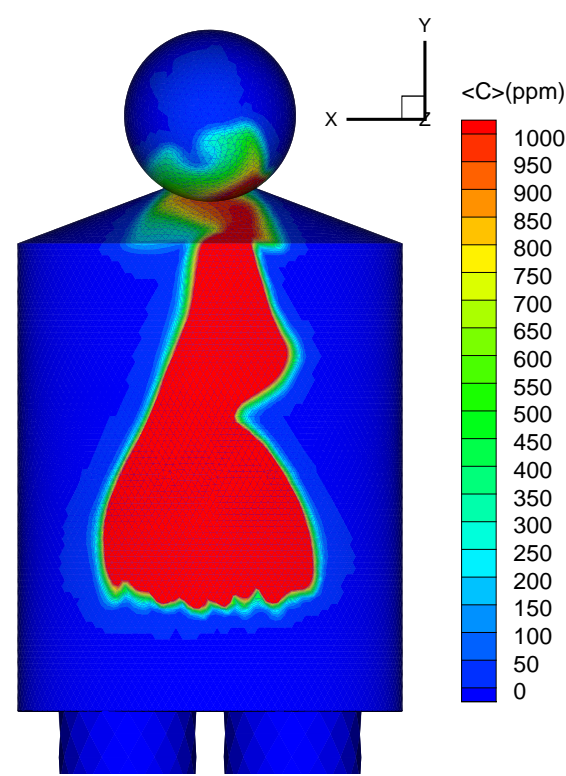

b) Low $V_{\text {Cross-draft }}$, High $V_{\text {Face }}$

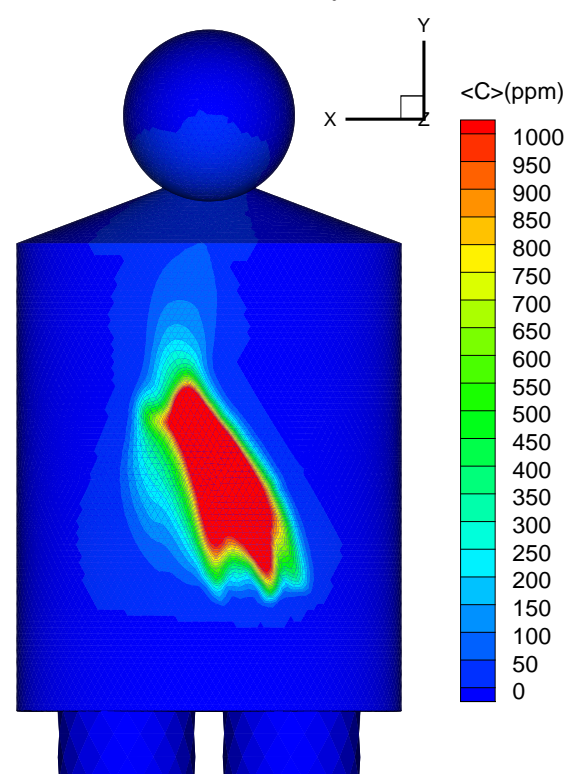

d) High $V_{\text {Cross-draft }}$, High $V_{\text {Face }}$

Figure 4.18: Concentration distributions on the surface of the worker (manikin) a) Case D, b) Case A, c) Case G, d) Case F 


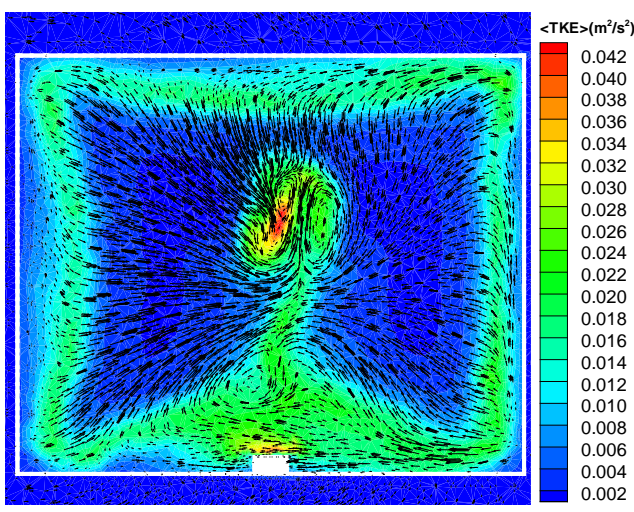

a) Low $V_{\text {Cross-draft }}$, Low $V_{\text {Face }}$

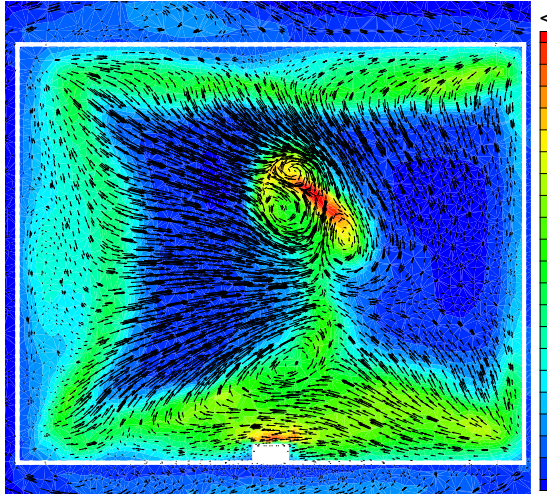

\begin{tabular}{|c}
$<$ TKE $>\left(\mathbf{m}^{2} / \mathbf{s}^{2}\right)$ \\
0.042 \\
0.040 \\
0.038 \\
0.038 \\
0.036 \\
0.034 \\
0.032 \\
\hline 0.030 \\
\hline 0.028 \\
0.026 \\
0.024 \\
0.022 \\
0.020 \\
0.018 \\
0.016 \\
0.014 \\
0.012 \\
0.010 \\
0.008 \\
0.006 \\
0.004 \\
0.002
\end{tabular}

c) High $V_{\text {Cross-draft }}$, Low $V_{\text {Face }}$

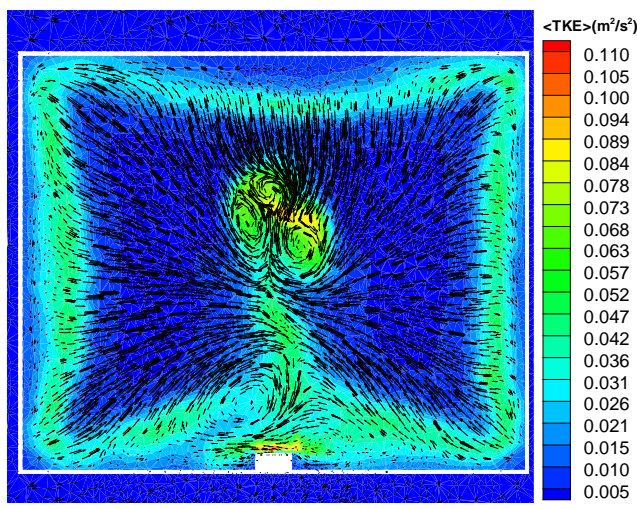

b) Low $V_{\text {Cross-draft }}$, High $V_{\text {Face }}$

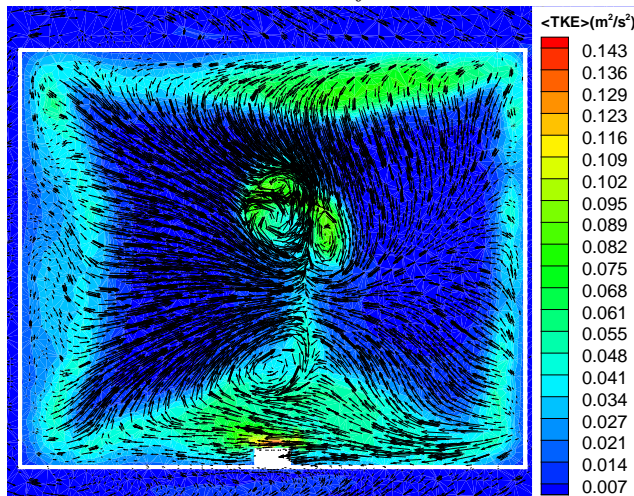

d) High $V_{\text {Cross-draft }}$, High $V_{\text {Face }}$

Figure 4.19: Flow and turbulent kinetic energy fields at the hood face a) Case D, b) Case A, c) Case G, d) Case F

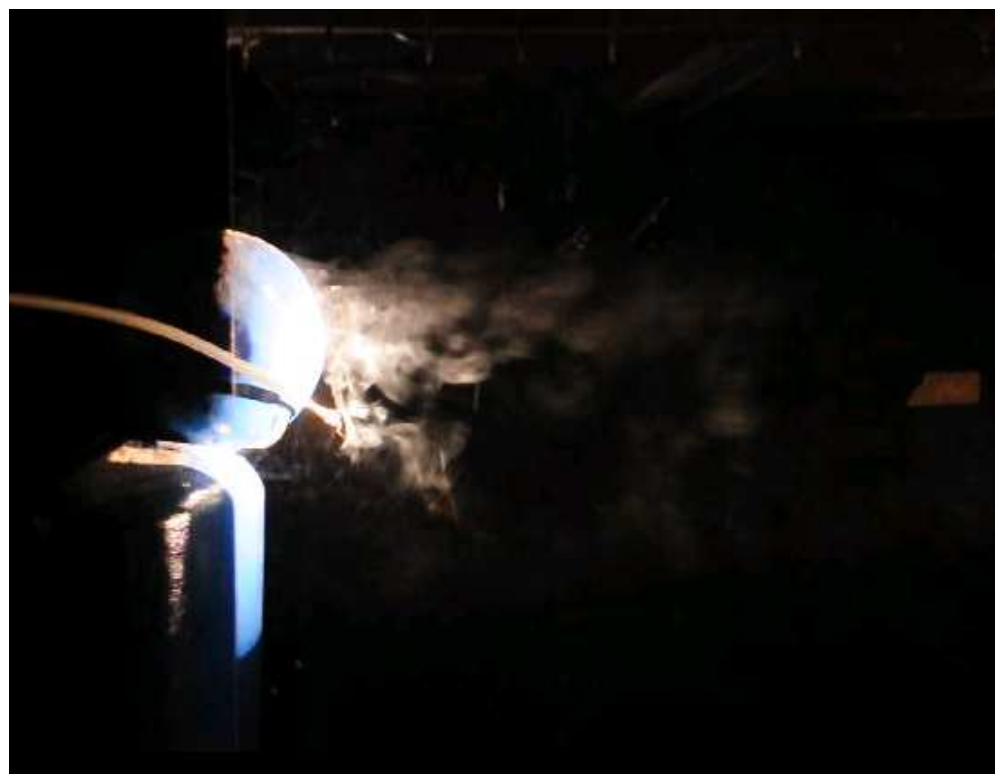

Figure 4.20: Mixing in the breathing zone of the manikin (Case D) 


\subsubsection{Flow unsteadiness}

Before discussing the instantaneous results, it is noteworthy to mention that for a given condition the exposure measurements taken in the wind tunnel exhibit high variability despite a quite long sampling period (15min) [52]. Unless there are variations in the experimental conditions with a period larger than the sampling period, the repeated measurements are expected to fall into a close band. Based on the size of the wind tunnel and the flow rates used, $15 \mathrm{~min}$ of sampling period seems long enough to damp out-of-hand variations in the flows. In this respect, obtaining highly variable results even with $15 \mathrm{~min}$ of sampling period poses a challenging problem that needs to be addressed.

Figure 4.21 in which the stream-traces and vorticity contours from two different time steps are shown, reveals that the small vortices seen at the hood face are dynamic. Also the tumbling eddies depicted in Figure 4.22 indicate that large dynamic eddies characterize the flow inside the hood. In short, even under ideal and isolated simulation conditions dynamic flow structures are established. Considering that all the boundary conditions are steady, these dynamic flow structures point to self-induced unsteadiness.

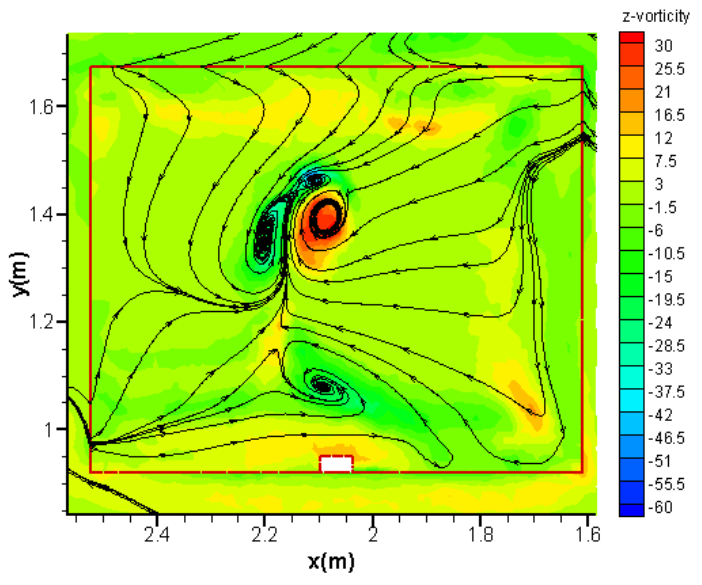

a)

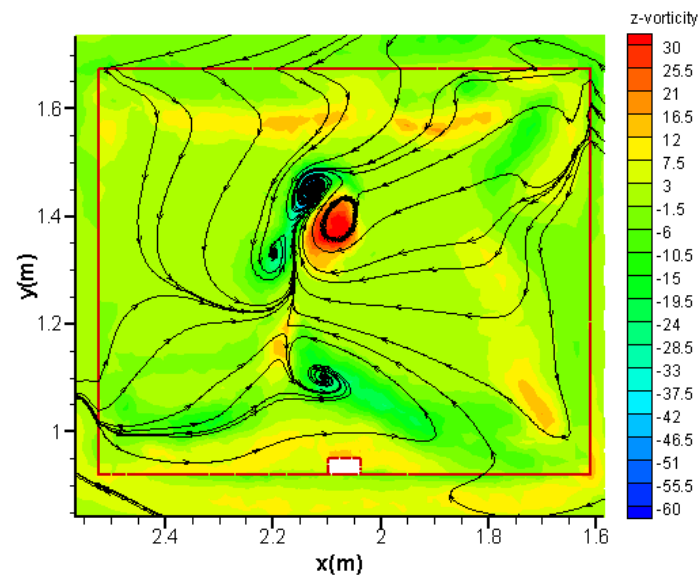

b)

Figure 4.21: Vorticity contours and stream-traces at the hood face for Case G a) $t=22 \mathrm{~s}$, b) $t=23 \mathrm{~s}$ 


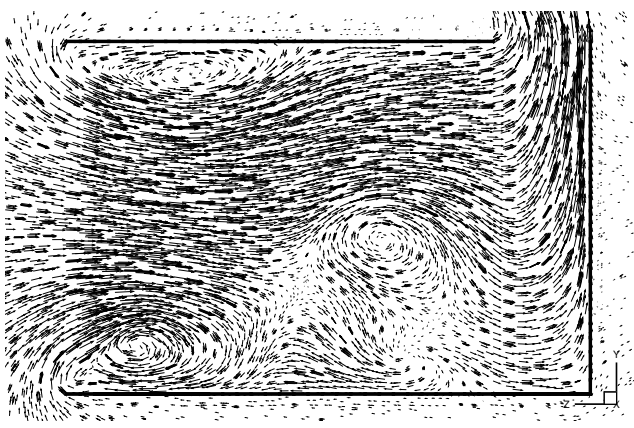

a)

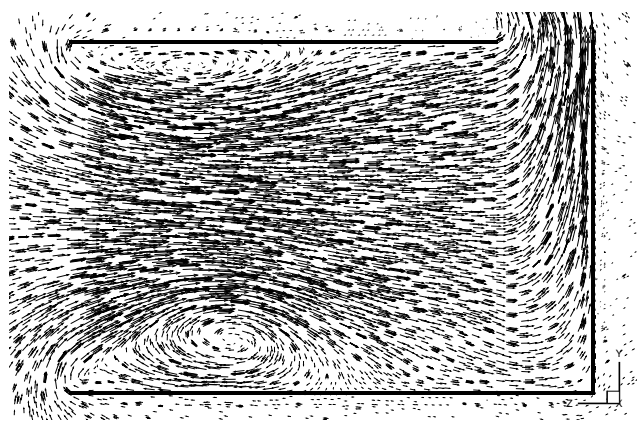

b)

Figure 4.22: Eddies tumbling on a vertical plane on the right of manikin (yz-plane-2, Case G) a) $t=65 \mathrm{~s}$, b) $t=67 \mathrm{~s}$

The time history of the concentration and $z$-velocity power spectrums at Point 1 and Point 2 are given in Figures 4.23 to 4.30 for Cases A, D, F and G. As can be seen from the figures, there are variabilities in the instantaneous concentration that sometimes reach up to $50 \%$ or more. The factors that can change from experiment to experiment such as air temperature, boundary conditions, etc. can have an augmenting effect on the observed variability. In addition, the factors like body heat, breathing and their non-linear interaction may increase the variability even more. The best way to understand what indeed causes this situation would be to perform the experiments under fully controlled conditions. However, since the conditions in working environments generally are not fully controlled, the benefit of performing such an experiment might be questionable.

The unsteady character of the predicted flow can be deduced from the power spectrums of the velocity and concentration fluctuations presented in Figures 4.23 to 4.30 . Since the points at which data are monitored are in front of the spherical head, uniform flow around a sphere can be used as an approximate reference case in assessing the predicted behavior of oscillations. It is known that for uniform flow past a sphere two dominant frequencies coexist. The Strouhal number for low frequency is around 0.2 and is associated with larger scale instability of the wake. The high frequency oscillations, on the other hand, are caused by the small scale instability from separation of the shear 
layer [55]. Although the present case is different than the uniform flow past a sphere, the power spectrums also indicate existence of, in general, two significant frequencies. The low frequencies for Cases D, E, F are in the range of about $0.3-0.8 \mathrm{~Hz}$. This corresponds to a Strouhal number range of 0.11-0.23, which is comparable to the Strouhal number associated with the low frequency (0.2) in free-stream. Furthermore, the unsteadiness observed in the smoke tests for Case D indicates that the flow separated from the simple manikin's spherical head oscillates at a frequency of about $0.2 \mathrm{~Hz}$. This frequency corresponds to a Strouhal number of 0.26 , further confirming the accuracy of the simulations. In the simulations, the Strouhal numbers for high frequencies, on the other hand, take values from about 0.3 to 1.7. For Case A, however, the predicted frequencies are relatively higher, indicative of a more turbulent flow. This high frequency might be attributed to the strong suction effects created by the hood. It is worth noting that in two-dimensional simulations (Chapter 3) the effect of suction was found to cause very high frequencies. Although in those simulations the flow was coming from the back of the ellipse representing the worker, still the three-dimensional, unsteady, vortical flow structures seen in three-dimensional simulations sow further doubts on lost physics in the two-dimensional test case. Even so, two-dimensional test case may still serve well for sensitivity analysis of the RANS turbulence models for such flows. 

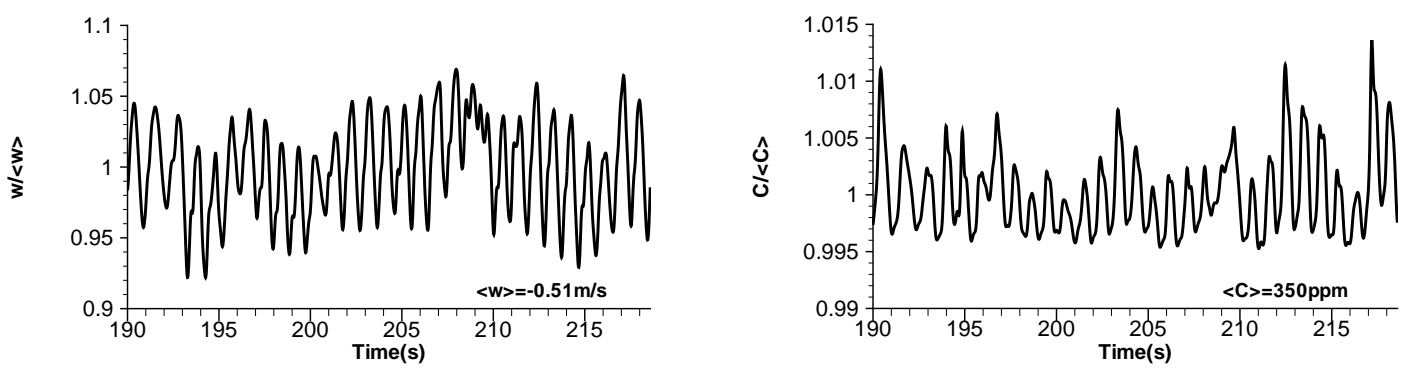

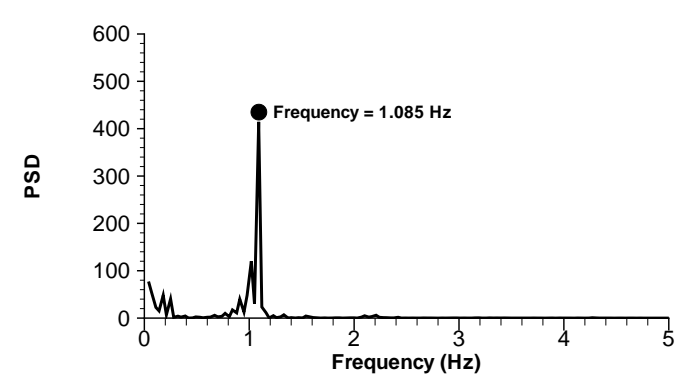

a)

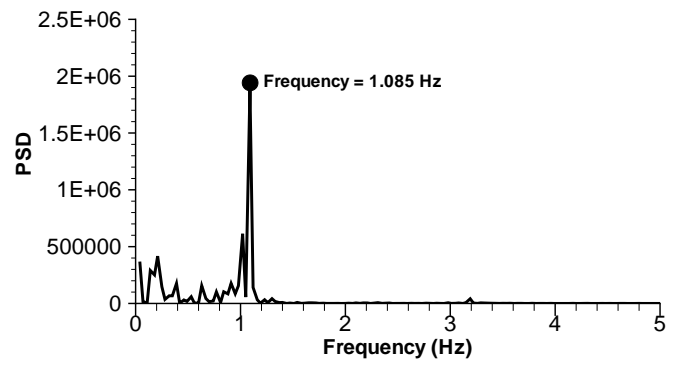

b)

Figure 4.23: Time history of $z$-velocity and concentration along with their PSD spectrum at Point 1 for Case D a) $z$-velocity b) concentration
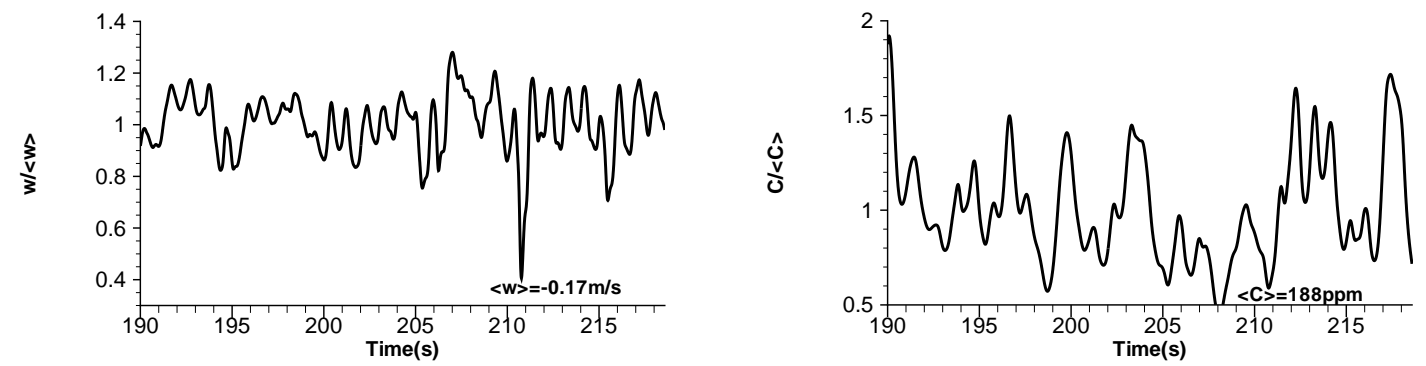

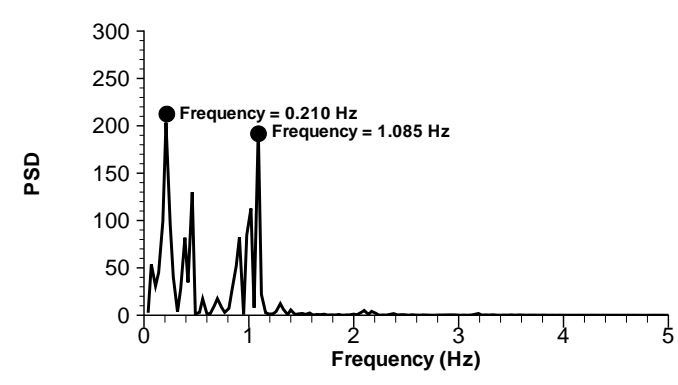

a)

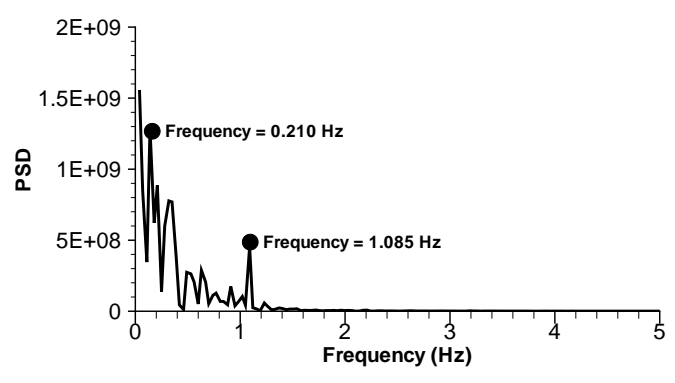

b)

Figure 4.24: Time history of $z$-velocity and concentration along with their PSD spectrum at Point 2 for Case D a) $z$-velocity b) concentration 

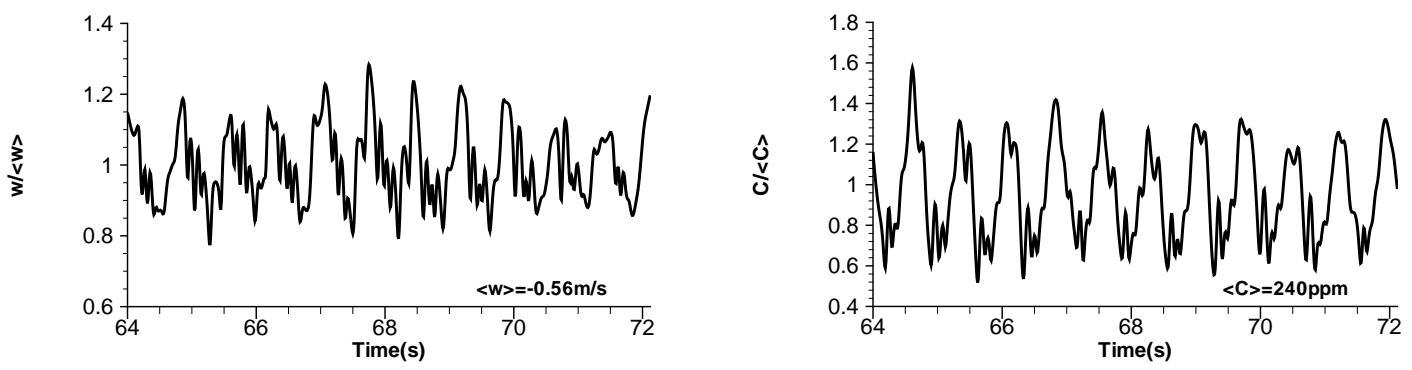

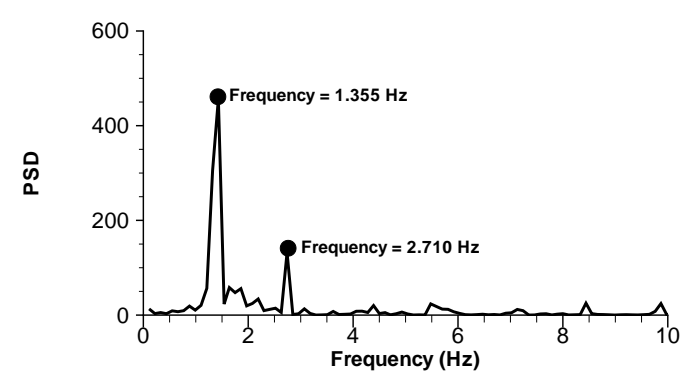

a)

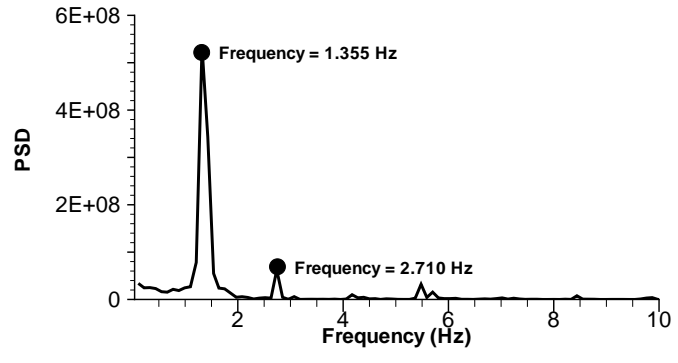

b)

Figure 4.25: Time history of $z$-velocity and concentration along with their PSD spectrum at Point 1 for Case A a) $z$-velocity b) concentration
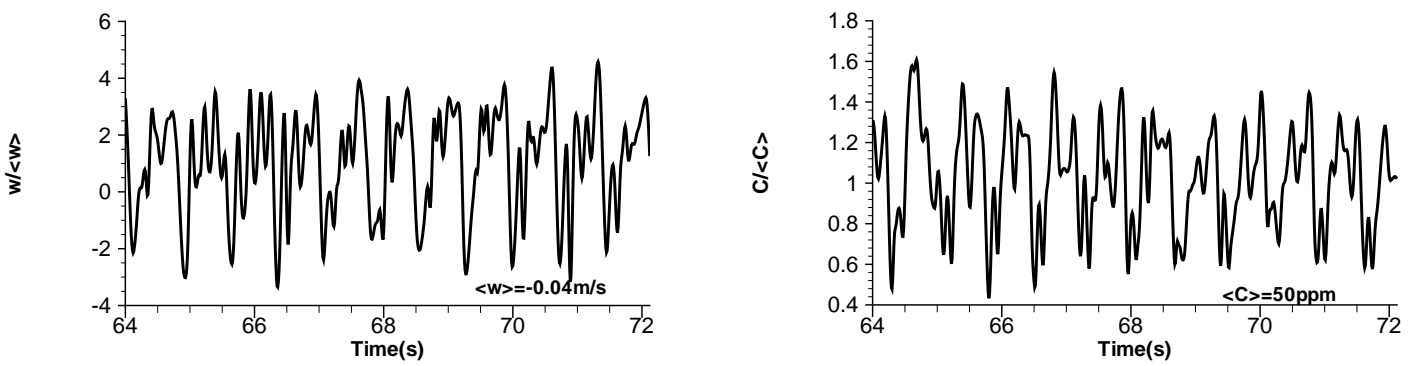

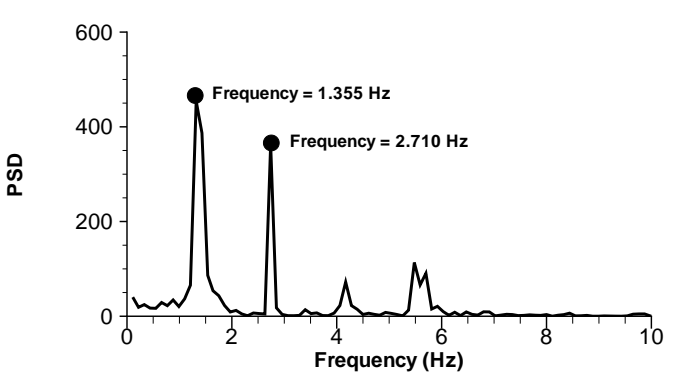

a)

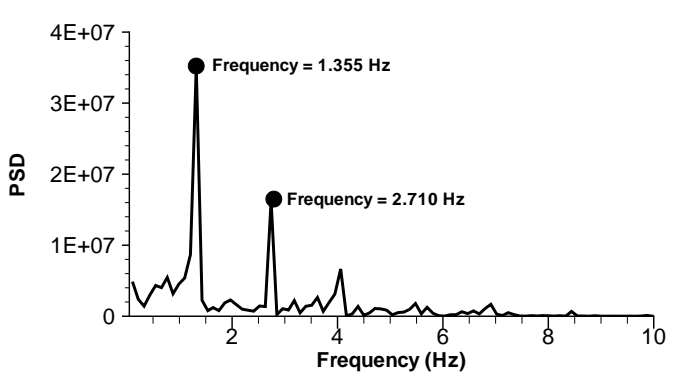

b)

Figure 4.26: Time history of $z$-velocity and concentration along with their PSD spectrum at Point 2 for Case A a) $z$-velocity b) concentration 

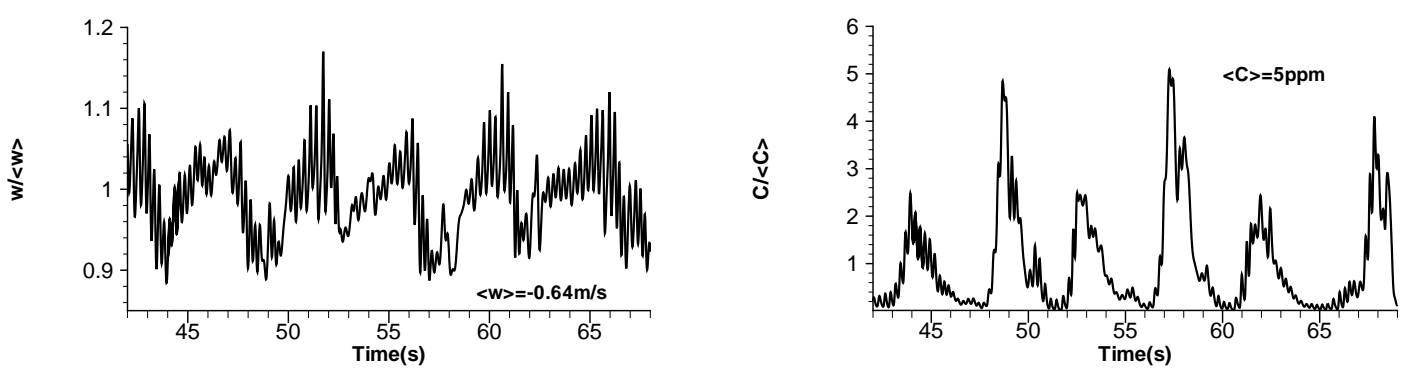

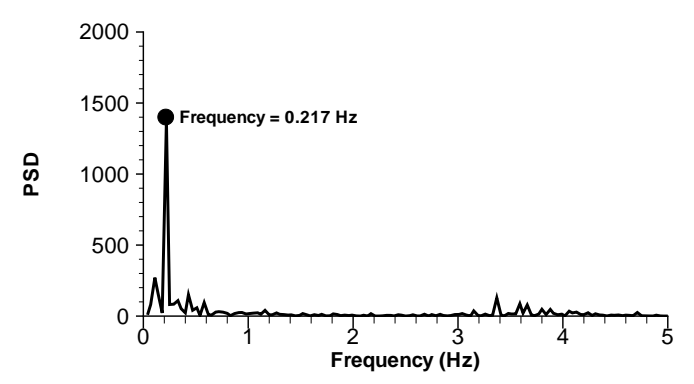

a)

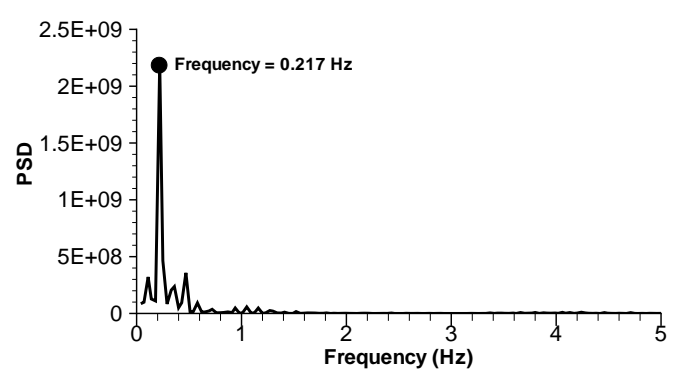

b)

Figure 4.27: Time history of $z$-velocity and concentration along with their PSD spectrum at Point 1 for Case G a) $z$-velocity b) concentration
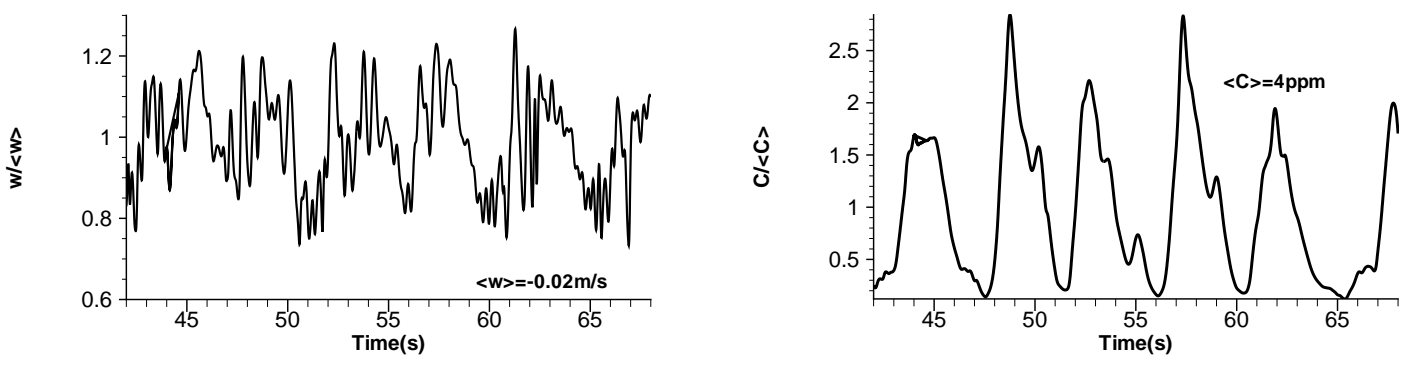

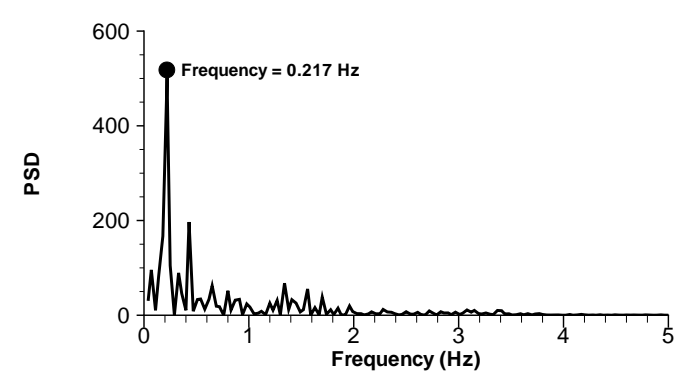

a)

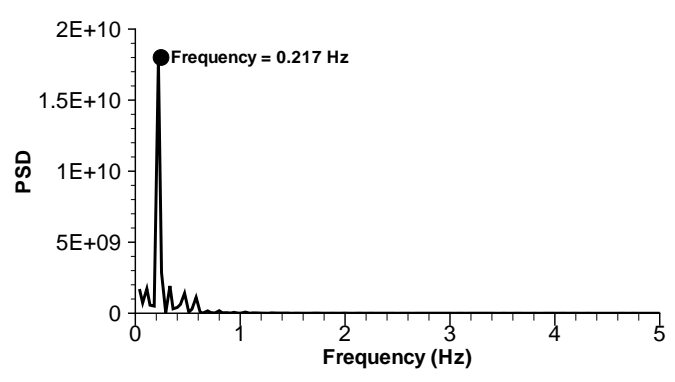

b)

Figure 4.28: Time history of $z$-velocity and concentration along with their PSD spectrum at Point 2 for Case G a) $z$-velocity b) concentration 

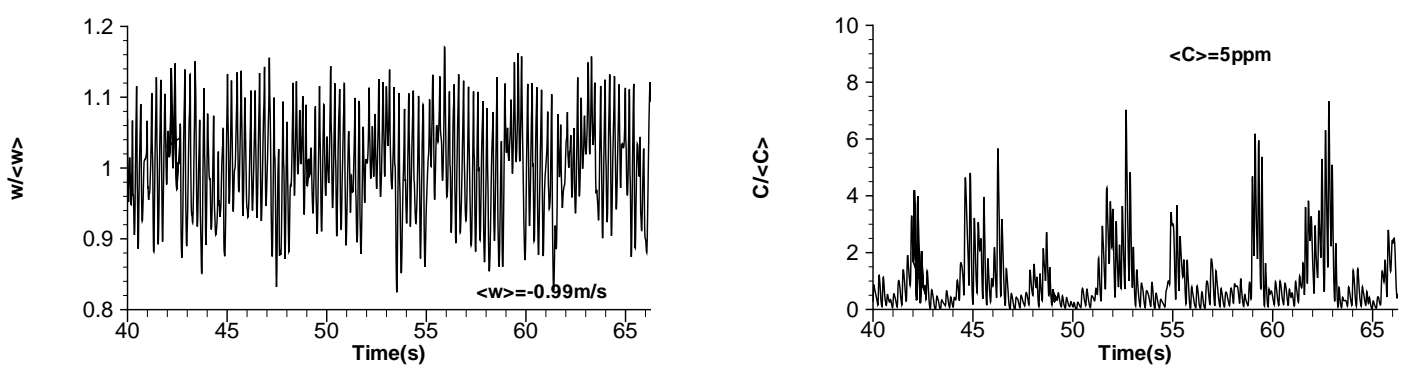

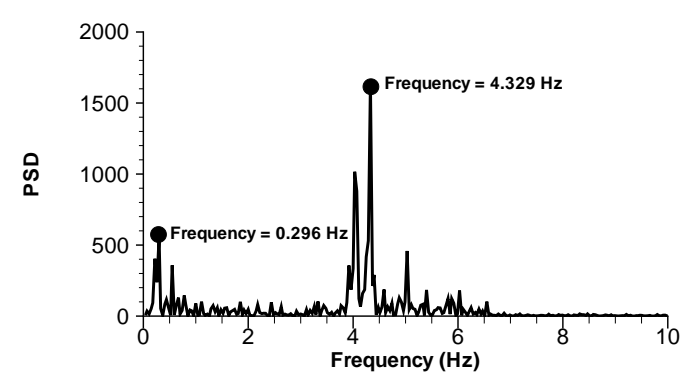

a)

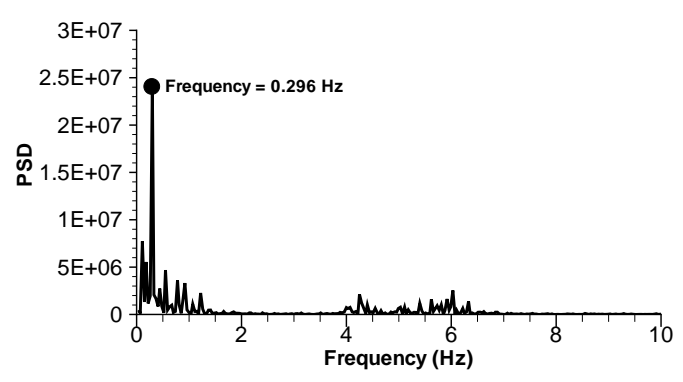

b)

Figure 4.29: Time history of $z$-velocity and concentration along with their PSD spectrum at Point 1 for Case F a) $z$-velocity b) concentration
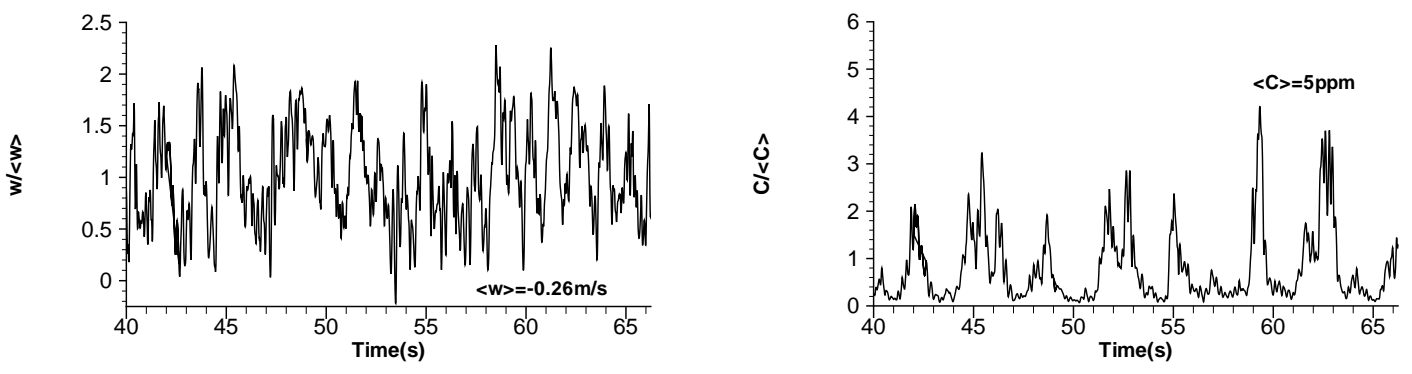

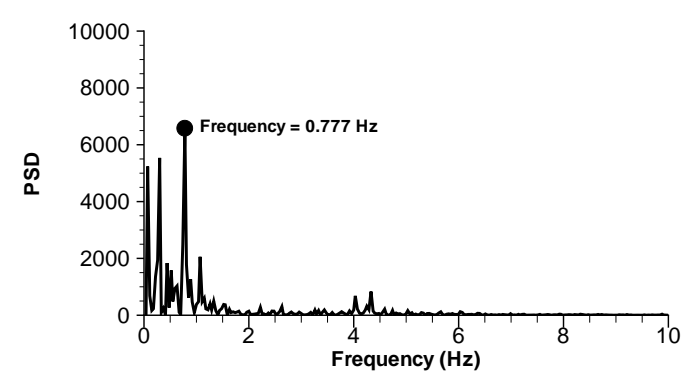

a)

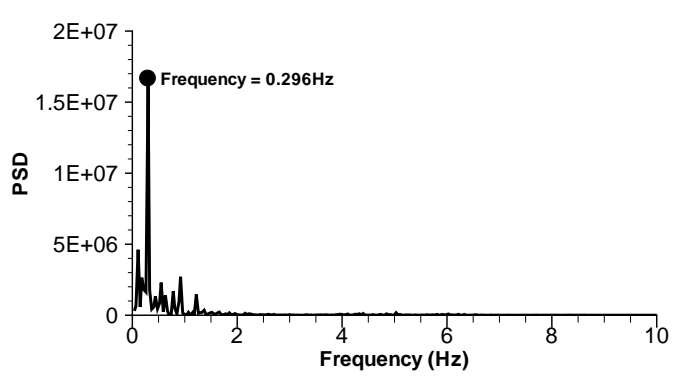

b)

Figure 4.30: Time history of $z$-velocity and concentration along with their PSD spectrum at Point 2 for Case F a) $z$-velocity b) concentration 
In an attempt to compare the flow and concentration level on both sides of the worker's line of symmetry which is straight down the middle, the time histories of predicted velocity and concentration level for Case D are plotted in Figure 4.31. Comparisons of Figure 4.31 with Figure 4.23 and 4.24 clearly show that the flow and concentration at Point 3 are very closely correlated with those at Point 1. Also, the time averaged concentration level at Point 3 is slightly higher compared to that at Point 1, due to its relatively shorter distance from the contaminant source. On the other hand, the time variations of flow and concentration level at Point 3 and Point 2 are not as much correlated as Point 3 and Point 1. This is expected because on the downstream side of the worker's line of symmetry, a pressure drop occurs and this leads to a change in the flow pattern, and hence, in the concentration.

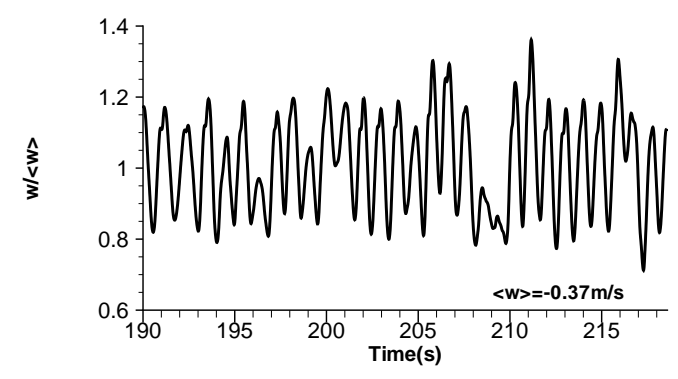

a)

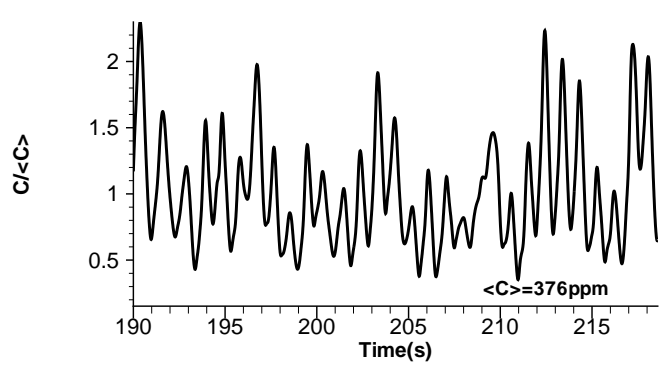

b)

Figure 4.31: Time history of $z$-velocity and concentration at Point 3 for Case D a) $z$-velocity b) concentration

\subsubsection{Effect of Direction of Draft}

In order to investigate the effect of draft direction on the wake flow, a simulation was performed for a flow configuration where the draft comes from the worker's back. The simulation was performed for only Case D and only the flow field was solved. The predicted time-averaged flow field is illustrated in Figure 4.32. Notably, for this flow configuration, lateral vortices induced by the presence of the worker are predicted. Remember that, on the previous flow configuration very small vortices were scarcely found in both simulations and experiments. It seems that the cross-draft has an inhibiting effect on the 
formation of vortices for the previous configuration. Here, however, the draft coming from the worker's back helps formation of the vortices.

In principle, these vortices are indicator of presence of low pressure region in front of the worker. Therefore, they can draw contaminants from elsewhere in the hood toward the worker. Over time, this may lead to accumulation of contaminant, and hence, higher levels of exposure. From this point of view, the present flow configuration seems to be a more severe case compared to the previous one.

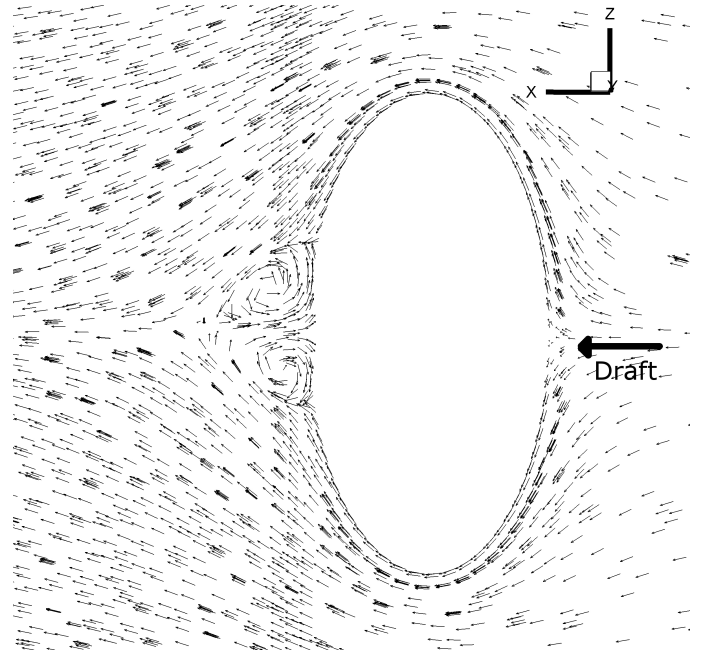

a)

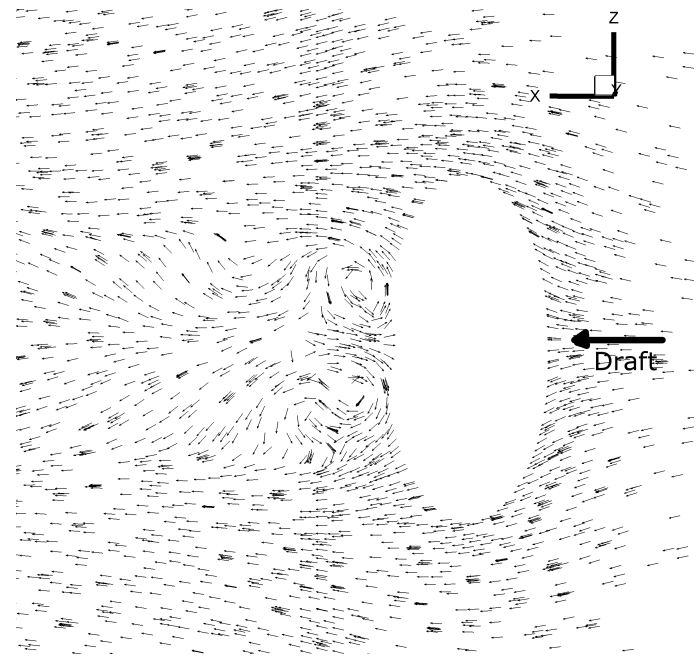

b)

Figure 4.32: Average flow field in the wake of the manikin for Case $\mathrm{G}$ a) waist level b) shoulder level

\subsubsection{Non-isothermal Flow}

Non-isothermal computations were carried out to investigate the thermal impact of worker's body on the airflow dynamics and exposure levels. Based on the average temperatures measured in the experiments, the temperatures of the manikin's head and neck were set to $300 \mathrm{~K}$, whereas the shoulder and torso were kept at 303K. The temperature for the manikin's legs and all other walls were set equal to the air temperature (297K). Thus the heat was released only from the upper body of the manikin to match the experimental conditions. 
Table 4.2: Calculated dimensionless quantities for various $V_{\text {Cross-draft }}$ to $V_{\text {Face }}$ ratios

\begin{tabular}{cccc}
\hline Case & $V_{\text {Cross-draft }} / V_{\text {Face }}$ & $R e$ & $R i$ \\
\hline $\mathrm{A}$ & 0.073 & 5,348 & 14.5 \\
$\mathrm{~B}$ & 0.086 & 5,051 & 16.25 \\
$\mathrm{C}$ & 0.106 & 4,754 & 18.34 \\
$\mathrm{D}$ & 0.133 & 4,456 & 20.87 \\
$\mathrm{E}$ & 0.186 & 10,102 & 4.06 \\
$\mathrm{~F}$ & 0.300 & 13,667 & 2.22 \\
$\mathrm{G}$ & 0.553 & 11,885 & 2.94 \\
\hline Grashof number & Gr: $4.1 \times 10^{8}$ & &
\end{tabular}

Grashof number, $G r: 4.1 \times 10^{8}$

Since there are cross-draft and suction by the hood in the present problem, both natural convection and forced convection coexist. It is known that increased air flowrates decreases the relative importance of buoyancy effect associated with natural convection. Of particular interest are the conditions at which buoyancy effects are effective. As mentioned earlier, Richardson number $\left(R i=G r / R e^{2}\right)$ is the dimensionless quantity used to determine the relative strength of natural convection with respect to forced convection. Generally, if $R i>>1$ natural convection effects overwhelm forced convection. Based on the lowest and highest approaching velocities and the shoulder diameter of the manikin, the Reynolds number $(R e)$ is found to be in the range of 4,500-13,700. Since the temperature difference between the surface of the manikin and the cross-draft is constant, the Grashof number $(G r)$ does not change from case to case investigated and takes the value of circa $4.1 \times 10^{8}$. Based on the calculated Richardson numbers, which ranges from 2.22 to 20.87 (see Table 4.2), one should expect significant buoyancy effects for some $V_{\text {Cross-draft }}$ to $V_{\text {Face }}$ ratios. More precisely, this is strongly expected at Richardson numbers greater than 10. Although what theory says is pretty clear, it should be kept in mind that the accelerated flows are harder to disrupt compared to low speed free stream flows or quiescent environments. However, a small perturbation to the flow can cause a large change in the concentration field.

Figure 4.33 illustrates the effect of buoyancy on the exposure levels at Point 1 and Point 2 for different $V_{\text {Cross-draft }}$ to $V_{\text {Face }}$ ratios. It is evident from the figure 
that with the heated manikin the exposure trend is very similar to that predicted with unheated manikin (isothermal case). However, it is seen that the buoyancy has a notably negative influence on the exposure levels when the $V_{\text {Cross-draft }}$ to $V_{\text {Face }}$ ratio is lower than 0.133 . For this condition the calculated Richardson number is about 20.87, indicating strong effect of natural convection. For Case $\mathrm{D}\left(V_{\text {Cross-draft }} / V_{\text {Face }}=0.133\right)$ the exposure level is seen to increase $150 \%$ due to buoyancy effects. On the other hand, at higher $V_{\text {Cross-draft }}$ to $V_{\text {Face }}$ ratios where the cross-draft convection dominates the suction force, buoyancy effects are negligible. An exception seems to occur when ratio of $V_{\text {Cross-draft }}$ to $V_{\text {Face }}$ is about 0.553 (Case G). However, a closer look at Table 4.2 reveals that for this condition the Richardson number is larger than that calculated for Case F. Therefore, relatively more pronounced effect of buoyancy was already expected.

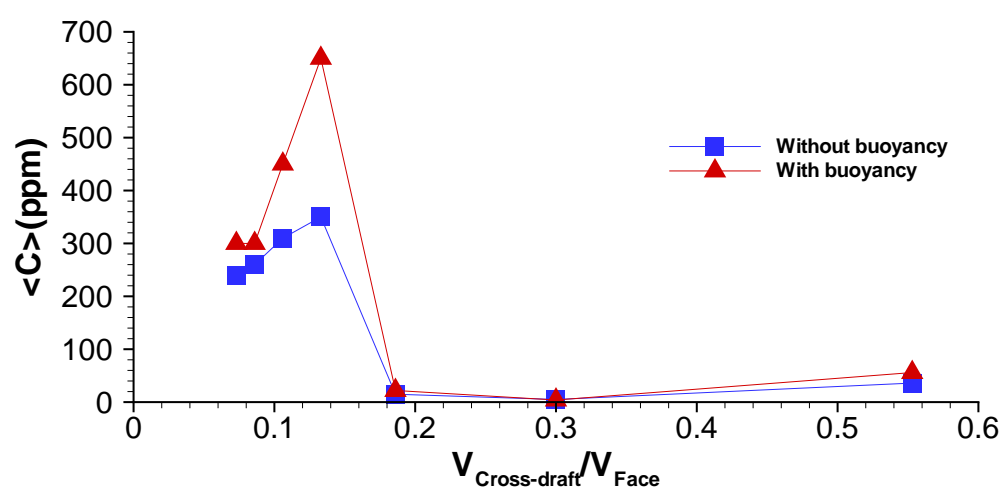

a)

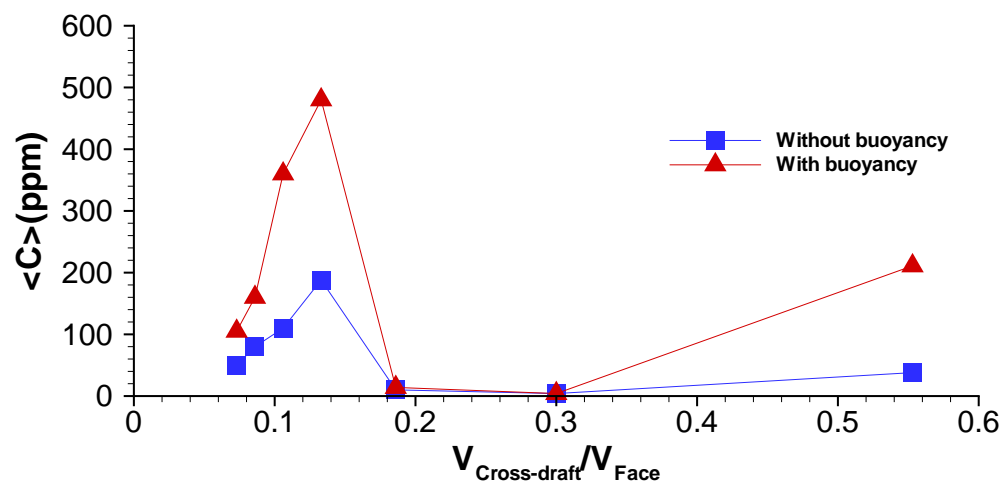

b)

Figure 4.33: Comparison of long-time-averaged concentrations from simulations with and without buoyancy a) Point 1, b) Point 2 
It is also worth discussing the heat transfer from the point of view of flow rates and flow direction as they significantly impact the temperature field in the region of interest. For this purpose, the temperature fields at low and high crossdrafts are displayed in Figure 4.34 on two planes perpendicular to each other: symmetry plane of the hood and $x y$-plane close the hood face. Comparison of Figure 4.34a and Figure 4.34b clearly shows that at lower flowrates, the fluid on the hood's central plane is heated more. On the other hand, at higher flowrates most of the heat transfer from the body to the fluid occurs in the direction of the cross-draft. Although, the heated air on the right side of the manikin is sucked into the hood, it may not flow through the central region where the contaminant is released. It is also interesting to note that the temperatures right above the contaminant source are low in both cases. Remember that there is a recirculation zone created by separation of the accelerated flow coming from under the hood. Obviously, the heat released from the worker's body can not penetrate into this recirculation zone and, consequently, buoyancy has not much impact on the transport of the contaminant at its source. However, at higher elevations, such as the shoulder and head levels, the heat transfer is relatively more pronounced. In principle, this is expected because the Grashof number increases in the vertical direction. As opposed to studies investigating impact of body heat in quiescent or low wind speed environments, where, in general, buoyancy-driven rising convection flow is observed, here the buoyancy effects manifest themselves as small perturbations to the dominant flow pattern rather than significant disruption. This is apparent from the temperature distribution around the head. Heated air is directed into the hood, not upward.

Another aspect of the problem is the impact of the buoyancy on the turbulent kinetic energy. Figure 4.35 illustrates the turbulent kinetic energy contours along with the average velocity vector field for Case $\mathrm{D}$ where the impact of buoyancy is greatest. Comparison of the two images reveals that the buoyancy perturbs the averaged flow pattern such that the vortical motions at the shoulder level are flipped around. Furthermore, turbulent kinetic energy increases due to the 


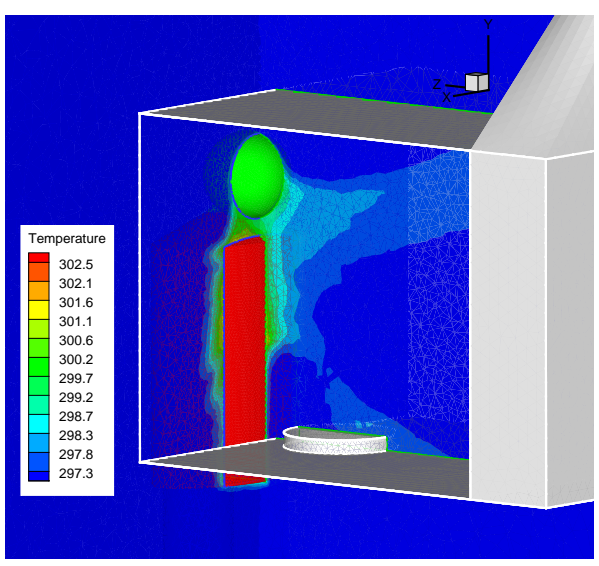

a)

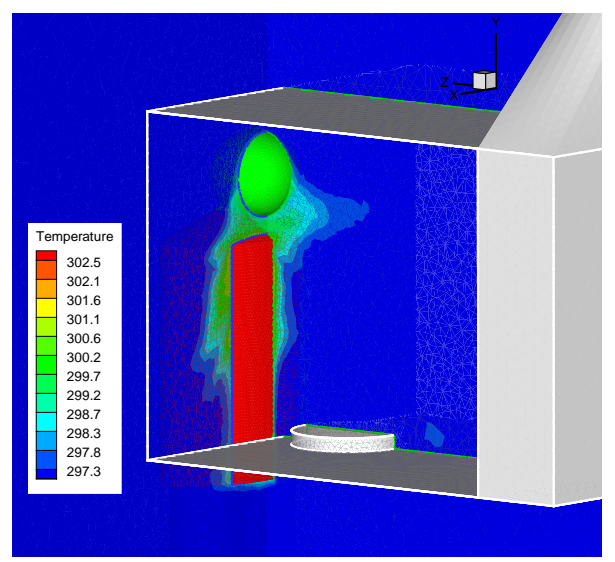

b)

Figure 4.34: Comparison of temperature fields for a) Case D and b) Case G

buoyancy. The augmented exposure level, seen in Figure 4.33, seems to be originated from the higher degree of mixing associated with increased level of turbulent kinetic energy.

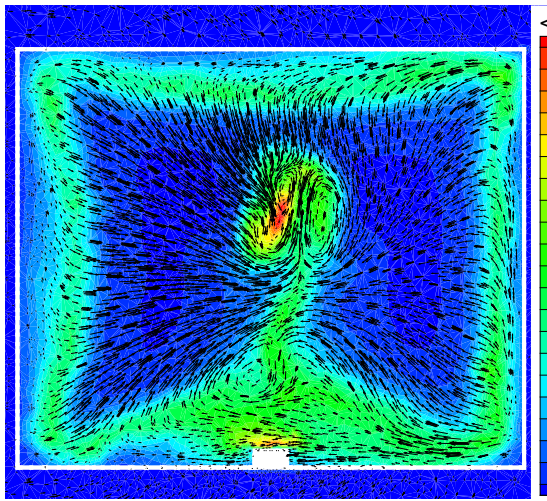

a)

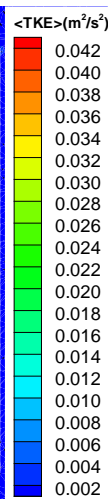

0.002

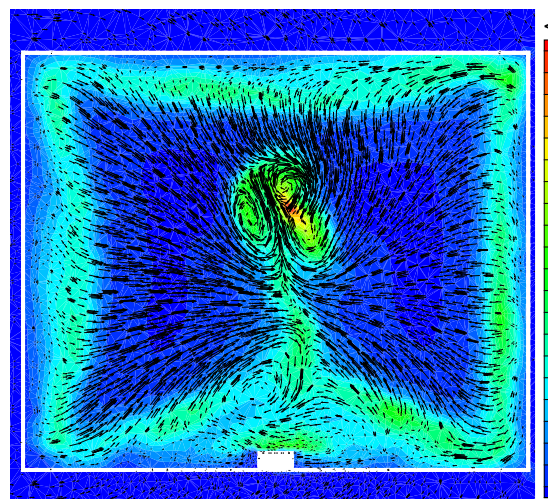

b)

Figure 4.35: Comparison of turbulent kinetic energy fields at the hood face for Case D a) without buoyancy, b) with buoyancy

Figure 4.36 demonstrates the impact of buoyancy on the instantaneous velocities for Cases D and F. Note that Cases D and F are the cases at which the exposure level is affected most and least by the buoyancy effects, respectively. Comparison of Figures $4.36 \mathrm{a}$ and $\mathrm{b}$ reveals that at low flowrate, the effect of buoyancy manifests itself through introduction of low frequency motion to the airflow. On the other hand, at high flowrate as shown in Figures 4.36c and d, the flow seems not to be affected by the buoyancy as much as the flow in Case D. 


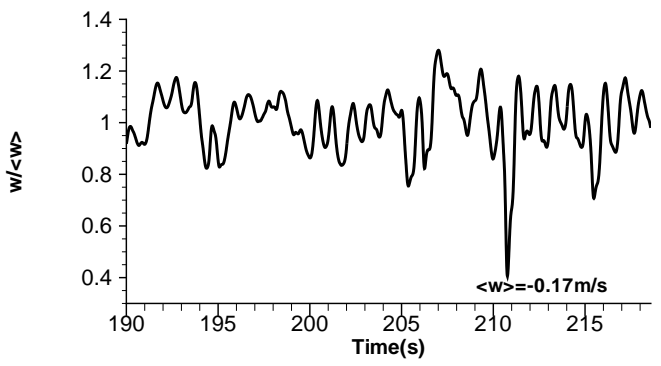

a)

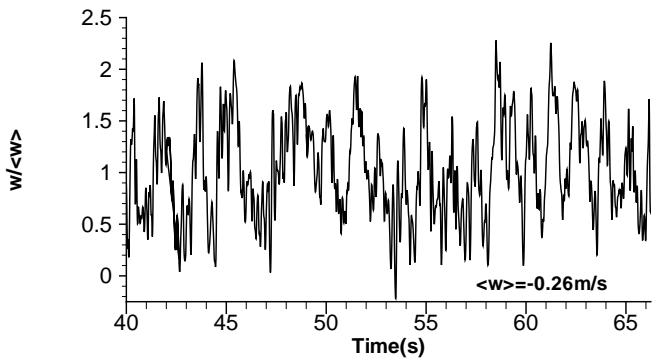

c)

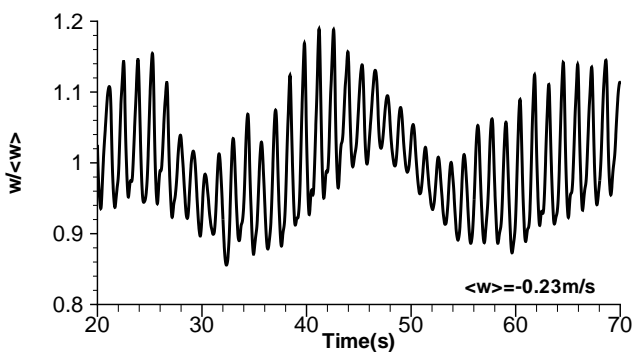

b)

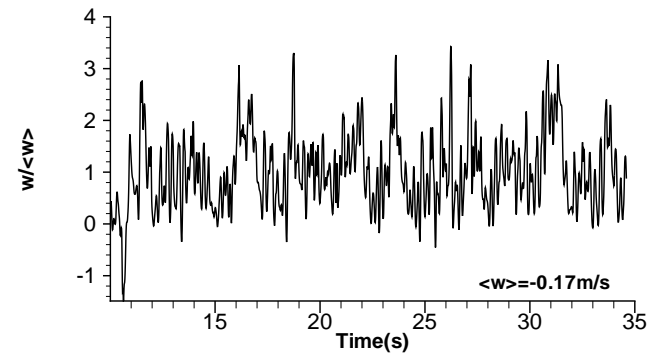

d)

Figure 4.36: Time history of $z$-velocity at Point 2 a) Case D without buoyancy, b) Case D with buoyancy, c) Case F without buoyancy, d) Case F with buoyancy

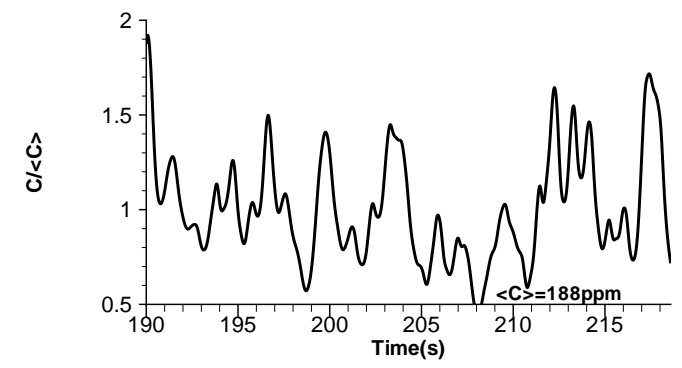

a)

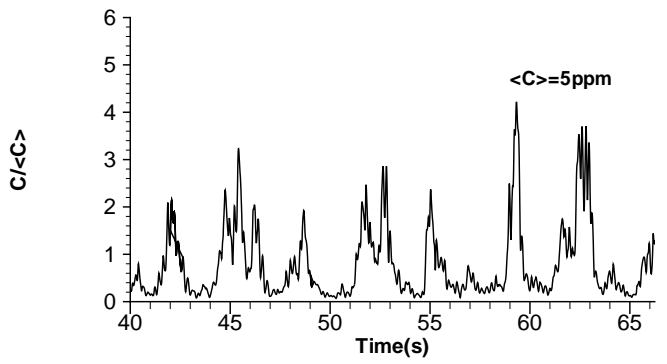

c)

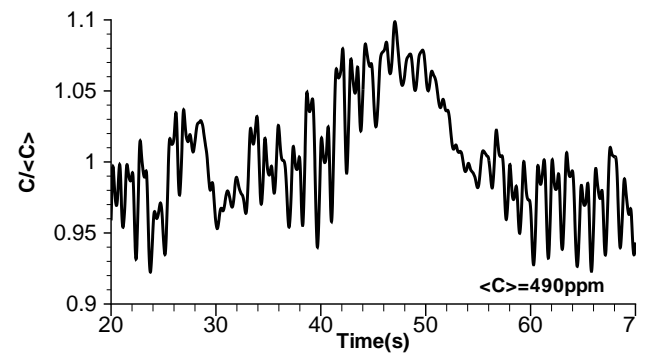

b)

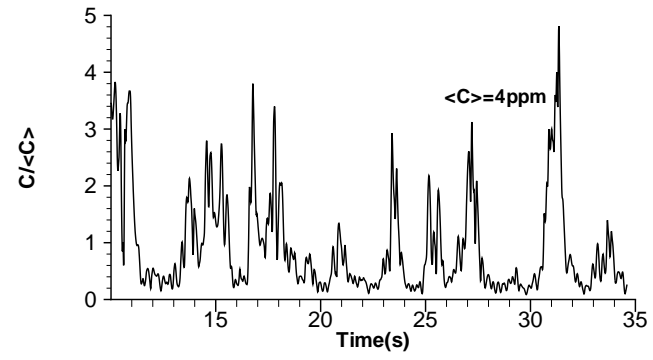

d)

Figure 4.37: Time history of concentrations at Point 2 a) Case D without buoyancy, b) Case D with buoyancy, c) Case F without buoyancy, d) Case F with buoyancy 
The effect of buoyancy on the non-dimensionalized concentration for Cases $\mathrm{D}$ and $\mathrm{F}$ is shown in Figure 4.37. It is evident from Figures 4.37a and $\mathrm{b}$ that the variability in concentrations decreases under the effect of buoyancy. This might be attributed the higher levels of mixing due to increased turbulent kinetic energy. In Case F, on the other hand, the degree of variability does not change much. This, indeed, was expected due to stronger flow compared to weak buoyancy-driven perturbations.

The effect of turbulence model on the degree of variability was tested by removing the turbulence model from the calculations and running the simulation further. As depicted in Figure 4.38, without the turbulence model, the variability of concentrations increases considerably. More precisely, the values of concentration can increase upto 15 times over time. This high degree of variability points not only to the nonlinear, dynamic behavior of the flow, but also to the diffusive nature of the RANS turbulence models. Simulation techniques utilizing less diffusive methods, such as LES, seem to be more suitable for this problem from the viewpoint of capturing the unsteadiness and the variability in the exposure levels.

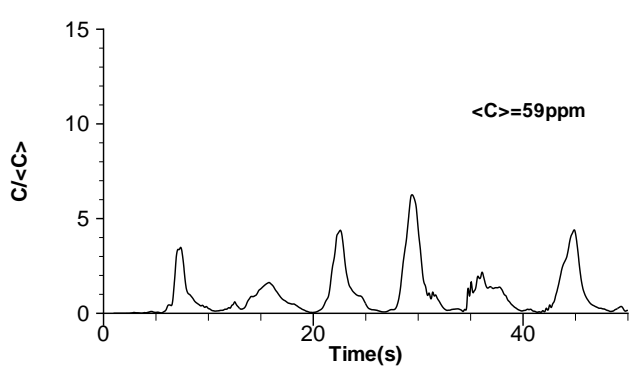

a)

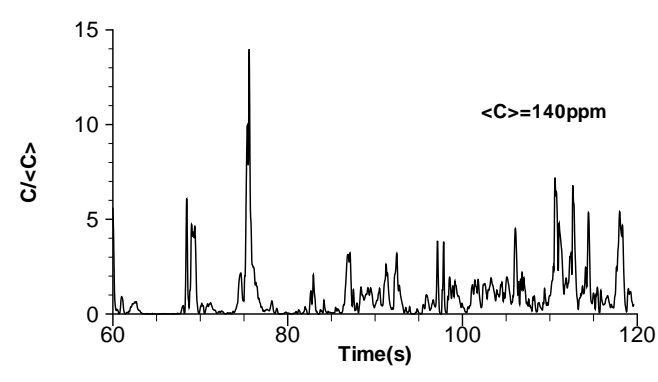

b)

Figure 4.38: Instantaneous concentrations at Point 1 under buoyancy effects (Case G), a) with RNG $k-\epsilon$ turbulence model, b) Without a turbulence model

It is also noteworthy to mention that for time series involving high degrees of variability, the period of time over which the average is taken can significantly affect the quantitative results. For example, when the average concentration is calculated using the data from $60 \mathrm{~s}$ to $120 \mathrm{~s}$, it turns out to be $140 \mathrm{ppm}$ as 
shown in Figure 4.38b. However, if the average is taken from 60s to 80s, then it becomes 105ppm. On the other hand, if it is calculated over a period from $100 \mathrm{~s}$ to $120 \mathrm{~s}$, then $206 \mathrm{ppm}$ is found. This situation, one more time, points to the need of running the simulations for much longer durations, e.g. 15min for a reliable comparison with the experiments. However, running a simulation for $15 \mathrm{~min}$ can take prohibitively long execution time with a time step of $0.001 \mathrm{~s}$.

The effect of strength of buoyancy force on the concentration level was tested for an increased temperature difference between the worker's body and the air. More specifically, the temperatures of the worker's head and the torso were increased from $300 \mathrm{~K}$ and $303 \mathrm{~K}$ to $303 \mathrm{~K}$ and $306 \mathrm{~K}$, respectively, resulting in a doubling in the temperature difference. The simulation was run only for Case $\mathrm{G}$ without using a turbulence model. The time history of the predicted concentration at Point 1 is shown in Figure 4.39. Comparison of the magnitude of long-time-averaged concentrations given Figures 4.39 and 4.38 clearly reveals that the overall exposure level increases with increasing buoyancy force. This is an expected result since the stronger the buoyancy driven upward flow, the more contaminant is transported to the worker's breathing zone. In terms of the degree of variability, stronger buoyancy force does seem to have generally an augmenting effect on the variability of the concentration level. This is clear when Figures 4.39 and 4.38 are compared in terms of concentration magnitudes.

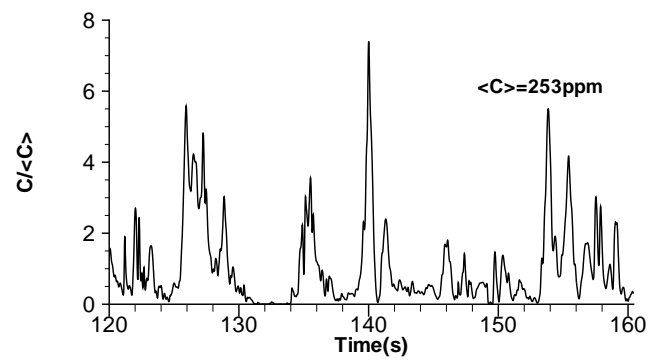

Figure 4.39: Instantaneous concentrations at Point 1 under buoyancy effects for the increased temperature difference (Case G) 


\subsubsection{Effect of Body Shape}

Using simple shapes to represent a human body is commonly employed in numerical simulations and also in most experimental studies due in part to high computational cost associated with the very fine mesh required to resolve the complex shape, due in part to considerable change in body shape from person to person. However, it should be clarified whether this approximation hinders some phenomena that can affect the conclusions of a study.

For this purpose, in the present study, the exact shape of the anthropometrically-scaled, complex manikin used in the experiments was incorporated into numerical simulations. In this case, the manikin was in working position (i.e., his arms are reaching into the hood), unlike the simple manikin which represents a worker with arms resting on the sides. In an attempt to incorporate the complex manikin into simulations, first a CAD model of the manikin was generated using a three-dimensional laser scanner and an appropriate reverse engineering software called 3DReshaper ${ }^{\circledR}$, then it was imported into GAMBIT in order to create the computational mesh. Details of the CAD model generation can be found in the Appendix A. Due to the complex shape of the manikin, a fine mesh consisting of 2,089,971 tetrahedral cells were generated. Figure 4.40 depicts the mesh structure on the manikin and hood surface. The simulation was run only for Case D, due to excessive computational cost.

Figure 4.41 illustrates the predicted flow and concentration fields at the hood face for both complex and simple manikins. Comparison of the two contour plots indicates that the flow fields are similar in that vortices at the shoulder level are present in both predictions. However, the large vortex at the waist level seems to be prevented by the complex manikin's arms. The transport of contaminants to the manikin's face seems unaffected from this. It should be kept in mind that, in practice worker's arm movements can enhance the turbulence which, in turn, may lead to higher levels of exposure. 


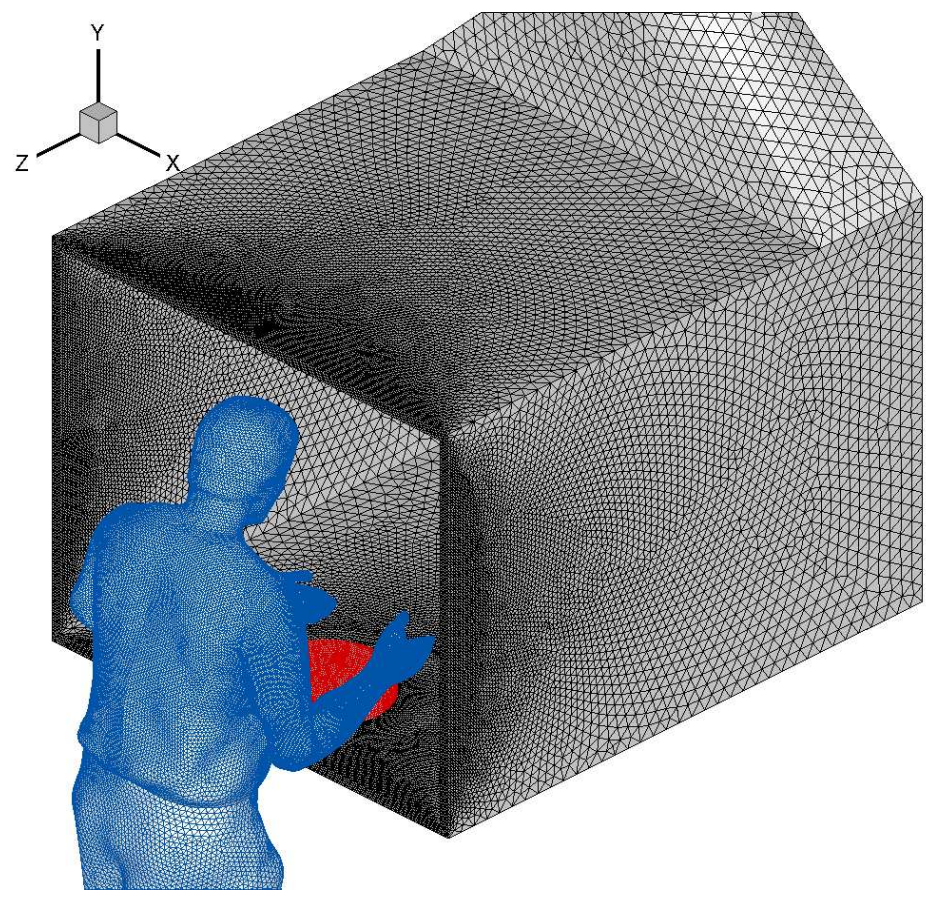

Figure 4.40: Mesh structure on the manikin and hood surface

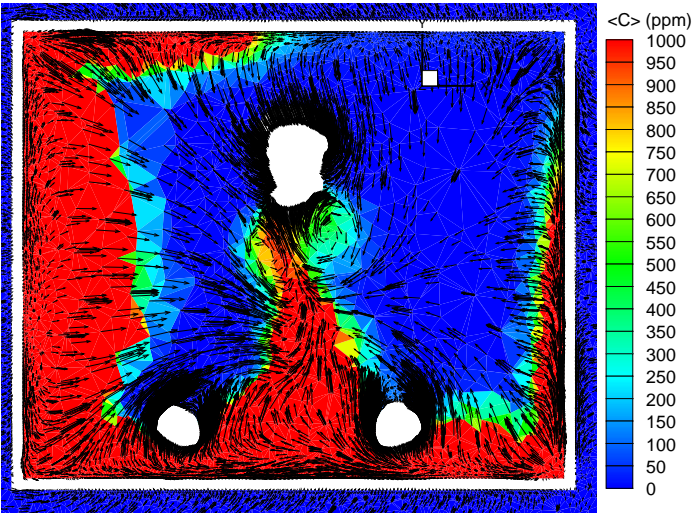

a)

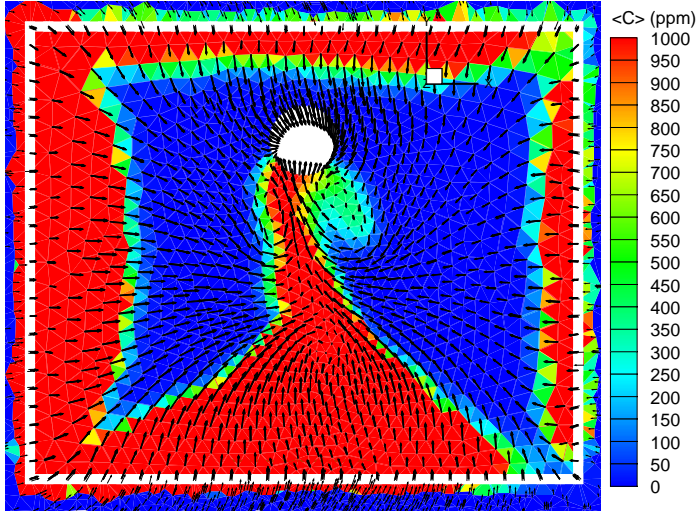

b)

Figure 4.41: Predicted velocity and concentration fields at the hood face for Case D a) Complex manikin, b) Simple manikin

Figure 4.42 shows the concentration levels on the surfaces of both manikins. Comparison of Figure $4.42 \mathrm{a}$ and Figure $4.42 \mathrm{~b}$ reveal that the concentration level on the face is affected by the shape of the manikin. From Figure $4.42 \mathrm{a}$, it is seen that the high concentration region, which corresponds to red color, starts from the belly level and reaches the chin, which, to some extent, limits the rise of contaminants to the manikin's face. More clearly, high concentrations 
remain mostly under the chin. On the other hand, due to smooth, spherical representation of the head, the high concentrations reach cover a wider region on the simple manikin's face. This can also be seen in Figure 4.43, where the flow and concentration fields are shown on the vertical symmetry plane. Also from the figure it is seen that there is stonger upward flow in front of the complex manikin. However, the predicted concentration levels at Point 1 and Point 2 for both manikins are comparable, as shown in Figure 4.44. This may also indicate that the fine grid used for simple manikin is sufficiently fine.

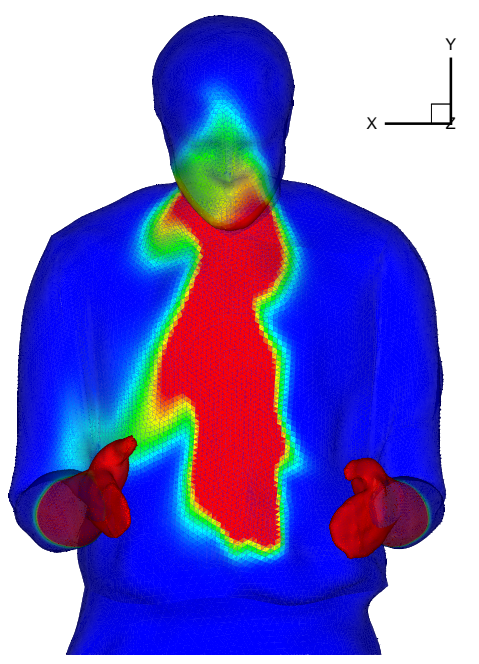

a)

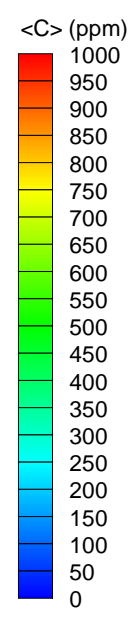

Figure 4.42: Predicted velocity and concentration fields on the the manikin's surface for Case D a) Complex manikin, b) Simple manikin

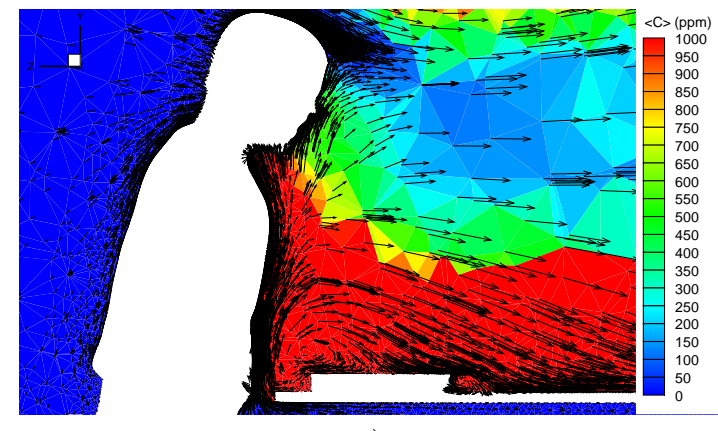

a)

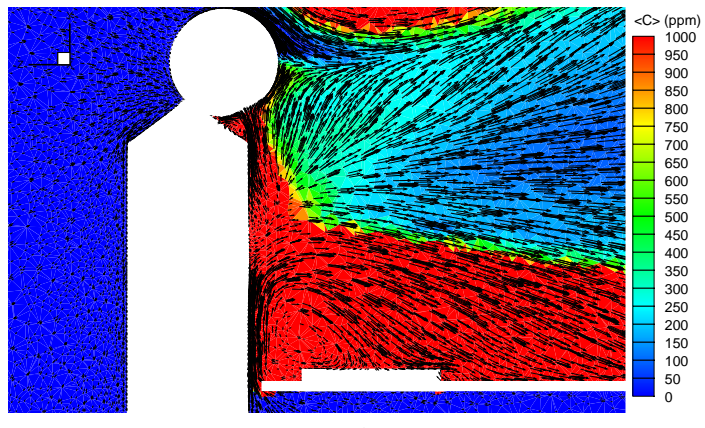

b)

Figure 4.43: Predicted velocity and concentration fields on the vertical symmetry plane for Case D a) Complex manikin, b) Simple manikin 


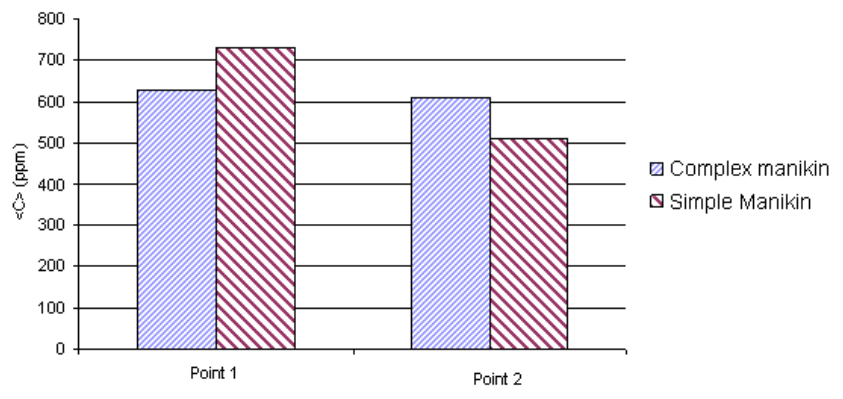

Figure 4.44: Comparison of concentration levels for Case D with complex and simple manikins

Due to its irregular topology the complex manikin is expected to be more prone to flow separation which may result in some unsteady flow structures in his wake. However, the simulations with complex manikin resulted in steady flow. Figures 4.45 and 4.46 show the time history of $z$-velocity and concentration levels, respectively, at Point 1 and Point 2. As can be seen from the figures the initial transients die out after around 100s and only minor fluctuations are sustained. Concentration levels seem to follow the trend in the flow.

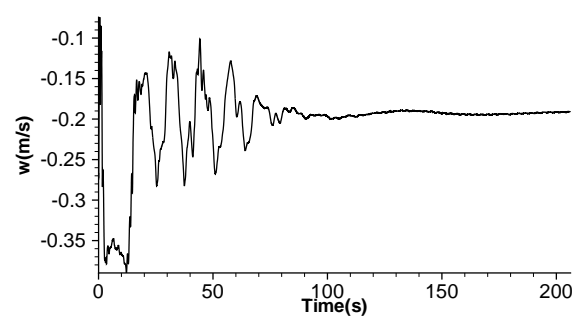

a)

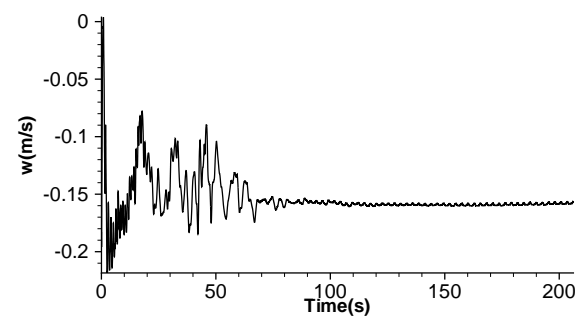

b)

Figure 4.45: Time history of $z$-velocity for Case D a) Point 1, b) Point 2

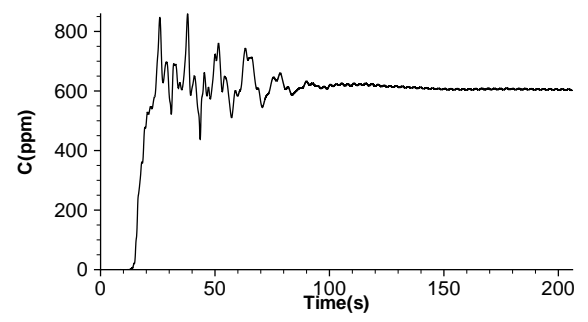

a)

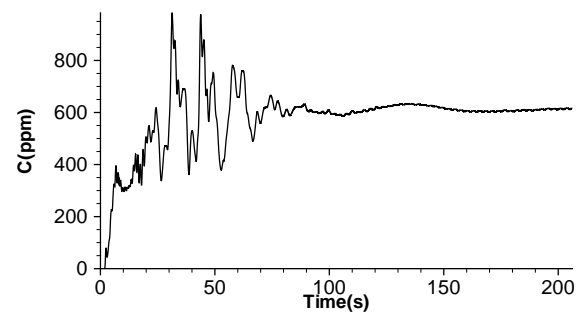

b)

Figure 4.46: Time history of concentration for Case D a) Point 1, b) Point 2 


\section{Chapter 5}

\section{Parallel Implementation of the DREAM}

In general, CFD simulations are very demanding in terms of computational power and memory requirements. This is especially true when simulating large problems which require resolution of a wide range of length and time scales and consideration of other phenomena such as heat transfer, mass transfer, and chemical reactions. In fact, one of the major limitations of modern CFD codes, despite of the exponential increase in the speed and memory of the computers in recent years, is the size of the problems that could be handled on a single processor. In some cases, even though a large problem could be run on a single processor, it might take excessive amount of time rendering it impractical. High

Performance Computing (HPC) or parallel processing is generally employed to distribute the work and memory load across a cluster of computers allowing rendering of large problems that do not or barely fit into memory of a single processor. In an attempt to make the DREAM code a usable computer code for solving larger problems, a parallel algorithm based on Domain Decomposition Technique (DDT) has been conceived. In what follows, implementation of the parallel algorithm, structure of the parallel code and its application to several test cases for validation and testing its performance are presented. 


\subsection{Parallel Algorithm and Code Structure}

In this work, a distributed computing form of parallelism in conjunction with the well known Domain Decomposition Technique (DDT) and overlapping grid approach is employed. The entire computational domain along with the grid is partitioned into as many sub-domains as the number of processors assigned for parallel processing of the problem at hand. This leads to sub-domains with matching grids at the interfaces (virtual boundaries) between neighboring subdomains (see Figure 5.1). Each sub-domain is assigned to a processor and in each processor, including the root (master), the same calculations are performed.

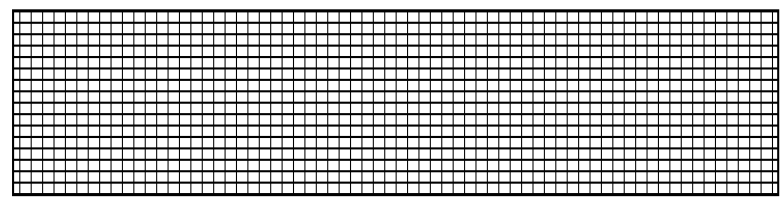

Domain

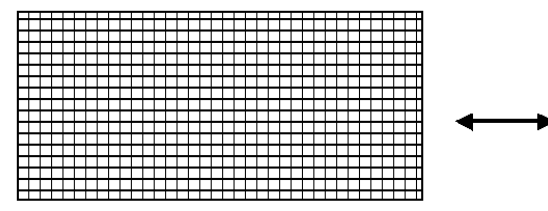

Sub-domain

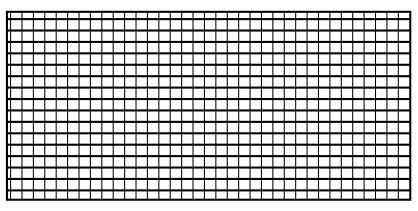

Sub-domain

Figure 5.1: Domain decomposition.

The flow chart of the parallel DREAM, which is called DREAM_P hereafter, is presented in Figure 5.2. As can be seen from the figure, leaving the fact that MPI [56] initialization and finalization, and output writing carried out by the root processor only, the presented algorithm can be categorized as Single Program Multiple Data (SPMD). It is worth noting that since iterative solvers are incorporated into DREAM, the parallelization is not performed at the solver level (i.e. the linear system solver) but at the discretization level. Application of DDT to direct solvers which requires reconstruction of the solution matrix is a hot topic in parallelization. However, in the present study, each processor computes the coefficients for the linear system to be solved for each variable in the assigned sub-domain and then solves the system. Therefore, in the 
calculation of the coefficients of the solution matrix for the grid points on the virtual boundaries, data from the old time step, communicated by the processors solving for respective adjacent domains is used. Strictly speaking, an overall iteration is needed to ensure that solutions obtained for individual sub-domains are continuous across the internal domain boundaries (i.e. global convergence of the solution in the whole domain) at every time iteration. However, as long as the time step is small enough to ensure that quantities do not change significantly from one time step to another, this approach, i.e. without overall iterations within each time step, works just fine without compromising solution accuracy. Since parallelization of the DREAM is part of a larger project aiming at LES of turbulent flows, which necessitates very small time steps, the applied methodology constitutes a practical solution to the problem.

As it is seen from the flowchart given in Figure 5.2, after calculating the tentative velocity and the corresponding pressure and scalar fields within a time step, three-dimensional data $\left(u_{i}, \phi\right.$, and $\left.p\right)$ exchange is performed between the sub-domains (processors). This data exchange is carried out via the ghost cells spared for overlapping. The overlapping system of grids are advantageous since it offers a smooth transformation for each sub-domain and simplifies the grid generation, especially in complex geometric domains [57]. In order to maintain the spatial accuracy at the virtual boundaries, in this study, two ghost cells were used which enable the use of same second and third order spatial discretization stencils as for the inner grid nodes. If needed, utilization of higher degrees of overlapping is a straightforward task. Although the computations are carried out in three-dimensions, for simplicity of explanation, the data transfer paradigm on a two-dimensional backward staggered grid is depicted in Figure 5.3. Threedimensional data transfer is performed in the same fashion.

In Figure 5.3, the bold lines indicate the borders of the computational subdomains, while the cells outside of the computational sub-domains are the ghost cells. As mentioned earlier, the exchanged (old time step) data at the virtual boundaries is directly stored in the respective variable arrays for that domain 


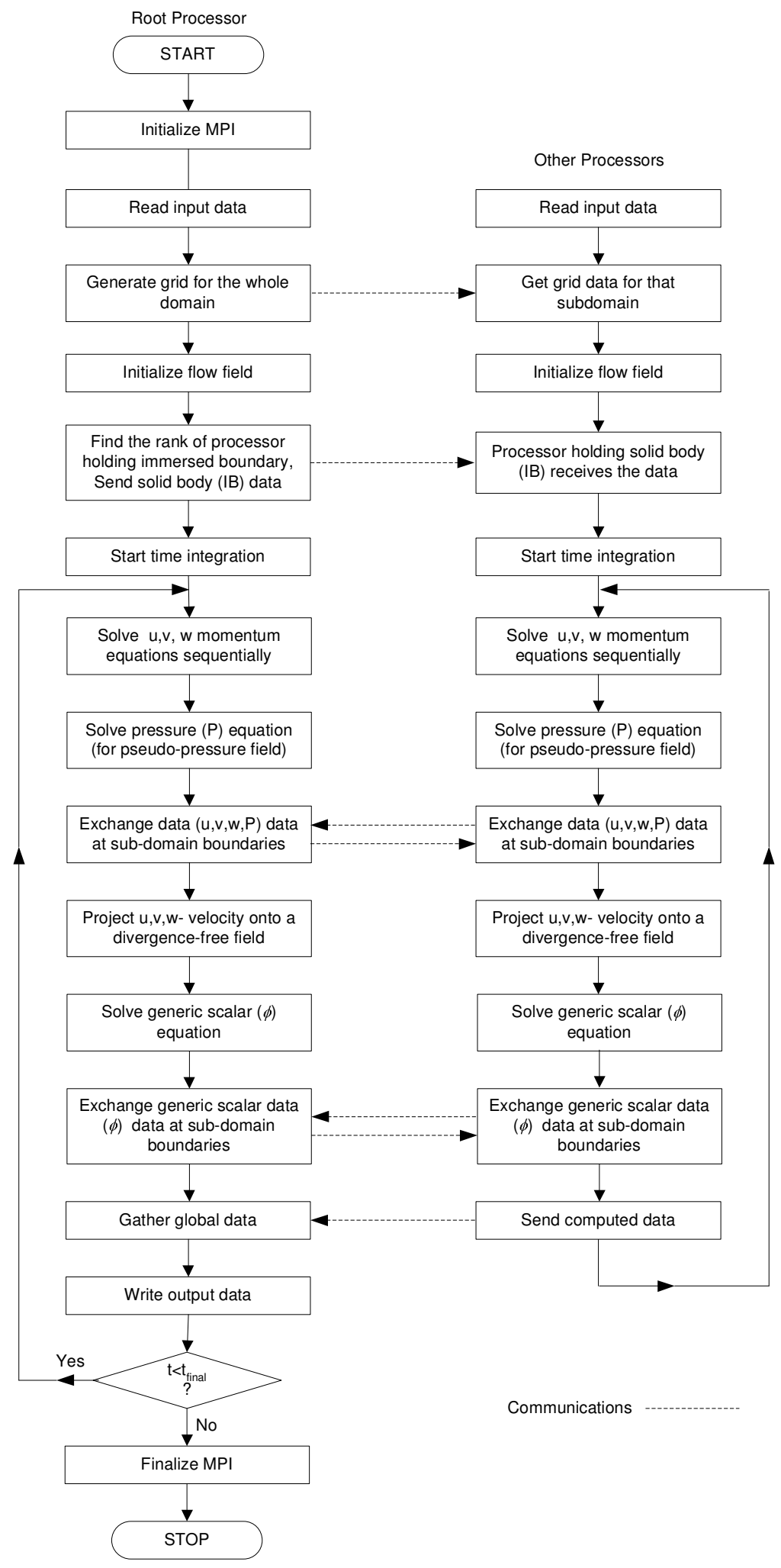

Figure 5.2: Flowchart of the DREAM_P 
and is subsequently used in the explicitly evaluated terms and also in the linear system solver. To be precise, at the virtual boundaries which become the boundaries for the internal domains, instead of applying boundary conditions by appropriately altering the coefficients of the linear system, the boundary data is stored in the respective variable array so that it is directly accessed by the solver and is used in the iterative solution process for the interior nodes. Thus no special treatment (other than data exchange) is required for the virtual boundaries with overlap, and specific boundary conditions are applied only at the actual boundaries of the overall domain.

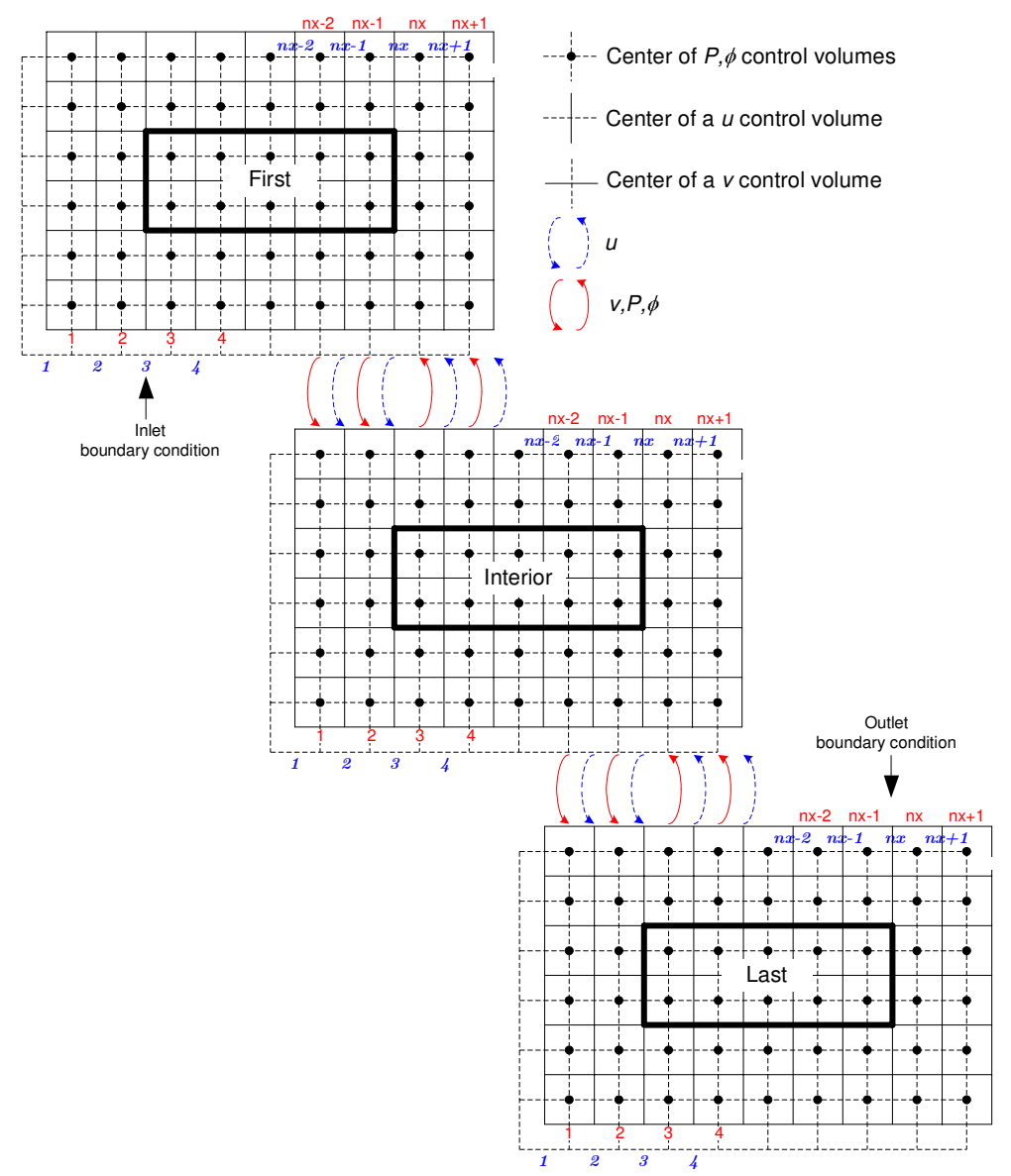

Figure 5.3: Two point data exchange with four points overlap paradigm

In the present study, the entire computational domain is decomposed into sub-domains only in the axial direction and except for Test Case III which will be introduced subsequently, the sub-domains of identical sizes were used 
to alleviate the need for load balancing. The computations were performed with up to 16 processors belonging to a 64 processors ( 8 dual quad-core Xenon 2.66 Ghz processors) Linux cluster linked with Gigabit ethernet network and MPI library [56] for message passing. Each processor is accommodated with 2MB of cache and $2 \mathrm{~GB}$ of RAM resulting in $128 \mathrm{~GB}$ in total.

The performance gain achieved by running a code in parallel is evaluated in terms of speed-up, $S_{p}$, and efficiency, $E_{p}$. Speed-up is defined as the ratio between the execution times of sequential code, $T_{1}$, and parallel code on $p$ processors, $T_{p},\left(S_{p}=T_{1} / T_{p}\right)$. Efficiency of the parallel processing is defined as the ratio between the speed-up achieved on $p$ processors and the number of processors $p\left(E_{p}=S_{p} / p\right)$.

\subsection{Verification of Parallel Algorithm}

The accuracy and performance of DREAM_P was tested for three test cases, namely, developing flow between parallel plates, lid driven cavity flow and turbulent flow over a square cylinder. The first two cases are twodimensional problems whereas Test Case III is three-dimensional. In all cases, the convergence criterion within a time step; the ratio of the residual at an iteration to the residual of the first iteration of the same time step, was set to $10^{-8}$. As to the discretization of convective terms, QUICK scheme was used in all cases.

\subsubsection{Test Case I: Developing Flow between Parallel Plates}

The goal of applying DREAM_P to simulation of fairly simple, parabolic flow between parallel plates is to assess its temporal accuracy. For this purpose, a computational domain measuring $1 \mathrm{~m} \times 0.1 \mathrm{~m}$ in the $x$ - and $y$-directions, respectively, were selected. The computational domain is schematically depicted in Figure 5.4. The flow is laminar with a Reynolds number of 500, based on the channel height $(H=0.1 \mathrm{~m})$, uniform inlet velocity $\left(U_{0}=0.05 \mathrm{~m} / \mathrm{s}\right)$ and kinematic viscosity $\left(\nu=10^{-5} \mathrm{~m}^{2} / \mathrm{s}\right)$ 


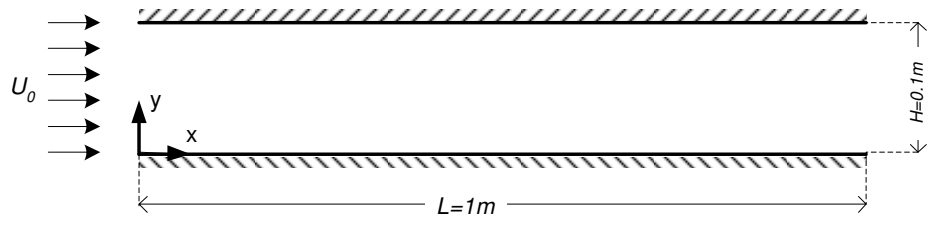

Figure 5.4: Schematic representation of flow between parallel plates

A uniformly distributed mesh consisting of $100 \times 20$ cells, in the $x-$ and $y$-directions, respectively was used. For parallel processing the computational domain and the grid was decomposed into 4 sub-domains in the axial direction with identical domain sizes and grids. The flow field was initialized with the inlet velocity, $U_{0}$. With a constant timestep $(\Delta t)$ of $0.001 \mathrm{~s}$ simulations were conducted for $40 \mathrm{~s}$ which corresponds to two flow-through-times $\left(t_{F T}=L / U_{0}=20 \mathrm{~s}\right)$. As to the boundary conditions, no slip boundary conditions were imposed at the top and bottom walls. The outflow boundary condition, ensuring the global mass conservation was invoked at the outlet. Computations were performed using fully explicit scheme. For time integration Adams-Bashforth scheme was employed. The predictive accuracy of the parallel DREAM code was assessed by comparing transient results with the ones obtained from sequential DREAM_P and FLUENT runs.

Figures 5.5 and 5.6 shows the time variation of the streamwise velocity and pressure profiles along the horizontal centerline of the channel, respectively. It is evident from the figure that, the transient results from sequential and parallel versions of the DREAM code collapse on a single curve which compare very well with the FLUENT's predictions. Similarly, as shown in Figure 5.7, the transient $u$-velocity profiles along the height of the domain in the middle of the channel are in very good agreement with those predicted by the sequential DREAM and the FLUENT. Since FLUENT is an extensively validated and verified commercial software, the observed agreement may serve as an independent, but indirect verification of the temporal accuracy of both the sequential and parallel versions of DREAM. Moreover, it is felicific noting that, without the overall iterations within a time step for the global convergence of the solution in the whole domain, 
substantial temporal accuracy was achieved even with a moderate time step. However, one should keep in mind that since the pressure is not solved globally over the entire domain, depending on the case simulated, it can take a few time steps in order a reasonable pressure field to be established after initiating the simulation.

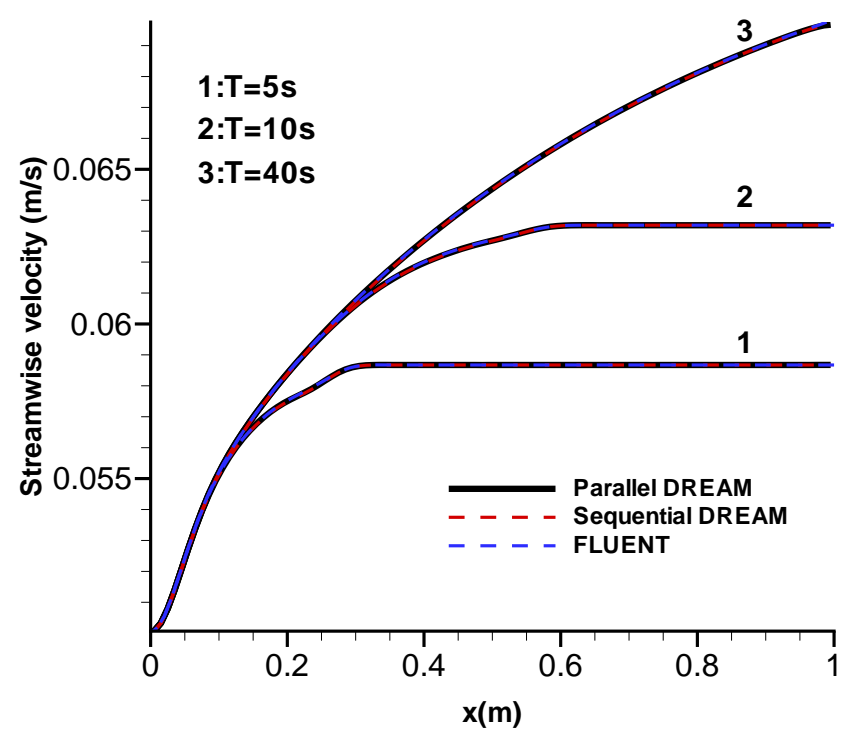

Figure 5.5: Time variation of streamwise velocity along the channel length at the centerline.

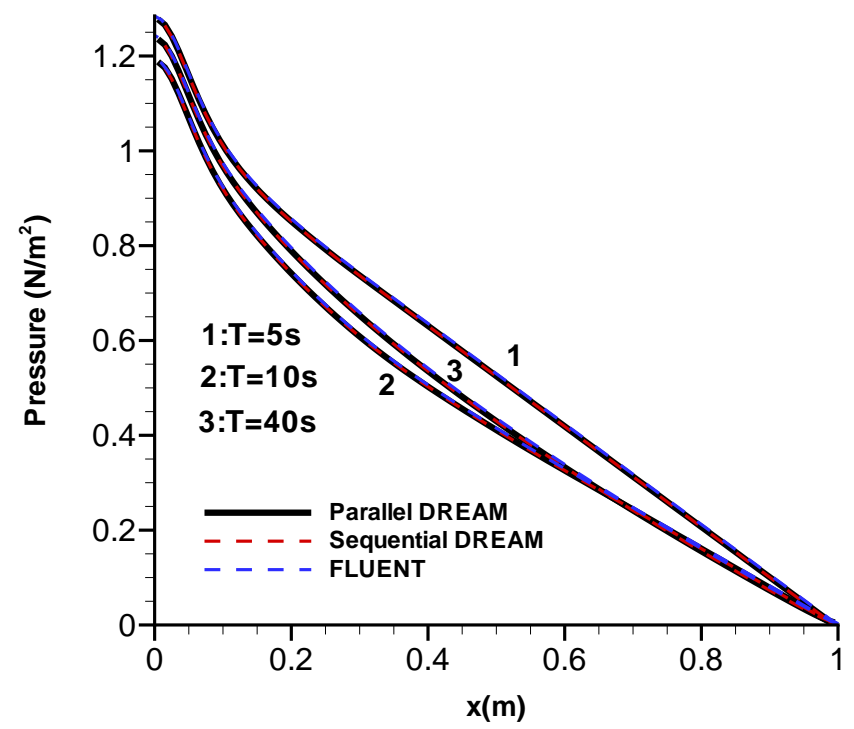

Figure 5.6: Time variation of pressure along the channel length at the centerline. 


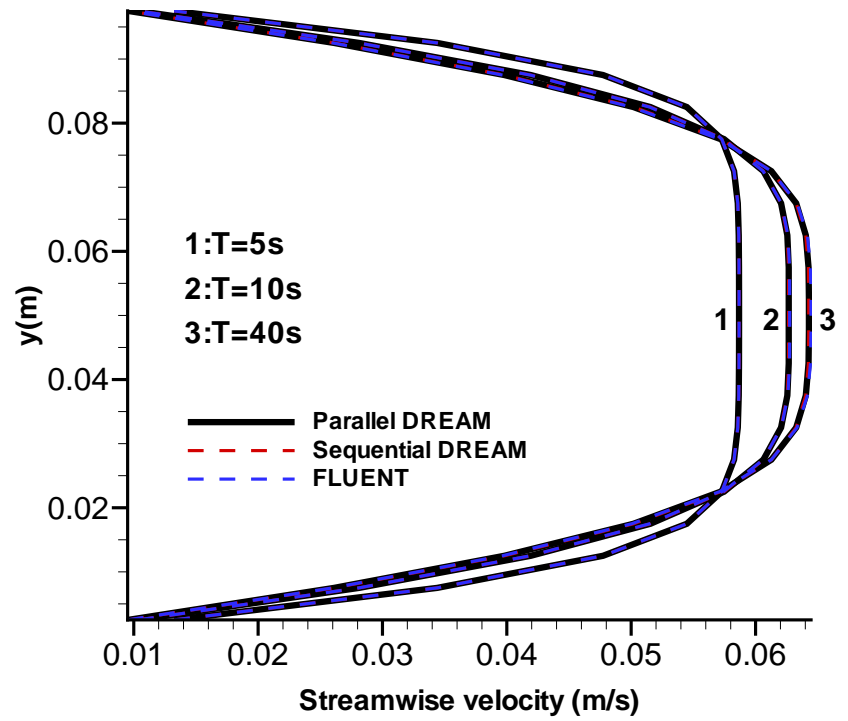

Figure 5.7: Time variation of streamwise velocity along the channel height at the centerline.

\subsubsection{Test Case II: Lid-Driven Cavity Flow}

Having validated the predictive accuracy of the parallel code for a simple parabolic flow, the focus has moved to a relatively more complex problem; simulation of lid driven cavity flow. Despite its geometrical simplicity, lid driven cavity flow is one of the most popular test problems used in validation of the CFD codes due to the rich flow physics (e.g. counter rotating recirculation zones) it retains. In the present study, the governing equations were solved for a Reynolds number $(R e=u L / \nu)$ of 100 , for which the physical system along with the characteristic primary and secondary recirculation zones (vortices), denoted by $\mathrm{BL}$ and BR, are schematically shown in Figure 5.8. As shown in the figure, the velocity of the top wall is $1 \mathrm{~m} / \mathrm{s}$, whereas all other walls (left, right, bottom) are stationary.

The parallel computations were performed with 2, 4, 8 and 16 processors (sub-domains) and the results obtained were compared with the highly accurate benchmark solutions [58] found in the literature as well as with the outputs of the sequential DREAM code. Furthermore, a performance test in terms of 


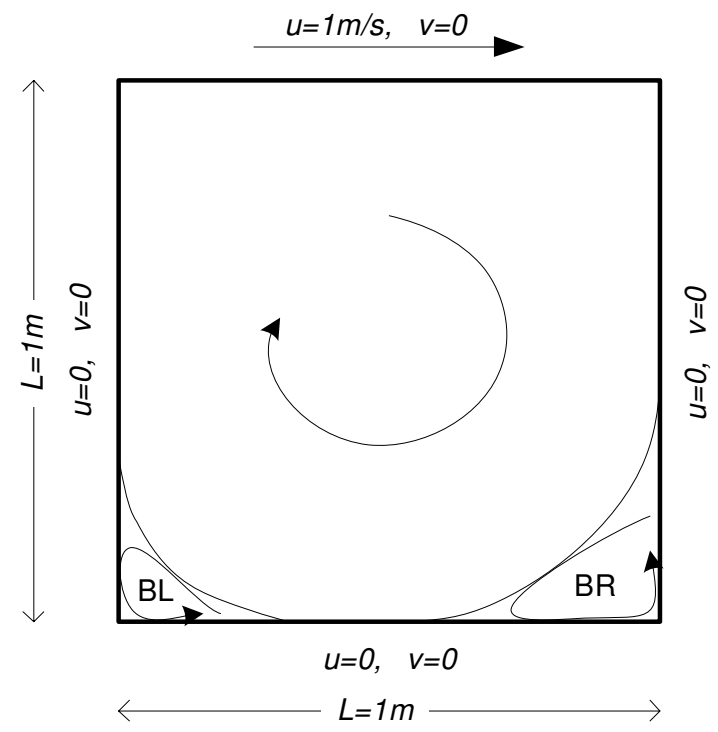

Figure 5.8: Schematic view of the physical system along with anticipated vorticies. BL: Bottom Left, BR: Bottom right

speed-up and efficiency is performed. To conform with the benchmark study of Ghia et al. [58], almost identical grids constituting of $128 \times 128$ and $256 \times 256$ cells in $x$ - and $y$-directions, respectively, were used. The numerical scheme used is the semi-implicit Crank-Nicholson scheme. The flow field was initialized from a stagnant condition and simulations were run until steady state (40s) with a constant time step of $0.001 \mathrm{~s}$.

Figure 5.9 displays the comparison of the steady-state streamfunction contours from sequential and parallel runs utilizing 4 processors and $128 \times 128$ grid. As can be seen from the figure, the flow field predictions from parallel and sequential codes are in very good agreement. Although not shown here, the calculated streamfunctions also agree very well with those reported by Ghia et al. [58] and by Carvalho and Palma [59], who revisited the same problem for the validation of their parallel CFD code. Nonetheless, the success of benchmarking can be deduced from Figure 5.10, where the $u$ - and $v$-velocity profiles along the vertical and horizontal lines through the geometric center of the computational domain are compared with the benchmark solution of Ghia et al. [58]. The 
excellent agreement seen in the velocity profiles verify the accuracy of the parallel DREAM code for recirculating flows.

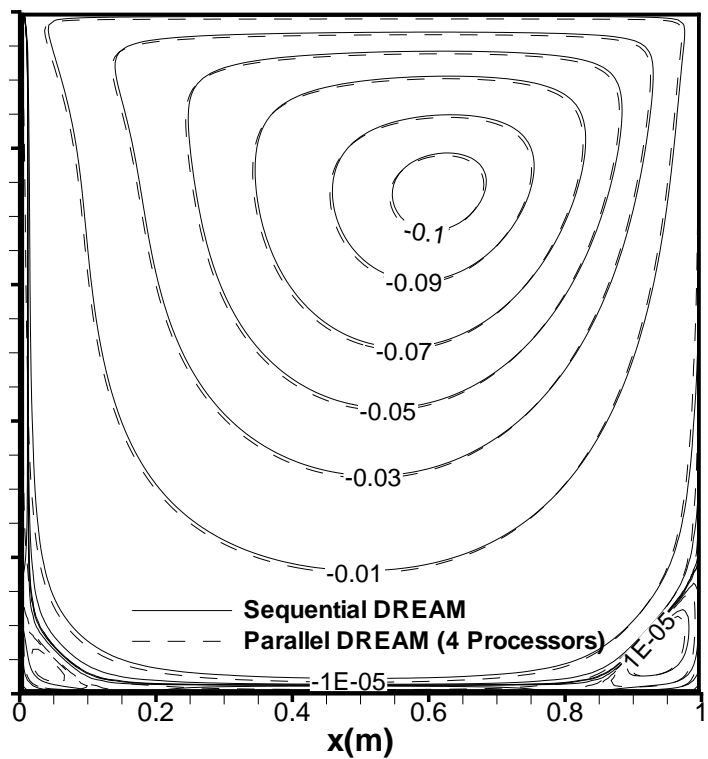

Figure 5.9: Streamfunction contours obtained from the sequential and parallel runs with 4 processors

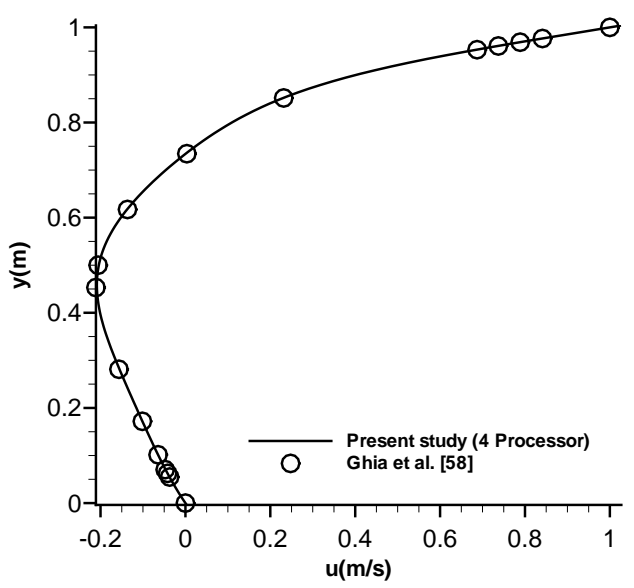

a)

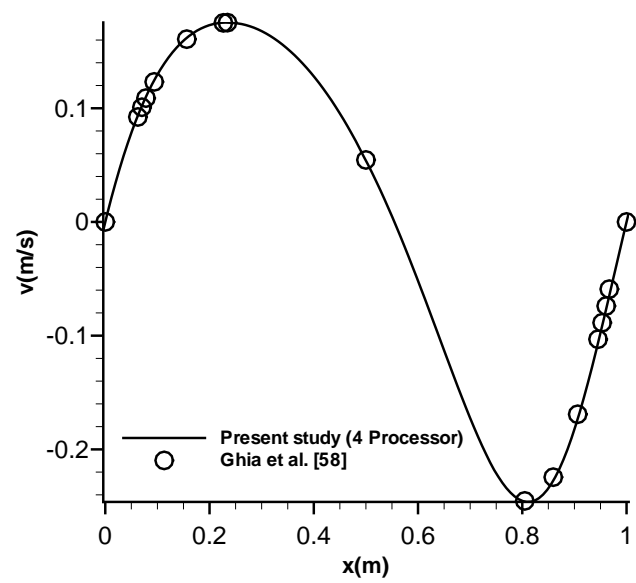

b)

Figure 5.10: Comparison of velocity profiles obtained from sequential and parallel runs, a) $u$-velocity, b) $v$-velocity

In an attempt to test the gain due to parallelization, the performance metrics; speed-up and efficiency, achieved for the utilized grid sizes were calculated and 
presented in Figures 5.11 and 5.12., respectively. As can be seen from the figures, for all grids superlinear speed-ups (efficiencies in excess of 100\%) were achieved in parallel runs. At a first glance, superlinear speed-up seems surprising or even suspicious. Principally, one would usually expect only sublinear speed-up due to communication and synchronization overheads. However, superlinear speed-ups are not so uncommon in the literature $[60,61]$. In fact, the performance of a CFD code critically depends on memory fetch. If appropriate cache load is achieved, the average memory access time is reduced leading to superlinear speed-ups due to cache's much (5-10 times) lower latency than that of main memory (RAM). Indeed, superlinear speed-ups may be indication of good scalability of the code.

It makes sense that as the number of grid points decreases with increasing number of processors, the cache miss ratio decreases meaning fewer data is fetched from RAM. However, one should be aware of the fact that this linear speed-up, of course, do not last forever. As more processors are used, the time needed for loading data into cache reduces to a minimum and remain stable while the time spend for massage passing increases. The speed-up will quickly become sublinear. Indeed, a hint of this trend is seen in Figure 5.12. When the number of processors used are doubled from 8 to 16, efficiency decreases, especially for $256 \times 256$ grid, indicating that message passing time start to limit the execution speed.

In order to ensure that this postulation is correct, the problem is scaled up by adding another dimension in the spanwise $(z-)$ direction. Simulations were performed for a set of grid points orderly increased in the z-direction. Symmetry boundary conditions were imposed in the spanwise direction. The speed-ups and efficiencies achieved were illustrated in Figures 5.13 and 5.14. From the figure it is obvious that with increasing grid points in the spanwise direction, meaning increasing communication overhead, the speed-ups decrease from superlinear to sublinear, and correspondingly, efficiencies to lower values. On the whole, the parallel DREAM code constitutes a major improvement to the serial code. 


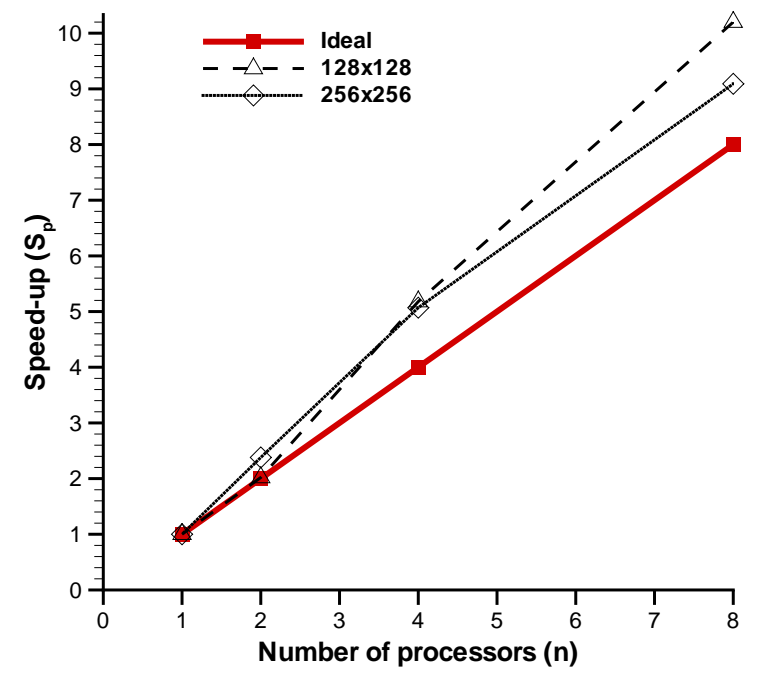

Figure 5.11: Speed-up vs. number of processors for two grids

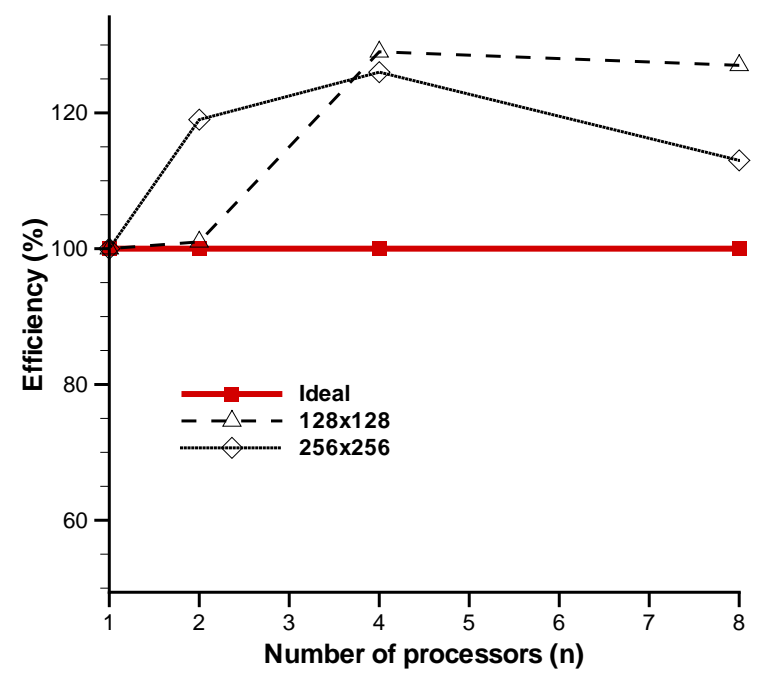

Figure 5.12: Efficiency vs. number of processors for two grids 


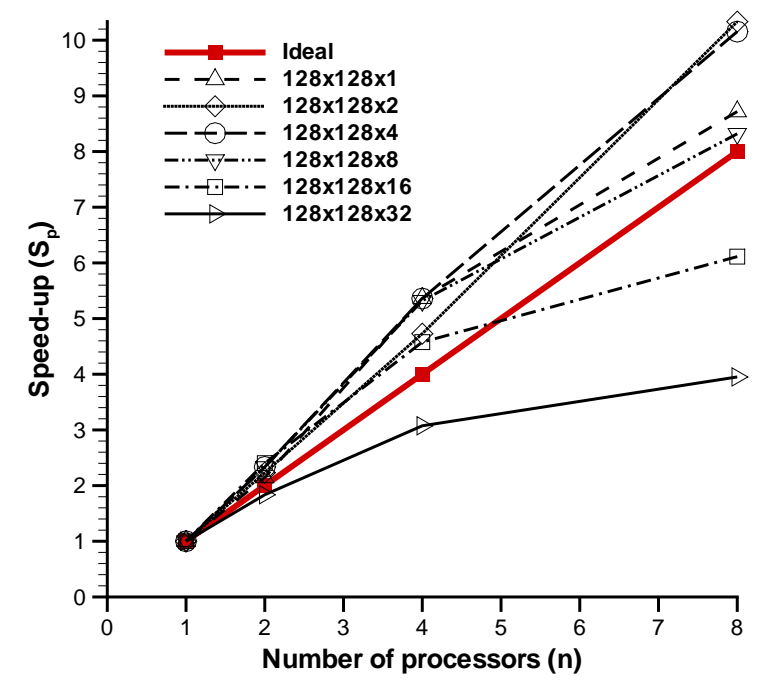

Figure 5.13: Speed-up vs. number of processors for different grids

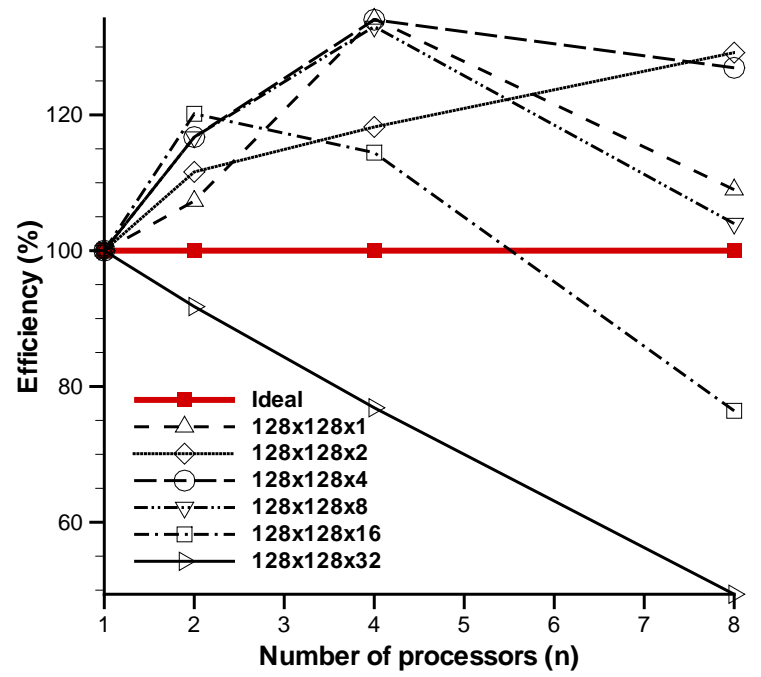

Figure 5.14: Efficiency vs. number of processors for different grids 


\subsubsection{Test Case III: Flow Over a Square Cylinder}

The last test case chosen for verification of the parallel code is LES of flow over a square cylinder for a configuration proposed by Lyn et al. [62]. The current problem is more challenging from previous test cases in that it is turbulent and involves complex flow phenomena, such as separation and reattachment, multiple separations with partial reattachment, recirculation, unsteady vortex shedding, curved shear layers, bimodal flow behavior, transition from laminar to turbulent flow, high turbulence level and three-dimensional large-scale structures [63].

The situation investigated by Lyn et al. [62], which will be described subsequently, has become a benchmark case for numerical investigations of the flow past square cylinder, within the LES framework. Two LES workshops, aimed at exploring the LES capability in reproducing this experiment's results, were held in 1995 and in 1997. Results from the workshops are published in [64-67]. Recently, Hu [49] validated the sequential DREAM code for LES of the same test problem using Implicit Turbulence Modeling (ITM) and dynamic eddy viscosity SGS models. In the present study, the computations carried out by $\mathrm{Hu}$ [49] are repeated using parallel version of the DREAM code. The results obtained are compared with sequential DREAM code results and with the solutions available in the literature.

The geometry used for the simulation is shown in Figure 5.15. To avoid ambiguity, it has been assumed that the origin of the coordinates is at the center of the cylinder. All the lengths are scaled with the side length of the square cylinder, $D$. The calculation domain extends $4.5 \mathrm{D}$ upstream, $14.5 \mathrm{D}$ downstream of the cylinder, $6.5 D$ on either side of the cylinder, and $4 D$ in the spanwise direction. The Reynolds number, based on the uniform inlet flow velocity and the side length of the square cylinder, is 22,000. This flow configuration, both the geometry and the Reynolds number, is identical to the one used in the 1995's workshop and in [49]. Furthermore, the grid used is identical to the one 
employed by $\mathrm{Hu}$ [49]. It is a $160 \times 112 \times 16$ nonuniform, structured, Cartesian grid, becoming denser around the cylinder. The details of the grid structure can be found in [49].

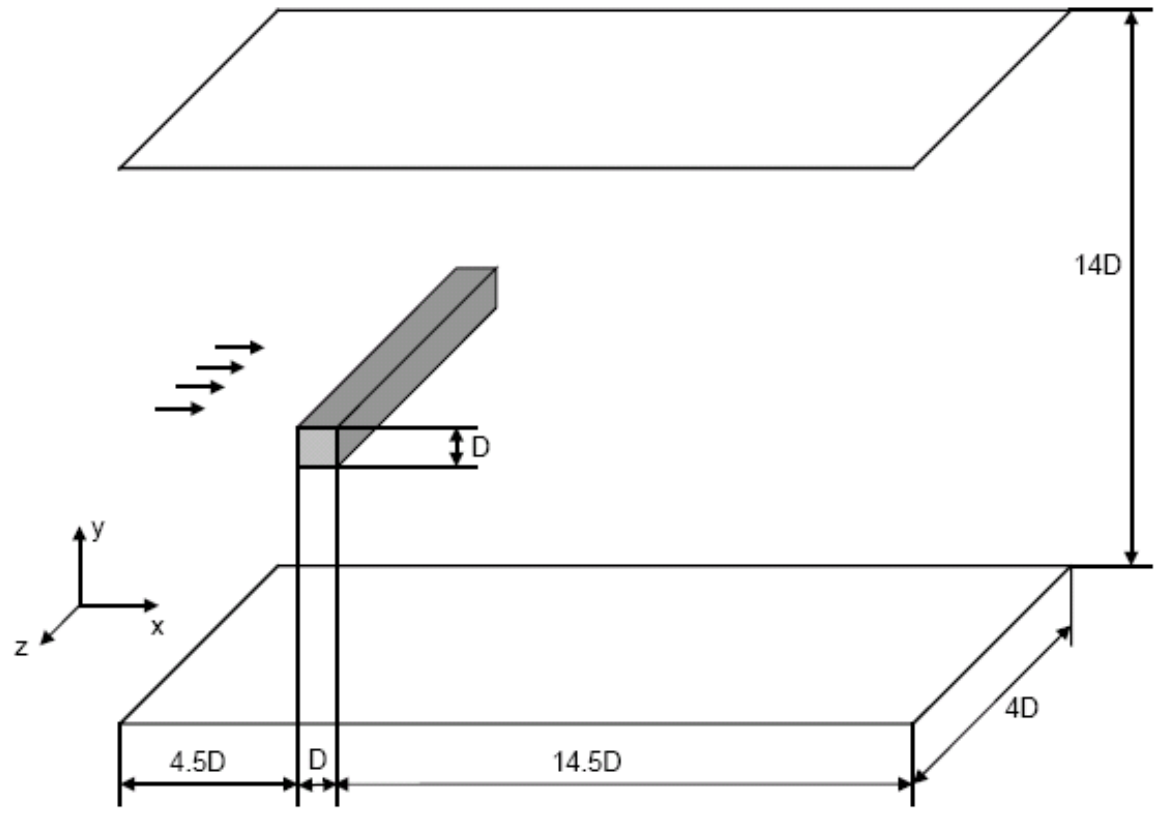

Figure 5.15: Schematic of flow past a square cylinder [49]

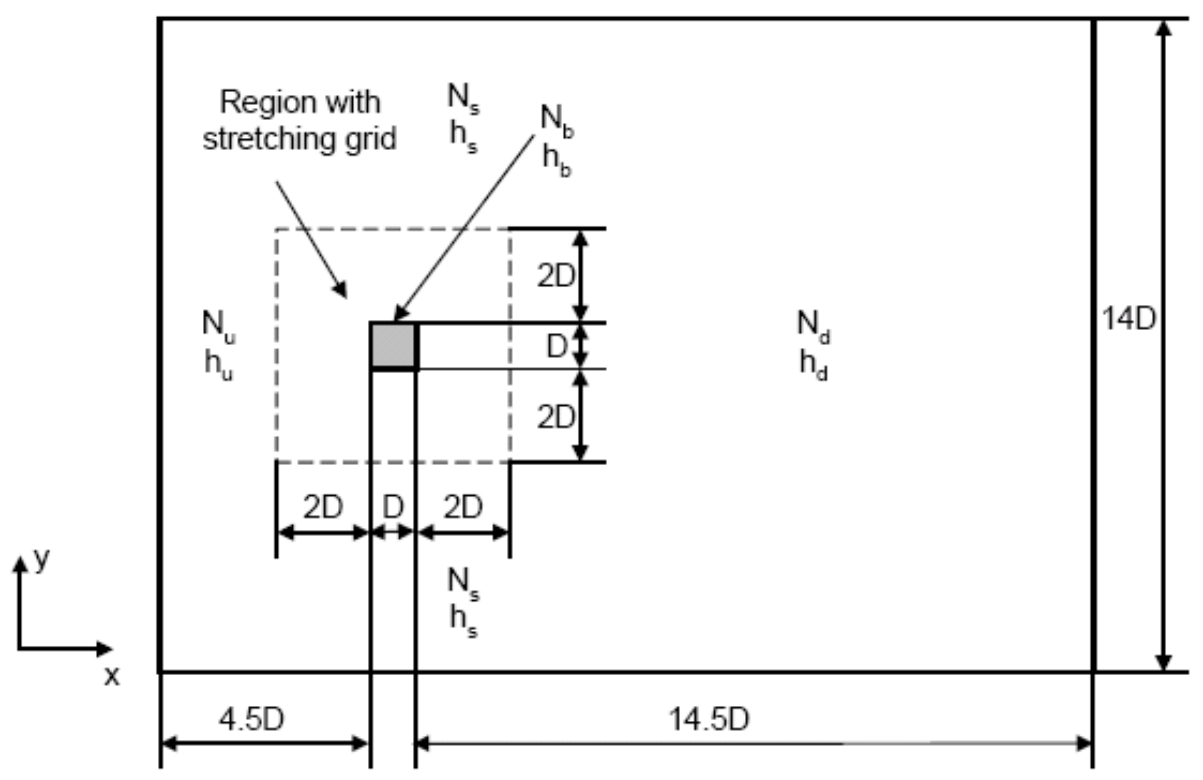

Figure 5.16: Grid distribution on the $x y$-plane for flow past a square cylinder [49] 
As to the boundary conditions, a uniform flow velocity is prescribed at the inlet. At the outlet an outlet boundary condition is used which ensures global mass conservation. The symmetry boundary conditions are applied in the normal direction at the upper and lower surfaces of the domain. In the spanwise direction the periodic boundary condition is applied. Also, the no-slip conditions are employed on the four cylinder surfaces. It is kept aware that the wall layer on the cylinder surfaces is not adequately resolved with the current grid resolution, albeit a stretched mesh is used. However, as a commonly accepted practice, an enforcement of the wall-condition is always better than doing nothing.

As for the SGS model, the dynamic eddy-viscosity model with the box filter for both grid filtering and the test filtering is used. The definition of the filter widths, as well as their justification, are given in [49]. A fourth order central differencing is applied to the convection terms, combined with a switching to QUICK (due to numerical stability) once the grid Peclet number exceeds 2. The computations were run parallel on 4 processors with varying grid densities. The time marching calculation is started with the fluid at rest. A constant time step of $2.2 \times 10^{-3}$, non-dimensionalized by $D / U_{0}$, is used. The calculation is considered complete when a fully developed state is reached, in which the turbulence quantities do not change in the mean. Results are then obtained by analyzing the flow data in the fully developed state.

Figure 5.17 display contours of the instantaneous and long-time averaged streamwise velocities. As can be seen from the instantaneous velocity field, the wake flow is unsteady. Indeed vortex shedding occurs. The average field, as expected exhibits a symmetric distribution.

The variation of the mean (long-time averaged) streamwise velocity along the centerline of the square cylinder is compared to those of Lyn et al. [62] and $\mathrm{Hu}[49]$ in Figure 5.18a. As can be seen from the figure the agreement of the present result with the others in the downstream region is in favorable agreement. However, it should be kept in mind that the numerical analyses performed by 


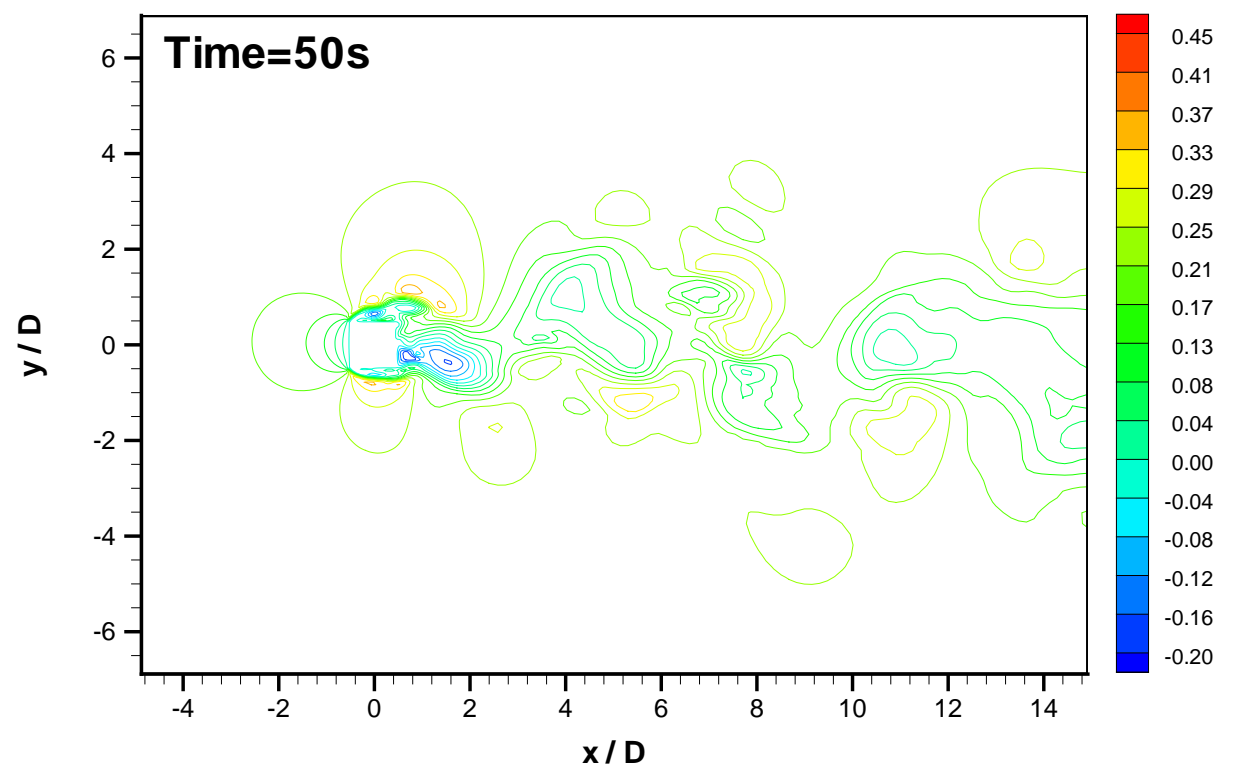

a)

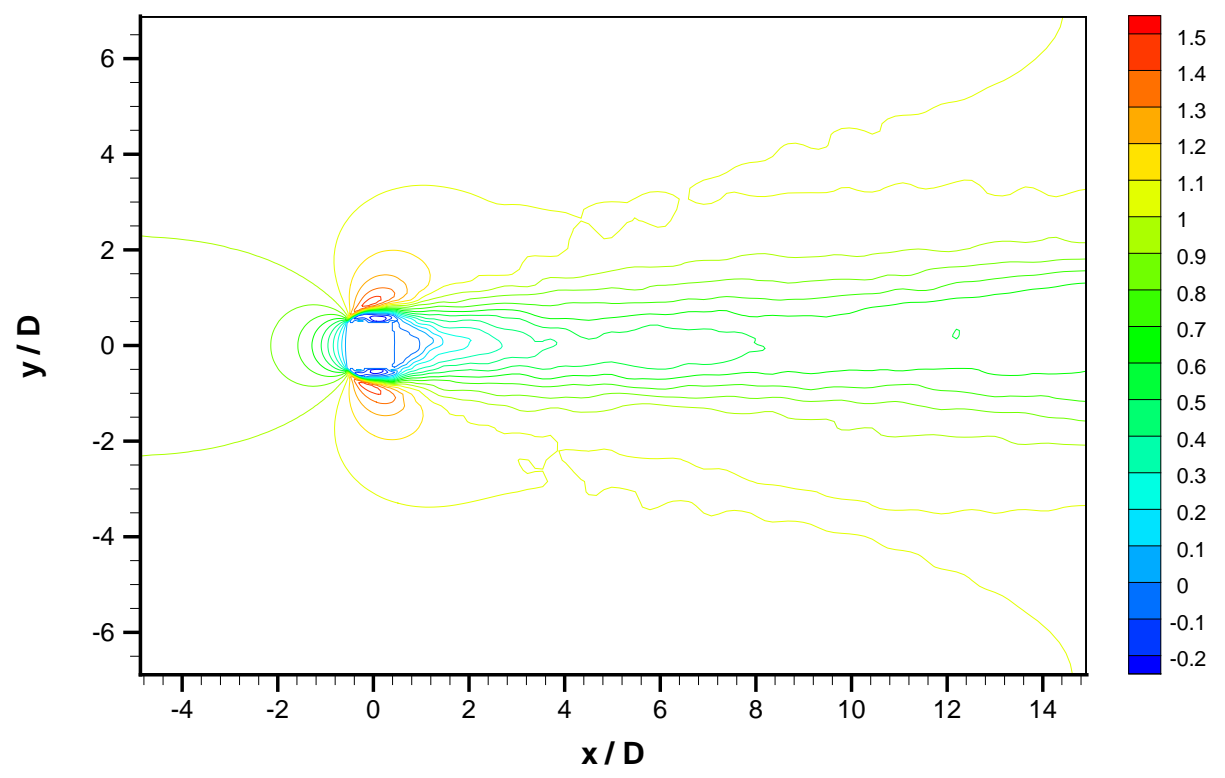

b)

Figure 5.17: Contours of streamwise velocity in the center $x y$-plane a) instantaneous, b) mean

different researchers also exhibit great variance (See Figure 5.18b). Comparison of Figures 5.18a and 5.18b indicates that the present result is within the scatter of the other numerical results. 


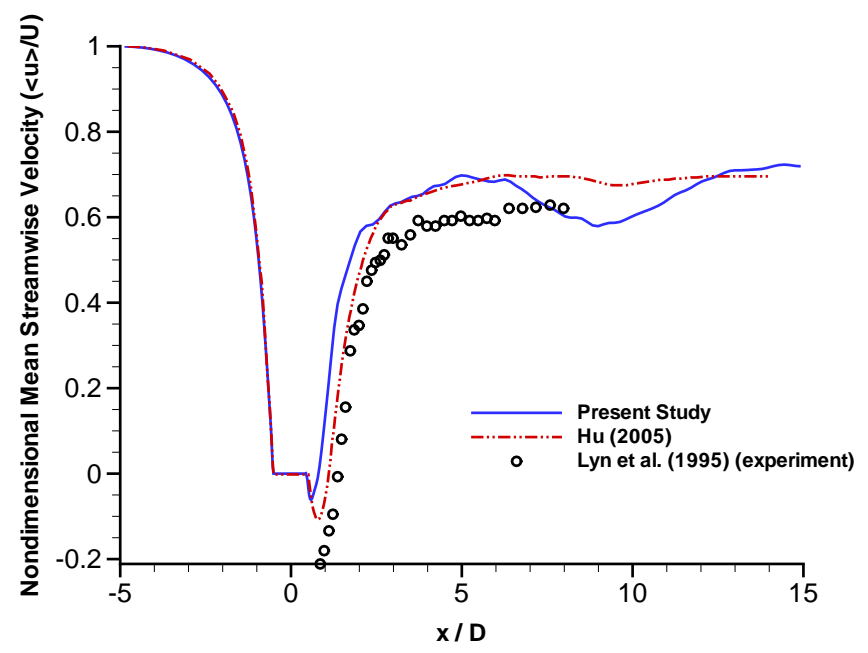

a)

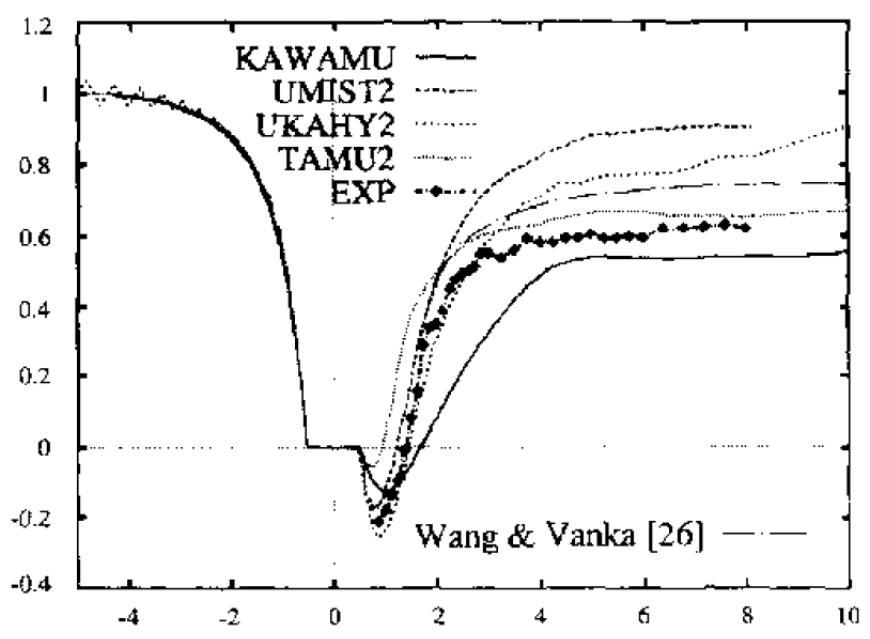

b)

Figure 5.18: Mean streamwise velocity along the centerline of square cylinder a) Present study, b) results from Rottach-Egern workshop (Reprinted from [63] with permission)

Figure 5.19 displays the time history of streamwise velocity at $(1 D, 0.5 D)$ and the corresponding PSD diagram calculated using Fast Fourier Transform (FFT). The figure brings out a dominant peak at the shedding frequency of $0.3 \mathrm{~Hz}$. Based on the cylinder diameter, $D$, and the inlet velocity, $U_{0}$, this frequency corresponds to Strouhal number of 0.136 which is very close to the experimental value (0.132) reported by Lyn [62]. On the whole, detailed, quantitative comparisons with previously validated sequantial code predictions and the experiments, have verified the accuracy of the parallel DREAM code. 


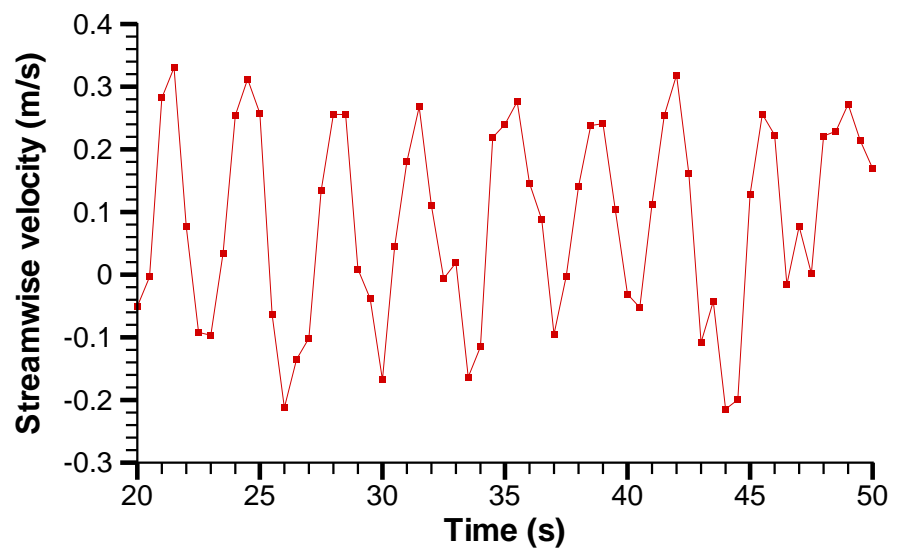

a)

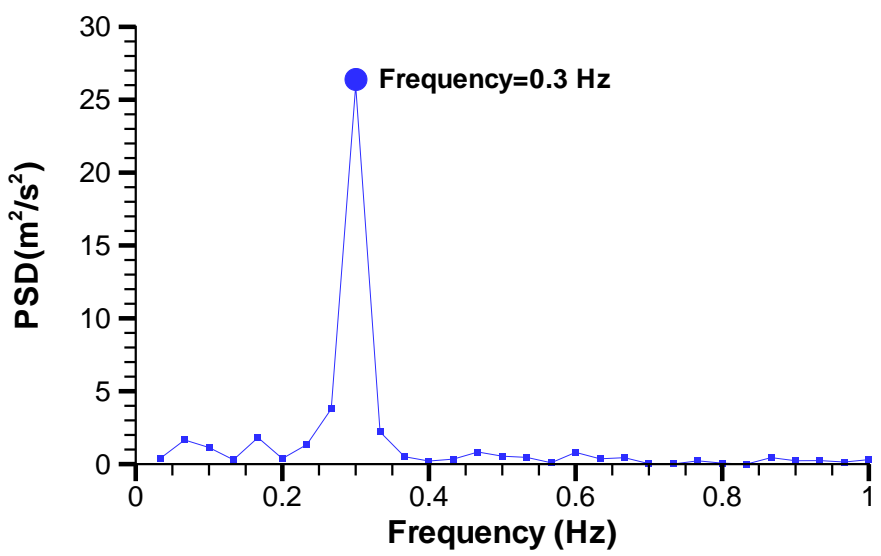

b)

Figure 5.19: Time history of mean streamwise velocity at (1D, 0.5D) and corresponding PSD spectrum 


\section{Chapter 6}

\section{Implementation of Quasi-Second Order Upwind Scheme into the DREAM}

This section presents implementation of the Quasi Second Order Upwind (QSOU) convection scheme [68] into the DREAM to improve its spatial accuracy for scalar transport as part of the overall goal on modeling contaminant (passive scalar) transport around a worker standing in front of an enclosing hood located in a ventilation chamber. Before the details of implementation are given, a brief background information on the behavior of spatial discreatization schemes is also presented. This section will end with verification study to determine the accuracy of the QSOU scheme.

\subsection{Introduction to QSOU Scheme}

The difficulty in CFD arises when trying to accurately solve for the convection of some scalar quantity, i.e. concentration, volume fraction, etc., as the accuracy is highly coupled with the numerical scheme as mentioned in Section 1.3. For accuracy, a second order scheme is generally required, as this order tends to limit the amount of numerical diffusion present in the calculations. However, for some cases second order schemes are known to produce wiggles which lead to negative values of the scalar. It is important to note that the negative scalar values must be eliminated and prevented by ensuring that a computational cell can not flux more scalar than it has, and it can not accept more scalar than than it has space 
for. Therefore, proper steps need to be taken. First order schemes, and certain hybrid schemes, are mostly used to prevent wiggles introduced through using central differencing schemes when the local cell Peclet number, $P e$, is larger than a certain value. However, these schemes do in fact introduce dissipation of the numerical solution, which may in fact exceed physical limits. A drawback to using a second order scheme arises in the computational cost, as the first order schemes run faster. A flux limited monotonic scheme called Quasi Second Order Upwind (QSOU) scheme is a promising approach in that it eliminates both the wiggles from the solution and the need to using a larger number of computational cells.

The QSOU scheme is a numerical method to solve the convection of a quantity based on the local flow direction, which then utilizes either a forward or backward differencing scheme to discretize the convection terms in the NavierStokes equation. Generally speaking, the QSUO scheme utilizes the minimum gradient at three cell locations, then adds this term to the convection based on the flow direction. In QSUO scheme the interpolated values are calculated in such a way that the convection scheme is strongly monotone. It selects an amount of upwind differencing that maximizes accuracy while maintaining strong-monotonicity.

The interpolated scalar density for fluxing a cell-centered quantity (concentration, generic scalar, etc.) is obtained in two steps. First, the derivative of scalar $\phi$ with respect to the distance in the logical coordinate direction $\left(\left.\frac{\partial \phi}{\partial s}\right|_{i}\right)$ in which fluxing is occurring is calculated from

$$
\begin{gathered}
\left.\frac{\partial \phi}{\partial x}\right|_{i j k}= \begin{cases}\operatorname{sign}\left(\Delta \phi_{i}\right) \min \left(\frac{\left|\Delta \phi_{i}\right|}{\left|\Delta \mathbf{X}_{i}\right|}, \frac{\left|\Delta \phi_{i-1}\right|}{\left|\Delta \mathbf{X}_{i-1}\right|}\right) & \text { if } \Delta \phi_{i} \Delta \phi_{i-1}>0 \\
0 & \text { if } \Delta \phi_{i} \Delta \phi_{i-1}<0\end{cases} \\
\Delta \phi_{i}=\phi_{i+1, j, k}-\phi_{i, j, k}
\end{gathered}
$$


and

$$
\Delta \mathbf{x}_{i}=\mathbf{x}_{i+1, j, k}-\mathbf{x}_{i, j, k}
$$

where $\mathbf{x}_{i j k}$ are the cell-center locations.

The QSOU scheme selects the gradient of minimum magnitude from the two gradients on either side of cell $(i, j, k)$, if these gradients have the same sign. Otherwise $\left.\frac{\partial \phi}{\partial x}\right|_{i j k}$ is set to zero. Figures 6.1 and 6.2 present the visual explanation of this approach on one-dimension. As can be seen from Figure 6.1, in order to obtain the gradient for the cell $i$, first the gradients shown in Figure 6.1 are calculated using the old time values of the scalar quantity. Then the minimum gradient of each pair [such as $(i-1, i)]$ shown in red, green and blue colors is selected and assigned to the cells; $i-1, i, i+1$ as shown in Figure 6.2.

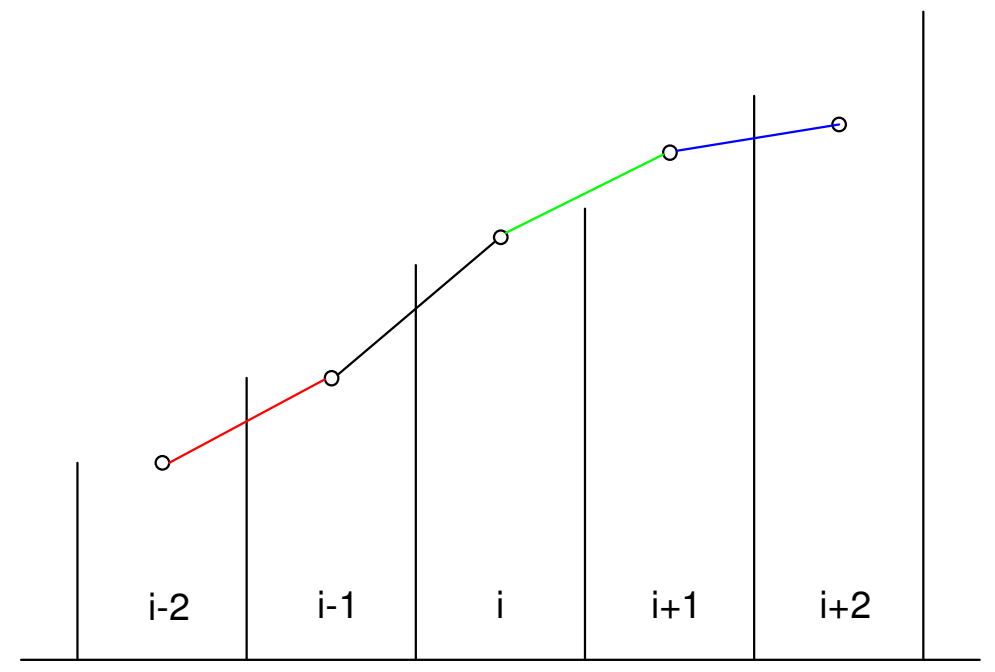

Figure 6.1: Variation of scalar quantity between adjacent cells

In the second step of the QSOU scheme calculations, the scalar quantity at the cell boundary is obtained by linear interpolation

$$
\phi_{a}= \begin{cases}\phi_{i, j, k}+\frac{\partial \phi}{\partial s}\left|\mathbf{x}_{a}-\mathbf{x}_{i, j, k}\right| & \text { if Convection }>0 \\ \phi_{i+1, j, k}-\frac{\partial \phi}{\partial s}\left|\mathbf{x}_{a}-\mathbf{x}_{i+1, j, k}\right| & \text { if Convection }<0\end{cases}
$$




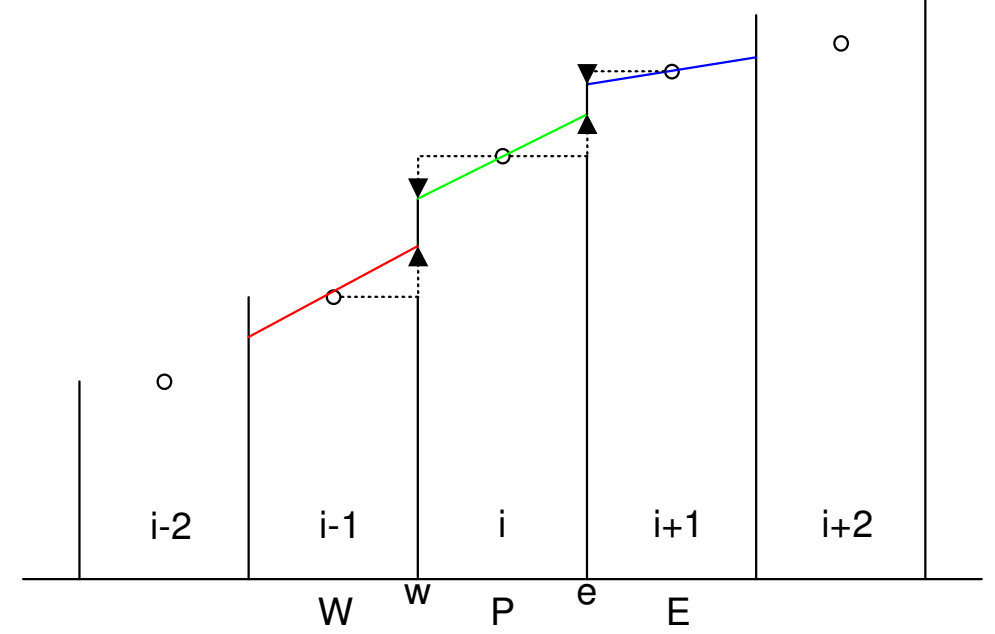

Figure 6.2: Variation of scalar quantity within cells

where $\mathbf{x}_{a}$ is the location of the cell face and $\phi_{a}$ is the magnitude of the scalar at that face. Special prescription are needed when cells are located next to computational boundaries. If face $a$ lies on a wall or and outflow boundary and cell $(i, j, k)$ is the fluid cell, one of whose faces is $a$, then

$$
\left.\frac{\partial \phi}{\partial s}\right|_{i, j, k}=0
$$

where the derivative is with respect to distance in the coordinate direction going into the wall. The derivatives in the other coordinate directions are unaffected, unless other faces of cell $(i, j, k)$ lie on computational boundaries. If face $a$ lies on an inflow boundary, then Equation 6.1 is used with modification to calculate the derivative with respect to the coordinate direction going into the inflow boundary. The modification is that the quantity and location of the cell center on the other side of the inflow boundary are taken to be the prescribed inflow quantity and center $x_{a}$ of cell face $a$, respectively. If face $a$ lies on an inflow or outflow boundary, Equation 6.4 is replaced with pure donor cell differencing [68]. 
The QSOU scheme is second-order accurate in space only when $\left(\phi_{i+1}-\right.$ $\left.\phi_{i}\right) / \Delta x$ is a constant independent of $i$, but the scheme will be nearly second-order accurate when $\left(\phi_{i+1}-\phi_{i}\right) / \Delta x$ varies slowly on the scale of the mesh spacing. When $\left(\phi_{i+1}-\phi_{i}\right) / \Delta x$ is constant, the scheme reduces to interpolated donor cell differencing [68].

\subsection{Formulation and Implementation}

The general, three-dimensional scalar transport equation is given as

$$
\frac{\partial}{\partial t}(\rho \phi)+\frac{\partial}{\partial x_{j}}\left(\rho u_{j} \phi\right)=\frac{\partial}{\partial x_{j}}\left(\Gamma \frac{\partial \phi}{\partial x_{j}}\right)
$$

where $\Gamma$ represents the diffusion coefficient, and it is zero in only pure convection cases which are used to verify this method in Sections 6.3.1 and 6.3.2. Since the QSOU scheme is only applied to the convection terms, the diffusion component of Equation 6.6 may be dropped for derivation purposes only. Thus the discretization of the convection term in three-dimensions is developed through integration of Equation 6.6) which gives

$$
\frac{1}{\Delta t}\left[\left(\rho \phi_{P}\right)-\left(\rho \phi_{P}\right)^{0}\right] \Delta V-\left\{\begin{array}{l}
{\left[A_{e}(\rho u \phi)_{e}-A_{w}(\rho u \phi)_{w}\right]+} \\
{\left[A_{n}(\rho u \phi)_{n}-A_{s}(\rho u \phi)_{s}\right]+} \\
{\left[A_{t}(\rho u \phi)_{t}-A_{b}(\rho u \phi)_{b}\right]}
\end{array}\right\} \approx 0
$$

where the superscript 0 represents the old time level and the subscript $P$ represents cell centers. All other subscripts, given by lower-case letters, denote cell faces. $\Delta V$ and $A$ represent cell volume and area of a cell face, respectively. For the sake of simplicity, instead of the three-dimensional equation (Equation 6.6), the formulation will only be presented with respect to the convection term (Conv) in the $x$-direction. However, the method is general and can be easily extended to three-dimensions. When the convection term is 
on the left hand side of Equation 6.6, it can be written as

$$
\operatorname{Conv}=A_{e}(\rho u \phi)_{e}-A_{w}(\rho u \phi)_{w}
$$

Letting

$$
F=\rho u A
$$

one can write the convection term as follows

$$
\text { Conv }=F_{e} \phi_{e}-F_{w} \phi_{w}
$$

According to the QSOU scheme, the face quantities $\left(\phi_{e}\right.$ and $\left.\phi_{w}\right)$ are calculated from cell center values as:

$$
\begin{gathered}
\phi_{e}= \begin{cases}\phi_{P}+\left.\frac{\partial \phi}{\partial x}\right|_{P}\left(x_{a}-x_{i}\right) & \text { if } F_{e}>0 \\
\phi_{E}-\left.\frac{\partial \phi}{\partial x}\right|_{E}\left(x_{i+1}-x_{a}\right) & \text { if } F_{e}<0\end{cases} \\
\phi_{w}= \begin{cases}\phi_{W}+\left.\frac{\partial \phi}{\partial x}\right|_{W}\left(x_{i-1}-x_{a}\right) & \text { if } F_{w}>0 \\
\phi_{P}-\left.\frac{\partial \phi}{\partial x}\right|_{P}\left(x_{a}-x_{i}\right) & \text { if } F_{w}<0\end{cases}
\end{gathered}
$$

Substituting expressions for quantities at the cell face into Conv (Equation 6.10), one can write the convection terms in a more compact way as given below

$$
\begin{aligned}
F_{e} \phi_{e} & =\left[\phi_{P}+\left.\frac{\partial \phi}{\partial x}\right|_{P}\left(x_{a}-x_{i}\right)\right]\left[\left|F_{e}, 0\right|\right] \\
- & {\left[\phi_{E}+\left.\frac{\partial \phi}{\partial x}\right|_{E}\left(x_{i+1}-x_{a}\right)\right]\left[\left|-F_{e}, 0\right|\right] } \\
F_{w} \phi_{w} & =\left[\phi_{W}+\left.\frac{\partial \phi}{\partial x}\right|_{W}\left(x_{i-1}-x_{a}\right)\right]\left[\left|F_{w}, 0\right|\right] \\
& -\left[\phi_{P}+\left.\frac{\partial \phi}{\partial x}\right|_{P}\left(x_{i}-x_{a}\right)\right]\left[\left|-F_{w}, 0\right|\right]
\end{aligned}
$$


where the sign [| |] means maximum value of its contents. Moving the convection terms to the right hand side and writing more explicitly yield

$$
\text { Conv }=\left\{\begin{array}{cc}
\phi_{W}\left[\left|F_{w}, 0\right|\right]-\phi_{P}\left[\left|-F_{w}, 0\right|\right] & -\phi_{P}\left[\left|F_{e}, 0\right|\right]+\phi_{E}\left[\left|-F_{e}, 0\right|\right] \\
+\left.\frac{\partial \phi}{\partial x}\right|_{W}\left(x_{i-1}-x_{a}\right)\left[\left|F_{w}, 0\right|\right] & +\left.\frac{\partial \phi}{\partial x}\right|_{P}\left(x_{i}-x_{a}\right)\left[\left|-F_{w}, 0\right|\right] \\
-\left.\frac{\partial \phi}{\partial x}\right|_{P}\left(x_{a}-x_{i}\right)\left[\left|F_{e}, 0\right|\right] & -\left.\frac{\partial \phi}{\partial x}\right|_{E}\left(x_{i+1}-x_{a}\right)\left[\left|-F_{e}, 0\right|\right]
\end{array}\right\}
$$

The first four terms of (Equation 6.15) are identical to the first order upwind scheme whereas the last four terms (gradients), resulting from QSOU scheme are additional terms and are evaluated explicitly from the old time values. Therefore, these terms are not added to the coefficients matrix, instead they are substituted into constant part of the source term, $S_{c}$. As mentioned before, the coefficients remain unchanged as in first order upwind scheme:

$$
\begin{gathered}
a_{E}=D_{e}+\left[\left|-F_{e}, 0\right|\right] \\
a_{W}=D_{w}+\left[\left|F_{w}, 0\right|\right] \\
a_{P}=D_{w}+D_{e}+D_{s}+D_{n}+D_{b}+D_{t} \\
+\left[\left|F_{e}, 0\right|\right]+\left[\left|-F_{w}, 0\right|\right]+\left[\left|F_{n}, 0\right|\right]+\left[\left|-F_{s}, 0\right|\right]+\left[\left|F_{t}, 0\right|\right]+\left[\left|-F_{b}, 0\right|\right]
\end{gathered}
$$

with a modified source term given as

$$
\begin{aligned}
S_{c}=\rho \Delta V \frac{\phi^{0}}{\delta t}+ & \left.\frac{\partial \phi}{\partial x}\right|_{W}\left(x_{i-1}-x_{a}\right)\left[\left|F_{w}, 0\right|\right]+\left.\frac{\partial \phi}{\partial x}\right|_{P}\left(x_{i}-x_{a}\right)\left[\left|-F_{w}, 0\right|\right] \\
& -\left.\frac{\partial \phi}{\partial x}\right|_{P}\left(x_{a}-x_{i}\right)\left[\left|F_{e}, 0\right|\right]-\left.\frac{\partial \phi}{\partial x}\right|_{E}\left(x_{i+1}-x_{a}\right)\left[\left|-F_{e}, 0\right|\right]
\end{aligned}
$$




\subsection{Verification of the Scalar Solver}

\subsubsection{Test Case I: Diagonal Flow}

The accuracy of the code was assessed by applying it to a test case given in the KIVA manual [68]. In this test case, a scalar signal which is initially unity on a square that has five cells to a side and is zero, otherwise is convected through a two-dimensional grid of square cells with a uniform velocity directed at $45^{\circ}$ angle to the grid directions $(u=1, v=1)$ (See Figures 6.3a and 6.4a). The CFL number used is 0.2 with a $20 \times 20 \times 1$ grid and a time step of $\Delta t=0.01$. The scalar field is initially unity (See Figures 6.3 and 6.4 ) on a square that has five cells to a side and is zero otherwise. Since the physical diffusivity of the generic scalar is set to zero, the only source for diffusion mechanism remains the numerical diffusion. For both the KIVA [68] and DREAM codes, the initial conditions and the predicted scalar fields at the end of 25 time steps are given in Figures 6.3 and 6.4, respectively. It is evident from the comparison of Figures $6.3 \mathrm{~b}$ and $6.4 \mathrm{~b}$ that the prediction of DREAM is in very good agreement with that of the KIVA [68] in terms of the final distribution, and the maximum and minimum values of the scalar.

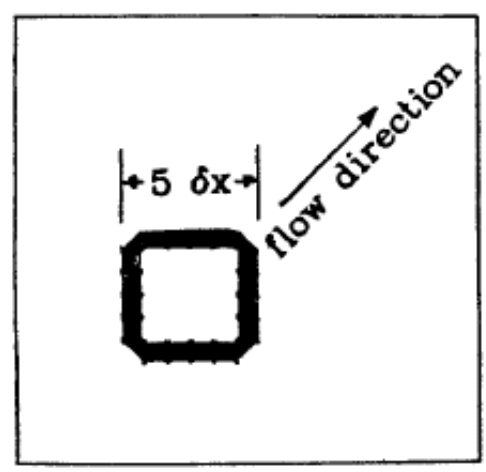

a)

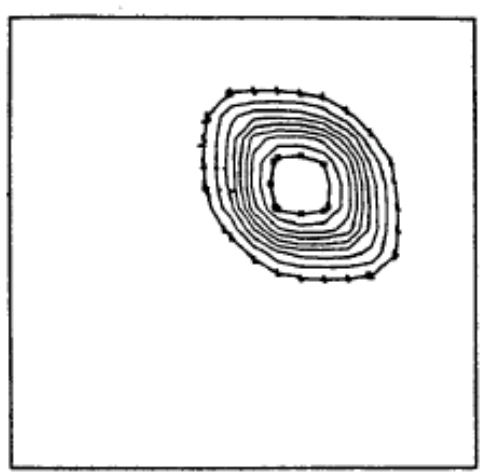

b)

Figure 6.3: Scalar fields for KIVA [68] a) Initial signal, b) Signal after 25 time steps 


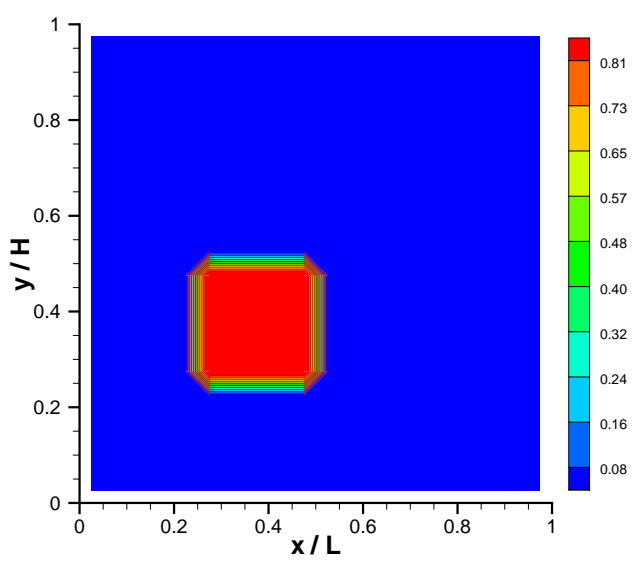

a)

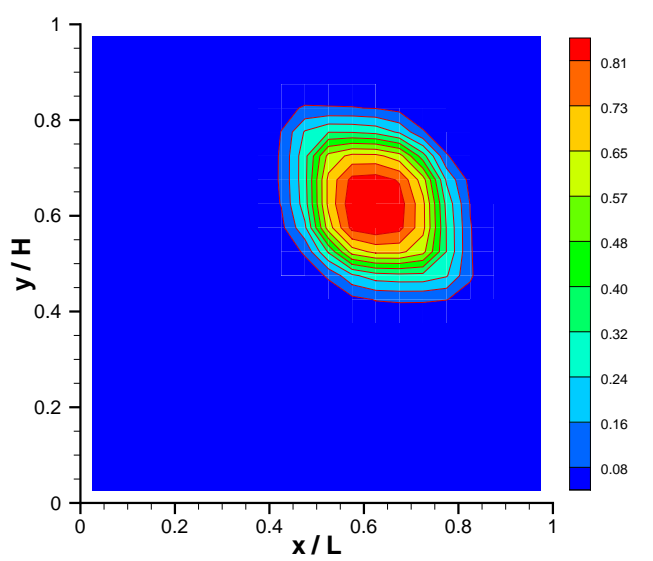

b)

Figure 6.4: Scalar fields for DREAM a) Initial signal, b) Signal after 25 time steps

\subsubsection{Test Case II: Rotational Flow}

As the result shown thus far in comparison of the DREAM simulation of scalar transport quantity in a diagonal flow field to those reported in the KIVA manual [68], the DREAM simulation compare very favorable to those of the KIVA [68]. Thus, focus shifts to a more interesting transport phenomena: Transient scalar transport in an imposed rotational (or vortex) flow field: In the specified square computational domain, each point moves around the center point with a constant angular velocity, $\omega$. The tangential velocity $V$ at each point can be simply calculated from $\omega r$, where $r$ is the distance from that point to the center (radius). As a result, every point provides a different $u$-velocity component and $v$-velocity component, which in Cartesian coordinate system should be a function of $x$ and $y$ position.

The computational domain considered here is a square with side length, $L$, equal to $1.0 \mathrm{~m}$. The origin is placed at bottom left corner of the square. A clockwise rotational flow field, given in Eqs. 6.20- 6.21, is imposed.

Angular velocity:

$$
\omega=2 r \exp (-r)^{2}
$$


Tangential velocity components:

$$
u=\omega \frac{y}{r} \quad v=-\omega \frac{x}{r}
$$

where $r$ is the radius extending from the center point of the domain to the point under consideration.

The scalar signal is initially unity on a square covering a region from $x=0.3 \mathrm{~m}$, $y=0.3 \mathrm{~m}$ to $x=0.4 \mathrm{~m}, y=0.4 \mathrm{~m}$ and is zero otherwise. The velocity and initial scalar fields are shown in Figure 6.5. Overall, the scalar signal inside the domain will be carried by the convective field and experiences a rotational motion around the center point. Since the diffusivity of the scalar was set to zero, while the signal is rotating, it is only subject to a purely numerical diffusion process, which weakens the strength of the signal and makes it finally flat.

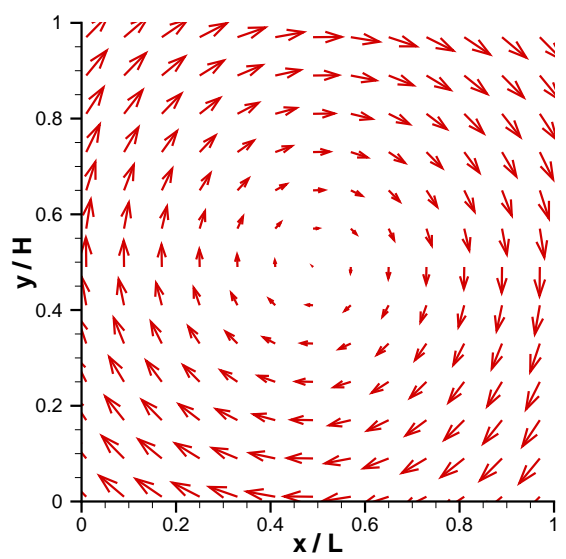

a)

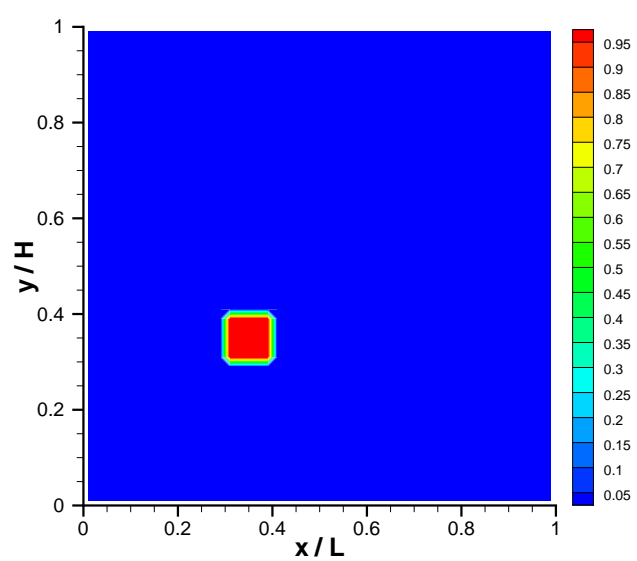

b)

Figure 6.5: Velocity field (a), and initial scalar field (b)

In the calculations, the viscosity and density of the fluid are taken as $4.673 \times 10^{-5} \mathrm{~kg} / \mathrm{m} \mathrm{s}$ and $1.0 \mathrm{~kg} / \mathrm{m}^{3}$, respectively. For comparative purposes the simulations are first carried out on a $50 \times 50 \times 1$ grid using first order upwind and QSOU schemes for spatial discretization of convective terms. With a time step of 0.01s simulations are run for 34 time steps at whose point the vortex made a full cycle. Symmetry boundary conditions are imposed for the scalar. 
Figures 6.6 and 6.7 show the results for this type of flow field, and as expected, the QSOU is less dispersive than first order upwind scheme. This also obvious from Table 6.1 where the maximum of values of the scalar are presented.

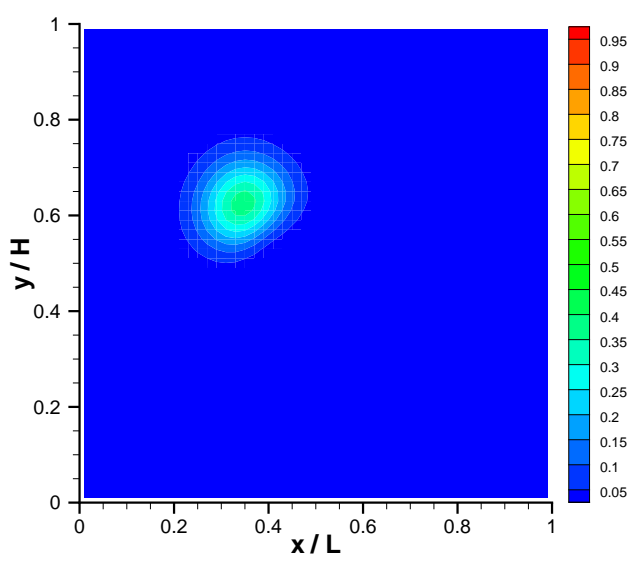

a)

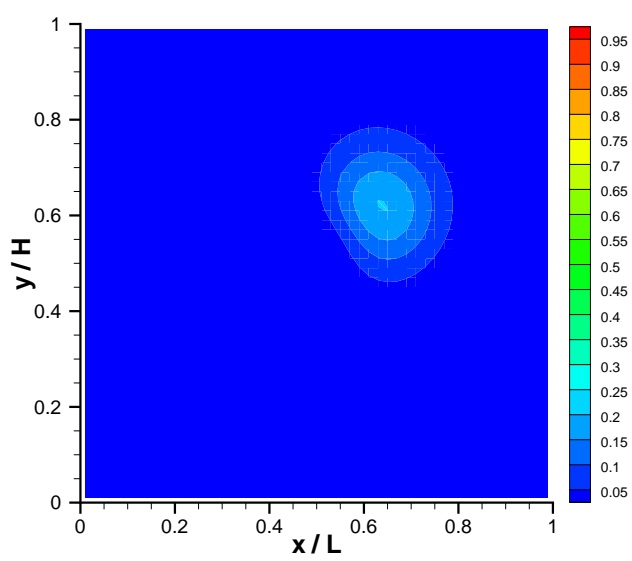

b)

Figure 6.6: Scalar field predicted using first order upwind scheme at the end of a) a quarter cycle, b) a half cycle

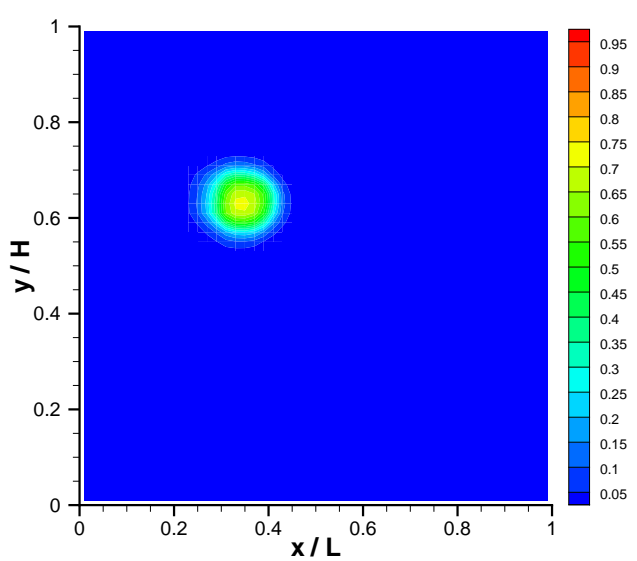

a)

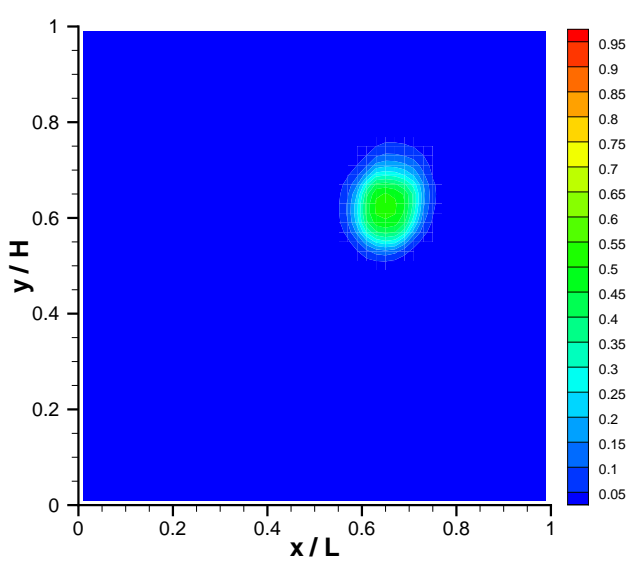

b)

Figure 6.7: Scalar field predicted using QSOU scheme at the end of a) a quarter cycle, b) a half cycle

As seen from the table, the improvement that QSOU brings is considerable. However, since the grid is very coarse, the predictions looks still very dispersive. Therefore, the dependency of the numerical diffusion on the grid should be investigated as well. To this end, the calculations are repeated on a finer mesh 
Table 6.1: Maximum values of the scalar

\begin{tabular}{lcc}
\hline Time & $1^{\text {st }}$ order upwind & QSOU \\
\hline End of quarter cycle $(0.8 \mathrm{sec})$ & 0.38 & 0.72 \\
End of half cycle $(1.7 \mathrm{sec})$ & 0.20 & 0.52 \\
\hline
\end{tabular}

composed of $200 \times 200 \times 1$ cells. Due to stability constraints the time step is reduced to 0.001 s correspondingly. Figure 6.8 illustrates the scalar field obtained on the fine grid. The improvement with higher mesh resolution is evident. Not only the initial shape (square) of the scalar signal is better preserved, but also the maximum value of the scalar at reported instants are higher. At the end of quarter cycle the maximum value is 0.997 whereas, it is drops only to 0.97 at the end of half cycle.
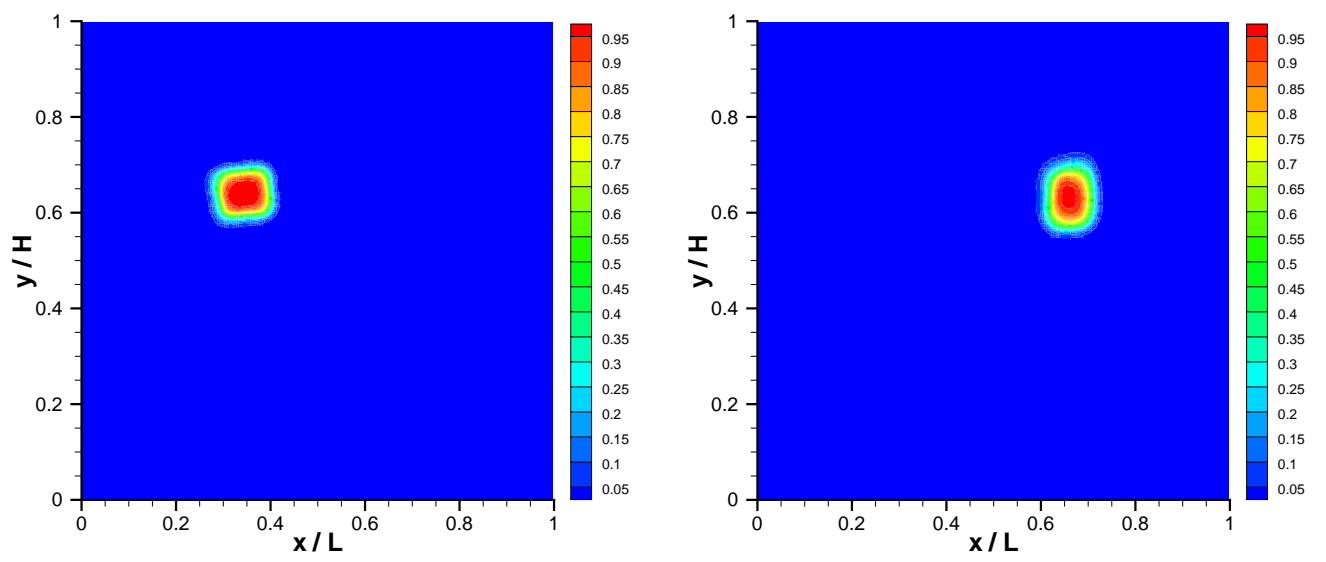

Figure 6.8: Passive scalar field on a fine mesh at the end of a) a quarter cycle $(\max =0.997), b)$ a half cycle $(\max =0.97)$

On the whole, based on the very good agreement with the solution reported in the KIVA manual in Test Case I, and outperformance the first order upwind scheme in Test Case II, it can be concluded that QSOU scheme was properly implemented into DREAM code and is ready to be used for more complex flows. 


\section{Chapter 7}

\section{Implementation of Immersed Boundary Method into the DREAM}

There are two approaches in the literature, namely, body-fitted grid approach and Immersed Boundary (IB) method, to solve flows around solid bodies. The former one, being the conventional approach, is based on solving discretized Navier-Stokes equations on a computational grid that conforms the solid body, and allows easy imposition of appropriate boundary conditions. However, this approach suffers from two main drawbacks, especially when the geometry of the immersed body has curvatures. First, it requires curvilinear or unstructured grids which means foregoing the advantages of numerical accuracy and computational efficiency associated with simple orthogonal grids. Second, generating high quality body-fitted structured grids for complex geometries can be extremely time consuming and cumbersome [69]. Hence, grid generation, which can be a very labor-intensive task even with robust grid generators, becomes an additional major step in CFD analyses. Furthermore, in modeling of moving boundary flows, transient re-gridding is required further increasing the computational overhead. Alternatively, these problems can be alleviated by the so-called Immersed Boundary (IB) method in which the surface geometry of the solid (immersed) body only needs to be mapped onto the underlying computational grid without any necessity of coinciding the grid points with the

points defining the IB. The key idea underlying the IB method is to model the IB 


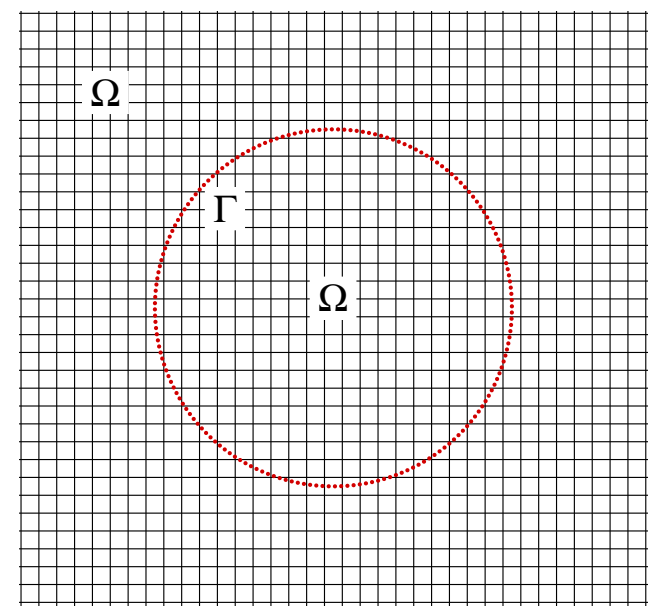

Figure 7.1: Flow domain $(\Omega)$ with a circular immersed boundary $(\Gamma)$

(solid body) by representing its shape through an external force field equivalent to the one that would be exerted by a physical solid body. More clearly, rather than imposing boundary conditions on the surface of the solid body, the correct force field is imposed to simulate the immersed solid boundary as the fluid passes over it. Thus, a flow around a solid body can be computed in a simple domain on a regular orthogonal grid by solving the governing flow equations with the external force term added to represent its effect [70].

The concept of IB was first introduced by Peskin $[71,72]$ in modeling of blood flow in the human heart which naturally possesses a flexible boundary. In order to account for the interaction between the fluid and the flexible solid boundary, Peskin used a mixed Euler-Lagrangian finite difference method and solved the governing equations on a Cartesian grid. The IB is represented by a set of massless elastic fibers whose location is tracked in a Lagrangian fashion by a collection of massless points that move with the local fluid velocity. Since these pioneering studies, numerous modifications and refinements have been made and the proposed methods have been applied to similar flows involving flexible boundaries [73-75]. 
The IB method has also been applied to tackle with flow problems involving solid rigid boundaries. Lai and Peskin [76] proposed a formally second-order accurate IB method to simulate flows past a rigid cylinder. In the above papers, both the flexible and rigid boundaries are simply mimicked by a stiff spring connecting the boundary points to their target positions and the boundary force is generated by deviation of markers from their target positions. This approach, indeed, is a particular case of feedback forcing formulation proposed independently by Goldstein et al. [70] to simulate a flow over solid boundaries within the spectral method framework. Their approach, known as Virtual Boundary Method, treats the solid boundary as a force generator where the force density is calculated by a feedback method formulated on the difference between the predicted velocity and the actual velocity of the boundary. Saiki and Biringen [77] used the same forcing scheme to compute the low-Reynolds number $(\mathrm{Re} \leq 400)$ flow over stationary, rotating and horizontally oscillating circular cylinders using finite difference formulation. Their numerical results compared very well with the experimental results proving that the feedback forcing IB method is capable of handling solid boundary problems, including moving boundaries. Later, Lee et al. [78] successfully applied the same approach for flows over different configurations of stationary and oscillating cylinders.

However, feedback forcing IB methods is known to have sometimes severe stability problems causing a restriction of CFL number. The reason of this problem is mainly the free constants imposed in the forcing term. Since these numbers have to be big numbers and the feedback force is calculated and distributed explicitly into the fluid at every discrete time step, the problem becomes very stiff which has consequence of the small time step (small CFL number) [79]. Accordingly, Goldstein had to keep CFL number below $O\left(10^{-2}\right)$ in order to avoid the spurious oscillation in simulating the start-up flow around a cylinder. However, as stated by Fadlun [80], despite this drawback, the method is always more advantageous than the classic body-fitted grid approach and it has been recently applied to the simulation of three-dimensional flows by Goldstein et 
al. [70], Goldstein and Tuan [81], Saiki and Biringen [77] and Arthurs et al. [82]. On the other hand, there are studies that partially modifies the momentum forcing to make the time step less restrictive. This modifications are based on not-so-straightforward implicit forcing which requires the re-evaluations of the coefficients related to the implicitly treated terms. By using an implicit formulation for the feedback momentum forcing, Le et al. [83] could reach to CFL number of 0.5 to 1.

Alternative to feedback forcing IB method, recently, Mohd-Yusof [84] proposed a direct forcing method within the spectral context, where forcing is directly computed from momentum equations and applied to a set of points adjacent to the surface and interior to the immersed body in order to reverse the tangential velocities such that the desired velocities can be achieved on the boundary. Conceptually, this is equivalent to direct imposition of the exact velocity on the solid boundary through an interpolation procedure. The major advantage brought by this approach is that since the force field is directly computed from the momentum equations, the computation does not suffer from time step restriction as it occurs in feedback forcing IB method. Fadlun et al. [80] further extended this approach to a finite difference formulation on a staggered grid system. Direct forcing IB methods have been used successfully to simulate flow around spherical particles in particle-gas two-phase flow [84], three-dimensional complex-flow problems [80, 84], and flow with moving object [85]. Other direct forcing IB methods incorporate different ways to compute forcing term [86, 90]. However, in the traditional direct forcing approach the determinations of the forcing locations and their magnitudes may not be straightforward on the Eularian grids, especially for time evolving moving boundary flows. Furthermore, resolution of IB depends on grid resolution. However, similar to feedback forcing approach, there are modifications to overcame these drawbacks $[79,83]$.

On the whole, in their original forms, both approaches have some drawbacks and various remedies have been proposed for treating these deficiencies in the 
literature. In the present study, the traditional feedback forcing IB method [77] has been chosen due to its adequate accuracy and relative simplicity. In the subsequent sections, the formulation of feedback momentum forcing and interpolation scheme followed by validation of DREAM code on a twodimensional test case are presented.

\subsection{Feedback Momentum Forcing}

The feedback forcing function proposed by Goldstein et al. [70] is expressed as

$$
\mathbf{F}\left(\mathbf{x}_{\mathbf{s}}, t\right)=\alpha \int_{0}^{t}\left[\mathbf{U}\left(\mathbf{x}_{\mathbf{s}}, t\right)-\mathbf{v}\left(\mathbf{x}_{\mathbf{s}}, t\right)\right] d t+\beta\left[\mathbf{U}\left(\mathbf{x}_{\mathbf{s}}, t\right)-\mathbf{v}\left(\mathbf{x}_{\mathbf{s}}, t\right)\right]
$$

where $\mathbf{U}\left(\mathbf{x}_{\mathbf{s}}, t\right)$ is the velocity at a discrete IB point $\left(\mathbf{x}_{\mathbf{s}}\right)$, and $\mathbf{F}\left(\mathbf{x}_{\mathbf{s}}, t\right)$ is the force acting exactly on that point. The $\mathbf{v}\left(\mathbf{x}_{\mathbf{s}}, t\right)$ is boundary velocity which can be used to define deformation, rotation and translation of bodies in space and time. Velocity difference, actually the current error; $\left[\mathbf{U}\left(\mathbf{x}_{\mathbf{s}}, t\right)-\mathbf{v}\left(\mathbf{x}_{\mathbf{s}}, t\right)\right]$ determines the amount of feedback momentum forcing that brings the fluid velocity, $\mathbf{U}\left(\mathbf{x}_{\mathbf{s}}, t\right)$ to the desired wall (boundary) velocity $\mathbf{v}\left(\mathbf{x}_{\mathbf{s}}, t\right)$. If the boundary is fixed, which is the case in the present study, $\mathbf{v}\left(\mathbf{x}_{\mathbf{s}}, t\right)$ becomes zero and Equation 7.1 simplifies to:

$$
\mathbf{F}\left(\mathbf{x}_{\mathbf{s}}, t\right)=\alpha \int_{0}^{t} \mathbf{U}\left(\mathbf{x}_{\mathbf{s}}, t\right) d t+\beta \mathbf{U}\left(\mathbf{x}_{\mathbf{s}}, t\right)
$$

This particular form is analogous to PI controller, where P and I stand for the proportional and the integral parts, respectively. The proportional term provides feedback based on the current error, and the integral term provides feedback based on the accumulation of the past error. One can easily notice that the presented mechanism is also analogous to simple harmonic oscillator problem; a damped spring-mass system. Here, the mass can be considered as a small volume of fluid near $\mathbf{x}_{\mathbf{s}}$. 
The gains of $\alpha$ and $\beta$, appearing in Equations 7.1 and 7.2 are big negative flow dependent constants with dimensions of $\left[1 / T^{2}\right]$ and $[1 / T]$, respectively. They are determined by observing the response of $\mathbf{U}\left(\mathbf{x}_{\mathbf{s}}, t\right)$ once $\mathbf{F}\left(\mathbf{x}_{\mathbf{s}}, t\right)$ is applied. More clearly, $\alpha$ produces the natural oscillation frequency of the response, while $\beta$ dampens the oscillation of the response. For unsteady flows, $\alpha$ should be tuned so that the proportional term produces a response with a natural frequency greater than the highest frequencies present in the flow ensuring that the force field, $\mathbf{F}\left(\mathbf{x}_{\mathbf{s}}, t\right)$, tracks and responds correctly to the dynamic flow field. The overall steady state of the flow field is not affected by the oscillatory nature of boundary. However, for each combination of $\alpha$ and $\beta$ a different forcing function is added to the right hand side of the momentum equations, therefore a set of similar but slightly different flow fields are obtained for each of the solutions [77]. To examine the response of the immersed boundary to different $\alpha / \beta$ combinations, Saiki and Biringen [77] modeled a flow around a stationary cylinder at $R e=25$. Their findings suggest that higher values of the coefficients allow the method to respond faster to any unsteadiness in the flow field and act more efficiently in reinforcing the no-slip conditions. However, the method by which the time integral is evaluated will determine the maximum magnitude of $\alpha$. In this study the time integral is approximated simply as a Riemann sum:

$$
\int_{0}^{t} \mathbf{U}\left(\mathbf{x}_{\mathbf{s}}, t\right) d t \approx \sum_{j=1}^{N} \mathbf{U}\left(\mathbf{x}_{\mathbf{s}}, j\right) \Delta t
$$

Hence, Equation 7.2 is approximated by

$$
\mathbf{F}\left(\mathbf{x}_{\mathbf{s}}, t\right)=\alpha \sum_{j=1}^{N} \mathbf{U}\left(\mathbf{x}_{\mathbf{s}}, j\right) \Delta t+\beta \mathbf{U}\left(\mathbf{x}_{\mathbf{s}}, t\right)
$$

In Equations. 7.3 and 7.4, $N$ is the number of time steps $(\Delta t)$ advanced until a given time $t$. This integration scheme, for large gain $(\alpha)$, is clearly unstable for the simple harmonic oscillator problem. In practice, Goldstein et al. [70] reported that the solution to Navier-Stokes equations is still unstable for large 
enough $\alpha$ or $\beta$ but is stable for moderate values and insensitive to their exact values. In particular, for a second-order accurate Adams-Bashforth scheme, which is used in the present study, Goldstein et al. [70] found that the stability limit for the time step is approximately:

$$
\Delta t<\frac{-\beta-\sqrt{\left(\beta^{2}-2 \alpha k\right)}}{\alpha}
$$

$k$ being a problem dependent constant of order one.

One should note that the force field $\mathbf{F}\left(\mathbf{x}_{\mathbf{s}}, t\right)$ in Equation 7.4 is calculated at the boundary interface, i.e. at discrete IB points $\left(\mathbf{x}_{\mathbf{s}}\right)$, using the velocities exactly at the same locations. However, since the IB is generally defined in a manner independent of the computational grid, interpolation of the calculated velocities to the discrete IB points from the nearby computational grid points is required. Using the interpolated velocities the force field $\mathbf{F}\left(\mathbf{x}_{\mathbf{s}}, t\right)$ at IB is calculated from Equation 7.4 and then, using Equation 7.6, its effect is extrapolated (redistributed) back to computational grid points near the IB again. Figure 7.2 depicts this operation schematically. Sum of nearby forcing multiplied by weighting factor $\left(D_{i, j, k}\right)$ is the momentum forcing, $\mathbf{F}\left(\mathbf{x}_{\mathbf{s}}, t\right)$ of nearby grid points. The weighting factor is the same as the one used to interpolate fluid velocity to IB points and is calculated from the interpolation scheme described in the next section.

$$
\mathbf{F}_{i, j, k}=\frac{1}{N_{b}} \sum_{n=1}^{N_{b}} D_{i, j, k}\left(\mathbf{x}_{\mathbf{s}}\right) \mathbf{F}_{n}\left(\mathbf{x}_{\mathbf{s}}\right)
$$

In Equation 7.6, $N_{b}$ is the total number of IB points that have an impact on the computational grid point $(i, j, k)$. The method prescribed above is also known as 'area-weighted' method proposed by Saiki and Biringen [77].

In the spirit of IB method, the number of boundary points, $N_{b}$, can be independently increased for a sufficient resolution of IB without being restricted 
to the resolution of the computational grid. However, one should keep in mind that the accuracy of the solution can reach a limit by adding boundary points without increasing the computational grid resolution. A detailed study of the IB resolution requirement has been provided by Zhang and Zheng [91]. To prevent information 'leak' on the IB, the number of IB points should be at least two times the number of grid cells intercepted [92].

\subsection{Interpolation Scheme}

There are many interpolation methods with varying order of accuracy and computational cost. For a flow case involving moving rigid solid body, the interpolation weights need to be computed at every time step, which has a consequence of excessive computational burden just for interpolation. Therefore, one should select an interpolation scheme based on the accuracy level needed.

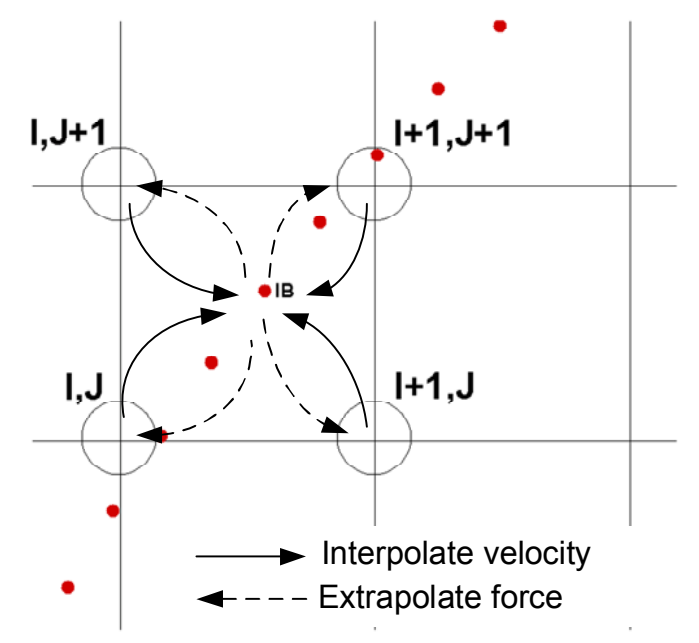

Figure 7.2: Schematic of interpolation/extrapolation operation for a twodimensional case. (IB): immersed boundary point, (I,J) pairs: computational grid point

In a pioneering work [72], Peskin used a first order cosine function for interpolation of velocities and extrapolation of calculated forces. More recently, Beyer [93] developed a second-order accurate representation of the IB in applications of Peskin's method. Goldstein et al. [70] interpolated velocities of grid points from IB points with a highly accurate spectral interpolation 
and extrapolated forcing term of immersed boundary points from grid points with linear interpolation. Saiki and Biringen [77] incorporated a bi-linear interpolation that is also first order accurate. This method of distributing the boundary forces results in an effective boundary thickness on the order of one grid cell, i.e. $O(\Delta x, \Delta y, \Delta z)$. The low order accuracy of bi-linear interpolation influences mainly the flow field in the immediate vicinity of the solid body; however, large scale features, which are the main interest of this study, are successfully captured by this method [77]. Therefore, in this study, bi-linear interpolation scheme is used to find surface velocities and to redistribute the effect of the forcing term back to the underlying grid points.

The bi-linear interpolation scheme in three-dimensions reads

$$
\mathbf{U}\left(\mathbf{x}_{\mathbf{s}}\right)=\sum_{i, j, k}^{i+1, j+1, k+1} D_{i, j, k}\left(\mathbf{x}_{\mathbf{s}}\right) \mathbf{U}_{i, j, k}
$$

where the weight $D_{i, j, k}\left(\mathbf{x}_{\mathbf{s}}\right)$ is defined as

$$
D_{i, j, k}\left(\mathbf{x}_{\mathbf{s}}\right)=d\left(x_{s}-x_{i}\right) d\left(y_{s}-y_{j}\right) d\left(z_{s}-z_{k}\right)
$$

In Equation 7.8

$$
\begin{aligned}
& d\left(x_{s}-x_{i}\right)=\frac{\left(x_{s}-x_{i}\right)}{\left(x_{i+1}-x_{i}\right)} \quad \text { if } \quad x_{i}<x_{s} \\
& d\left(x_{s}-x_{i}\right)=\frac{\left(x_{s}-x_{i}\right)}{\left(x_{i}-x_{i-1}\right)} \quad \text { if } \quad x_{i}>x_{s}
\end{aligned}
$$

and

$$
d\left(x_{s}-x_{i}\right)=1 \quad \text { if } \quad x_{i}=x_{s}
$$


$d\left(y_{s}-y_{j}\right)$ and $d\left(z_{s}-z_{k}\right)$ are calculated similarly. It is noteworthy to mention that this weighting function is not only used to calculate the IB velocity and force, but also used to determine the surrounding grid points to an IB point when coding. This is because the function only has non-zero values as the distance between the grid point and IB point is within one grid spacing [92].

In three-dimensions, the fluid velocities are interpolated to an IB point $\left(x_{s}, y_{s}, z_{s}\right)$, from the 8 grid points (on the corner of a hexahedral computational cell containing the IB point) denoted by the indices $(i, j, k),(i+1, j, k),(i, j+1, k)$, $(i+1, j+1, k),(i, j, k+1),(i+1, j, k+1),(i, j+1, k+1)$, and $(i+1, j+1, k+1)$.

Since the present study concentrates on the transport of gaseous contaminants (passive scalar) with the flow, IB method is needed to be incorporated into generic passive scalar transport equation (Equation 2.3), as well. Specifically, in this study, penetration of passive scalar into the solid body must be prevented, as physically diffusion of gaseous contaminants into worker's body is not possible. Hence, the diffusion flux, $J$, defined as

$$
J=\Gamma_{e f f}\left(\frac{d \phi}{d n}\right)_{I B}
$$

must be set to zero. Mathematically, this can be achieved by setting either the effective diffusivity, $\Gamma_{e f f}$, or the normal derivative, $\left(\frac{d \phi}{d n}\right)_{I B}$, to zero. The latter approach requires an additional layer of virtual boundary points to deal with the normal derivative. Details of this approach can be found in [92] where a heat transfer equation is solved for temperature. This study, however, employs the former approach as it is more practical and works as intended. 


\subsection{Test Case: Uniform Flow over a Stationary Cylinder}

The present test case is about uniform flow over a stationary circular cylinder at a Reynolds number of 400 , based on the inlet velocity $\left(U_{0}=2 \mathrm{~m} / \mathrm{s}\right)$, diameter of the cylinder $(D=0.4 \mathrm{~m})$ and kinematic viscosity $\left(\nu=2 \times 10^{-3} \mathrm{~m}^{2} / \mathrm{s}\right)$. For this Reynolds number, the flow is in subcritical regime characterized by vortex street instabilities [94]. The aim this test case is to test whether the parallel DREAM with IB method can predict the anticipated flow patterns or not. It will also demonstrate the extent of numerical diffusivity introduced by the QSOU scheme. For these purposes, a computational domain extending from 0 to $20 D$ in the $x$-direction and from 0 to $10 D$ in the $y$-direction is generated. The circular cylinder is located at $(5 D, 5 D)$. The schematic of the computational domain along with the boundary conditions is displayed in Figure 7.3. As one can notice from the figure, a passive scalar, $\phi$, with magnitude of one, is introduced from the bottom half of the inlet $(0<y \leq 5 D)$. The passive scalar at the upper half of the inlet $(5 D<y \leq 10 D)$ is simply zero. The physical diffusivity of the passive scalar is set to zero in order to see the shear layers clearly and to assess the numerical diffusivity. The simulation was started from a stagnant condition and ran for $10 \mathrm{~s}$ with a constant time step of $\Delta t=2 \times 10^{-5} \mathrm{~s}$. The computational grid used is a $400 \times 100$ uniform Cartesian grid. The cylinder is defined by 360 IB points in total. The gains, $\alpha$ and $\beta$, are chosen to be -3000 and -40 , respectively.

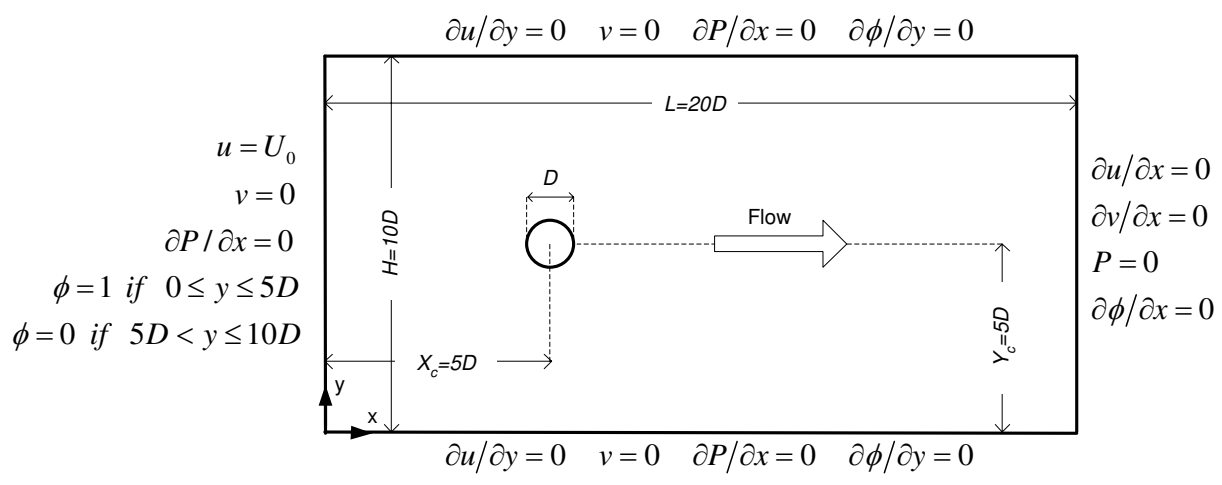

Figure 7.3: Computational domain along with the boundary conditions 
QUICK scheme was used to discretize the convection terms in the momentum equations, whereas QSOU scheme was employed for those appearing in the passive scalar transport equation. Second order central differencing scheme was applied for spatial discretization all of the diffusion terms. For time integration the two-step Adams-Bashforth scheme was used. Computations were performed in parallel using Crank-Nicholson method.

Figures 7.4 and 7.5 demonstrate the instantaneous streamwise velocity and passive scalar fields, respectively. As can be seen from both figures, the characteristic vortex shedding is captured successfully. In Figure, 7.5 the passive scalar with values less then 0.01 is cut off to demonstrate how small numerical diffusion is obtained. The passive scalar seems to track the flow very closely.

Figure 7.6 displays the time history of streamwise velocity at $(6 D, 6.25 D)$. Based on the frequency of the velocity oscillations the calculated Strouhal number is about 0.18 . This value is reasonably close to 0.2 which is reported in the literature [94]. The small discrepancy seen between the values can be attributed to inadequate number of IB points to represent a perfect circular cylinder and/or to the values of $\alpha$ and $\beta$ which may need to be tuned for further for the flow. On the whole, it can be concluded that the DREAM_P code with IB method is capable of predicting unsteady flows. The QSOU scheme seems to produce low numerical diffusion in unsteady flows. Hence, the DREAM_P code is ready to be used for more complex problems. 


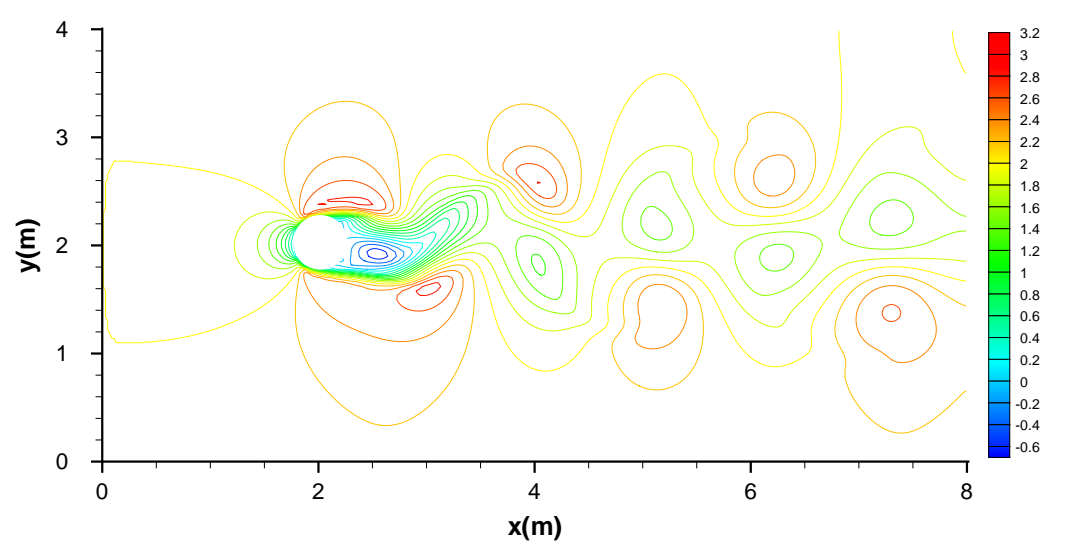

Figure 7.4: Instantaneous streamwise velocity

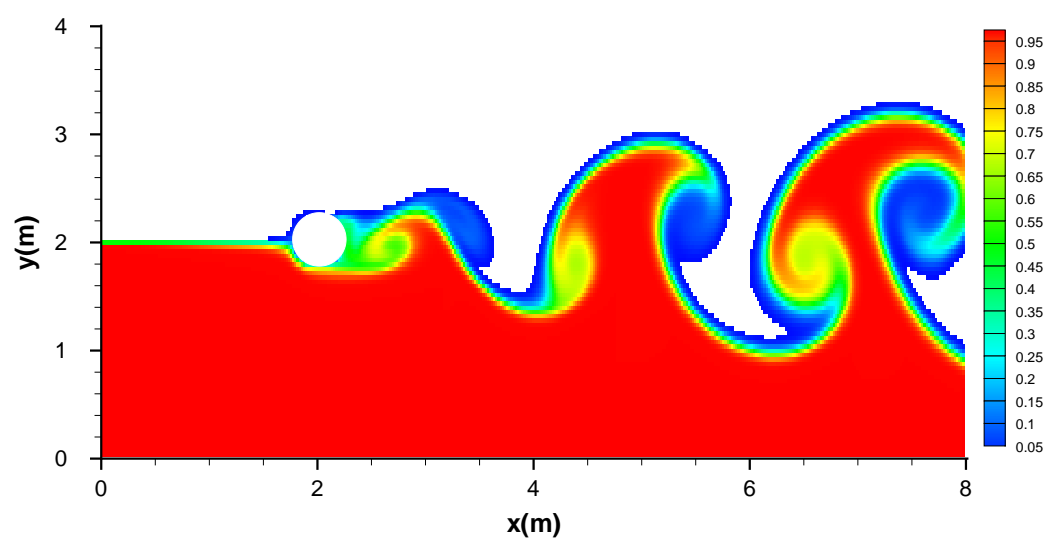

Figure 7.5: Instantaneous passive scalar field, values less than 0.01 is cut off

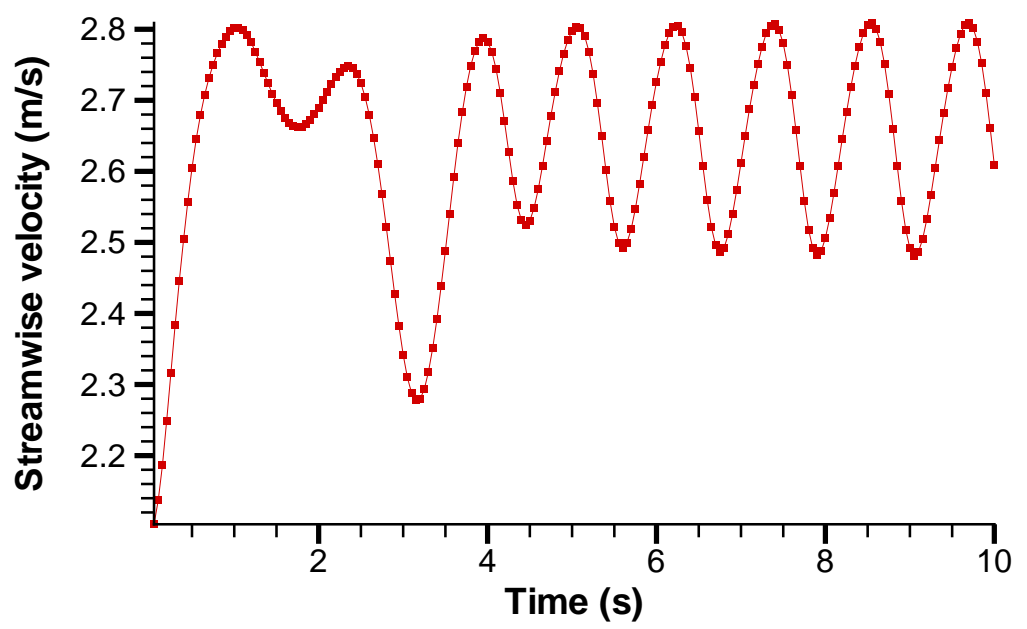

Figure 7.6: Time history of streamwise velocity at (6D, 6.25D) 


\section{Chapter 8}

\section{Application}

Having developed the DREAM code to a stage where LES with IB method can be performed using parallel processing, the focus has now moved to an application of this code to the original enclosing hood / worker configuration introduced in Chapter 4. It is noteworthy to mention that to the author's best knowledge, the IB studies available in the literature mostly concentrate on simpler geometries such as two- and three-dimensional circular or rectangular cylinders, cylinder bundles or spheres. To this end, the geometry considered here (See Figure 8.1) is more complex, despite several simplifications made to the original configuration.

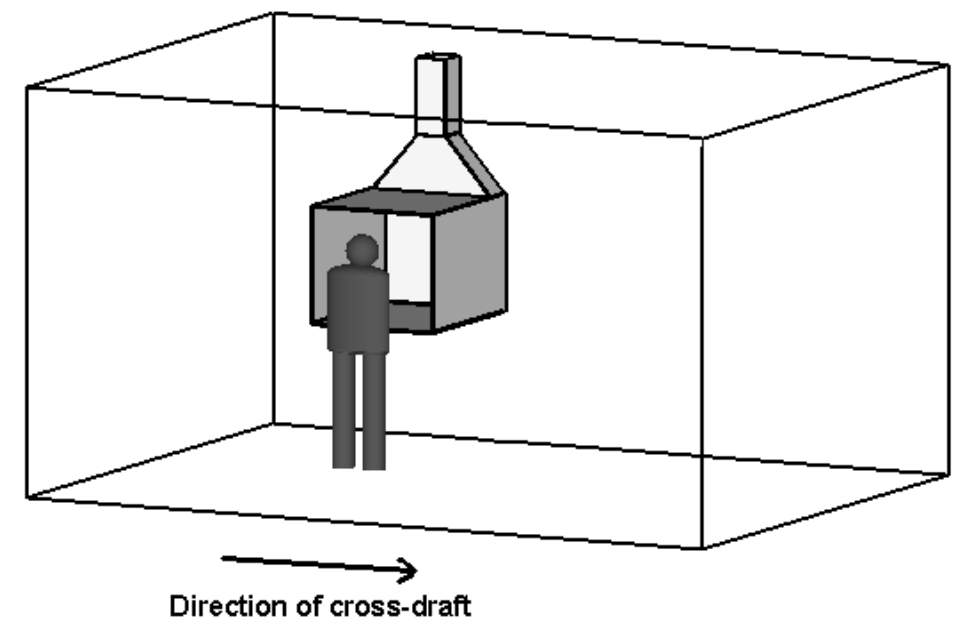

Figure 8.1: Physical representation of the computational domain

The first simplification made is to neglect the rectangular to round transition of the takeoff. Instead, a takeoff with fully rectangular cross-section throughout 
its height was used. Thereby, the exhaust duct connected to the takeoff has a conforming rectangular cross-section instead of a circular one used in the experiments and FLUENT simulations. Second, the plenum section inside the hood was omitted. Third, the contaminant (passive scalar) of magnitude 1.0 was introduced with zero velocity as opposed to the original case where the contaminant was released with a mass flow rate equal to the one used in the experiments. Fourth, the distance between the hood and worker was slightly larger. Also, the body heat was not taken into account; buoyancy effects were neglected.

The simulation was carried out only for Case F ( $V_{\text {Cross-draft }}=0.291 \mathrm{~m} / \mathrm{s}$ and $\left.V_{\text {Face }}=0.969 \mathrm{~m} / \mathrm{s}\right)$ using a uniform grid being $125 \times 68 \times 90$ in the $x-, y-$ and $z$-directions, respectively. The air properties and boundary conditions were set to be identical to those used in the FLUENT simulations presented in Chapter 4. The hood and the worker together were represented with 278,000 IB points in total.

The simulation was started from rest with a time step of $5.0 \times 10^{-5} \mathrm{~s}$. The initial values of $\alpha$ and $\beta$ were -300 and -4 , respectively. After 100 time steps, when the shape of the IB was fully developed, in order to decrease the execution time, the time step was increased to $1.0 \times 10^{-4}$ s while $\alpha$ and $\beta$ were reduced to -10 and -1 , respectively. The simulation was run in parallel on 4 processors using the Crank-Nicholson discretization scheme. For time integration Adams-Bashforth scheme was employed. The convective terms of the momentum equation were discretized using QUICK scheme, whereas the QSOU scheme was applied to discretization of convective terms in the passive scalar transport equation. As for the SGS model, the Smagorinsky model was adopted.

Figure 8.2 shows the time-averaged vector field superimposed on the passive scalar contours on the vertical symmetry plane of the hood. It is clearly seen from the velocity vectors that due to the absence of the plenum section, the effect of suction is felt more profoundly by the air near the hood's top wall as opposed 
the air near the bottom wall (working surface). In that sense, the simulated flow is slightly different than those simulated with FLUENT and observed through flow visualization experiments. Nevertheless, one can see from the figure that the separation of the flow between the worker and hood separates at the front edge of hood's bottom plate and the resulting recirculation zone on the working surface was successfully captured. Conforming with the FLUENT predictions, the upward flow in front of the manikin was found to be responsible for the transport of the contaminants to the worker's breathing zone.

It should be mentioned that no recirculation zone attached to the top hood's wall was captured. This situation might arise from under-resolved boundary layer (coarse grid) as well as the fact that in the absence of the plenum the air in this region is sucked in more strongly compared to the case in FLUENT runs. The positive concentration values outside the hood are within one cell size. This is an expected situation arising from the bi-linear interpolation and also from the interpolation performed by the post-processing tool.

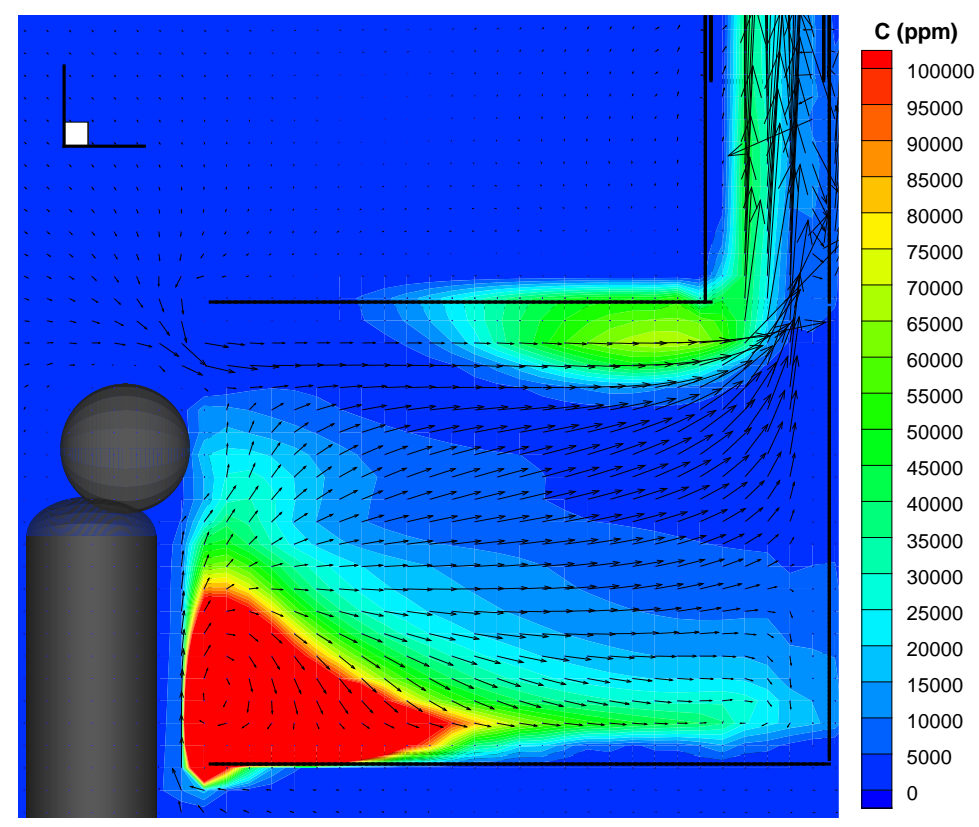

Figure 8.2: Flow and concentration fields on the vertical symmetry plane of the hood

Figure 8.3 displays the flow and concentration fields at the hood face and further downstream. From the figure DREAM_P does appear to have the ability 
predict the vortical motions in front of the worker and right above the working place. Similar to FLUENT predictions, a great deal of contaminants are found to be trapped in the recirculation zone attached to the left wall of the hood. This is more clearly seen in Figure 8.4 where the concentration and flow distribution at the level of belly and head are shown. Unlike the case with the plenum section, the recirculation zones are extending to the back wall of the hood and also the recirculation zone attached to the right wall is significantly larger. These are the effects brought about by the absence of the plenum.

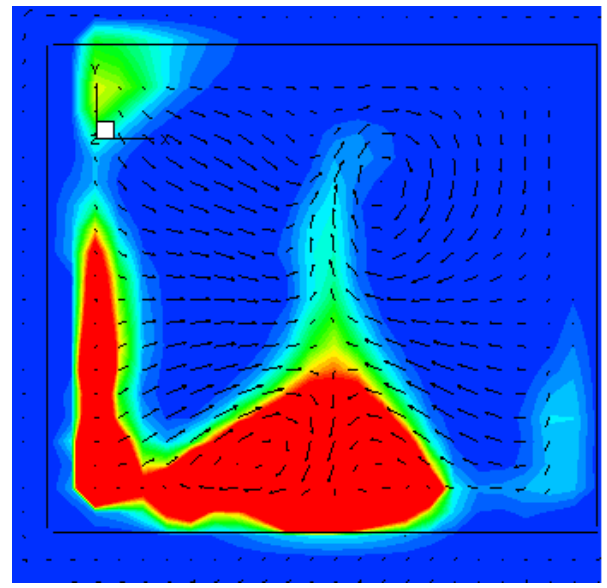

a)

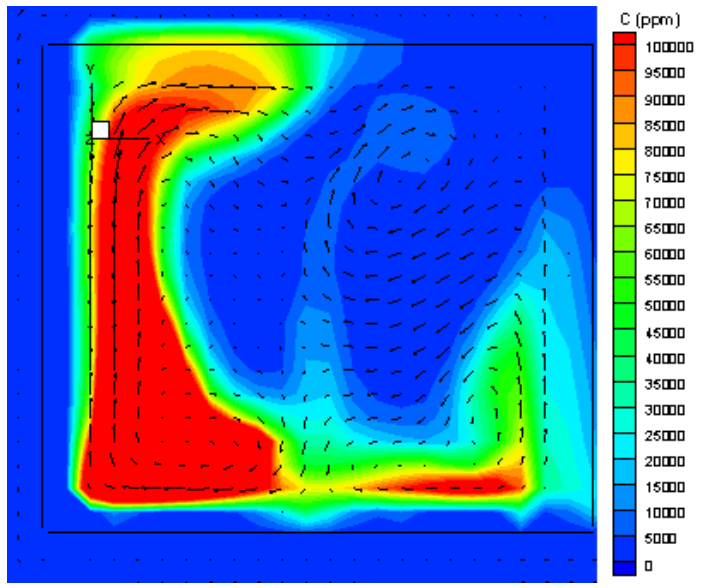

b)

Figure 8.3: Instantaneous flow and concentration fields on the $x y$-planes a) $z=2.06 \mathrm{~m}$ (hood face), b) $z=2.41 \mathrm{~m}$

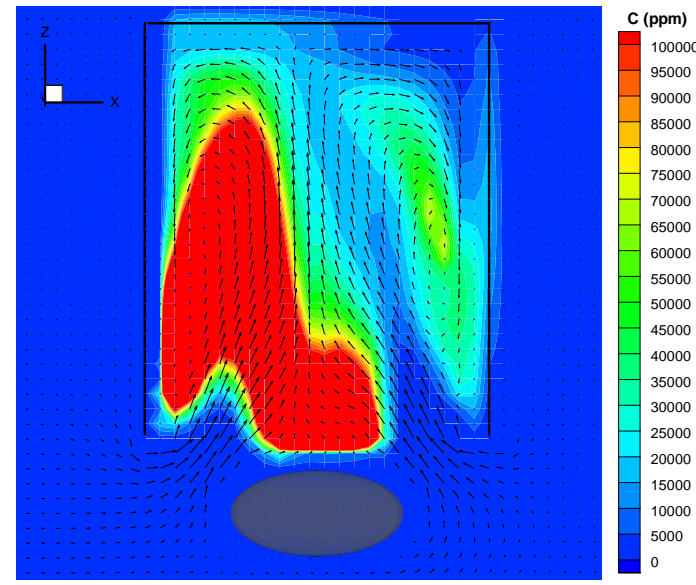

a)

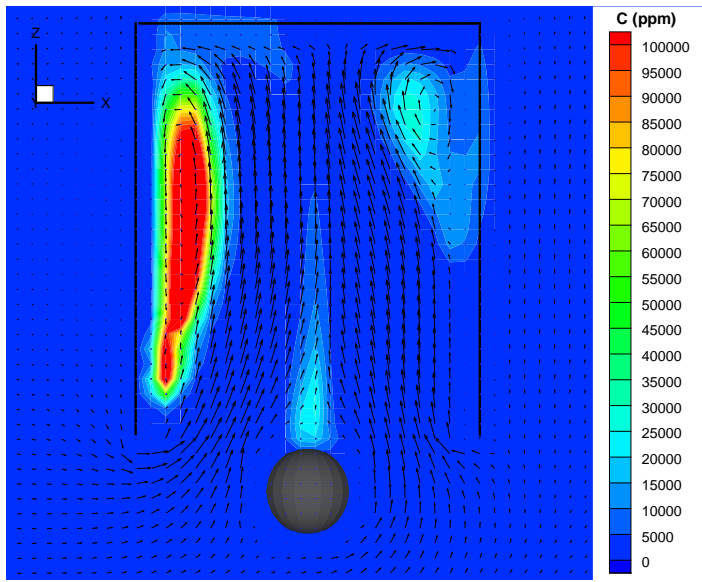

b)

Figure 8.4: Instantaneous flow and concentration fields on the $x z$-planes a) $y=1.19 \mathrm{~m}, \mathrm{~b}) z=1.47 \mathrm{~m}$ 
Originally, more details about the flow were expected to be revealed with the LES, however, due to the under-resolved boundary layer around the worker, most of the anticipated boundary layer physics could not be captured. This is also apparent from the time history of $z$-velocity at a monitoring point in front of the worker's head (see Figure 8.5). Clearly, the flow is unsteady there, however, considering the high suction rate, the high frequency oscillations seen in FLUENT predictions (See Figure 4.30b), could not be captured. The frequency associated with large scale oscillation is about $0.1 \mathrm{~Hz}$ leading to a Strouhal number of 0.07. This value compares reasonably well with the Strouhal number predicted by the FLUENT (0.11).

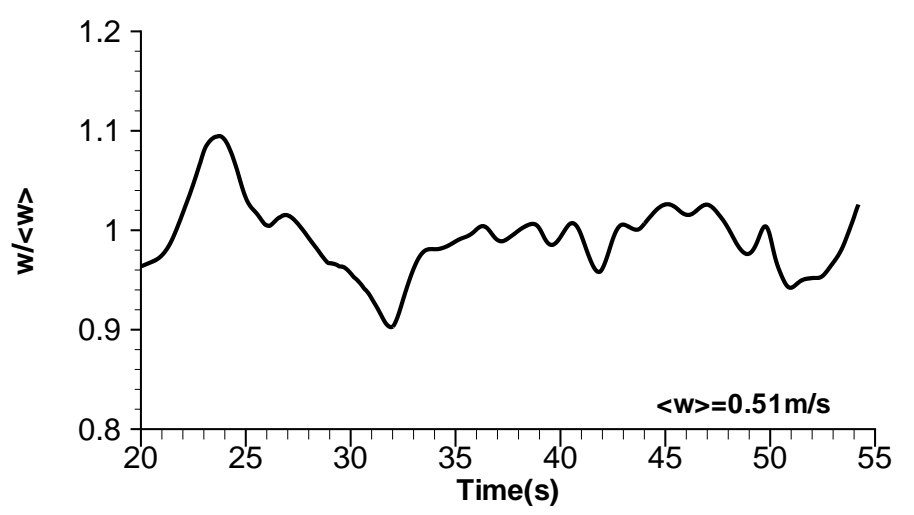

Figure 8.5: Time history of $z$-velocity at a point in the wake of the worker $(x=1.96 \mathrm{~m}, y=1.43 \mathrm{~m}, z=1.92 \mathrm{~m})$

In an attempt to test the performance of the DREAM_P code, another simulation was run with FLUENT using about the same total number of computational cells $(764,853)$. The same discretization schemes and approximately the same convergence criteria were set. The simulations were run for several time step to obtain the average wall-clock time per iteration. Comparisons show that the DREAM_P (0.16s per iteration) is about 24 times faster than FLUENT (3.8s per iteration). This significantly higher performance achieved by the DREAM_P code is possibly due in part to the using IB method instead of body-fitted grid approach and due in part to the non-iterative projection method that DREAM_P for velocity-pressure coupling method. FLUENT uses iterative methods that slow down the execution speed. In the 
present FLUENT simulations, SIMPLEC method was chosen as the velocitypressure coupling method to be consistent with other runs from which the results are presented in the preceding chapters.

On the whole, even though some of the anticipated physics could not be captured due to inadequate grid resolution, the results are encouraging with regard to predicting large scale motion. It seems that with further modifications that can enable using higher degree of grid resolutions, localized phenomena occurring in the worker's wake can be captured better and more efficiently compared to FLUENT. Finally, from the viewpoint of applicability of DREAM_P to other bluff body dominated flows, it is only a matter of defining the geometry of the solid body. This can open up opportunities to import a CAD model of any solid body into DREAM_P and simulate the flow around it efficiently. 


\section{Chapter 9}

\section{Conclusions and Future Recommendations}

\subsection{Conclusions}

Effects of physical factors on the flow dynamics and associated contaminant transport taking place in the wake of a worker standing in front of a bench-top enclosing hood were numerically investigated. The physical factors considered include relative strength of cross-draft to suction force created by the hood fan, and the worker's body heat and shape. In addition, effects of numerical factors, such as grid structure, grid size and turbulence models on the accuracy of the simulations were scrutinized. Flow visualization experiments were performed using smoke tests in order to obtain qualitative information about the airflow. The numerical results were compared to available experimental data for exposure trend analysis.

Based on both numerical and experimental analyses, the effect of cross-draft relative to the suction was found to exhibit a general trend where the level of exposure increases with increasing $V_{\text {Cross-draft }}$ to $V_{\text {Face }}$ ratio until a critical value; then with further increase in $V_{\text {Cross-draft }}$ to $V_{\text {Face }}$ ratio, the exposure level starts to decline. In general, trends of the numerical and the experimental results agree well with each other. The grid convergence study revealed that the quantitative numerical results are highly sensitive to the grid resolution. It is difficult to obtain grid independent results for the cases investigated. Hence, only the trends rather than quantitative values were the focus. 
The thermal impact of the worker's body was found to be more pronounced at low values of $V_{\text {Cross-draft }}$ to $V_{\text {Face }}$ ratio. Even so, the buoyancy effects were found capable of only perturbing the wake flow rather than totally changing the flow pattern. Nevertheless, small perturbations to the airflow seen at low flowrates led to remarkably negative influence on the exposure level. Therefore, without including the buoyancy effects, results from isothermal simulations of similar flows may be misleading.

From the viewpoint of flow structures in the wake of the worker, vortical motions extending towards to back of the hood were observed. The combined effect of cross-draft and suction, however, seem to prevent formation of anticipated recirculation zone in the wake of the worker's torso. Nonetheless, an upward flow in front of the worker and a large recirculation zone right above the working surface were captured. In fact, these flow patterns were also detected through flow visualization. When the draft comes from the worker's back, seemingly large-scale lateral vortices were predicted. This pointed out that this particular configuration is a more severe case in that the resulting vortices can draw contaminants elsewhere in the hood and can lead to a higher exposure level overall.

Analyses showed that the vortical motions in the near-wake of the worker are unsteady. More precisely, both the location and the size of the vortices change with time. This indicates that in uncontrolled environments many other factors and their interaction may exacerbate this behavior of the flow which, in turn, can augment the variability of exposure levels. To this end, controlling or minimizing the disturbing extraneous factors seems like as important as designing effective hoods. Otherwise, large variability in exposure levels would be expected.

Comparison of the numerical results obtained with simple, round body and anthropometrically-scaled, complex body revealed that the simple round body is an acceptable representation of the realistic body from the viewpoint of exposure level. The stationary arms of the anthropometrically-scaled complex manikin 
which extend into the hood seem to have negligible influence on the transport of contaminants to the breathing zone. However, in reality, worker activity is one of the most important factors that may affect the level of exposure.

To study the role of turbulence models, a two-dimensional test case, originally proposed by Dunnett [4] was revisited within the context of testing several RANS turbulence models. The predictions suggested that the SST $k-\omega$ model is more responsive to unsteady flow dynamics. The RNG $k-\epsilon$ and Standard $k-\epsilon$ models, on the other hand, are found overly diffusive, and hence, are not as successful as the SST $k-\omega$ model in capturing the unsteady phenomena. With adequate grid resolution around the solid body, lateral recirculation zones were captured. These recirculation zones were not captured in the original study by Dunnett [4].

In a further attempt to test the effect of RANS models on the predicted physics, a simulation was run without any turbulence model and richer flow physics was captured. This suggest that LES might suit better for this kind of flows. To this end, DREAM was modified significantly to serve the purpose. The new code (DREAM_P) was then applied for LES of the original worker-hood problem. The results agree favorably with the URANS simulations. On the whole the DREAM_P forms a much simpler computational platform compared to FLUENT for further study of flows dominated by bluff bodies.

\subsection{Future Recommendations}

Based on the experience gained from the current numerical study, the following recommendations for future extension of the work are suggested.

- Additional simulations could be performed on finer meshes to draw a stronger conclusion about the sensitivity of the results to the grid resolution.

- The effect of random disturbances to the flow representing out-of-hand changes that occur during the experiments could be investigated. 
- Modifications can be introduced to the IB method with regard to relaxing CFL number restriction in order to capture the flow physics better. This could be achieved by applying implicit forcing instead of explicit forcing, or by applying logarithmic time transformation to reduce the stiffness of the momentum equations

- Movements of worker's arms can be incorporated in the simulation in order to take worker's activity into account. With the IB method, this is can be achieved easily.

- The DREAM_P was coded such that the IB is contained in a single subdomain. In other words, it can not span across multiple sub-domains. This limits the execution speed because the sub-domain holding the IB has to have the adequate (densest) grid resolution. Enabling the IB span across multiple sub-domains would alleviate this is critical limitation in the current version of DREAM_P 


\section{Bibliography}

[1] W. Burgess, M. Ellenbecker, and R. Treitman, Ventilation for Control of the Work Environment. John Wiley and Sons, Inc., Hoboken, New Jersey, USA, 2004.

[2] D. Hughes, A Literature Survey and Design Study of Fume Cupboards and Fume Dispersal Systems. Occupational Hygiene Monograph No. 5. ed D. Hughes. H and H Scientific Consultants Ltd. in association with Science Reviews Ltd., Northwood, Middlesex, UK, 1980.

[3] National Research Council, Prudent Practices in the Laboratory: Handling and Disposal of Chemicals. National Academy Press, Washington, DC, USA, 1995, p. 179.

[4] S. Dunnett, "A numerical investigation into the flow field around a worker positioned by an exhaust opening," Ann Occup Hyg, vol. 38, no. 5, pp. 663 - 686, 1994.

[5] D. Ingham and Y. Yuan, "A mathematical model for the air flow around a worker near a fume cupboard," Ann Occup Hyg, vol. 36, no. 5, pp. $441-$ 453, 1992.

[6] N. Delany and N. Sorensen, "Low-speed drag of cylinders of various shapes," National Advisory Committee on Aeronautics, Washington, DC, Tech. Rep. NACA TN-3038, 1953. 
[7] B. Ljungqvist, "Air movements - the dispersion of pollutants. studies with illustrative methods," ASHRAE Trans, vol. 93, pp. 1304 - 1317, 1987.

[8] B. Ljungqvist, "Aerodynamic design of fume cupboards," Safety and Hlth Pract, vol. 9, pp. 36 - 40, 1991.

[9] B. Altemose, M. Flynn, and J. Sprankle, "Application of a tracer gas challenge with a human subject to investigate factors affecting the performance of laboratory fume hoods," Am Ind Hyg Assoc J, vol. 59, no. 5, pp. $321-327,1998$.

[10] L.-C.Tseng, R. Huang, C.-C. Chen, and C.-P. Chang, "Correlation between airflow patterns and performance of a laboratory fume hood," J Occup Environ Hyg, vol. 3, no. 12, pp. 694 - 706, 2006.

[11] R. Huang, Y. Wu, H. Chen, C.-C. Chen, C.-W. Chen, C.-P. Chang, and T.S. Shih, "Development and evaluation of an air-curtain fume cabinet with considerations of its aerodynamics," Ann Occup Hyg, vol. 51, no. 2, pp. 189 $-206,2006$.

[12] J. Li, I. Yavuz, I. Celik, and S. Guffey, "A numerical study of worker exposure to a gaseous contaminant: Variations on body shape and scalar transport model," J Occup Environ Hyg, vol. 2, no. 6, pp. 323 - 334, 2005.

[13] MARNET-CFD Best Practice Guidelines for Marine Applications of CFD, [Online]. Available: https://pronet.wsatkins.co.uk/marnet/ publications/bpg.pdf , last visited on: 1/20/2011.

[14] Z. Zhai, Z. Zhang, W. Zhang, and Q. Chen, "Evaluation of various 
turbulence models in predicting airflow and turbulence in enclosed environments by CFD: Part 1 summary of prevalent turbulence models," HVAC Res. Journal, vol. 13, no. 6, pp. 853 - 870, 2007.

[15] S. Pope, Turbulent Flows. Cambridge University Press; 5th edition, New York, USA, 2008.

[16] What are artificial numerical viscosities?, [Online]. Available: http://www.flow3d.com/cfd-101/cfd-101artificial-viscosities.html, last visited on: 12/7/2010

[17] I. Celik, Z. Cehreli, and I. Yavuz, "Index of resolution quality for large eddy simulation," J Fluid Eng, vol. 127, pp. 949 - 958, 2005.

[18] N. Lan and S. Viswanathan, "Numerical simulation of airflow around a variable volume/constant face velocity fume cupboard," Am Ind Hyg Assoc J, vol. 62, no. 3, pp. $303-312,2001$.

[19] P. Hu, D. Ingham, and X. Wen, "Effect of the location of the exhaust duct, an exterior obstruction and handle on the air flow inside and around a fume cupboard," Ann Occup Hyg, vol. 40, no. 2, pp. 127 - 144, 1996.

[20] G. Nicholson, R. Clark, and M. de Calcina-Goff, "Computational fluid dynamics as a method for assessing fume cupboard performance," Ann Occup Hyg, vol. 44, no. 3, pp. 203 - 217, 2000.

[21] M. Chern and W. Cheng, "Numerical investigation of turbulent diffusion in push-pull and exhaust fume cupboards," Ann Occup Hyg, vol. 51, no. 6, pp. $517-31,2007$. 
[22] B. Craven and G. Settles, "A computational and experimental investigation of the human thermal plume," J Fluid Eng, vol. 128, no. 6, pp. 1251 - 1258, 2006.

[23] H. Gowadia and G. Settles, "The natural sampling of airborne trace signals from explosives concealed upon the human body," J Forensic Sci, vol. 46, no. 6, pp. $1324-1331,2001$.

[24] R. P. Clark and E. C. Edholm, Man and His Thermal Environment. E. Arnold Ltd., London, UK 1985.

[25] S. Murakami, S. Kato, and J. Zeng, "Combined simulation of airflow, radiation and moisture transport for heat release from a human body," Build Environ, vol. 35, no. 6, pp. 489 - 500, 2000.

[26] S. Murakami, "Analysis and design of micro-climate around the human body with respiration by CFD," Indoor Air, vol. 14, no. s7, pp. $144-156$, 2004.

[27] H. E. Lewis, A. R. Foster, B. J. Mullan, R. N. Cox, and R. P. Clark, "Aerodynamics of the human microenvironment," The Lancet, vol. 293, no. 7609, pp. $1273-1277,1969$.

[28] D. Heist, A. Eisner, W. Mitchell, and R. Wiener, "Airflow around a childsize manikin in a low-speed wind environment," Aerosol Sci. Technol., vol. 37, no. 4, pp. $303-314,2003$.

[29] A. Johnson, B. Fletcher, and C. Saunders, "Air movement around a worker in a low-speed flow field," Ann Occup Hyg, vol. 40, no. 1, pp. 57 - 64, 1996. 
[30] S. Murakami, J. Zeng, and T. Hayashi, "CFD analysis of wind environment around a human body," J Wind Eng Ind Aerod, vol. 83, pp. 393 - 408, 1999.

[31] R. Bird, W. Stewart, and E. Lightfoot, Transport Phenomena. John Wiley and Sons, Inc., USA, 2002.

[32] J. Hinze, Turbulence. McGraw-Hill Publishing Co., New York, USA, 1975.

[33] B. Launder and D. Spalding, "The numerical computation of turbulent flows," Comput Method Appl M, vol. 3, no. 2, pp. 269 - 289, 1974.

[34] Fluent 6.3 User's Guide.

[35] S. H. Lam, "On the RNG theory of turbulence," Phys. Fluids A, vol. 4, pp. $1007-1017,1992$.

[36] F. Menter, "Two-equation eddy-viscosity turbulence models for engineering applications," AIAA Journal, vol. 38, no. 2, pp. 1598 - 1605, 1994.

[37] D. Wilcox, Turbulence Modeling for CFD. DCW Industries, Inc., La Canada, California, 1998.

[38] FIDAP Update Manual. Fluid Dynamics Analysis Package; 1995. pp. 3 13.

[39] T. Cebeci, Analysis of Turbulent Flows. Elsevier Science; 2nd edition, 2004, pp. 204.

[40] N. Mulvany, L. Chen, J. Tu, and B. Anderson, "Steady-state evaluation of 
'two-equation' RANS (reynolds-averaged navier-stokes) turbulence models for high-reynolds number hydrodynamic flow simulations," Australian Government, Department of Defence, Maritime Platforms Division (MPD), Platform Sciences Laboratory, Washington, DC, Tech. Rep. DSTO-TR1564, 2004.

[41] J. Smagorinsky, "General circulation experiments with the primitive equations," Mon Weather Rev, vol. 91, no. 3, pp. 99 - 164, 1963.

[42] D. Lilly, "On the application of the eddy viscosity concept in the inertial subrange of turbulence," in NCAR Manuscript 123., 1966.

[43] P. Voke, "Subgrid-scale modelling at low mesh Reynolds number," Theor Comp Fluid Dyn, vol. 8, pp. 131 - 143, 1996.

[44] C. Meneveau and T. Lund, "The dynamic Smagorinsky model and scaledependent coefficients in the viscous range of turbulence," Phys Fluids, vol. 9, no. 12, pp. $3932-3934,1997$.

[45] M. Germano, U. Piomelli, P. Moin, and W. Cabot, "A dynamic subgridscale eddy viscosity model," Phys Fluids A: Fluid Dynamics, vol. 3, no. 7, pp. $1760-1765,1991$.

[46] D. Lilly, "A proposed modification of the Germano subgrid-scale closure method," Phys Fluids A: Fluid Dynamics, vol. 4, no. 3, pp. 633 - 635, 1992.

[47] C. Meneveau, T. Lund, and W. Cabot, "A Lagrangian dynamic subgridscale model of turbulence," J Fluid Mech, vol. 319, pp. 353 - 385, 1996. 
[48] C. Meneveau and J. Katz, "Scale-invariance and turbulence models for large-eddy simulation," Annu Rev Fluid Mech, vol. 32, no. 1, pp. 1 - 32, 2000.

[49] G. Hu, "Towards large eddy simulation of dispersed gas-liquid twophase turbulent flows," Ph.D. dissertation, West Virginia University, Morgantown, WV, USA, 2005.

[50] D. George, M. Flynn, and R. Goodman, "The impact of boundary layer separation on local exhaust design and worker exposure," Appl Occup Environ Hyg, vol. 5, pp. 501 - 509, 1990.

[51] R. Franke and W. Rodi, "Calculation of vortex shedding past a square cylinder with various turbulence models," in Turbulent Shear Flows 8, Tech. Univ Munich, Springer Berlin, 1991, pp. 189 - 204.

[52] S. Guffey, 2010, private communication.

[53] X. He, "Effects of face velocity, cross-draft velocity and interventions on the performance of a benchtop enclosing hood," Master's thesis, West Virginia University, Morgantown, WV, USA, 2010.

[54] V. Yakhot, S. Thangam, T. Gatski, S. Orszag, and C. Speziale, "Development of turbulence models for shear flows by a double expansion technique," Phys Fluids A, vol. 4, no. 7, pp. 1510 - 1520, 1998.

[55] H. Kim and P. Durbin, "Observations of the frequencies in a sphere wake and of drag increase by acoustic excitation," Phys Fluids, vol. 31, no. 11, pp. 3260 - 3265, 1988. 
[56] M. Snir, S. Otto, S. Huss-Lederman, D. Walker, and J. Dongarra, MPI: The Complete Reference. MIT Press Cambridge, MA, USA, 1995.

[57] H. Pan and M. Damodaran, "Parallel computation of viscous incompressible flows using godunov-projection method on overlapping grids," Int J Numer Meth Fl, vol. 39, no. 5, pp. $441-463,2002$.

[58] U. Ghia, K. Ghia, and C. Shin, "High-Re solutions for incompressible flow using the Navier-Stokes equations and a multigrid method," J Comput Phys, vol. 48, no. 3, pp. 387 - 411, 1982.

[59] L. Carvalho and J. Palma, "Parallelization of CFD code using PVM and domain decomposition techniques," in Vector and Parallel Processing VECPAR'96, ser. Lecture Notes in Computer Science, J. Palma and J. Dongarra, Eds. Springer Berlin / Heidelberg, 1997, vol. 1215, pp. 247 257.

[60] C. Ersahin, T. Tarhan, I. Tuncer, and N. Selcuk, "Parallelization of a transient method of lines Navier-Stokes code," Int J Comput Fluid Dyn, vol. 18 , no. 1 , pp. $81-92,2004$.

[61] A. Camargos, R. Batalha, C. Martins, E. Silva, and G. Soares, "Superlinear speedup in a 3-d parallel conjugate gradient solver," IEEE Transactions on Magnetics, vol. 45, no. 3, pp. 1602 - 1605, 2009.

[62] D. Lyn, S. Einav, W. Rodi, and J.-H. Park, "A laser-doppler velocimetry study of ensemble-averaged characteristics of the turbulent near wake of a square cylinder," J Fluid Mech, vol. 304, pp. 285 - 319, 1995. 
[63] W. Rodi, "Comparison of les and RANS calculations of the flow around bluff bodies," J Wind Eng Ind Aerod, vol. 69-71, pp. 55 - 75, 1997, proceedings of the 3rd International Colloqium on Bluff Body Aerodynamics and Applications.

[64] W. Rodi, J. Ferziger, M. Breuer, and M. Pourquie, in Workshop on LargeEddy Simulation of Flows Past Bluff Bodies, Rottach-Egern, Germany, 1995.

[65] W. Rodi, J. Ferziger, M. Breuer, and M. Pourquiee, "Status of large eddy simulation: Results of a workshop," J Fluid Eng, vol. 119, no. 2, pp. 248 $262,1997$.

[66] W. Rodi, "Large-eddy simulations of the flow past bluff bodies : Stateof-the-art," JSME international journal. Ser. B, Fluids and thermal engineering, vol. 41, no. 2, pp. $361-374,1998$.

[67] J. Chollet, P. Voke, and L. K. eds., "Flow past a square cylinder: Test case LES2," in Direct and Large-Eddy Simulation II, Kluwer Acedemic, Dordrecht, 1997.

[68] KIVA-II: A Computer Program for Chemically Reactive Flows with Sprays, Los Alamos National Laboratory, LA-11560-MS, 1989.

[69] K. Richards, E. Pomraning, P. Senecal, C. Choi, and C. Rutland, "On the use of the immersed boundary method for engine modeling," International Multidimensional Engine Modeling Users Group (Meeting), Meeting Report, March 2003. 
[70] D. Goldstein, R. Handler, and L. Sirovich, "Modeling a no-slip flow boundary with an external force field," J Comput Phys, vol. 105, no. 2, pp. $354-366,1993$.

[71] C. Peskin, "Flow patterns around heart valves: A numerical method," $J$ Comput Phys, vol. 10, no. 2, pp. 252 - 271, 1972.

[72] C. Peskin, "Numerical analysis of blood flow in the heart," J Comput Phys, vol. 25, no. 3, pp. 220 - 252, 1977.

[73] A. Fogelson, "Continuum models of platelet aggregation: Formulation and mechanical properties," SIAM J Appl Math, vol. 52, no. 4, pp. 1089 - 1110, 1992.

[74] R. Dillon, L. Fauci, and I. D. Gaver, "A microscale model of bacterial swimming, chemotaxis and substrate transport," J Theor Biol, vol. 177, no. 4 , pp. $325-340,1995$.

[75] N. Wang and A. Fogelson, "Computational methods for continuum models of platelet aggregation," J Comput Phys, vol. 151, no. 2, pp. 649 - 675, 1999.

[76] M.-C. Lai and C. Peskin, "An immersed boundary method with formal second-order accuracy and reduced numerical viscosity," J Comput Phys, vol. 160, no. 2, pp. $705-719,2000$.

[77] E. M. Saiki and S. Biringen, "Numerical simulation of a cylinder in uniform flow: Application of a virtual boundary method," J Comput Phys, vol. 123, no. 2, pp. $450-465,1996$. 
[78] D. Lee, M. Ha, S. Kim, and H. Yoon, "Application of immersed boundary method for flow over stationary and oscillating cylinders," J Mech Sci Technol, vol. 20, pp. 849 - 863, 2006.

[79] S. Su, M. Lai, and C. Lin, "An immersed boundary technique for simulating complex flows with rigid boundary," Comput and Fluids, vol. 36, no. 2, pp. $313-324,2007$.

[80] E. A. Fadlun, R. Verzicco, P. Orlandi, and J. Mohd-Yusof, "Combined immmersed-boundary finite-difference methods for three-dimensional complex flow simulations," J Comput Phys, vol. 161, no. 1, pp. 35 - 60, 2000.

[81] D. Goldstein and T. Tuan, "Secondary flow induced by riblets," J Fluid Mech, vol. 363, pp. 115 - 151, 1998.

[82] K. Arthurs, L. Moore, C. Peskin, E. Pitman, and H. Layton, "Modeling arteriolar flow and mass transport using the immersed boundary method," J Comput Phys, vol. 147, no. 2, pp. 402 - 440, 1998.

[83] D. Le, B. Khoo, and K. Lim, "An implicit-forcing immersed boundary method for simulating viscous flows in irregular domains," Comput Method Appl M, vol. 197, no. 25-28, pp. 2119 - 2130, 2008.

[84] J. Mohd-Yusof, "Combined immersed-boundary/b-spline methods for simulations of flow in complex geometries," Center for Turbulence Research, NASA Ames/Stanford Univ., Tech. Rep., 1997.

[85] R. Verzicco, J. Mohd-Yusof, P. Orlandi, and D. Haworth, "Large eddy 
simulation in complex geometric configurations using boundary body forces," AIAA Journal, vol. 38, no. 3, pp. 427 - 433, 2000.

[86] R. Mittal and G. Iaccarino, "Immersed boundary methods," Annu Rev Fluid Mech, vol. 37, no. 1, pp. 239 - 261, 2005.

[87] H. Dong, R. Mittal, and F. Najjar, "Wake topology and hydrodynamic performance of low-aspect-ratio flapping foils," J Fluid Mech, vol. 566, pp. $309-343,2006$.

[88] J. Ravoux, A. Nadim, and H. Haj-Hariri, "An embedding method for bluff body flows: Interactions of two side-by-side cylinder wakes," Theor Comput Fluid Dynam, vol. 16, pp. 433 - 466, 2003.

[89] A. Lima, E. Silva, A. Silveira-Neto, and J. Damasceno, "Numerical simulation of two-dimensional flows over a circular cylinder using the immersed boundary method," J Comput Phys, vol. 189, no. 2, pp. 351 $-370,2003$.

[90] Y. Tseng and J. Ferziger, "A ghost-cell immersed boundary method for flow in complex geometry," J Comput Phys, vol. 192, no. 2, pp. 593 - 623, 2003.

[91] N. Zhang and Z. Zheng, "An improved direct-forcing immersed-boundary method for finite difference applications," J Comput Phys, vol. 221, no. 1, pp. $250-268,2007$.

[92] N. Zhang, Z. Zheng, and S. Eckels, "Study of heat-transfer on the surface of a circular cylinder in flow using an immersed-boundary method," Int $J$ Heat Fluid Fl, vol. 29, no. 6, pp. 1558 - 1566, 2008. 
[93] J. R.P. Beyer, "A computational model of the cochlea using the immersed boundary method," J Comput Phys, vol. 98, no. 1, pp. 145 - 162, 1992.

[94] H. Schlichting and K. Gersten, Boundary-Layer Theory. Springer; 8th edition, Berlin, Germany, 2008, p. 22.

[95] T. Varady, R. Martin, and J. Cox, "Reverse engineering of geometric models-an introduction," Comput Aided Design, vol. 29, no. 4, pp. 255 - 268, 1997. 


\section{APPENDIX A}

\section{CAD Modeling of Anthropometrically-scaled Complex Manikin}

Digitizing three-dimensional objects to obtain a geometrically identical model is one of the reverse engineering modeling techniques. In this work a reverse engineering technique called rapid surfacing based on polygonal mesh was adopted to obtain a CAD model of the manikin, and a CAD software called 3DReshaper was used to perform the necessary operations. A typical rapid surfacing process undergoes three phases; point phase, polygon phase and surface phase.

In point phase, the geometric model of an object is recovered by generating a three-dimensional digitized position data as a cloud of points. Coordinate measuring machines (CMMs), digital photogrammetry systems and 3D laser scanners are the three main techniques [95] used for digitization of 3D objects. Among these techniques, 3D laser scanner is the broadest and most popular one due to its relatively fast acquisition rates and robustness. In the present work the manikin was scanned with a three-dimensional laser scanner. Figure A.1 illustrates the point cloud obtained from a laser-scan of the manikin. The next step is to edit the point could for reduction of density in selected regions, and to remove the noises. Fortunately, the point cloud of the manikin did not have much noise except some outliners, some of which are shown in Figure A.2. Once the point cloud is cleaned, the data can be wrapped into a triangle mesh and 


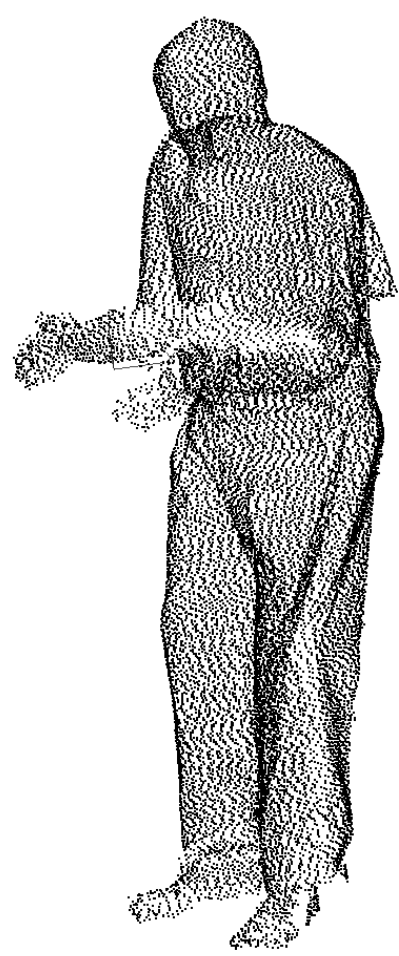

Figure A.1: Unorganized point cloud obtained from 3D laser scanner

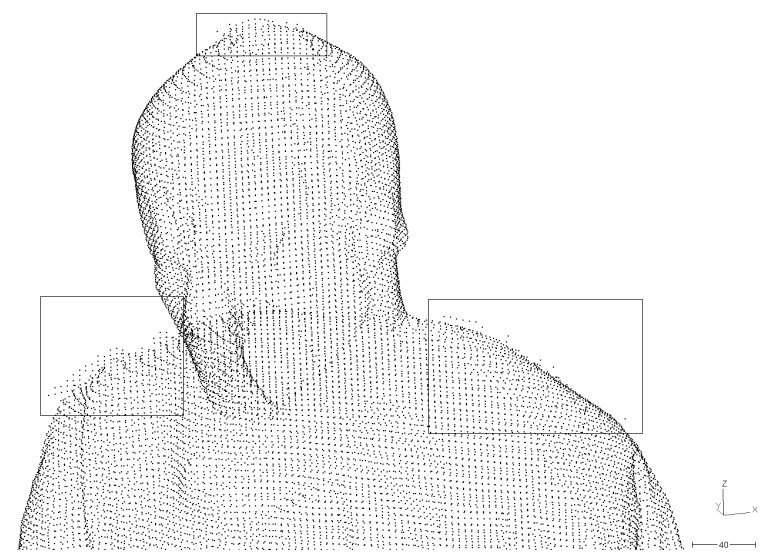

Figure A.2: Outlier points around the head and shoulder

moved to the polygon phase. In the polygon phase, the triangle model can be smoothed and holes can be filled. Although the point cloud did not have much noise, it had considerable amount of holes due to wrinkles of cloths and position of the arms. A big hole around at the waist and arms is shown in Figure A.3. In hole filling operation gaps in the point clouds are filled by inserting new points 


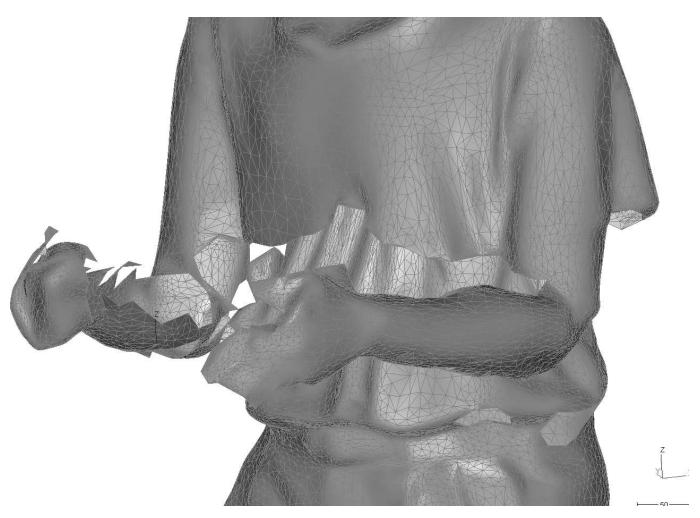

Figure A.3: A big hole on the surface

taking into account the curvature and density of the surrounding points.

Figure A.4 shows the head and shoulders of the manikin after removal of outliners and introducing new points to fill the holes. Figure A.5 shows the

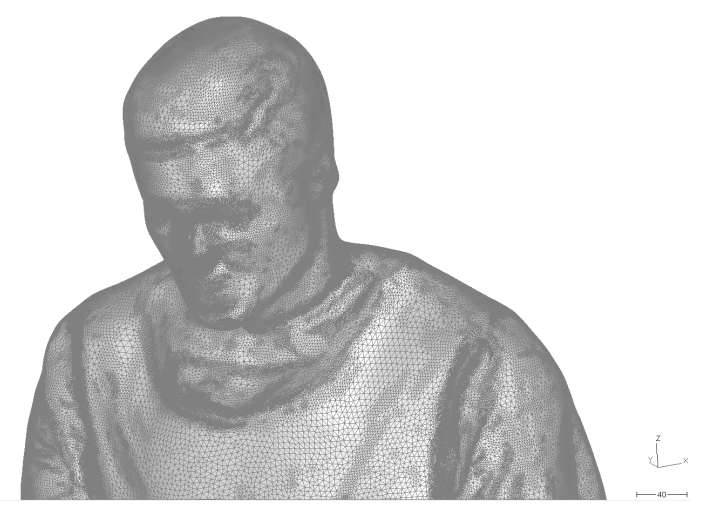

Figure A.4: Fixed head and shoulders

whole body after healing and triangulation. The air-tight model is composed of $2,732,506$ triangles forming a faceted geometry. In this form, it is not possible to use this model in simulations, due to its extremely high and redundant memory requirement. In the surface phase, faceted geometry is converted to a solid model by patching Non-Uniform Rational B-Spline (NURBS) surfaces. The final form of the model is shown in Figure A.6. The lines on the manikin's body are seams for NURBS surfaces. 


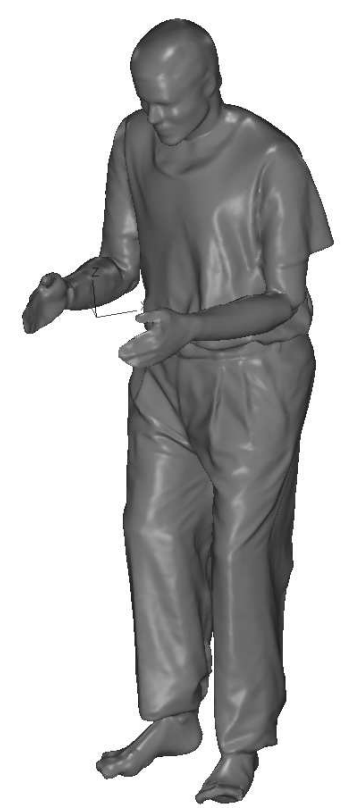

Figure A.5: Healed faceted geometry

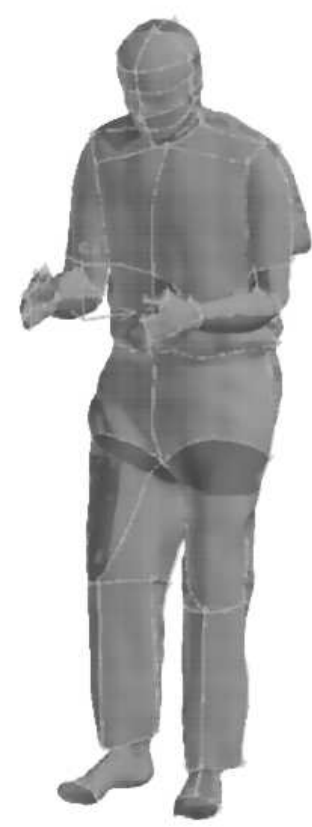

Figure A.6: NURBS surfaces patched onto faceted geometry

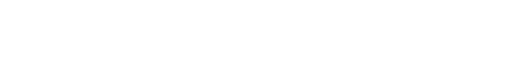

$\mathrm{DN}$ : $\mathrm{Cn}=J$ John H. Hagen, $\mathrm{o}=W$
Virginia University Libraries, 